\title{
On institutional persistence
}

Citation for published version (APA):

Innocenti, S. (2018). On institutional persistence. [Doctoral Thesis, Maastricht University]. Datawyse / Universitaire Pers Maastricht. https://doi.org/10.26481/dis.20180328si

Document status and date:

Published: 01/01/2018

DOI:

10.26481/dis.20180328si

Document Version:

Publisher's PDF, also known as Version of record

\section{Please check the document version of this publication:}

- A submitted manuscript is the version of the article upon submission and before peer-review. There can be important differences between the submitted version and the official published version of record.

People interested in the research are advised to contact the author for the final version of the publication, or visit the DOI to the publisher's website.

- The final author version and the galley proof are versions of the publication after peer review.

- The final published version features the final layout of the paper including the volume, issue and page numbers.

Link to publication

\footnotetext{
General rights rights.

- You may freely distribute the URL identifying the publication in the public portal. please follow below link for the End User Agreement:

www.umlib.nl/taverne-license

Take down policy

If you believe that this document breaches copyright please contact us at:

repository@maastrichtuniversity.nl

providing details and we will investigate your claim.
}

Copyright and moral rights for the publications made accessible in the public portal are retained by the authors and/or other copyright owners and it is a condition of accessing publications that users recognise and abide by the legal requirements associated with these

- Users may download and print one copy of any publication from the public portal for the purpose of private study or research.

- You may not further distribute the material or use it for any profit-making activity or commercial gain

If the publication is distributed under the terms of Article $25 \mathrm{fa}$ of the Dutch Copyright Act, indicated by the "Taverne" license above, 


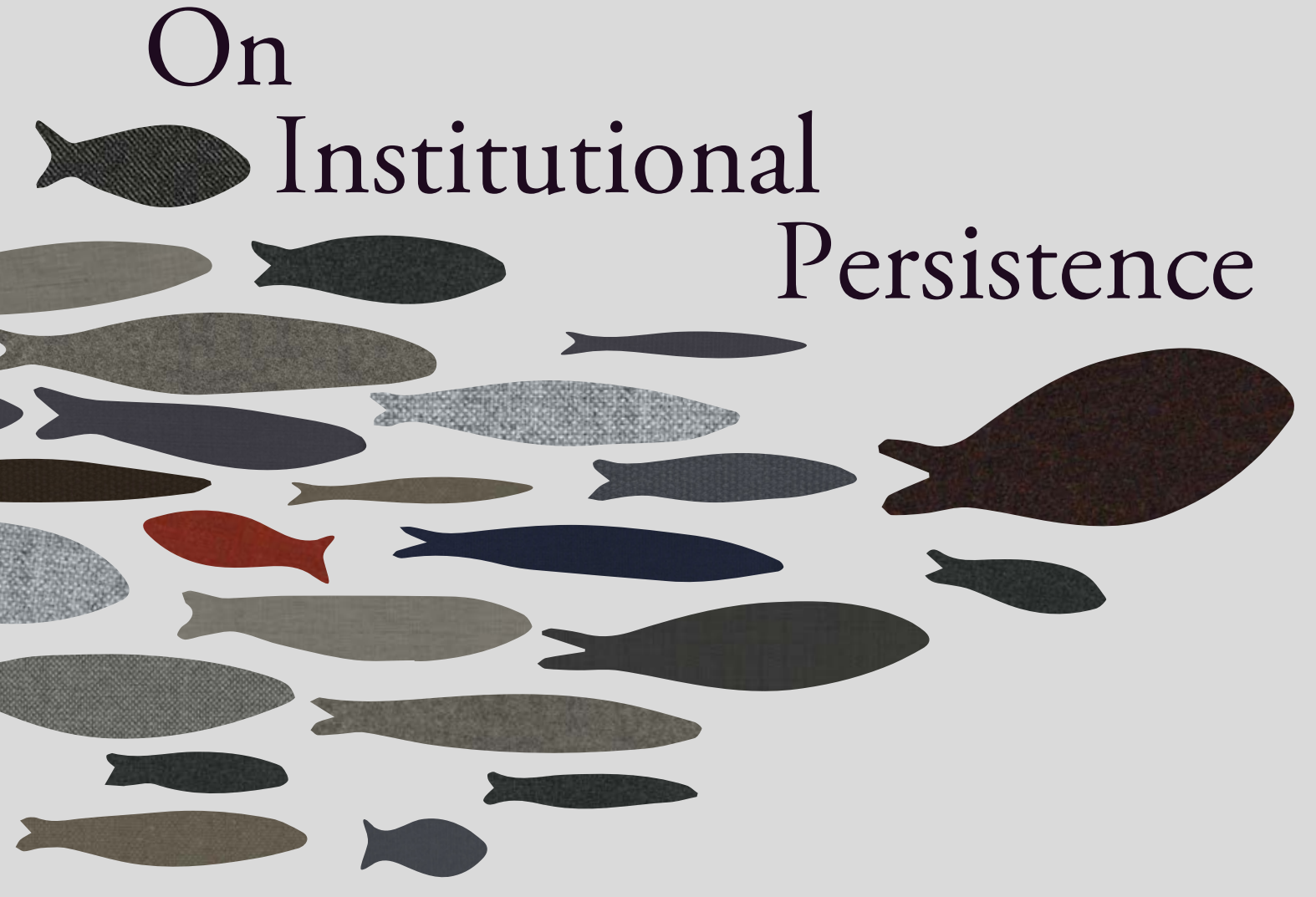

STEFANIA INNOCENTI 
(C) copyright Stefania Innocenti, Maastricht 2018

Printing: Datawyse | Universitaire Pers Maastricht

ISBN 9789461598165

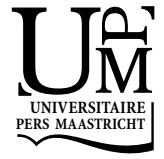




\section{ON INSTITUTIONAL PERSISTENCE}

\section{DISSERTATION}

to obtain the degree of Doctor at Maastricht University, on the authority of the Rector Magnificus, Prof. Dr. Rianne M. Letschert in accordance with the decision of the Board of Deans, to be defended in public on

Wednesday 28 March 2018, at 12.00 hours

by

Stefania Innocenti 


\section{Supervisor}

Prof. dr. Robin Cowan

Co-Supervisor

Dr. Ir. Eleonora Nillesen

\section{Assessment Committee}

Prof. dr. Théophile Azomahou, Maastricht University (chair)

Prof. dr. Michael Grimm, University of Passau

Associate Prof. dr. Martin Strobel, Maastricht University

Prof. dr. Marie Claire Villeval, CNRS University of Lyon 


\section{Summary}

The word institutions originates from the Latin verb instituere which means to establish, to erect, to be firm and unswerving. The etymology of the word hence testifies one of the main purposes of institutions: they are made to be solid and long lasting. It is their solidity that supports and possibly creates behavioural regularities transforming specific conducts into customary routines which facilitate social interactions.

By now a consensus has been reached on the fact that socio-economic institutions matter for economic development. Well-organised societies often prosper, whereas badly organised ones might go astray. Nonetheless, numerous empirical studies have shown that inefficient institutions have a strong tendency to persist. These instances require us to understand which factors hinder institutional change, leading to inertia and lowering the systemic development potential.

Whereas multiple studies have examined the phenomenon of change, few have focused their attention on institutional persistence. And more importantly, although most theories acknowledge that institutions are the results of individual decision-making processes, the link between human cognition, bounded rationality and institutional change remains understudied.

This thesis aimed to fill this gap and clarify the role of bounded rationality, learning, and beliefs as possible drivers of, or impediments to, institutional change. More specifically, there are two central questions which motivated this work: Do agents' cognitive structures affect whether or not institutional change occurs and how it unfolds? Can individuals' (in)abilities to enact change be responsible for institutional change (or the absence thereof)? The ultimate goal is to uncover the potential of self-efficacy perceptions as an explanation of institutional inertia.

To this end, I drew my analysis on three interpretative perspectives namely (i) the institutional economics principles on the importance of the rules of the game, (ii) quantitative evolutionary theories of change and learning dynamics, and (iii) the psychological investigation on self-views and their consequences on individual behaviour. I consider selfviews and institutional structures as parts of causal circularity along which each element descends from the above but also, in turn, determines it. Perceptions about ourselves, as well as about the broader environment, affect our desirability of rules' change. The rise 
of behavioural economics and its emphasis on the fact that agents are not highly sophisticated information processors, represents a good timing to deepen our understanding of institutional evolution.

Given its complex nature in order to be able to shed light on different aspect of the phenomenon under scrutiny, I rely in each chapter on a different method. After a review of the fundamental literature that concerns this study in Chapter 1, Chapter 2 describes the results of a laboratory experiment whose aim is to investigate whether a relation between self-efficacy beliefs and imitation exists. I designed a lab experiment which is a modified version of the common two-armed bandit with finite time horizon. Using data from one treatment, I study individual learning patterns using reinforcement learning models. In a second treatment, subjects are grouped in groups of 4 or 5 people. I investigate how individuals learn when given the possibility to observe the actions and rewards obtained by a randomly selected group "leader" who plays before everybody else. This allowed me to study the extent to which agents rely on observational rather than individual learning and how their propensity to imitate others is mediated by self-efficacy beliefs, which I measured using a standard psychometric scale. I find that, in stable environments, higher individual self-efficacy reduces the propensity to imitate the actions taken by the leaders. This implies that if people have little faith in their capabilities, they will follow the behavioural path set by somebody else. Consequently, imitating others might stabilise institutions and delay their change.

In Chapter 2, individual self-efficacy beliefs are assumed to be static. Although this is plausible in the context of a short lab-experiment, it might be a too simplistic approximation of reality. Therefore, in Chapter 3 I report the results of a simple binary choice model with social interactions and self-reinforcing dynamics. I frame institutional change as a binary choice between an old and a new behavioural rule. Agents can choose between option B, the status quo behaviour, and option A, the innovative one. The utility each agent obtains from the options depends on the intrinsic value of each alternative as well as on the choices made by others. In each period one agent is given the opportunity to revise his choice, and he does so based on a standard discrete choice model. The probability he will best respond depends on a stochastic exogenous parameter. The probability instead that any particular agent revises his decision depends positively on how much faith he has in his abilities, i.e. on his self-confidence or self-efficacy perception. Self-efficacy is endogenous, and changes depending on the success of one's past revision attempts. The reinforcement in self-efficacy and its effect on the probability that agents revise their state, represents the main methodological innovation of this chapter. The results show that a high heterogeneous distribution of self-efficacy beliefs within a population is likely to trig- 
ger institutional inertia. Low self-efficacious people prevent high self-efficacious ones from achieving a complete behavioural shift.

Despite the more realistic assumption concerning the dynamics of self-efficacy, Chapter 3 treats the evolution of institutions in quite an abstract manner. Chapter 4 takes a step further and uses the available secondary data to empirically investigate the relation between perceptions, institutions and ultimately economic growth. In the first part of the chapter, I focus on the nexus between mental models and institutional quality. The idea that mental models affect the institutional quality is not new. Yet, few scholars have carried out an empirical estimation. I use two different proxies for mental models' stickiness and panel data techniques to estimate their effects on institutional quality. I find that a ten percentage point decrease in mental model stickiness is associated with an improve a country's rule of law by 0.32 , possibly allowing it to move from the rule of Tunisia to the rule of law of Italy (in 2015). When controlling for country specific characteristics, this effect reduces by one order of magnitude. In the second part of the chapter, I test whether an independent effect of mental models and institutions on growth exists. Using a pooling model, I show that mental models considerably affect growth. Additionally, given the possible endogeneity problems, I use cross-sectional instrumental variables regressions and provide some weak evidence which suggests that in some cases mental models condition economic growth.

These three analyses use very different techniques, and approach the general issue from different levels, micro, meso and macro. They all, however, rest on the view that institutional change is connected to individual behaviour, and an important aspect of this connection runs through peoples' self-efficacy, or belief that they can improve their situations through their own actions. The variety of approaches and loci of analysis have generated results that are consistent with each other, and show that a coherent, multi-level view of how institutional change operates is possible. This thesis provides one example of this, and so lays the foundation for further research into an issue that is central to economic development and prosperity for many nations today. 



\section{Acknowledgments}

It is my pleasure to acknowledge the many people whose role was vital for the completion of my Ph.D research.

First and foremost, I would like to thank my supervisor, Prof. Robin Cowan. Without him this dissertation would not exist. His innovative ideas, skilful guidance, stoic patience and constant advice will stay with me for my entire life. He is undoubtedly a role model for me. I am also indebted to Dr. Eleonora Nillesen, my second supervisor, who was always there for me, ready to provide comments, support and share her experience and knowledge.

I would also like to thank the members of the assessment committee, Prof. Théophile Azomahou, Prof. Michael Grimm, Dr. Martin Strobel, Prof. Marie Claire Villeval whose valuable comments have enriched this thesis. I am also extremely grateful to Dr. Nicoló Bellanca to whom, at regular intervals, I turned to seek advice and suggestions, and to Prof. William Zame for his frank opinions on some of the chapters of this thesis. I also greatly appreciated the comments I received from Prof. Elisabet Rutstrom and the absolutely crucial conversation I had with Prof. Paul David in the early stages of this research project. I would also like to express my gratitude to Prof. Ray Duch for hosting me at Nuffield College in Oxford and to Dr. Huub Meijers who placed a bet on my teaching skills and passed down on to me extremely good advice on how to run tutorials. Lastly, I want to sincerely thank Prof. Giorgia Giovannetti who convinced me to start this Ph.D in the first place.

I truly enjoyed working in UNU-MERIT, a research environment which stimulates original thinking and initiative. Somebody described the institute as "a very warm coat in very cold winter". I could not agree more and I thank Ad, Mark, Wilma, Susan, Herman, Mourik-Ian for their constant technical support and, with all other members, for making MERIT such a special place. A truly special thanks goes to Eveline, the heart and soul of the Ph.D programme. There are hardly any words to describe my appreciation to her "free hugs and open door" policy.

My stay in Maastricht would not have been the same without all the friends who have accompanied me during this journey. Eva, Gintare, Biljana, Caroline, Saba, Raquel, Tigist, Thuy, Ayo, Tobias, Martin, Hasse, I could not have dreamt of a better batch. A 
very special thanks goes to Juan, Sheng, Daniel and Francesca who have always been there for me, any day, any time. I am also grateful to Alison, Elisa, Giulia, Eduardo, Giorgio, Jennifer T., Mueid, Hugo, Mario, Simone, George, Sachin, Iulia, Ibrahima, Elvis, Danilo, Beatriz, Josy, Tatevik, Hibret, Michael, Jennifer W., Omar, Iman, Charlotte, Shuan, Samyukta, and Alejandro for reminding me that there is life beyond a $\mathrm{Ph} . \mathrm{D}$ and there is always a reason for a Belgian white beer. A special thanks goes to Mira, Julieta and Ortrun with whom I shared office 1.13, the incubator of many of the ideas expressed in this thesis.

My last words go to my family. Without mom and dad, none of this would have been possible. There are no words to express my gratitude for providing me the freedom and understanding that was needed to write this book. Let this thesis be dedicated to them. Also thanks to Arianna, Luca, Sabrina, Mauro, Ale and Silvia for their constant support and the vivid discussions I had with them around the topic of this dissertation. I am indebted to my friend Francesca Ulivari for designing the cover of this book. Dulcis in fundo, my very final thank you goes to François, no doubt one of the best scientists I know. Thank you for all your help and for constantly reminding that, although difficult at times, this entire journey was absolutely worthwhile. 
1 Introduction 1

1.1 Institutions: rules or equilibria . . . . . . . . . . . . . . . . . 2

1.2 Institutional change . . . . . . . . . . . . . . . . . . . . . . . . 4

1.3 Institutional persistence . . . . . . . . . . . . . . . . . . . 5

1.4 Psychological perspective . . . . . . . . . . . . . . . . . . . 7

1.5 Knowledge gap . . . . . . . . . . . . . . . . . . . . 9 9

1.6 Methods ............................... 9

1.7 Thesis outline . . . . . . . . . . . . . . . . . . . . 10

2 Self-efficacy beliefs and imitation: a two-armed bandit experiment 13

2.1 Introduction . . . . . . . . . . . . . . . . . . . . . . . . . . . . . 14

2.2 Related literature . . . . . . . . . . . . . . . . . . . . . . . 16

2.3 General design of the experiment . . . . . . . . . . . . . . . . . . . 22

2.4 Theoretical models and estimation strategy . . . . . . . . . . . . . . 24

2.4 .1 Choice model . . . . . . . . . . . . . . . . . . 25

2.4 .2 Treatment one: Individual learning . . . . . . . . . . . . . . 25

2.4 .3 Treatment two: Imitation . . . . . . . . . . . . . . . . . 34

2.5 Results . . . . . . . . . . . . . . . . . . . . . . . . 41

2.6 Conclusions . . . . . . . . . . . . . . . . . . . . 44

2.7 Appendix: Monte Carlo Simulations . . . . . . . . . . . . . . . . 47

2.8 Appendix: Regression Tables . . . . . . . . . . . . . . . . . . . . . . 49

2.9 Appendix: Experimental Instructions . . . . . . . . . . . . . . . . . 53

3 How do institutions change? Self-efficacy Beliefs and Social Interactions 85

3.1 Introduction . . . . . . . . . . . . . . . . . . . . . . . . 86

3.2 Related literature . . . . . . . . . . . . . . . . . . . . . . . . 88

3.3 The model . . . . . . . . . . . . . . . . . . . . . . . . . . . . . . . . . 91

3.4 Results . . . . . . . . . . . . . . . . . . . . . . . . 93

3.4.1 Synchronous updating versus asynchronous updating with $\alpha=0$ : a comparison . . . . . . . . . . . . . . . . . 94 
3.4.2 Self-efficacy and asynchronous updating: the new rate equation . . 96

3.4 .3 Steady States . . . . . . . . . . . . . . . . . . . . . . . . . . 98

3.4.4 Collective welfare . . . . . . . . . . . . . . . . . 104

3.5 Conclusions and possible extensions . . . . . . . . . . . . . 105

3.6 Appendix 1: The decision tree . . . . . . . . . . . . . . . . . . 108

4 Mental models and Institutions $\quad 109$

4.1 Introduction . . . . . . . . . . . . . . . . . . . . . . . . 110

4.2 Related literature . . . . . . . . . . . . . . . . . . . . . . . . . 112

4.3 Data . . . . . . . . . . . . . . . . . . . . . 116

4.4 Mental models and institutions . . . . . . . . . . . . . . . . . . 119

4.4 Pooling the data . . . . . . . . . . . . . . . . . . . 119

4.4.2 Country and time fixed effect models . . . . . . . . . . . . . . 122

4.5 Mental models and growth . . . . . . . . . . . . . . . . . . . 127

4.5.1 Pooling, country and time fixed effects models . . . . . . . . . . . 128

4.5.2 IV regressions . . . . . . . . . . . . . . . . . . . . . . . 132

4.6 Conclusions . . . . . . . . . . . . . . . . . . . . . . . . 138

4.7 Appendix: Additional information and robustness checks . . . . . . . . . 140

$\begin{array}{lll}5 & \text { Conclusions } & 161\end{array}$ 


\section{CHAPTER 1}

\section{Introduction}

By now, most social scientists agree on the fact that institutions are extremely important as they are crucial determinants of economic phenomena and variation in longterm economic growth [Acemoglu et al., 2001, Rodrik et al., 2004, Szirmai, 2005]. Wellorganised societies prosper, whereas badly organised ones might go astray. Without money, there would be no trade. Without languages, communication would be extremely difficult. In the absence of defined property rights we would not know what is accepted to do with someone else's property and what to expect when the bounds are overstepped. If tax systems were not formalised, contributions to the common pool of state resources would be left to one's generosity. In a nutshell, institutions matter because they define incentives and thus condition the decisions to save, invest, innovate and adopt newly available technological artefacts.

In general, institutions should be relatively stable to facilitate the creation of order and thus promote economic development. Rule of law or any stable safeguard against predatory government behaviour, for instance, has proven beneficial for societies. Thus, its persistence is indeed an advantage rather than a problem. Nonetheless, some institutions persist over time, in spite of being no longer in line with - or conducive to - economic development. Their excessive resistance to change might reduce systemic flexibility and thus countries' development potential. Numerous empirical studies have shown that inefficient institutions have a strong tendency to persist. Unequal gender norms [Alesina et al., 2013] or contracted crop shares [Young and Burke, 2001] exhibit long-term inertia, despite the change in laws, technologies and in the agricultural market structures. The acceptance of corruption changes rather slowly [Roland, 2008] although it is by now clear that such practice erodes public confidence and undermines fair competition.

These instances require us to understand which factors hinder institutional change, leading to inertia and lowering the systemic development potential. Generally, the lack of institutional change is explained within the path-dependence framework according to 


\section{Introduction}

which when a historical trajectory has been selected, simply because of increasing returns to adoption, institutions can get locked-in regardless of the advantages of the available alternatives. This theory has proven very influential but one of its specific features casts doubts on its ability to fully explain the institutional dynamics.

The path-dependence framework relies on a simple utilitarian mechanism: agents are assumed to be rational and make their decisions on the basis of simple cost-benefit analyses. When the potential benefits of institutional change outweigh its costs, then actors are likely to perpetuate the current institutional configuration. The comprehensive rationality assumption stands in stark contrast with some of the findings proposed by the psychology literature which underlines that agents are not highly sophisticated information processors. Humans are instead boundedly rational [Kahneman, 2003, Simon, 1955], their beliefs affect their choices which might ultimately depart from optimality. According to Bandura [1997], for instance, human actions are crucially determined by individual self-efficacy beliefs, i.e. the beliefs people hold on their own capabilities to produce desired effects by means of their own actions.

In spite of the robust results obtained within the psychology domain, the role that individuals' self-perceptions and beliefs can play in the institutional change process remains understudied [Kingston and Caballero, 2009]. This represents the main gap that this thesis seeks to fill. More specifically, the ultimate objective of this thesis is to uncover the potential of self-efficacy beliefs as an explanation for institutional inertia.

This chapter elaborates on each step of general framework of the thesis. First, I review the existing definitions of institutions in the economic literature. I then focus on the theories of institutional change and persistence. I identify the research gap, spell out the methods used and lastly present the thesis outline.

\subsection{Institutions: rules or equilibria}

Within the institutional economics literature, two main strands of thought which hold different definitions have emerged. The first one sees institutions as rules whereas the second alternative approach sees institutions as equilibria.

The institutions-as-rules approach finds its origin in the work of North and his seminal book Institutions, Institutional Change and Economic Performance. North [1990] identifies institutions as the rules which guide the actions of individuals engaged in social interactions. The conception of institutions as rules is intuitive and well meets our habitual understanding of emblematic institutions: they regulate behaviour. Marriage, for instance, regulates rights and obligations of the members of the couple. Contacts enable exchange relations and regulate the commitments of the transacting parties. 
Differently from other constraints that limit human action such as the laws of physics, institutions are "humanly devised" and the reason for which these rules exist is fairly obvious. They "reduce uncertainty by providing a structure to everyday life" [North, 1990, p. 3]. Institutions guide interactions, thus when we need to greet a friend, drive a car, take a loan we have guidelines on how to execute these tasks. North [1990] includes in his definition both formal rules as laws, constitutions, contracts, and informal ones such as social and moral norms and conventions. While formal rules are designed and enforced by the state, informal ones are generally enforced by specific power groups.

Many scholars have since adopted some variants of North's definition whilst embracing the key characteristics: institutions are man-made rules which durably affect the way in which humans interact with one another.

Simply stating a rule though is not a sufficient condition to bring out an institution. There exist many rules that are not institutions per se as they are ignored by the majority of the population. A classic example is the one of traffic lights which are considered either prescriptions, or suggestions or simple decorations in some countries. According to the institution-as-equilibria approach the key element that transforms rules into institutions and induces people to follow them relates to the existing system of beliefs and expectations.

The institutions-as-equilibria approach focuses on the concepts of behaviour and of expected behaviour rather than on the rules. In a nutshell, according the institutions-asequilibria approach institutions are better conceived as formal and informal behavioural regularities that individuals have the incentive to follow as they represent the equilibrium solutions to coordination problems. It is the behaviour and the expected behaviour of others, rather than prescriptive rules, which induce individuals to behave (or not to behave) in a specific way [Greif and Kingston, 2011]. An institution is thus a structure which, in a precise social situation, leads individuals to regularly match their actions, creates expectations on the behaviour of others, and thus preserves itself. In fact, very often a rule of behaviour appears more attractive the higher the number of its adopters [Young, 1998b].

Within this approach scholars have defined institutions differently. Hayek [1973] and Schotter [1981] are considered the pioneers. In Hayek's [1973] view, institutions create regular patterns of behaviour and help people forming correct expectations and thus 'spontaneous order'. Schotter [1981] claims that agents repeatedly play certain games and develop rules of thumb, norms, conventions and institutions to interpret and frame other's actions. Calvert [1995, p. 22] claims that "institutions is just a name we give to certain parts of certain kinds of equilibria". Aoki [2001] defines institutions as a stable and shared system of beliefs which allows individuals to anticipate the behaviour of others in different 


\section{Introduction}

contingent situations. Greif [2006] sees institutions as beliefs, norms, and organisations which jointly lead to behavioural regularity. Young [1998a] maintains that institutions develop from the cumulative experience of individuals. Once individuals' interactions weld into regular expectations and behaviours, an institution has emerged.

Despite their subtle differences, all these definitions claim that institutions are coordination devices. If agents expect that the others will choose the equilibrium behaviour, then they will be motivated to act accordingly. Expectations represent the incentive to conform and, by conforming, agents induce other agents to conform too. Thus, the institution self-sustains: it becomes widely known and gets incorporated into agents' shared beliefs. The institutions-as-equilibria approach is able to explain why people act in accord to existing rules and why these rules emerge. People do not drive on the left or right side of the road simply because the rule in place says so, but rather because everybody does so and it is in their interest to align their behaviour to those of others if they do not want to crash.

The institutions-as-rules or equilibria approach provide a useful taxonomy to interpret and define institutions. And, as a matter of fact, the two approaches are considered by some [Guala, 2016], as complementary. But how do institutions change?

\subsection{Institutional change}

The different definitions of institutions also entail different views concerning institutional change.

Within the institution-as-rules approach, some authors treat institutional change as a centralised process [Libecap, 1989, Ostrom, 2005]. Rules are specified and revised either by the legislator or by any other collection of individuals which engage in collective action with the aim of capturing some benefits. According to these theories the change is the outcome of deliberate centrally driven choices. Others, instead consider that new laws or habits of thought are subject to uncoordinated selection processes. The variation in transaction costs is considered the driver of change [Williamson, 2000]. New institutional settings which are able to minimise transaction costs and align the incentives of the transacting parties will replace inefficient rules. Alternatively, Hayek [1973] postulated that institutional change happens because of a selection mechanism occurring at the group level. As more successful groups displace less successful ones, the institutions supported by the latter fade away.

Within the institutions-as-equilibria approach, institutional change is the result of a change in expectation. "A mere change in a statutory law is not an institutional change [...] unless it induces a qualitative change in the actual choices" and beliefs of people 
[Aoki, 2001, p. 233]. As a consequence, if an existing equilibrium dissolves, individuals will learn that their previously adopted strategies are not longer suitable. Thus, agents will start an experimentation process which, in the long-run, will lead to a change in expectations and behaviour. Eventually, agent's behavioural strategies and expectations will re-align and a new institutional equilibrium will be reached [Aoki, 2007]. Greif [2006] claims that individuals revise their expectation by observing each other's actions and outcome over time. Institutional change thus depends on individual learning experiences which ultimately lead people to converge towards a new rule.

Nevertheless, there exists evidence which shows that the institutional evolution might be complex. Sometimes institutions change regularly and at a fast pace. Some Latin American and African countries have often changed their political institutions over the last century, with recurrent switches between democracy and dictatorship [Acemoglu and Robinson, 2006]. Since its independence, Uganda switched from a parliamentary to a presidential system multiple times [Acemoglu and Robinson, 2008]. The Dominican Republic had 32 different constitutions between 1844 and 2008. In other occasions instead institutions prove very enduring. In England, the Magna Carta, signed in 1215, has shaped important parts of modern English Law [Roland, 2008]. In France, in 1799 a law forbidding women to wear trousers was approved. It was only in 2010 that ten French ministers proposed to repeal it. Although nobody had followed the rule for very long time, it was recognised as invalid by means of a parliamentary act only in 2012 [Guala, 2016]. In 2008, Egypt officially banned female genital mutilations. In case of infringement, the punishment should consist of a 3 to 15 years jail sentence. Nonetheless, according to the latest statistics, 8 years after the ban was approved, 33 percent of the Egyptian girls aged 0 to 14 have undergone female genital mutilations [Unicef, 2016].

Rules like the French ban on trousers or the female genital cutting ban in Egypt naturally lead to one question: under which conditions do ineffective institutions persist?

\subsection{Institutional persistence}

The phenomenon of persistence of ineffective institutions is often referred to as "the persistence puzzle" [Patterson, 2010]. The puzzle emerges from the fact that as mentioned above, in order to be effective and meaningful institutions should be stable and durable, but at the same time their excessive stability hinders change and consequently their effectiveness in regulating social interactions. Several elements have been recognised as possible obstacles to the institutional change.

Those who consider institutions as rules believe that group dynamics represents the main impediment to institutional change. Ostrom [2005] states that free-riding problems 


\section{Introduction}

can deter the initiation of collective actions to change the rules of the game. Libecap [1989] highlights that institutional structures might be affect by the redistribution of power. All groups whose interest is guaranteed by the current institutional configuration will strenuously attempt to impede any subsequent change. According to Olson [1965], resistance to change emerges because of the divergence in the objectives of the various interest groups.

Within the institutions-as-equilibria domain instead institutional persistence is considered the result of coordination [Young, 1998b]. Failure to coordinate is inherently costly, thus the positive feedback effect between individual and group behaviour generates persistence. Additionally, inefficient institutions might persist because of the asymmetry in the power size of the transacting parties [Hwang et al., 2013] or because of some forms of "institutional complementarity". Aoki [2001] underlines the existence of strategic connections between institutions across various domains of the economy. Institutional configurations are considered complementary when the viability of one specific institution is conditioned by the existence of others. For instance, the business model adopted by firms is strictly intertwined with the bank and insurance system in Japan [Aoki, 2001]. As each institution determines the constraints, incentives and ultimately agents' strategies, the dynamics of one institution depends on, and is reinforced by, any existing complementary institution [Amable, 2000]. When a web of complementaries is present, change is unlikely to occur in isolation. Rather, multiple complementary institutions should synchronously change and as this might be complex, institutions might endure in the long run.

In sociology, Patterson [2010] claims that institutional persistence is the result of social learning and imitation as these mechanisms allow inter and intra-generational reproduction of behavioural rules.

But there is one explanation of institutional persistence that gained widespread consensus amongst scholars belonging to both the institutional-as-rules and institutions-asequilibria approach. By now, many tend to identify history as the major cause of institutional resistance to change. According to North [1990, p. 98] institutions "evolve incrementally, connecting the past with the present and the future; history in consequence is largely a story of institutional evolution in which historical performance of economies can only be understood as a part of sequential study". Aoki [2007] claims instead that the equilibrium selection strategy, and thus the resulting institution, depends on the historically accumulated know-how that individuals hold. Thus, agents play a game which is contingent to historical factors [Greif, 1993]. This implies that when societies have embarked onto certain institutional paths, any present and future outcome is dependent on previously made choices. Small, and possibly random, past events can determine present and future institutional structures. 
At first, the institutional economics literature did not provide an explanation of the reasons why institutions, as biological organisms, evolve incrementally nor did it clarify why the past is so relevant to understand current institutional configurations [Gagliardi, 2008]. Given these evident gaps, the institutional economics literature endorsed the pathdependence theory, elaborated by David [1985, 1994] and Arthur [1989, 1994] to analyse technological trajectories.

The notion of path-dependence is meant to capture the way in which historically contingent events can generate self-reinforcing dynamics which might lock the economy into not necessarily efficient technological structures and pathways of development. There are three major analytical insights of the technological path-dependence theory which have helped understanding the reasons for which history deeply affects the evolution of institutions [David, 1994]. First, history shapes the formation of individual expectations which enable coordination to be achieved without centralised actions. Simply through the reinforcement of mutual expectations, accidents of history can become durable social arrangements. Second, there is a clear resemblance between institutions and the information channels through which technology diffuses: their role is to filter, compress and coordinate information helping any economic agent to take decisions. Third, drawing on a clear analogy with technological systems, institutions, in order to achieve consistency and compatibility, have to be strongly interdependent. Thus, specific historical events might lead to the adoption of one institutional rule and the effects of such decision overrun across complementary institutional domains.

The theory of path-dependence has undoubtedly filled an existing gap in the institutional economics literature. Nonetheless, several critiques have been ventured about the path-dependence framework. Martin [2010], for instance, has claimed that the fact that path-alterations are supposedly only possible as a result of unanticipated effects of external events limits the potential of the framework and reduces its evolutionary component. But the deepest critique relates to the minor role that individuals cognitive abilities play within this framework [Gagliardi, 2008].

\subsection{Psychological perspective}

Within the path-dependence model, the long-term reproduction of a given institutional pattern is explained with the notion of increasing returns to adoption. Any institution, once chosen, is supposed to deliver increasing benefits. Therefore, it becomes increasingly difficult to select alternative options, even if these alternative are more efficient. The pathdependence model thus relies on a simple utilitarian mechanism [Mahoney, 2000]. Agents make their decisions on the basis of a simple cost-benefit analysis. They are assumed 


\section{Introduction}

to be rationally able to run calculations with the clear aim to maximise some form of expected utility. Actors rationally opt for the current institutional structure because any potential benefit of transformation is outweighed by the costs.

The rationality assumption of the path-dependence model stands in contrast with some of the findings in the social psychology domain. Social psychologists have strongly highlighted that actors are not highly sophisticated information processors. Human behaviour is driven by instrumental reasons (i.e. cost-benefit calculations) as well as by non-instrumental ones. In fact, the human mind is a sense-making machine [Kahneman, 2011], its goal is to gain cognitive clarity. But clarity does not necessarily entail rationality. Humans are 'cognitive misers' and their actions might be result of emotions and self-views.

According to Bandura [1989] for instance, agents' ability to make decisions and enact them on the world is crucially determined by their own self-efficacy perceptions rather than solely by their comprehensive rationality. Self-efficacy is one of the main determinants of intention and has been defined as "people's judgments of their capabilities to organise and execute courses of action required to attain designated types of performances" [Bandura, 1986, p. 391]. Self-efficacy identifies the beliefs people hold on their own capabilities to produce desired effects by means of their own actions. Self-efficacy is not an endowed trait of personality. It rather develops over time continually integrating information from four different sources. First, self-efficacy beliefs change with the result of personal attempts to control contingencies. Second, self-efficacy beliefs change observing others and the consequences of their actions. Third, efficacy beliefs are influenced by verbal persuasion although in a less potent and enduring way than personal and vicarious experiences. Lastly, perceptions on one's ability to control reality is affected by emotional and physiological states [Bandura, 1997]. Self-efficacy perception affects individual motivation and action [Bandura, 1989]. Specifically, self-efficacy influences goal choice (i.e. increasing the probability of choosing an arduous goal), perseverance (i.e., increasing the probability that efforts will continue to be applied despite adversities), goal revision (i.e., increasing the probability that a goal will be revised upward after successful past performance), and goal-striving behaviour (i.e., increasing the effort applied to achieve the desired objective) [Bandura, 1997].

These psychological investigations cast doubts on the validity of the comprehensive rationality assumption of the path-dependence model. As a consequence its ability to explain institutional persistence is weakened. Social psychologists in fact claim that perceptions about ourselves affect decisions-making processes and the desirability of rule changes. Thus, institutional persistence might not be the result of simple cost-benefit analyses. Institutions might persist because of some basic human cognitive needs. In 
fact, no matter how defined, a consensus has been reached on the fact that institutions are the result of multiple decisions. Thus, human cognition and bounded rationality are likely to affect the evolution of institutions.

\subsection{Knowledge gap}

The link between cognition, bounded rationality and institutional change remains understudied. Kingston and Caballero [2009] in the Journal of Institutional Economics point out that

while many theories incorporate some form of bounded rationality, the precise range of roles that various forms of bounded rationality can play in institutional inertia and change remains unclear. Further theoretical and empirical work is needed to clarify the role of bounded rationality, of collective and individual learning and of endogenous preferences as drivers of, or impediments to institutional change. (p. 178)

This thesis starts from these premises. Its aim is to fill this gap and, relying on a theoretical framework based on elements from psychology and institutional economics, it investigates which role bounded rationality, learning, and self-views play in the institutional evolution. More specifically, there are two central questions which motivated this work: Do agents' cognitive structures affect whether or not institutional change occurs and how it possibly unfolds? Can individuals' (in)abilities to enact change be responsible for institutional change (or the absence thereof)? The ultimate goal is to uncover the potential of self-efficacy perceptions as an explanation of institutional inertia.

\subsection{Methods}

Given the inherent complexity of the phenomenon under scrutiny, in each chapter of this thesis I use a different methodology. More specifically, I employ a laboratory experiment, elaborate a simple computational model and use econometric techniques. Laboratory experiments are in fact useful to assess individual-decision making processes. Mathematical models and simulations help in the development of innovative frameworks and can be used to asses the logic of their underling hypotheses. Panel data are useful to assess the evolutionary dynamics of institutions. As a whole, these distinct tools allow me to propose a broad view of the institutional evolution (or lack thereof). 


\subsection{Thesis outline}

I investigate three plausible hypotheses which are able to explain why institutions stabilise and possibly persist.

As mentioned above, institutions delineate the set of incentives that individuals have to behave in a certain way while pursuing their objectives. According to North [1994], Aoki [2007] and Patterson [2010] the rate of change of institutions depends on individual learning rates. In fact, in order for institutional change to occur, individuals should come to believe that they could achieve better outcomes under a set of alternative rules of the game and that they can effectively bring about the change they seek. People learn which is the best decision to be taken either individually, simply sampling amongst the available alternatives, or observing others. One of the most common forms of observational learning is imitation. The great majority of the economic models depict imitation as an "economizing behavior". The standard argument in economics is that imitative behaviour occurs due to the decision maker's desire to simultaneously enhance his or her performance and save on learning costs. Psychology provides a complementary explanation. The decisions to imitate others depend on individuals' introspective beliefs about their own capabilities [Bandura, 1989], i.e. their own self-efficacy beliefs. According to Bandura [1997], copying the behaviour of others becomes appealing when one perceives oneself as unable to figure out how to accomplish a specific task. If the agent perceives him/herself as unable to take actions on his/her own, he/she will be more prone to rely on others' decisions. But, imitating somebody else's decision might delay or hinder the perceived need for a change in behaviour. And, this might mean that institutions do not wither away but rather stabilise. This argument is in line with Patterson's [2010] idea that social learning and imitation are the most common mechanisms of institutional reproduction. In Chapter 2, I test these hypotheses in an experimental fashion. The aim of the experiment is to investigate whether a relation between self-efficacy beliefs and imitation exists. My hypothesis is that weakly self-efficacious people, due to the low confidence in their own abilities, tend to follow the decisions taken by others. Conversely, more selfefficacious people might be less apt to follow others' actions and thus be more inclined to act independently and seek institutional change. This experiment allows me to derive some conclusions on the pace at which institutions change.

In Chapter 2, individual self-efficacy beliefs are assumed to be static. Although, this is plausible in the context of a short lab-experiment, it might be a too simplistic approximation of reality. In fact, as mentioned above self-efficacy changes over time as a result of, among other things, personal experience. For this reason, in Chapter 3, I investigate how experience-based learning and thus the dynamics self-efficacy affects institutional 
change. As mentioned above, the most widely held explanation in the literature is that institutional inertia is the result of increasing returns to conformity. In fact, widely diffused rules of behaviour are likely to attract additional adopters [Young, 1998b]. The positive externalities in adoption decisions emerge from advantages of shared behavioural expectations and thus a convergence of beliefs. In this chapter, I propose a complementary explanation. I posit that institutional inertia emerges not only as a consequence of increasing returns to conformity but also because of the way in which individual self-views evolve. If most members of the population do not believe in their abilities to change the status quo they are more likely to unquestionably follow the standing behavioural rule. I elaborate a simple discrete choice model with social interactions and self-reinforcing dynamics. Agents asynchronously decide between the status quo or an intrinsically more rewarding alternative. In line with the path-dependence framework, individual choices, aside from some possible mistakes, generally depend on rational calculations as well as on the peers' effects. Differently from other standard models in the literature, I assume that whether agents revise their decision depends on how much faith they have in their own abilities to ameliorate their welfare, i.e. on their self-confidence or self-efficacy perceptions. Self-efficacy is endogenous, and increases or decreases depending on one's previous successes or failures. I compare the case in which all population members have equal chances to enact the change to the case in which only few 'trendsetters' seize the available opportunities and assesses whether the former affects the diffusion of the institutional innovation.

Despite the more realistic assumption concerning the dynamics of self-efficacy, Chapter 3 treats the evolution of institutions in quite an abstract manner. For this reason, in Chapter 4, I take a step further and I empirically investigate the relation between perceptions, institutions and ultimately economic growth. In the first part of the chapter, I focus on the nexus between mental models and institutional quality. Mental models can be seen as the windows or spectacles through which individuals observe reality. In fact, mental models determine which behaviour is socially plausible and acceptable in a given situation. Ajzen [2002] posits that strongly prescriptive mental models reduce people's perceived behavioural control, i.e. one's freedom of choice and ability to control the outcome of one's actions. This suffocates intentions, triggers apathy and, over time, spirals into higher reluctance of changing the status quo. Therefore, one can expect that in case individuals feel that they have scarce freedom of choice and control over actions, they will not try to change the rules of the game. I bring this argument to the data and test whether mental model malleability affects institutional quality. The idea that mental models affect the institutional evolution can be traced back to Denzau and North [1994] and Mantzavinos et al. [2004]. Yet, few scholars have carried out an empirical estimation. 
I use two different proxies for mental models' stickiness and panel data techniques to estimate their effects on institutional quality. I also test whether mental models and institutions exert an independent effect on growth. And, to bypass endogeneity problems, I use cross-sectional instrumental variables regressions.

Chapter 5 synthesises the key results, conveys the larger significance of this study and sheds light on the research opportunities for extending the scope of this thesis. 


\title{
CHAPTER 2
}

\section{Self-efficacy beliefs and imitation: a two-armed bandit experiment ${ }^{1}$}

\begin{abstract}
In economics imitation is often considered a behaviour driven by payoff-enhancement motives. Psychology provides a complementary explanation: imitation becomes appealing when agents have little faith in their abilities. We investigate the extent to which selfefficacy beliefs affect agents' propensities to imitate others. We propose an experimental task, which is a modified version of the two-armed bandit. We measure participants' selfefficacy beliefs, then study individual learning. Subsequently, we measure how individuals use the information they gather observing a randomly selected group leader. We find that, in stable environments, a $1 \%$ increase in individual self-efficacy reduces the propensity to imitate others by $3 \%$.
\end{abstract}

${ }^{1}$ This chapter is based on Innocenti and Cowan (2016), UNU-MERIT working paper series. I would like to thank Eleonora Nillesen, Martin Strobel, Arno Riedl, Elisabet Rutstrom, Matthew Embrey and Nico Rasters for their advice. I also acknowledge the helpful comments of all the participants to the BEElab proposal meeting in Maastricht in February 2015, the ASFEE 2015 conference in Paris, the MLSE seminar in Maastricht in December 2015, the seminar at the University of Florence, IMEBESS 2016 conference in April 2016 and seminar at Nuffield college in Oxford in November 2016. This research was supported by the Institut Universitaire de France. The experiment was conducted following the peer-approved procedures established by Maastricht University's Behavioral and Experimental Economics Laboratory (BEElab). The study was approved at a public ethics review and project proposal meeting that is mandatory for all scholars wishing to use the BEElab facilities. 


\subsection{Introduction}

As we saw in the previous chapter, the literature is populated by a wide variety of definitions of institutions. All of these definitions concede that institutions represent the structure that regulates social interactions, they underpin (and embed) ways of thinking and acting. But how do agents get to know, within these structures, the best way to achieve their objectives? The vast majority of individual decisions occur in a routinised manner given the opportunities provided by the institutional framework in place [Nelson and Winter, 1982]. Nonetheless, sometimes in order to achieve their objectives, individuals have to behave in ways that seem contrary to the institutions that currently exist. Sometimes this simply involves a one-off violation of institutionalised norms of behaviour. Other times, though, it can involve movements towards changes in the institutions themselves.

Institutional change occurs because individuals learn that they could achieve better outcomes under alternative institutional configurations [North, 1994]. Rational choice scholars assume that agents are fully aware of their interests and objectives, and have full information about the environment. Institutional change is thus conceived as the result of a purely rational process [North, 1994].

But often individuals have incomplete information. Thus, they rely on simple trial-anderror learning in order to get to know what is the best decision to take under particular circumstances. Using the knowledge gained this way we can see individuals as updating their beliefs about different options based on their own past experiences. In addition though, learning can also occur through observing the successes and failures of others. Amongst the most common form of observational learning is imitation. ${ }^{2}$ Imitation consists in copying or mimicking the action of others. Imitation is widespread in the natural world. An animal can, for instance, avoid the costs related to sampling different feeding locations simply by imitating the actions of its conspecifics [Nicolle et al., 2011]. Humans also use imitation. For example, customer review websites and friends' advice facilitate learning

\footnotetext{
${ }^{2}$ Concepts like imitation, emulation and mimicry are highly debated in several disciplines which include neuroscience, psychology, anthropology, sociology and animal behaviour. These concepts mostly have to do with the act of copying somebody's action or performance. They all represent forms of observational learning. Nevertheless, subtle differences exist. Mimicry is defined as imitation for its own sake. It is considered the least cognitively demanding form of imitation. Imitation is a form of match dependent behaviour which though implies that a new action is being learned by observing another subject performing it. It also requires a purpose and a means/ends structure. Emulation instead implies that the subject observes somebody acting so as to achieve a goal and tries to achieve that same goal by whatever means. Imitation focuses on the means to a "static" goal whereas emulation focuses on which goal to pursue, having less to say about the means. Refer to Hurley and Chater [2005, pp. 1-52] for a detailed review of the literature on the topic. Although in the experimental design below actions are not new to the subject and thus we are not, strictly speaking, dealing with imitation, because subjects are focussed on the means to achieving the goal, we will with some approximation use the words imitation and observational learning interchangeably.
} 
through the positive and negative experiences of others. In all of these types of learning, which almost certainly co-exist, the learning can be about both specific actions within an institution, or actions explicitly designed to change institutions.

Theoretical and experimental work in economics depicts imitation as an "economizing behavior" [Alós-Ferrer and Schlag, 2009, Pingle and Day, 1996]. By copying others, the imitator minimises information-gathering costs, and information-processing costs. Thus, the standard argument in economics is that imitative behaviour occurs due to the decision maker's desire to simultaneously enhance his or her performance and save on learning costs.

This study takes a different approach to observational learning, providing a new perspective on the reasons for which individuals imitate. Building on contributions from psychology and economics, instead of questioning whether social learning occurs, we develop likelihood models to study the extent to which introspective beliefs affect observational learning. More precisely this study investigates whether individual self-perceptions of incompetence and aspiration-failure, i.e. low self-efficacy beliefs, influence imitation. Thanks to the recent work of Bénabou [2015] and Bénabou and Tirole [2016], self-efficacy has gained attention in the economics literature. Yet, no study within the economics literature has tested whether low self-efficacy beliefs affect individuals' propensity to undertake imitative behaviours.

We take some first steps to fill this gap. We use a modified version of the common two-armed bandit game with finite time horizon as the experimental setting. First in a laboratory, we measure individuals' self-efficacy beliefs. Subsequently, agents make a series of consecutive choices from which they derive real payoffs. In a first treatment, we study individual learning patterns. In a second treatment we observe how individuals learn when observing the actions and rewards obtained by a randomly selected group "leader" who plays before everybody else. This specific frame transforms the leader into a pioneer. Our goal is to understand how people process the information acquired by observing the leader's experience. Our hypothesis is that weakly self-efficacious people, due to the low confidence in their own abilities, use the leader as a role model and imitate his actions. Conversely, more self-efficacious people might be less apt to follow the leader's action and thus be more inclined to act independently.

Our analysis runs along the following lines. We (i) posit that subjects employ one of a set of individual learning strategies, (ii) for each strategy we derive the conditional probability of behaviour, given the available information, (iii) use these probabilities to generate likelihoods of the laboratory data, and (iv) select the strategy that best fits the data. Once we have studied individual learning we (v) study, following the same approach, how individuals learn observing others. Lastly, (vi) we investigate whether individuals' 
propensity to imitate correlates with self-efficacy beliefs. Rather than demonstrating its existence, we look for one possible cognitive reason for imitation. This is where our contribution lies. We find significant evidence to support our hypothesis: particularly in easy environmental conditions, higher self-efficacy beliefs reduce the propensity to imitate and induce higher reliance on individual learning processes. Results are inconclusive for more volatile environments.

The remainder of the chapter is organised as follows. Section 2.2 briefly reviews the literature on learning and imitation, making connections to the social psychology perspective. Section 2.3 presents the general design of our experiment. Section 2.4 presents our estimation strategy. We test our main hypothesis in section 2.5. Lastly, section 2.6 concludes and discusses the limitations of this study.

\subsection{Related literature}

Institutions regulate behaviour, reduce transaction costs and possibly lead to efficient outcomes. According to North [1994], the speed and direction of institutional change is a function of the individual learning rates. In 'normal' circumstance, individuals are assumed to make their choices and rationally seize the best available opportunities, and this will perpetuate the current institutional structure. However, it can happen that individuals come to believe that they could achieve better outcomes if the institutional structure were to change. This belief arises, clearly, from something that the individuals have learned (or believe they have learned) about the world. This learning can be 'rational', based on individual experience, or from observing others.

One of the best-known models of learning is the reinforcement learning model, also known as stimulus learning or rote learning. This model relies on payoff-based adaptive processes which are driven by the 'law of effect' [Thorndike, 1898]. Individuals are more likely to use those strategies that have resulted in greater payoffs in the past. In this model, players have limited rationality and do not need any information concerning the game being played. The payoff streams generated by past actions are the unique drivers of the learning process. This is formalised by associating to each strategy what in the literature is called prior, propensity of attraction, or attraction score. Propensities are reinforced over time using the newly obtained payoffs from playing the various strategies. Strategies are then played probabilistically, proportionally to their relative propensities. Models of this type were first used in mathematical psychology [Bush and Mosteller, 1951, 1953, Herrnstein, 1961, Luce, 1959]. With the unique exception of Cross [1973], the first applications in economics are mostly due to Arthur [1991, 1993] where he modelled learning agents in a financial market. Arthur's model is known as the one-parameter 
reinforcement learning model. In game theory, Roth and Erev [1995] and Erev and Roth [1998] modified the basic model to explain experimental evidence. Specifically they add two features to the basic model: recency and experimentation. Recency implies that recent events and experience affect future strategies to a greater extent than those of the distant past. Experimentation guarantees that players experiment with strategies that are similar to those that were successful in the past. This model has since been used in numerous experimental settings. In 2007, Erev and Roth compare the basic one-parameter with the three-parameters model and find that the latter outperforms the former.

Reinforcement learning models focus on the direct effects that obtained payoffs have on choice behaviours. These are among the most important drivers of human behavioural adjustments. Yet, as with most other animals, humans change their behavioural repertoire not only through personal experience but also by observing and possibly imitating others. Specifically, when it is possible to observe the actions and payoff obtained by someone else who is playing the same game as oneself, then following his behaviour becomes appealing. The simplest form of observational learning is mimicry: observing and mindlessly copying the actions of someone else. From the followers' perspective, the target becomes a point of reference or focus. A slightly more sophisticated form of observational learning is the one where the imitator uses the target's actions and their effects to gain extra information about the environment [Tomasello et al., 1987]. In this case observational and individual learning complement each other, and observational learning can in fact help confirm or disprove individual experiences [Bandura, 1989]. This behaviour allows individuals to reduce, in terms of effort and risk, the cost of individual trials and errors [Rendell et al., 2010]. It reduces the noise in the individually obtained estimates concerning the state of the world, and avoids making the same costly mistakes others have already made.

The learning literature in economics often claims that the motivation behind imitative behaviour comes from the discrepancies between one's own payoffs and the payoffs of others, i.e. when someone is performing better than oneself. Players are assumed to imitate in order simply to enhance their performance. Most theoretical work on imitation assumes that individuals copy the action of one other player, depending on his performance [Ellison and Fudenberg, 1993, Schlag, 1998, Selten and Ostmann, 2000, Vega-Redondo, 1997]. Specifically, Vega-Redondo [1997] and Selten and Ostmann [2000] assume that people imitate the successful action carried out in the immediate previous period. More generally, Alós-Ferrer [2004] assumes that people mimic the best performing action carried out in the past that the agent can recall. As for whom to imitate, people are often thought to follow the actions of the best performing competitor [Selten and Ostmann, 2000, Vega-Redondo, 1997], or of those that are just like them but playing against different opponents [Schlag, 1998] or, if the players have some preference for conformity, 
they follow the average choice of all other players [Bernheim, 1994]. The experimental literature has provided evidence that when given the opportunity to do so, individuals tend to imitate others. In general, people can either simply copy the observed behaviour or they can use this information to further their understanding of the decision-making environment. Several scholars have studied these phenomena. Huck et al. [1999] and Offerman et al. [2002] provide support for the Vega-Redondo [1997] model and both show that, in a Cournot game, when information on players' actions and payoffs is available, players tend to simply imitate better performers and the environment becomes competitive. In psychology, as early as 1941, the work of Miller and Dollard shows agents use the actions of others as sources of information, as inputs to a causal model. They show that observational learning is not much different from reinforcement learning, the main difference being that the stimulus is the behaviour of another person rather that one's own. This same theme has been taken up in experimental economics. Merlo and Schotter [2003] show that, in a Cournot game, observers learn better than subjects directly engaged in the task. Offerman and Sonnemans [1998] show that subjects imitated the forecasts of successful players. Apesteguia et al. [2007] show, also in a Cournot game, that agents' propensity to imitate more successful actions is increasing in the difference between the highest payoff observed and own score. Anderson and Holt [1997], relying on the models of Banerjee [1992] and Bikhchandani et al. [1992], show that when people are able to retrieve information both personally and through the observation of others, information cascades can occur. Knowledge stops accumulating, individuals stop using private information and simply copy the behaviour of their predecessors. So summarising, in economics theoretical and experimental work on observational learning starts from the assumption that the motivating factors behind imitative behaviours are related to some form of payoff enhancement objective.

Social psychology provides a complementary explanation. In general, it is thought that individuals do not engage in imitative behaviour as a result of a mere cost-benefit analysis. Humans imitate other's decisions and actions because of some specific cognitive needs. For instance Bandura [1989] argues that individuals' decisions, including those to imitate someone else, depend on their introspective beliefs about their own capabilities. The latter are referred to as an agent's beliefs in his or her own self-efficacy. ${ }^{3}$ These beliefs are crucial

\footnotetext{
${ }^{3}$ Self-efficacy is concerned with a person's perceived capabilities to achieve some goal. Self-efficacy differs from other concepts such as self-esteem, locus of control, or outcome expectancies. In his 1997 book Bandura claims that whilst efficacy is a judgment of one's capability, self-esteem is a judgment of self-worth. According to Bandura [1997, p. 11] "there is no fixed relationship between beliefs about one's capabilities and whether one likes or dislikes oneself". Even locus of control and self-efficacy are, according to Bandura [1997, p. 20], "entirely different phenomena". Locus of control is concerned with the beliefs that behavioural outcomes depend on one's own actions or on forces beyond personal control and "cannot by any stretch of imagination be considered the same as beliefs about whether one can produce certain actions (self-efficacy)" [Bandura, 1997, p.20]. Perceived self-efficacy is also
} 
for the decision to imitate others. According to Bandura [1997], copying the behaviour of others becomes more frequent than learning alone when one perceives oneself as unable to figure out how to accomplish a specific goal. If the agent perceives him/herself as unable to take actions on his/her own, he/she will be more prone to rely on others' decisions or actions.

Although Offerman and Schotter [2009] argue that "those who feel the need to imitate must, by definition, either not be able to do all the necessary calculations" or lack the inclination to do so, the role of introspective beliefs concerning one's capabilities as possible motives to imitate has not been explored within the economics literature. The recent contributions of Bénabou [2015] and Bénabou and Tirole [2016] have introduced the concept of self-efficacy. Together with other motivated beliefs, i.e. self-views, self-efficacy has been represented as an important element directly affecting people's preferences and ultimately their behaviour. However, no study in economics has focused on low self-efficacy beliefs as a possible reason to learn socially rather than individually, and thus undertake imitative behaviours.

This study takes a first step to fill this gap. We propose an alternative micro foundation of imitative behaviour. In order to copy a behaviour, individuals must have some motivating factors. These factors are related to the payoff enhancement that players envision but possibly also to their own self-evaluations of their capacities. In fact, the importance given to the actions carried out by the observed subject varies depending not only on the results he/she obtains but also on observer's self-efficacy ${ }^{4}$. While non-self-efficacious individuals use the observed subject as a role model, self-efficacious people seldom substitute individual learning with imitation. If agents believe themselves to have bad memory, to be unable to maximise their reward or understand the environment, i.e. to have low self-efficacy beliefs, they might be more inclined to imitate others independently from their performance. We therefore hypothesise that self-efficacious people imitate others to a lesser extent. Although, we have a priori no reason to believe so, we test, in an experimental fashion, whether this hypothesis holds in different conditions and thus depending on the difficulty of the task at hand. We use a first treatment of our experiment to estimate the model subjects use when their learning is based solely on their own experiences.

different from outcome expectancies. Self-efficacy has to do with people's confidence that they can perform a certain action if they wish to. Outcome expectations are judgments about the outcomes that are likely to flow from the performances one puts in place. They depict the expectations one has about outcomes given the behavioural choices one has decided to make (See Bandura [1997] on this specific point).

${ }^{4}$ Indeed, it might be argued that the weight individuals give to the action being performed by others depend on their preference for equality as payoff differences might trigger discomfort and thus imitative behaviour. Although we do not have experimental data for this specific aspect, in our final regressions we control for leaders' performance (i.e. score). This should take care of the eventuality that people imitate others just because they do not want their final score to be too different from that of those they observe. 
Then we focus on imitation. We investigate whether self-efficacy beliefs condition the propensity to copy a randomly selected leader, using models that integrate individual and observational learning.

We opted for an experimental task which is a modified version of the common twoarmed bandit problem with finite time horizon. The bandit problem takes its name from the common slot machines which can be found in casinos. In order to play, the gambler inserts a coin and pulls one of the machine's available handles (or arms) initiating the spinning of some flywheels. When the flywheels stop, a combination is displayed and the player receives a payoff. The subsequent gamble starts from the last combination obtained, nevertheless the player can choose to pull another of the available arms. At any trial, the gambler compares the scores obtained through the chosen handles. His objective is to maximise, over a series of pulls, the expected payoff. ${ }^{5}$

There are several reasons for choosing this experimental task. First, this setting allows us to assess how people make repeated choices. Moreover, by using a well-studied decision environment, we can begin with good candidate individual learning models and then isolate the effects of additional social information on observational learning processes. Lastly, binary choices lie at the core of the two-armed bandit. Although Simon underlined the importance of binary choice experiments to test, for example, utility-maximising principles as early as 1959, to the best of our knowledge this is a fairly unexplored experimental task [Banks et al., 1997, Burke et al., 2010, Efferson et al., 2006, Gans et al., 2007, McElreath et al., 2008, 2005, Nicolle et al., 2011, Schlag, 1998, Vostroknutov et al., 2017]. ${ }^{6}$ Only six studies have used this structure to investigate imitative behaviours. Schlag [1998] provides theoretical results which show that the best strategy to be played is to imitate the action of the observed individual with a probability proportional to the difference between one's own payoff and those of others in the previous round. Vostroknutov et al. [2017] use a two-armed bandit setting to study how intelligence levels affect how people learn from others. ${ }^{7}$ They find that participants with high intelligence use choices of others to better

\footnotetext{
${ }^{5}$ An optimal strategy for infinite time horizon bandit problems and exponential discount rates can be found calculating the Gittins index [Gittins, 1979]. However, this strategy is not optimal in our case because our problem has a finite time horizon and the type of discounting is for us unknown.

${ }^{6} \mathrm{~A}$ similar task is the well-known IOWA gambling task [Bechara et al., 1997]. Participants are presented with 4 decks of cards from wich they draw a card per round. Turning a card carries an immediate reward whose amount differs depending on whether decks $\mathrm{A}$ and $\mathrm{B}$ or decks $\mathrm{C}$ and $\mathrm{D}$ are chosen. Unpredictably, however, some cards also carry a penalty which is larger in decks A and B than in decks $\mathrm{C}$ and D. Players cannot predict when a penalty will arise in a given deck, nor they can calculate the profitability of each deck, and they are not given any information on how many cards they will be asked to choose. This game differs from the bandit games as the options available to the participants are in this case fours although the some decks share some of their characteristics. Additionally, the number of choices to be made is not known by the participants. Nonetheless, we acknowledge that modified versions of this task are not too dissimilar from our two-armed bandit task.

${ }^{7}$ They measure intelligence as efficient problem solving and abstract reasoning using Raven Advanced Progressive Matrices (RAPM).
} 
understand the decisional environment. Conversely, agents who score low on the intelligence test rely on mimicry. ${ }^{8}$ This study shares with ours the interest in uncovering the mechanism motivating imitation, but whereas Vostroknutov et al. [2017] claim that this mechanism can be found in the knowledge of one's own intelligence and that of the person we are imitating, we posit that confidence in one's capacities is key. Further, we measure self-efficacy beliefs, also controlling (to some extent) for intelligence by including individuals' math skills. On the presumption that more intelligent people are likely to be more successful in controlling their environments and so are likely to feel more self-efficacious, we could loosely equate [Vostroknutov et al., 2017, 's] intelligence measure with a proxy for self-efficacy. In the Burke et al. [2010] study, students engaged in a two-armed bandit experiment while being administered an fMRI scan. Before participants made their own choice, they observed the behaviour of another player who faced the same options. They found that when participants are informed both about the option chosen and payoff obtained by a peer, they choose the higher paying option at a significantly higher rate. They also find that agents tend to mimic a peer with a higher probability when only the choices made by the peer were observable relative to the case in which both actions and payoffs were observable. Nicolle et al. [2011] use a two-armed bandit to study the relations between observational learning and optimism. As observers do not directly incur costs or benefits during the learning process, observational learning is associated with optimistic over-valuations of low-value options. Our experimental design is closer to the work of McElreath et al. [2008, 2005] who, in the anthropology domain, used two-armed bandit experiments to study social transmission of behaviour and culture. Their experimental tasks were formulated as an agricultural choice. In the first treatment, individuals had to independently decide multiple times whether to plant one of two crops, 'wheat' or 'potatoes' in different farms. Farms differed from one another due to the variability of the yield [McElreath et al., 2008, 2005]. In a subsequent treatment, prior to making his/her crop decision each participant could access information on the most recent crop choice and yield of each member of his or her group. In another treatment instead, participants were given the possibility of observing the most recent planting decision, but not yield, of one single unknown member of their group. They use this treatment to study the evolution of human cultural traditions. They find that most participants tend to stick to the same behaviour if the target subject has made the same choice. We modify their treatment set-up to assess whether self-efficacy beliefs are a good predictor of imitative behaviours.

\footnotetext{
${ }^{8}$ Vostroknutov et al. [2017] define "simple imitation" in the way we define "mimicry", and "sophisticated imitation" as we define "imitation".
} 


\subsection{General design of the experiment}

Our experiment consists of three parts. First, subjects answered 10 questions aiming at measuring their problem-solving abilities and math skills. We selected some questions from the standard Graduate Record Examination (GRE) test. ${ }^{9}$ Subjects were allowed neither calculators nor pens and paper.

Second, our subjects were presented with a questionnaire to assess their self-efficacy beliefs. This is a standard questionnaire used to rate participants' confidence to perform certain behaviours in a set of hypothetical situations [Judge and Bono, 2001, Schwarzer et al., 1997, Schwarzer and Jerusalem, 1995]. It is not task-specific, it is rather meant to assess people's self-perceived potential and beliefs that actions are responsible for successful outcomes. The questionnaire consists of 10 questions. Students were asked to rate the extent to which each question applied to them on a 4-points scale which ranged from "Not at all true" (1) to "Exactly true" (4). The final score for each participant was obtained summing up all questions' scores. We treat this information as static and exogenous. We do not consider any feedback-loop between learning and self-efficacy beliefs. This seems reasonable given the short duration of the experiment.

Finally, subjects participated in the experimental game. Our game is divided into 2 treatments whose order was not randomised and which differ from one another mainly in terms of the information participants received. In treatment one, subjects sequentially chose between two alternative colours (1 and 2) for three sets of twenty rounds, thus making 60 binary choices. We will call a set of 20 rounds a sub-setting. The number of periods and sub-settings played were set to reduce the likelihood of participants growing bored and to have enough variation in the data to estimate learning patterns. To avoid people carrying their priors over sub-settings, every 20 rounds we changed the two colours between which subjects had to choose. Each sub-setting had a "preferred" colour, in the sense that it yielded a higher expected payoff. The preferred colour was set randomly at the start of the sub-setting, was the same for all subjects, and unchanged during the sub-setting. Participants were informed that there was a difference in expected payoffs to the two colours, but not what the expected payoffs were, nor which was higher. They were informed that payoffs ranged between 1 and 18 units. Subjects played by selecting one colour in each round and were paid in cash proportionally to the sum of the payoffs received. After agents selected their colour they were informed about the score received,

\footnotetext{
${ }^{9}$ In order to make sure that these questions were not too hard for undergraduate students, we ran a simple in-class pilot with 45 students. We administered the test to first year economics students enrolled in the International Economics course at Maastricht University. We checked the distribution of correct answered and fine-tuned the level of difficulty to make sure that on average people were able to answer at least 5 questions.
} 
and reminded which colour they had chosen. Only the most recent choice and payoff were displayed. Before the first round, no information was displayed. The payoffs of both options were drawn from normal truncated distributions, with fixed mean and variance, bounded between 1 and 18. ${ }^{10}$ Thus a sub-setting can be characterised by a quadruple: $\left(\mu_{1}, \mu_{2}, \sigma_{1}^{2}, \sigma_{2}^{2}\right)$.

The mean of the more rewarding colour, for instance 1 , was fixed and equal to $\mu_{1}=13$ units, while that of the less rewarding one was $\mu_{2}=10$ units. The variance was the same for both distributions and determined the difficulty of the decision-making environment. The three sub-settings were distinguished by their variances in the payoff distribution: $\sigma_{1}^{2}=\sigma_{2}^{2} \in\{0.25,4,16\}$. In principle, when the variance is low it is easy to learn which is the better colour. Conversely, when the variance is very high, detecting the more rewarding colour is more difficult. Each group of subjects played in all three sub-settings, but the sequence of variance values came in random order and changed across groups. ${ }^{11}$ The reason for opting for a dynamic task is the fact that in prolonged static situations little is being learned as nothing disrupts the execution of the activity.

Treatment two of our experiment differs from treatment one in the information given to participants. As in treatment one, the experimental task consisted of a repeated binary choice. Participants had to select one of two colours for three consecutive subsettings of twenty rounds each. Subjects were randomly assigned to a group of 4 or 5 people whose identity remained unknown. Each group had a leader who was randomly selected and faced the same environmental conditions as everybody else. Leaders played as in treatment one: they did not observe others' behaviour, only their own choices and payoffs were shown to them, exactly as in treatment one. Non-leaders had different information. For them this treatment resembles what Bikhchandani et al. [1992] define as an "observable signals" scenario. Leaders played first. Non-leaders were immediately informed of their leader's choice and payoff. Starting from round two onwards, non-leaders also observed the outcome of their previous personal choice as in treatment one. ${ }^{12}$ The leader is not necessarily the best performer, the most skilled or informed but simply the

\footnotetext{
${ }^{10} \mathrm{We}$ checked that the mean and variance in the sample of payoffs were close to those of the underlying normal distribution.

${ }^{11}$ The variance order was randomised at the group level. In fact, if we randomised at the individual level, patterns of imitation would have been difficult to detect in treatment 2 and the learning parameters would not have been easily compared.

${ }^{12}$ As shown in the experimental instructions presented at the end of the chapter (refer to Appendix 2.9), non-leaders were communicated the following information "Every person in this experiment is now a member of a group of 4 or 5 people randomly selected from all current participants. However none of you will know who the members of your group are. Everyone belonging to the same group will make his or her decision under exactly the same conditions, so the same colour is best for each person in the group. Each group will have a leader who will operate under exactly the same conditions as all other group members. The leader has been randomly selected. You will not be able to tell who the leader is. However, you will, in each round of each sub-setting of this part, observe what your group leader has just chosen and the reward he or she has obtained from his or her choice". As a consequence, there is
} 
one who explores the decision environment first. We opted for this design feature because it is not our goal to make claims about leaders' characteristics but rather about those of the followers.

As in treatment one, the payoffs were drawn from normal truncated distributions. The mean of the distribution from the most rewarding colour was set equal to 13 , whilst the one for the worse option to 10. The pairs of colours between which the individuals had to make a choice changed every twenty rounds, at the end of each sub-setting. One of the two colours was always on average better than the other. The best option was randomly decided and changed every sub-setting, as were the colours. The variance of the payoff distributions, as in the previous treatment, was set to be either low, medium or high. The order was randomised across groups. Monetary rewards, proportional to the total score each subject achieved, were given at the end of the session.

The experiment was programmed in PHP and administered via computer. All instructions were displayed on the screen (refer to Appendix 2.9). One hundred and seventy five undergraduate students (74 females and 101 males) participated in all parts of this experiment. The experiment was run at the BEELab at Maastricht University. It lasted about one hour and the average payment was of 18.2 euros, including a flat-rate show-up fee of 5 euros.

\subsection{Theoretical models and estimation strategy}

In order to test the above mentioned predictions and study imitative behaviours more specifically, we have first to study individual learning patterns. We observed the choices made and payoffs obtained in each round by each agent in treatment one. We select three plausible individual learning models. For each of the three prior-posterior updating rules, we estimate the individual learning parameter $(\beta)$ and select the best fitting model. Subsequently, we move to the analysis of treatment two whose goal is to understand how people filter available information concerning the behaviour of others. As we consider observational and individual learning as complements, we select two imitation models which respect this assumption. We will use the individual learning parameters that we estimated in treatment one. Using the vector of individual choices taken and reward obtained by agents in treatment two, we estimate the imitation parameters $(\alpha$ or $\theta)$.

no deception. Non-leaders are clearly made aware that their leader has been selected randomly and not according to any other specific characteristic. 


\subsubsection{Choice model}

In all the analysis that follows we use a standard logistic discrete choice model. The model assumes that each of the two possible strategies has a numerical evaluation, generally defined in the literature as "attraction score", which is updated in response to experience [Camerer, 2003]. We denote the attraction score of the option $i=\{1,2\}$ at time $t$ considering the choices made and payoffs obtained in each round as $A_{i, t}$. Thus the probability of choosing colour $i$ in round $t$ is written as:

$$
\operatorname{Pr}\left(i \mid \mathbf{A}_{t}, \boldsymbol{\Theta}\right)_{t+1}=\frac{e^{\beta A_{i, t}}}{e^{\beta A_{1, t}}+e^{\beta A_{2, t}}}
$$

where $\Theta$ is the set of free parameters, while $A_{1, t}$ and $A_{2, t}$ represent the "attraction scores" (see below for clarification) and more specifically the subjects' current estimates of the mean payoff of colour 1 and 2 respectively.

The logit model initially proposed by Luce [1959], is widely used in economics. Generally, it is meant to explain how best responding individuals maximise their expected payoffs based on the distribution of scores they obtained in previous periods. The parameter $\beta$ is usually interpreted as a measure of rationality [Blume, 1995]. The larger is $\beta$, the smaller the probability that the individual will deviate from the best response. When $\beta=0$ the agent chooses randomly between the two alternatives with probability 0.5 . As $\beta$ goes to infinity, the individual never deviates from the best response and the choice is, in that sense, optimal.

In our case, $\beta$ can be interpreted as the strength of the belief concerning the estimated average reward for each colour, or differently the strength of the belief of being correct. If $\beta=0$, the agent has little faith in his own estimation, the difference in the mean of the payoff distributions is neglected and the choice is made randomly. If $\beta$ goes to $\infty$, the agent firmly holds onto his estimates and the colour which is thought to be, on average, the most rewarding is always chosen.

\subsubsection{Treatment one: Individual learning}

We use data from treatment one to detect patterns of individual learning. More specifically, we are interested in uncovering the strength of individual belief of being correct when making binary choices, and in understanding how this relation changes depending on the environmental volatility.

This treatment can be considered "a black box" [Nax et al., 2016]. Players take actions and receive payoffs. ${ }^{13}$ No information apart from the result of the individual performance

\footnotetext{
${ }^{13}$ Differently from Nax et al.'s baseline case, in our case the payoff structure does not depend on others'
} choices. 
Table 2.1: Theoretical models

\begin{tabular}{ccc}
\hline Model & Updating rule & Free parameters \\
\hline $\begin{array}{c}\text { Running } \\
\text { Average }\end{array}$ & $A_{i, t}=\frac{N_{i, t-1} A_{i, t-1}+y_{i, t-1}}{N_{i, t}}$ & $\beta$ \\
\hline $\begin{array}{c}\text { Memory } \\
\text { Decay }\end{array}$ & $A_{i, t}=r A_{i, t-1}+(1-r) y_{i, t-1}$ & $\beta, r$ \\
\hline $\begin{array}{c}\text { Bayesian } \\
\text { Updating }\end{array}$ & $A_{i, t}=\frac{A_{i, t-1}}{\hat{\sigma}_{i, t-1}^{2}}+\frac{y_{i, t-1}}{\sigma^{2}}$ & \\
& $\hat{\sigma}_{i, t-1}^{2}+\frac{1}{\sigma^{2}}$ & \\
& & \\
& & \\
&
\end{tabular}

is provided to the players. Consequently, learning is the result of an asocial process. We consider this treatment as the best possible background to study individual learning.

There exist several methods that individuals could use to learn about the two available options, so we fit three different and minimally parametrised learning models which are presented in Table 2.1. The key variable in all cases is the attraction score for each of the two alternatives, and we use three models of how a subject can update the attraction scores in light of experience. This selection follows the previous literature and more precisely McElreath et al. [2005]. These are widely used models in the literature, but there is no a priori reason to believe one or the other is more likely to apply given our experimental task. Thus, we examine all three as alternatives. As mentioned above the attraction scores are the estimated average reward for each of the two colours. Table 2.1 shows the three learning rules that individuals might use to update their attraction scores.

The first model reported in Table 2.1, is a standard reinforcement learning model [Camerer, 2003, ch.6] and assumes that the individual updates the expected value of the mean of the payoff distributions simply calculating an average of the observed payoffs. At every period the agent adds to his prior $A_{i, t-1}$ the new payoff he obtained $\left(y_{i, t-1}\right)$ and normalises for the number of choices of kind $i$ he has made $\left(N_{i, t}\right)$.

The second model, as its name expresses, updates the estimated mean attributing heterogeneous weights $(r)$ to the observed payoffs. This second model contains the 'recency' feature that, as mentioned in the section 2.2, Erev and Roth [1998], Roth and Erev [1995] added to the standard reinforcement learning model. It is better known as an adaptive 
expectations' model or as a weighted running average. Thus, if $r=0$ information obtained from earlier rounds is completely ignored, and the estimate is equal to the most recent payoff observation. If $r=1$, the contrary applies. Lastly, under the third model, individuals estimate the distribution mean in a Bayesian fashion assuming that the longrun variance of the payoff distribution $\left(\sigma^{2}\right)$ is known. In this case, the importance of the most recent payoff obtained is a function of the estimated variance $\left(\hat{\sigma}_{i, t}^{2}\right)$ and the real long-run variance $\left(\sigma^{2}\right)$. For all models analysed, when colour $i$ is not chosen at round $t$, its estimated payoff mean is assumed to stay equal to previously formulated attraction score $\left(A_{i, t}=A_{i, t-1}\right)$. All models share the unknown parameter $\beta$ which can be estimated with maximum likelihood techniques. The Memory Decay model has an additional parameter, $r$, which needs to be estimated. Independently from the model analysed, the attraction scores are transformed into predicted choice probabilities using the standard logit model.

\section{Estimation of the individual learning parameter $\beta$}

The logit model described by Equation 2.1 gives the probability of observing the data conditional on the previous choices made and corresponding payoffs obtained. We can fit these models either on an individual basis - obtaining for each subject estimates for the parameters that maximise the likelihood of observing the vector of his or her choices - or across individuals, in line with McElreath et al. [2005], pooling the data together, and obtaining one value of the parameter estimate for the entire population of subjects. This is meant to provide general information on which model best fits the populationlevel vector of choices and thus indicate the relative proportions of strategies used by the participants' pool.

We first fit the models on pooled data, assuming all participants use the same updating rule. This produces, for each model analysed, a negative log-likelihood of observing the true data under the assumption that the model is true: $-\log \mathcal{L}(\boldsymbol{D} \mid x, \boldsymbol{\Theta})$ for model $x$ given the set of free parameters $\boldsymbol{\Theta}$ and where $\boldsymbol{D}$ denotes the data, a matrix containing the colours chosen by all participants over the 20 periods. The likelihood is defined as:

$$
\mathcal{L}(\boldsymbol{D} \mid x, \boldsymbol{\Theta})=\prod_{t=1}^{20} \operatorname{Pr}\left(D \mid \mathbf{A}_{t-1}, \boldsymbol{\Theta}\right)_{t}
$$

It is standard practice in likelihood estimations to take the natural log of all conditional probabilities and sum them:

$$
-\log \mathcal{L}(\boldsymbol{D} \mid x, \boldsymbol{\Theta})=-\sum_{t=1}^{20} \log \operatorname{Pr}\left(D \mid \mathbf{A}_{t-1}, \boldsymbol{\Theta}\right)_{t}
$$

We fit each model given in Table 2.1 to the data to retrieve the values of the parameter that maximises the joint likelihood of observing the pooled data $(\boldsymbol{\Theta})$. Parameter 
2 Self-efficacy beliefs and imitation: a two-armed bandit experiment

Table 2.2: Goodness of fit measures: pooled data

\begin{tabular}{|c|c|c|c|}
\hline Variance level & Low & Medium & High \\
\hline \multicolumn{4}{|l|}{ Run. Average } \\
\hline -Log-Lik & 1516.89 & 1995.33 & 2140.91 \\
\hline \multirow[t]{2}{*}{$\hat{\beta}$} & 0.63 & 0.50 & 0.49 \\
\hline & $(0.018)$ & $(0.018)$ & $(0.022)$ \\
\hline $\mathrm{AIC}$ & 3035.77 & 3992.67 & 4283.82 \\
\hline$\Delta$ & 0.37 & 0.18 & 0.12 \\
\hline$w$ & 0.03 & 0.00 & 0.00 \\
\hline \multicolumn{4}{|l|}{ Mem. Decay } \\
\hline -Log-Lik & 1512.28 & 1852.81 & 1994.63 \\
\hline \multirow[t]{2}{*}{$\hat{\beta}$} & 0.60 & 0.42 & 0.28 \\
\hline & $(0.026)$ & $(0.018)$ & $(0.020)$ \\
\hline $\mathrm{AIC}$ & 3028.57 & 3709.61 & 3993.27 \\
\hline$\Delta$ & 0.38 & 0.24 & 0.18 \\
\hline \multirow[t]{2}{*}{$\hat{r}$} & 0.61 & 0.26 & 0.40 \\
\hline & $(0.07)$ & $(0.03)$ & $(0.04)$ \\
\hline$w$ & 0.97 & 1.00 & 1.00 \\
\hline \multicolumn{4}{|l|}{ Bayes } \\
\hline -Log-Lik & 1520.69 & 2027.56 & 2237.38 \\
\hline \multirow[t]{2}{*}{$\hat{\beta}$} & 0.73 & 0.54 & 0.51 \\
\hline & $(0.021)$ & $(0.020)$ & $(0.029)$ \\
\hline $\mathrm{AIC}$ & 3043.39 & 4057.11 & 4476.76 \\
\hline$\Delta$ & 0.37 & 0.16 & 0.08 \\
\hline$w$ & 0.00 & 0.00 & 0.00 \\
\hline
\end{tabular}

Standard errors in parenthesis.

estimation is done through a numerical grid search. As mentioned above, we use flat homogeneous priors. We set the initial attraction scores equal to 9.5 for both colours. This is consistent with the experiment instructions that the possible payoffs ranged between 1 and 18 and that one of the two options was on average always more rewarding than the other. For the case of the Bayesian model, whose assumption is that agents also know the long-run variance of the payoff distribution. Therefore, we set, for each sub-setting, $\sigma^{2}$ equal to the real variance of the payoff distribution. ${ }^{14}$

We report in Table 2.2 the fits of each model on the pooled data for the three variance values. The parameter estimates are shown together with the estimators of the standard error of our parameters obtained taking the square root of the diagonal elements of the inverse of the Hessian matrix ${ }^{15}$. Table 2.2 also displays some goodness of fit measurements which allow us to compare the models scrutinised (see Burnham and Anderson [1998]).

\footnotetext{
${ }^{14} \mathrm{As}$ it can be seen from the experimental instructions, participants were not aware of the real value of $\sigma^{2}$, but could have possibly figured out if they were keeping track of their payoffs.

${ }^{15}$ These values are correct if the observations are independent, their number is large and the models are correct.
} 
The Akaike Information Criterion (AIC) for model $x$ is the natural logarithm of the likelihood of observing the data plus twice the number of free parameters $(k)$ in the model (i.e. $A I C_{x}=-2 \log \mathcal{L}(\boldsymbol{D} \mid x, \boldsymbol{\Theta})+2 k$.) There is no generally accepted threshold value for the AIC, smaller values indicate a generally better fit. In fact, the AIC provides a measure of the information lost by using model $x$ to estimate reality. $\Delta$ represents the predictive power of each model when compared to a random one. It is calculated as the ratio between the negative value of the log-likelihood of the model analysed, $x$, and the log-likelihood of a model wherein individuals choose randomly $\left(\Delta_{x}=1-\log (\mathcal{L}(\boldsymbol{D} \mid x)) / \log (\mathcal{L}(\boldsymbol{D} \mid\right.$ random $\left.))\right)$. The value of $\Delta$ varies between 1 , when the fit of model $x$ is perfect, and 0 when the fit is the same as the random model. This is a measure able to synthetically tell the proportion of variance in choice explained by the models, it is an analogue to the commonly used $R^{2}$. The Akaike weights, $w$, represent instead a way to make comparisons within a specific the set of models. The weight $\left(w_{x}\right)$ for model $x$ within a set of $n$ models (in our case 3 ) is calculated as follows:

$$
w_{x}=\frac{\exp \left(-0.5\left(A I C_{x}-A I C_{\min }\right)\right)}{\sum_{x=1}^{n} \exp \left(-0.5\left(A I C_{x}-A I C_{\min }\right)\right)}
$$

where $A I C_{\text {min }}$ represents the smallest $\mathrm{AIC}$ in the set of model considered. A possible way to interpret the Akaike weights is to think about them as the probability that a given model is correct, thus the highest value of $w$ corresponds to the best fitting model. The advantages of analysing the data in this way have been explained in multiple occasions [Burnham and Anderson, 1998, Gigerenzer, 2004, Gigerenzer et al., 2004] and mainly have to do with the possibility of comparing multiple competing hypotheses to explain the observed data.

By all the goodness of fit measures, Memory Decay is the best model. The AIC values for this model are the lowest regardless the higher number of free parameters. $\Delta$ values are the highest for all variance values. Although this measure does not account for model complexity, it is able to provide a rough guide of the variance explained by this model. Moreover, the Akaike weights $(w)$ show that the probability that people use the Memory Decay rule of updating, compared to the other available models, is approaching or equal to 1 for all variance values.

The estimates of $\beta$ show that, for all models considered, choices become more random with the increase of the variance in the payoff distributions. This implies that the extent to which individuals believe in their estimates of the payoff means, given the scores obtained from the two colours, decreases - as demonstrated by the declining $\beta$ - when the variance of the payoff distributions are high or the environment is highly volatile. This first result 
is in line with what was found by McElreath et al. [2005]. This pattern also emerges when looking at the dynamics of the frequency of correct answers per each variance value.

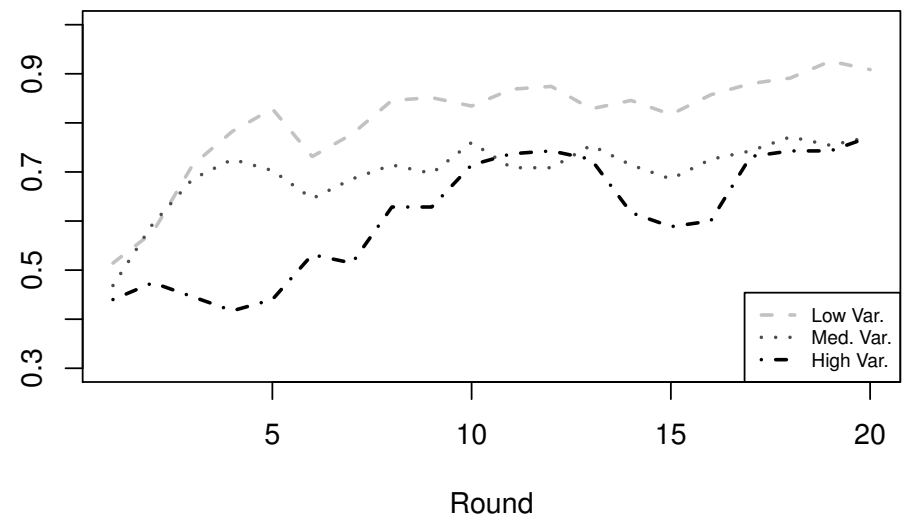

Figure 2.1: Share of correct answers per round in low, medium and high variance

Figure 2.1 shows that the proportion of correct decisions at each round declines with increasing task difficulty, i.e. variance value.

The decline of $\beta$ values per sub-setting is also found when analysing the ex-post elicitation of beliefs concerning the average reward of each colour. At the end of every sub-setting, participants were asked to give their best guess concerning the average reward obtained by choosing each of the colours. As can be seen from Figure 2.2, in case of low variance, the distribution of the answers is nicely peaked around the real means, indicated as a solid vertical line. Conversely, when the environmental volatility increases, some subjects have more difficulty in understanding which are the real, correct means of payoffs' distributions. 
Worse Color (Low Var.)

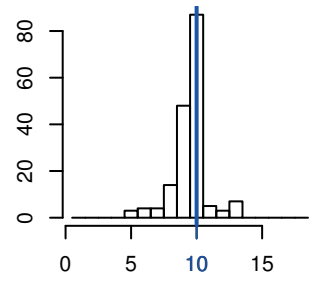

Best Color (Low Var.)

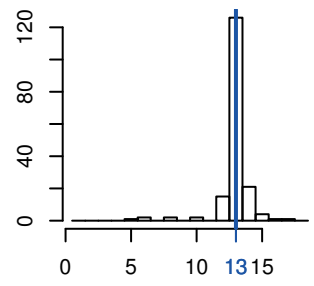

Worse Color (Med. Var.)

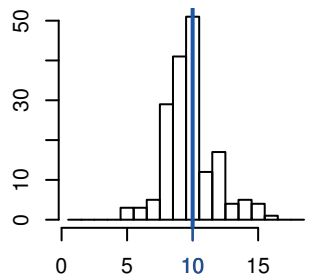

Best Color (Med. Var.)

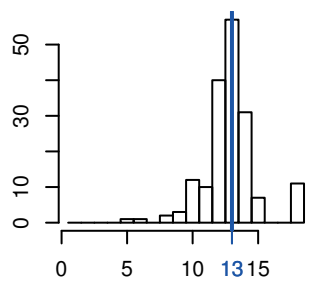

Worse Color (High Var.)

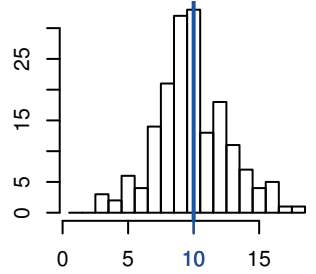

Best Color (High Var.)

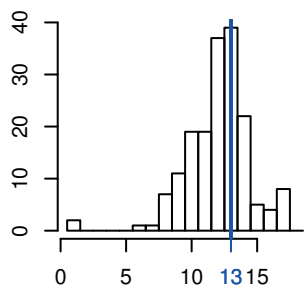

Figure 2.2: Distribution of posterior beliefs on the means of payoff distributions by variance value: Treatment 1

Table 2.2 also shows that the estimate of the $r$ parameter - the additional unknown parameter for the Memory Decay model - declines, although not monotonically, with increasing variance. In the high variance case agents pay more attention to recent events than they do in the low variance case. This shows that, contrarily to what one might think, in highly volatile environments individuals focus on recent signals and evaluate then as more valuable than past ones.

We also fit the models on an individual basis, treating the three models presented in Table 2.1 as possible alternatives to explain the choices made by each participant. This is what makes our analysis different from that of McElreath et al. [2005]. In this case, given the vector of choices made by each individual $j$ over 20 rounds $\left(\boldsymbol{D}_{j}\right)$ as well as the set of parameters to be estimated individually $\boldsymbol{\Theta}_{j}$, the $\log$-likelihood function for model $x$ can be written as follows:

$$
\log \mathcal{L}\left(\boldsymbol{D}_{j} \mid x, \boldsymbol{\Theta}_{j}\right)=\sum_{t=1}^{20} \log \operatorname{Pr}\left(\boldsymbol{D}_{j} \mid \mathbf{A}_{j, t-1}, \boldsymbol{\Theta}_{j}\right)_{t}
$$

We estimate the individual learning parameter $(\beta)$ for each model and each variance value $^{16}$, thus obtaining nine $\hat{\beta}$ per agent. In order to retrieve the values of the parameter

\footnotetext{
${ }^{16}$ We decided to estimate the individual learning parameter for each variance value because our objective is to determine which one of three possible models best explains the choices made by each participant. But the updating model chosen might vary depending on the degree of volatility of the decisional environment.
} 
that maximises the joint likelihood of observing each vector of individual data, we proceed numerically as we did in the pooled estimation case. We arbitrarily specify the upper bound of the grid within which search is conducted. It turns out that, in some cases, $\hat{\beta}$ takes values equal to the upper bound of this grid search, regardless of the value of the upper bound. These values might be divergent. This problem, which emerged particularly for the Memory Decay case, could be due to the fact that $\hat{\beta}$ is computed over only 20 rounds and thus unreliable estimates are produced. This implies that even if a true value of $\beta$ exists, simply because of statistical variation, our estimation strategy would be unable to estimate it correctly. Thus, in order to test our estimation method, we run 20000 Montecarlo simulations. We set the true $\beta$ and $r$ equal to the estimates obtained fitting the Memory Decay model to the pooled data as reported in table 2.2. We use the payoff distributions used in our experiment, and create fictitious data. We then fit the same Memory Decay model to these data. We check the distribution of the estimator $(\hat{\beta})$; in the case of low variance in the payoff distribution, in $26 \%$ of the cases $\hat{\beta}$ takes extreme values, diverging from the true $\beta$. In case of medium variance, the proportion of extreme values declines to $10 \%$, and is $11 \%$ when payoff volatility is high (see Appendix 2.7). These proportions are in line with what we observe in our experimental data. Our estimates are divergent in 37 cases $(21 \%)$ in the low variance sub-setting, in 31 cases $(18 \%)$ for medium variance and in $21(12 \%)$ cases when the payoff variance is high. This is not a new problem in this literature. The individual learning literature is well aware that in many cases the estimation method is unable to recover the true parameter values even when this is among those considered [McElreath et al., 2008]. As argued in Camerer [2003, ch.6], different studies have shown that the estimation of learning models can be disappointing if the number of possible strategies is small (e.g. 2 or 4) and the number of periods considered by the experiment is not long enough. Given these premises and the results of our simulations, we eliminate subjects whose estimated $\beta$ took on extreme values, and perform our individual learning analysis with a reduced sample size. ${ }^{17}$

A simple count shows that the Memory Decay represents the most frequently used updating rule. As shown in Table 2.3 this applies to over $68 \%$ of individuals in case of low variance, $83 \%$ in medium variance and $66 \%$ in the high variance case. Bayesian updaters

\footnotetext{
${ }^{17}$ We carefully checked the choices made by those individuals whose individual $\beta$ values are extreme. Amongst the excluded subjects, the proportion of those never changing colour is $37 \%$ (14 out of 37 individuals) in case of low, $22 \%$ ( 7 out of 31 individuals) in medium and $33 \%$ ( 7 out of 21 individuals) in high variance. The proportion of those instead that experiment less than 3 times corresponds to $64 \%$ in low, $67 \%$ in medium, $71 \%$ in high variance respectively. On average in low variance, the excluded people switched colours 2.67 times, while the remaining individuals experimented on average 4.9 times. In medium variance, the average number of changes for the excluded group is 2.61 versus 6.75 for all others. In highly volatile environments, while the average experimentation for the excluded group is 2.76 , the rest of the group switched colour on average 7.55 times.
} 
represent $24 \%$ of the sample population in low variance, $3 \%$ in medium variance and $20 \%$ in volatile environments.

Table 2.3: Number of individuals using the different models

\begin{tabular}{rccc}
\hline & Low Var. & Med. Var. & High Var. \\
\hline Running avg. & 9 & 20 & 20 \\
Mem. Decay & 95 & 120 & 102 \\
Bayesian Updating & 34 & 4 & 32 \\
\hline Total & 138 & 144 & 154
\end{tabular}

This result points to a wide usage of the Memory Decay updating rule among our agents. As a robustness check, in Table 2.4 we show the correlations of individual estimates of $\beta$ across models, once those individuals whose $\beta$ took on extreme values were excluded from the sample. The correlations are high for all variance values. More precisely, the individual estimates of $\beta$ obtained fitting the Memory Decay model are highly correlated with the estimates obtained fitting the other two candidate models. In both the low and medium variance cases the correlation coefficients are significantly around $70 \%$. In the high variance case, the correlation coefficients decline but remain above $50 \%$.

Table 2.4: Correlation Individual $\beta$ across models by variance value - excluding extreme $\beta$ values

\begin{tabular}{|r|cc|cc|cc|}
\hline \multicolumn{2}{|c}{ Low Var. } & & Med. Var. & & High Var. \\
\hline & Run.Avg. & Mem. Dec. & Run.Avg. & Mem. Dec. & Run.Avg. & Mem. Dec. \\
\hline Run.Avg. & & & & & & \\
Mem. Dec. & $0.75^{* * *}$ & & $0.70^{* * *}$ & & $0.69^{* * *}$ & \\
Bayes & $0.93^{* * *}$ & $0.69^{* * *}$ & $0.90^{* * *}$ & $0.81^{* * *}$ & $0.53^{* * *}$ & $0.51^{* * *}$ \\
\hline
\end{tabular}

As a robustness check we also repeated this same exercise while considering all individual $\beta$ values, including the most extreme ones. We report these results in table 2.5.

Table 2.5: Correlation Individual $\beta$ across models by variance value - including extreme $\beta$ values

\begin{tabular}{|r|cc|cc|cc|}
\hline \multicolumn{2}{|c|}{ Low Var. } & & Med. Var. & & High Var. \\
\hline & Run.Avg. & Mem. Dec. & Run.Avg. & Mem. Dec. & Run.Avg. & Mem. Dec. \\
\hline Run.Avg. & & & & & & \\
Mem. Dec. & $0.70^{* * *}$ & & $0.76^{* * *}$ & & $0.72^{* * *}$ & \\
Bayes & $0.95^{* * *}$ & $0.62^{* * *}$ & $0.93^{* * *}$ & $0.74^{* * *}$ & $0.38^{* * *}$ & $0.25^{* * *}$ \\
\hline
\end{tabular}

We also analysed within model correlation coefficients. In Table 2.6 we report the results obtained after the extreme $\beta$ values have been excluded. In Table 2.7 instead, as a robustness check, we report within model correlation coefficients when the entire sample is considered. The tables show that the individual learning parameter $\beta$ varies with the 
variance of the payoff distributions. Thus, we continue our analysis for the three levels of environmental volatility.

Table 2.6: Correlation Individual $\beta$ within models by variance value - excluding extreme $\beta$ values

\begin{tabular}{|r|cc|cc|cc|}
\hline \multicolumn{2}{|c}{} & & Run. Avg. & & Mem. Dec. & \multicolumn{2}{c|}{ Bayes } \\
\hline & Low Var. & Med. Var. & Low Var. & Med. Var. & Low Var. & Med. Var. \\
\hline Low Var. & & & & & & \\
Med. Var. & 0.10 & & -0.06 & & $0.22^{* * *}$ & \\
High Var. & 0.07 & $0.24^{* * *}$ & $0.21^{* *}$ & $0.28^{* * *}$ & $0.14^{*}$ & $0.17^{* *}$ \\
\hline
\end{tabular}

Table 2.7: Correlation Individual $\beta$ within models by variance value - including extreme $\beta$ values

\begin{tabular}{|r|cc|cc|cc|}
\hline \multicolumn{2}{|r}{} & Run. Avg. & & Mem. Dec. & \multicolumn{2}{c|}{ Bayes } \\
\hline & Low Var. & Med. Var. & Low Var. & Med. Var. & Low Var. & Med. Var. \\
\hline Low Var. & & & & & & \\
Med. Var. & $0.18^{* *}$ & & $0.26^{* * *}$ & & $0.21^{* * *}$ & \\
High Var. & $0.19^{* *}$ & $0.28^{* * *}$ & $0.26^{* * *}$ & $0.29^{* * *}$ & 0.10 & $0.18^{* *}$ \\
\hline
\end{tabular}

Given the simple count reported in Table 2.3 as well as the high correlation coefficients across estimated $\beta$, the Memory Decay model seems to have a clear advantage in predicting individual choices. Consequently we consider the individual $\hat{\beta}$ obtained by fitting this model to the data of treatment one to carry the rest of our analysis. ${ }^{18}$

\subsubsection{Treatment two: Imitation}

The goal of the second treatment is to understand whether and how people use newly available information concerning the choices and results obtained by a random group leader. As in the previous treatment we investigate which of the candidate models of imitation best predicts the decisional behaviours of our subjects.

This treatment can be considered an "observable signals scenario" [Bikhchandani et al., 1992]. A leader plays before everybody else, and his or her actions and payoffs are observed by his or her group members from round one onwards. Agents take action after the leader, and from round two on they are also presented their own choice and outcome from the previous round. ${ }^{19}$

As shown in Table 2.8, we restrict our analysis to two models of social learning. Both models are very simple and share two characteristics. Each player acts after the leader

\footnotetext{
${ }^{18}$ As a robustness check (not shown) we also carried our analysis with the individual $\hat{\beta}$ s obtained by fitting the first model presented in Table 2.1 to the data. We observed that the general results do not change.

${ }^{19}$ The leader sees only his or her own actions and payoffs.
} 
Table 2.8: Theoretical models

\begin{tabular}{ccc}
\hline Model & Updating rule & Parameters \\
\hline $\begin{array}{c}\text { Nested } \\
\text { model }\end{array}$ & $\operatorname{Pr}\left(i \mid \mathbf{A}_{t-1}, \boldsymbol{\Theta}\right)_{t} \propto(1-\alpha) L_{i, t}+\alpha X_{i, t}$ & $\alpha$ \\
\hline $\begin{array}{c}\text { Additive } \\
\text { model }\end{array}$ & $A_{i}^{\prime}=(1-\theta) A_{i, t-1}^{j}+\theta y_{i, t}^{z}$ & $\theta$ \\
& $\operatorname{Pr}(i \mid \mathbf{A}, \boldsymbol{\Theta})_{t}=\frac{e^{\beta A_{i}^{\prime}}}{Z}$ & \\
& $A_{i, t}=r A_{i}^{\prime}+(1-r) y_{i, t}^{j}$ & \\
\end{tabular}

has made his or her choice. This means that each group member, after his or her choices, has two observations from the same process with which to revise the means of the payoff distributions. Moreover both models assume that individuals weight the signals gained observing the leader and possibly use them to validate or invalidate private information.

First, in line with McElreath et al. [2005] this situation can be modelled using a nested probability model. The model's name, i.e. nested probability model, recalls the terminology used in previous literature [McElreath et al., 2008, 2005] and clearly expresses the fact that we consider previously estimated probabilities and plug them into this model. Specifically, the probability of choosing colour 1 at time $t$ is given by

$$
\operatorname{Pr}\left(1 \mid \mathbf{A}_{t-1}, \boldsymbol{\Theta}\right)_{t} \propto(1-\alpha) L_{1, t}+\alpha X_{1, t}
$$

where $X_{1, t}$ is an indicator variable taking the value 1 if the leader chose colour 1 at time $t$, and is 0 otherwise. $L_{1, t}$ is the probability that the subject would choose colour 1 at $t$ were he playing alone, as defined in Equation 2.1. This probability is calculated in a conservative manner. We rely on our previous estimates from treatment one, using the individual estimates of $\beta$ and $r$ obtained by fitting the Memory Decay model to the data from treatment one. The relevant unknown parameter to be estimated in Equation 2.6 is $\alpha$. This parameter measures the individual propensity to imitate. If $\alpha$ is zero, the model reduces to the simple individual learning process. The agent fully relies on individual learning and any information provided by the leader's action is dismissed. If $\alpha$ is 1 , the individual mindlessly mimics the behaviour of the target subject. Intermediate values of $\alpha$ can be interpreted in two ways. $\alpha$ can represent the propensity of a subject to mimic the leader, which is equivalent to ignoring his or her own information. Alternatively, equation 2.6 can represent a reduced form observational learning model, in which $\alpha$ captures the 
importance the subject puts on social observation. In either case, whether mimicry or imitation, we hypothesise that the value of $\alpha$ will be related to a subject's self-efficacy beliefs.

Second, imitation is modelled as an additive process according to which individuals add the information retrieved from the leader's action to their private information. The principle here is that the quality of the information gathered from the observation of the leader is exactly the same as that of any other agent. The leader's signal is not a priori any better than anyone else's. Thus, at the end of round one, group members hold two pieces of information from the same payoff distribution. The relevant unknown parameter to be estimated is, in this case, $\theta$ which measures again the individual propensity to imitate. More precisely, $\theta$ captures how much individuals value the experience of the leader. In this case, for estimating the parameters, the sequence we assume is the following. At the start of each round the subject has, for each colour, 1 and 2, a prior belief of the mean payoffs $\left(A_{1}, A_{2}\right)$. The leader (identified as subject $z$ ) plays and the non-leader (identified as subject $j$ ) observes the leader's choice. Suppose that the leader has chosen colour 1 , and has obtained a corresponding payoff $y_{1, t}^{z}$. According to the updating rule of Table 2.8 , subject $j$ updates his estimate of mean payoff for colour 1 to $A_{1}^{\prime}$. Based on the pair $\left(A_{1}^{\prime}, A_{2}\right)$ the subject makes his choice following the standard logit model presented in Equation 2.1 and reported in Table 2.8 where $Z=e^{\beta A_{1}^{\prime}}+e^{\beta A_{2}}$. We use the previously estimated individual $\beta$ to calculate this probability. Suppose subject $j$ chooses colour 1 . He observes the payoff he obtained $\left(y_{1, t}^{j}\right)$ and updates his estimate of the mean payoffs to a new pair $\left(A_{1, t}, A_{2, t}\right)$ following the Memory Decay rule (see Table 2.1). As discussed in the previous Section, statistically, the Memory Decay model works best for updating based on individual information. Thus in this step we use that rule, applying for each subject the value of Memory Decay, $r$, fitted from the first, individual play, treatment. We assume that in period $t=0$ our agents start off with flat priors (uniform on $[1,18]$ ), on the unknown mean of the payoff distributions. ${ }^{20}$

We fit the two models to the individual data from treatment 2 and estimate the two imitation parameters for each variance value.

First we report simple information concerning the choices made by our subjects in treatment 2 .

When comparing the vector of "correct" answers that all individuals provided in treatment one, (individual play), with those of treatment 2, (play with a leader), it can be seen that on average performance increases (see Figure 2.3). The share of correct answers per round in treatment 2 is generally higher than that observed in the individual treatment

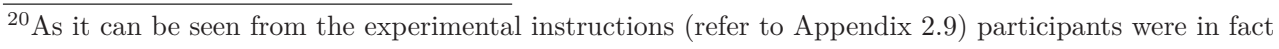
told that "Possible scores range from 1 to 18 ".
} 
both in case of low and medium variance. ${ }^{21}$ In the high variance case, the effect of playing with a leader is unclear. Signals are mixed, and while the payoff distributions had the same moments both with and without the leader, there is no temporal pattern. This is almost certainly driven by the fact that in the high variance case, subjects were simply unable to detect which colour was superior, due to the large noise in the signals they receive. Information could not be extracted either from their own signals or from the signals given by the leader's play.

\footnotetext{
${ }^{21}$ One might wonder whether the increase in performance might be a simple artefact of the experimental design. Specifically, it could be argued that in treatment 2, and more specifically in low volatile environments, both leaders and followers improved their ability to choose the best colour as they have already performed this task. Thus, their choices more frequently coincide. Nonetheless, we believe that the randomisation of the variance values as well as the change in the colours should deter strong learning effects and thus carrying over priors from one treatment to the other. Additionally, as shown in Figure 2.4 leaders who play on their own twice do not seem to evidently increase their performance. Indeed, simultaneous convergence towards the right colour cannot be excluded. But the evidence explored does not seem to suggest that this is due to individual learning across treatments.
} 
2 Self-efficacy beliefs and imitation: a two-armed bandit experiment
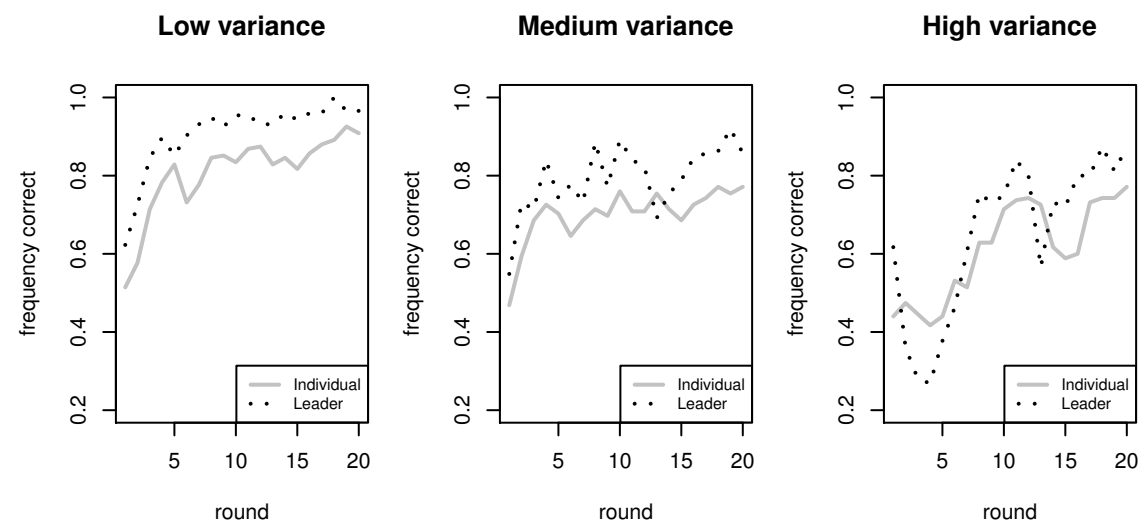

Figure 2.3: Comparison correct answers per round by variance: Individual and Leader treatment 

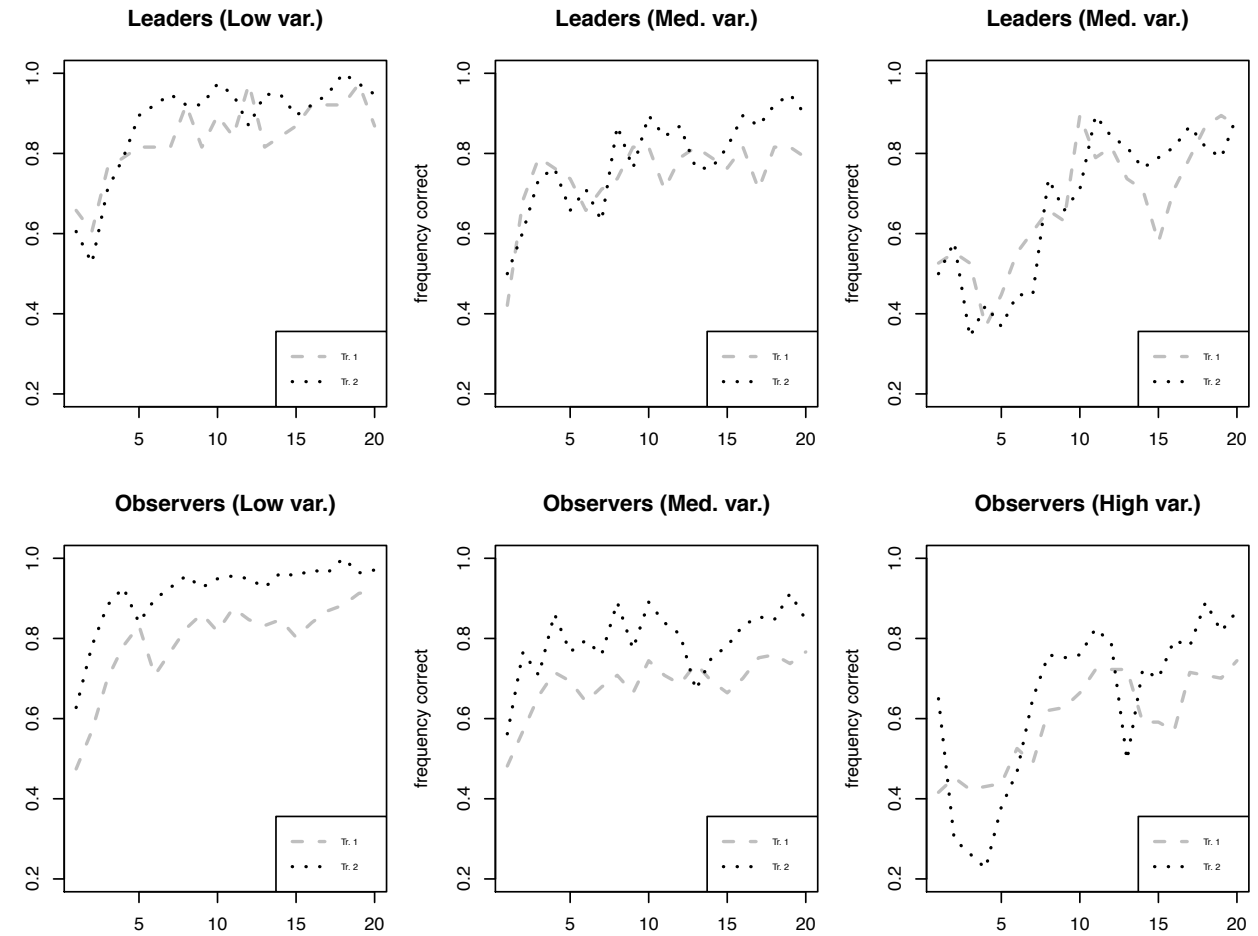

Figure 2.4: Comparison correct answers per round by variance of leaders and observers in treatment 2 
In order to gain further insights on whether the performance increase observed in Figure 2.3 is really due to social learning, in Figure 2.4 we separate leaders from non-leaders (i.e. observers) and we compare the share of correct answers for the two groups in both treatments.

Figure 2.4 clearly shows that whereas the performance of observers in treatment 2 increases visibly - especially in the low and medium variance cases - the leaders' performance in the second treatment of the experiment, albeit a slight improvement, follows the same trend observed in treatment $1 .^{22}$ Thus, we believe that this shows that observers have clearly improved their performance due to observational learning.

We also report, in Figure 2.5, the distribution of posterior beliefs on the means of the payoff distributions retrieved by end-of-sub-setting questionnaires. After 20 rounds, students were asked to give their best guess of the average rewards given by the 2 colours they had to choose from. The same pattern seen in Figure 2.2 is present. In a more volatile environment, fewer individuals correctly guess the real means of the payoff distributions.

\footnotetext{
${ }^{22}$ We also re-estimated individual $\beta$ parameters using the participants' choices in treatment 2 . We used the same grid-search described above and ran this exercise for the first and second model presented in Table 2.1 only. We compared these values with the estimates obtained using data from treatment 1 and check for their correlation. As for the population of leaders $(n=38)$, the estimated obtained using Model 1 are positively correlated but only significant for the medium variance case (the Pearson correlation coefficients are $0.22,0.19$ and 0.27 for low, medium and high variance respectively). As for non-leaders $(n=137)$, the correlation coefficients obtained using the same model are also positive and significant for both low and medium variance $(0.18,0.16,0.10$ for low, medium and high variance respectively). When we consider all people $(n=175)$ these coefficients are all significant and positive for all three variance values $(0.19,0.16,0.13$ for low medium and high variance). When estimating the individual learning parameter using the Memory Decay model instead we observe more or less the same pattern. The Pearson correlation coefficients for the leaders' population are positive and significant only for the medium case (correlation coefficients are equal to $0.11,0.34$ and 0.18 for low, medium and high variance). For non-leaders these coefficients are equal to 0.17 (low), 0.25 (medium), 0.13 (high). When we consider the entire subject pool we obtain significant correlation coefficients which are equal to $0.15,0.26$ and 0.14 for low, medium and high variance respectively. The low correlation for leaders, who play the same game twice, might cast some doubts on the stability of the $\beta$ parameter per se. As a consequence, as a robustness check we re-run the entire analysis. We first re-estimated the individual learning parameter relying the vector of choices made by our participants in Treatment 2. We then use these results to re-estimate individual propensities to imitate others (i.e. the parameter $\alpha$ ). Lastly, we ran the main regression analysis of the paper and checked whether selfefficacy beliefs remain still a good and significant predictor of people's propensity to imitate others. We focused only on the low variance case, and only on the Memory decay model (Model 2) but our results are in line with what is shown in table 2.9. After having excluded the leaders as well as those individuals whose $\beta$ takes on extreme values, the coefficient of log self-efficacy has the same magnitude $(-3.14)$ and stays significant.
} 

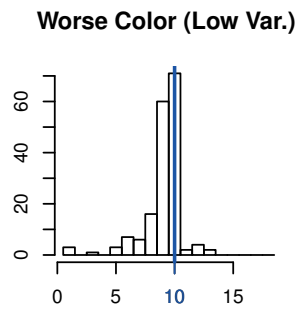

Best Color (Low Var.)

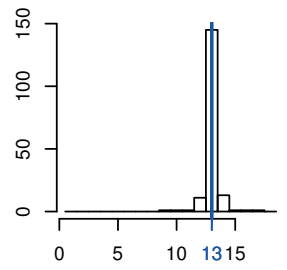

Worse Color (Med. Var.)

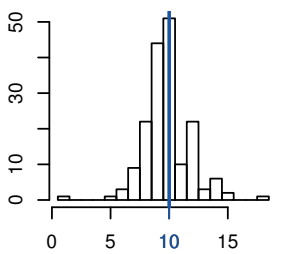

Best Color (Med. Var.)

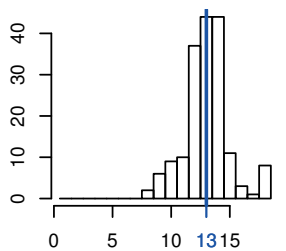

Worse Color (High Var.)

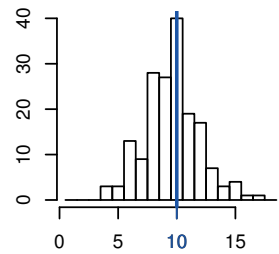

Best Color (High Var.)

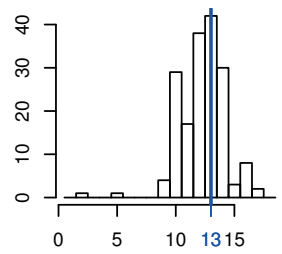

Figure 2.5: Distribution of posterior beliefs on the means of payoff distributions by variance value: Treatment 2

\subsection{Results}

The last step of our analysis concerns the relation between the propensity to imitate and self-efficacy perceptions. Specifically, we ask whether self-efficacious people 'imitate' a peer and how this changes depending on environmental conditions. As mentioned in Section 2.2, drawing on the psychology literature, one would expect a negative relation between propensity to imitate and self-efficacy beliefs.

In Table 2.9, we report the results of our preferred OLS regressions. Our dependent variable, i.e. the logarithm of $\theta$ or $\alpha$, captures individuals' propensity to imitate, or the weight subjects give to leaders' actions in making their own choices. The logarithm of the results of the self-efficacy questionnaires represent our main regressor. We include two controls. The first is math ability. If a problem-solving environment is difficult for a subject, we might expect a higher reliance on a leader for information and learning. This would be in line with the work of Vostroknutov et al. [2017] according to which individuals rely on simple or sophisticated imitation depending on their intelligence. Therefore, to control for subjects' ability to solve the problems implicit in the experimental environment, we used the scores they achieved on the math test we administered. Second, one might argue that copying the leader's action would be more likely if the leader is achieving high payoffs. This is a standard argument within the literature. For this reason, we control for the score obtained by the group leader. 
2 Self-efficacy beliefs and imitation: a two-armed bandit experiment

In this regression analysis we excluded the 38 leaders (since they are playing individually and have no leader to follow or not) and those whose $\beta$ estimates were at the extreme values, as discussed in Section 2.4.2. ${ }^{23}$ Thus, the sample size reduces to 109, 113 or 122 people depending on the variance value.

$\overline{{ }^{23} \text { In some cases, the individuals whose } \beta}$ values took on extreme values are also leaders in treatment 2. 


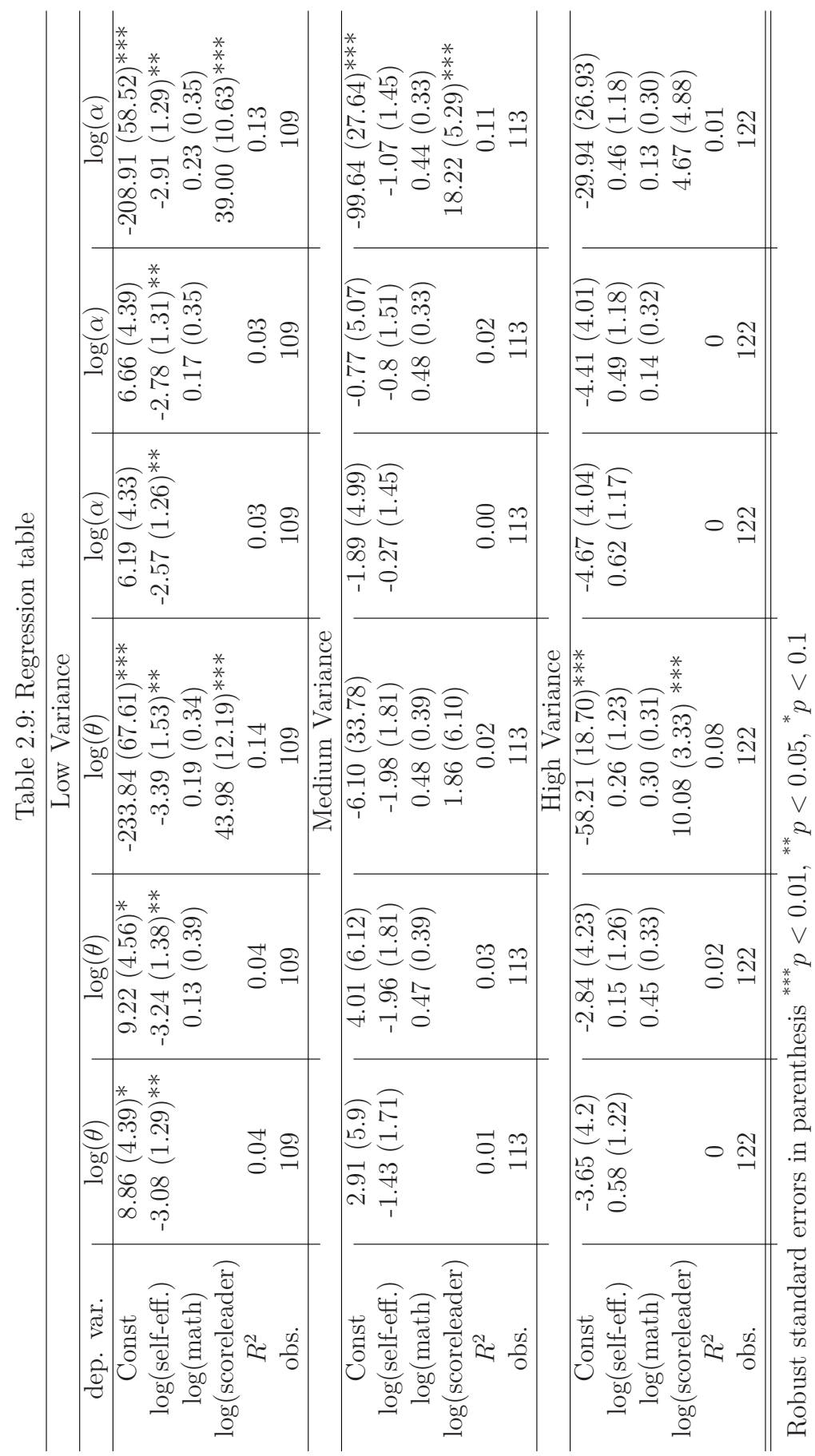


The results of the log-log regressions can be easily interpreted as elasticities. ${ }^{24}$ As it can be seen in the upper part of Table 2.9, a 1\% increase in self-efficacy beliefs corresponds to about a $3 \%$ decrease in the propensity to imitate. This result is stable regardless of the social learning model used. Its significance is not affected when controlling for math abilities or the leader's score. We observe that math skills seem to have no effect, suggesting that subjects were able to cope with the logic implicit in the task. Subjects were also observant enough to identify when leaders were doing well, and gave more weight to observations of leaders whose payoffs were high. Although this confirms the general claim that agents tend to imitate well-performing individuals, this does not reduce the magnitude of the coefficient for self-efficacy. It only strengthens our hypothesis. In easy environments, weakly self-efficacious individuals are more apt at following the leader's action, independently from the leader's score. In the medium and high variance cases instead, we cannot reject the null hypothesis. We do know that higher variance in the payoffs introduces noise in the signals received by the players, thus making the relationship between self-efficacy and propensity to imitate harder to detect. It seems there was simply too much noise in the signals for subjects to use information effectively.

\subsection{Conclusions}

In economics it is often assumed that individuals imitate others for purely instrumental reasons. Agents feel compelled to imitate co-players if they think they have more information or if they are able to obtain higher payoffs. The goal of this study is to go beyond pure cost-benefit analysis calculations and consider that human beings are 'cognitive misers' and might engage in imitative behaviours for non-instrumental reasons, such as low self-efficacy beliefs.

In the laboratory our subjects played a modified version of the two-armed bandit problem with finite time horizon. We retrieved information on their self-efficacy beliefs in order to evaluate the extent to which introspective beliefs concerning one's abilities to understand an control external environments affect the propensity to copy a peer's actions. To assess imitative behaviours correctly, we first studied how individuals play when alone. We modelled the binary choice problem using the standard logit model. We posit

\footnotetext{
${ }^{24}$ The results of lin-lin, $\log$-lin and lin-log specifications can be found in Appendix 2.8.
} 
that as subjects gather experience or information about the options, they update their beliefs according to selected learning models. We then estimate two models of imitation. We do not consider imitation as a substitute to individual learning but rather a possible complement to it. Imitative behaviour is the joint outcome of individual learning and the opportunity to observe a peer.

Our results point to a negative relation between self-efficacy beliefs and imitative behaviours. Regardless of the model used, more self-efficacious agents seem to follow their group leader to a lesser extent. Specifically, a $1 \%$ increase in self-efficacy beliefs corresponds to about a $3 \%$ decrease in the propensity to imitate. This establishes a nexus between individuals' introspective beliefs and the way in which agents learn when given the possibility to observe a peer's actions and outcomes. These effects are strongest in low volatility environments, effectively disappearing when volatility gets too high. Nonetheless, taking into account human cognitive capacities seems to reveal another micro-mechanism able to explain why people imitate others.

We should mention two caveats to these results. The first is that we have taken selfefficacy as fixed over the course of the experiment. It was "measured" at the start of the experiment by a questionnaire, and assumed not to change. But in fact we know that selfefficacy can be changed by experience. Bandura himself claims that whilst self-efficacy affects behaviour and learning, learning processes feedback into self-efficacy perceptions. We have assumed that the experiment was short enough that this feedback had little effect. Second we restricted our analysis to a case in which people observe a peer and possibly imitate his action. We did not consider the effect of social interactions in the form of communication for example. We also did not allow for subjects to select the coplayer to be imitated. Doing so would demand a different, and probably more complex, experimental design, but taking this into account would represent an interesting next step in formulating a model of how people decide to imitate others.

Imitation is sometimes referred to as 'the poor man's rationality' [Offerman and Schotter, 2009]. Offerman and Schotter [2009] argue that in case a decision maker were fully rational and capable of effortlessly carrying out all necessary calculations, he would not feel any need to imitate anyone. Our finding strengthens this statement and sheds some light on the reason why imitation occurs. Given the design of our experiment, the leader is providing a focal point for behaviour. The mere fact that all group members see the 
choice made by the leader is in itself likely to prompt compliance. But we find that, after controlling for their abilities to process simple information and the leader's quality, selfefficacious people are less apt to follow the leader's action, suggesting that self-efficacy acts as a motivator to act independently. This seems to suggest that in communities characterised by low volatility and where the general population has low self-efficacy beliefs, people are more inclined to follow, and to mimic the behaviour of prominent individuals regardless of the goodness of their actions. Low self-efficacy, or more generally a failure to aspire to one's own potential, can lead to unconditional imitation, and to the perpetuation of possibly inefficient behaviours.

In order for institutional change to occur, individuals must perceive that change is possible and that they could achieve better outcomes under a set of alternative rules of the game. But this alone is not enough. People should also believe that they can effectively bring about the change they seek. This might imply departing from standing norms, past behaviour and the behaviour of their neighbours. If people have little faith in their capacities to achieve any designated goal, they will neither see nor pursue any opportunity to better their conditions. As a consequence people will tend to follow the behavioural path set by somebody else, thus conforming to the past and promoting institutional reproduction [Patterson, 2010]. In turn, institutional change depends on the existence of a sub-population that is willing to ignore the behaviour of others and 'strike out on their own'. Whether such a population could exist, will depend, at the individual level, on whether people have beliefs in their ability to effect change, that is to say, their selfefficacy. Those with low self-efficacy beliefs will continue to imitate others, and thereby strengthen the institution, those with high-self-efficacy beliefs might be willing to depart from the others and try to create circumstances which not only better themselves, but might also open the door to institutional change. 


\subsection{Appendix: Monte Carlo Simulations}

True beta $=0.6$, True $r=0.61$

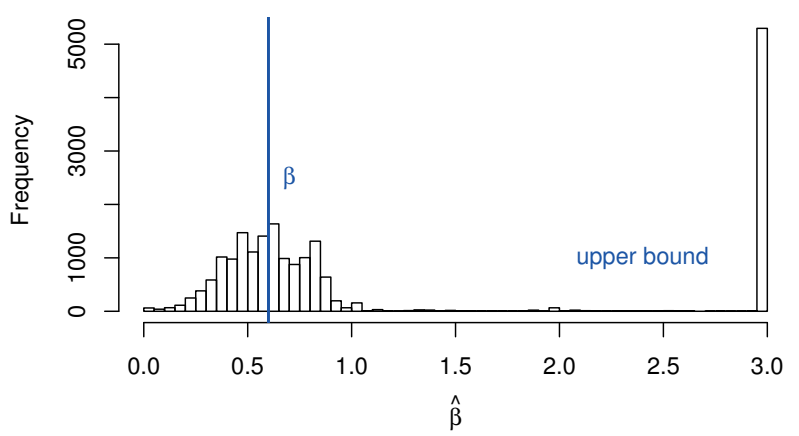

Figure 2.6: Distribution $\hat{\beta}$ : Monte Carlo simulations (Low Variance)

True beta $=0.42$, True $r=0.26$

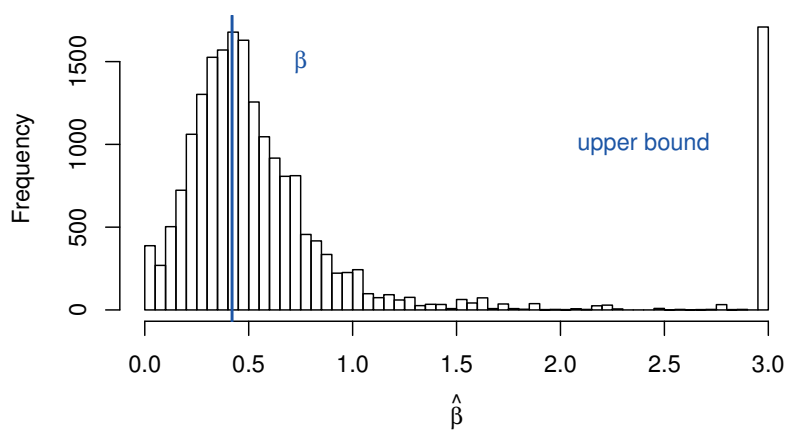

Figure 2.7: Distribution $\hat{\beta}$ : Monte Carlo simulations (Medium Variance)

True beta $=0.28$, True $r=0.40$

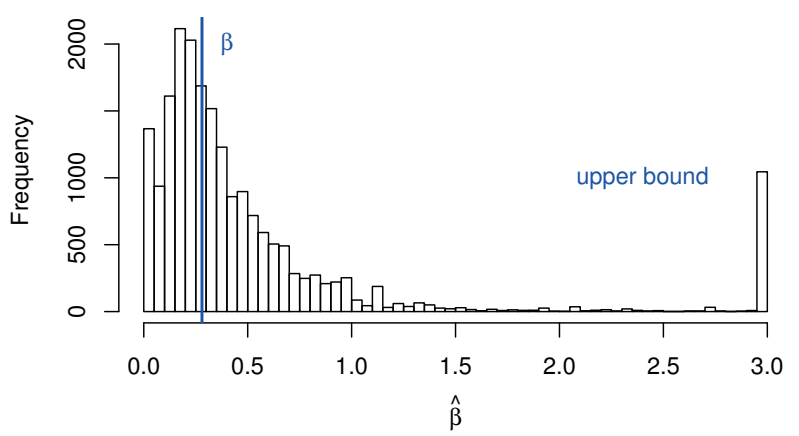

Figure 2.8: Distribution $\hat{\beta}$ : Monte Carlo simulations (High Variance) 


\subsection{Appendix: Regression Tables}

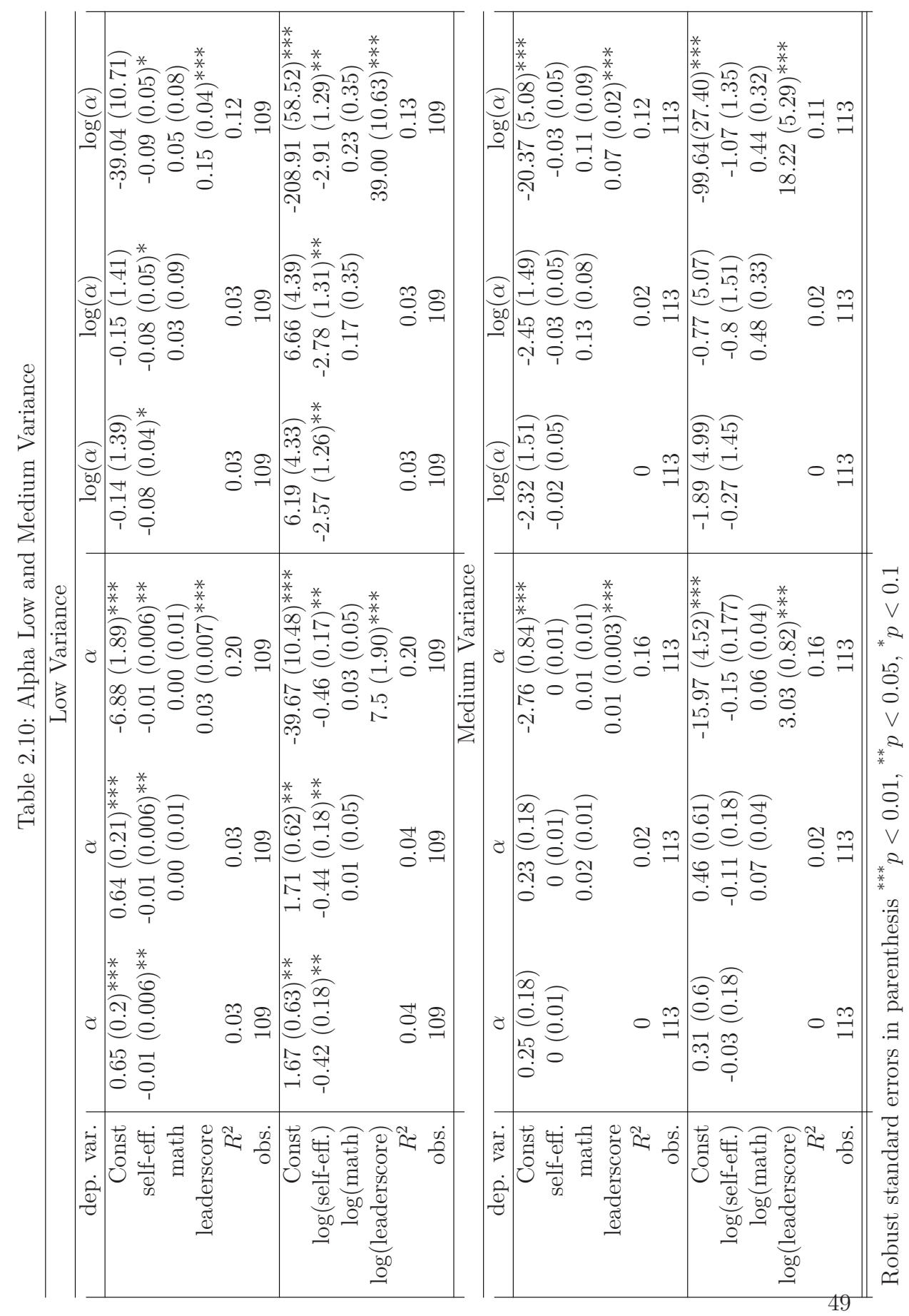




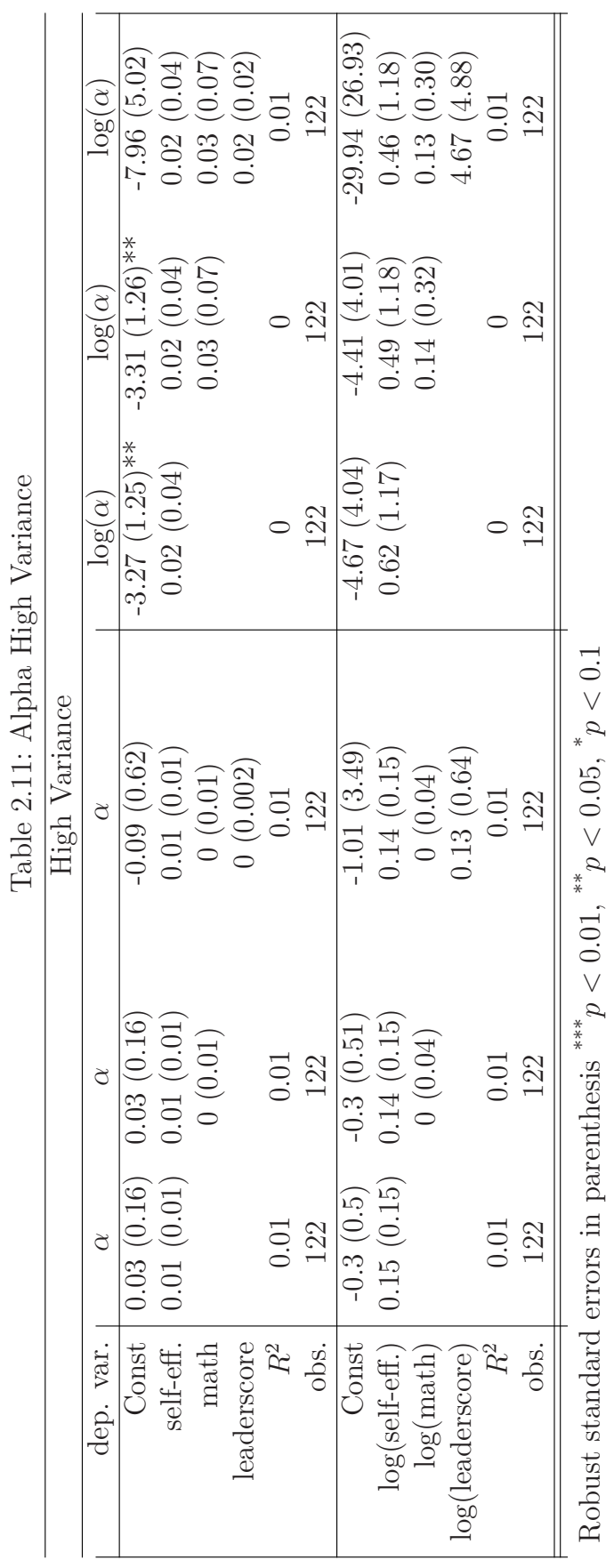




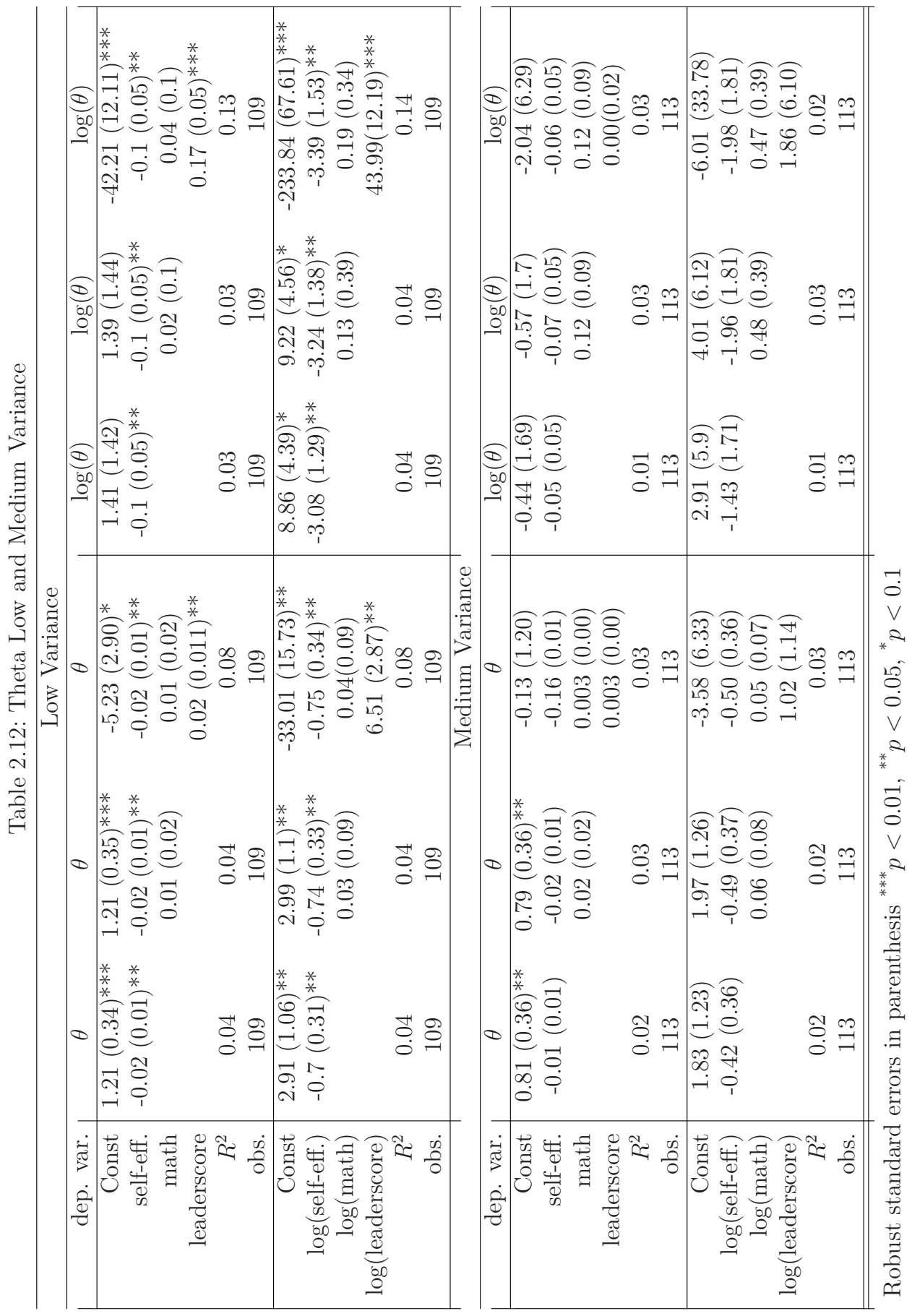




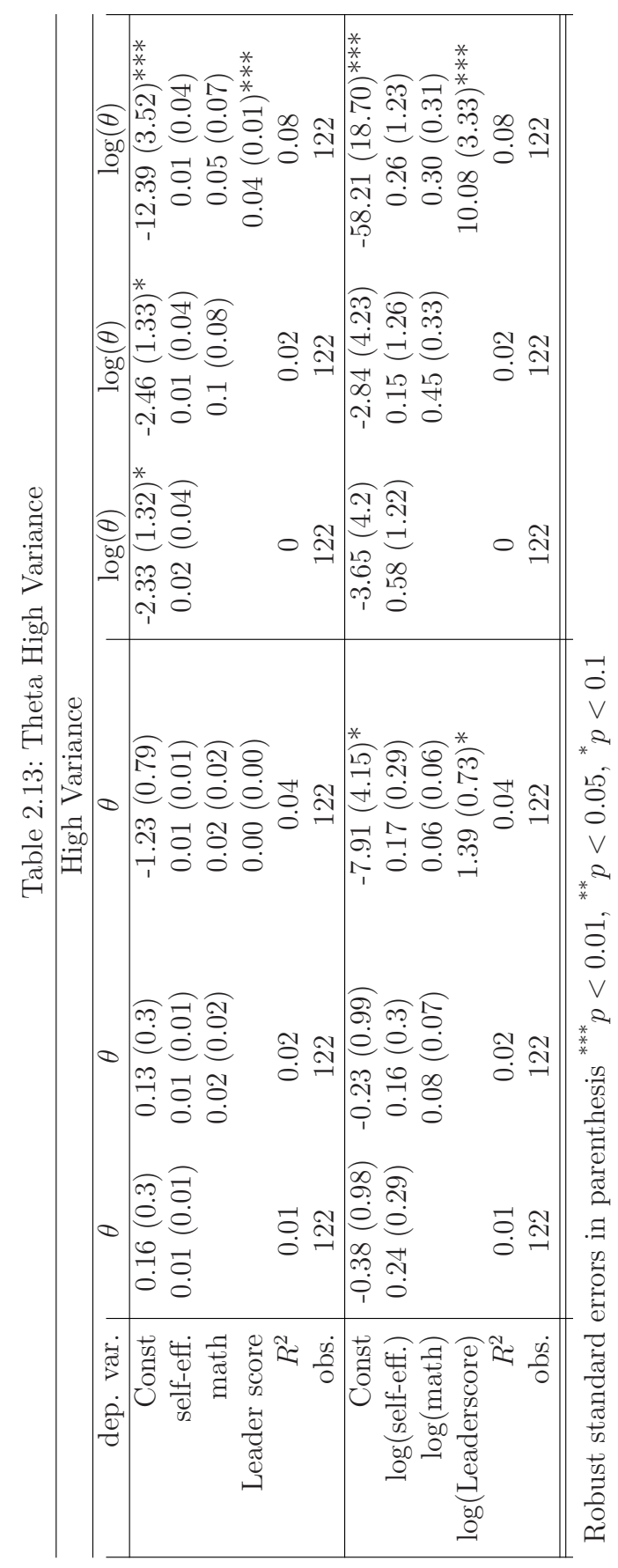




\subsection{Appendix: Experimental Instructions}

This appendix contains all the experimental instructions for our experiment. Specifically, we report screenshots for all parts of the experiment. Payoffs, conversion rates are exactly those our participants experienced. For illustrative purposes we report, for each part, only 2 out of the 20 rounds of each sub-setting. For this reason, the total score reported on page 27 is lower than the actual final payment received by our participants. Please, note that the third part of this experiment (Part 3) is not the object of this paper. We thus omit reporting the related instructions. Before accessing the laboratory, students were reminded of the lab rules concerning phone usage and communication with the experimenter. No communication amongst subjects was allowed. Subjects could opt out of the experiment at all times. 


\section{Manowe Maastricht University}

Welcome to today's experiment.

All instructions for this experiment will appear on your screen. Please, read them carefully. Remember that you are not allowed to use the back button on your web-browser, if you do so you will exit the game. A part of the experiment requires you to wait that other participants have input their answers. Please, be patient and often refresh the page using the link prodided. Moreover, please remember that at any point in time during this game, you are not allowed to use pen and and paper. You are also absolutely forbidden to surf the web.

DO NOT CLICK ON QUESTIONNAIRE I UNTILL THE EXPERIMENTER SAYS SO!

\section{Questionnaire I}

You have 12 minutes to complete this questionnaire. There is only one correct answer for each question. After the 12 minutes the computer will automatically register your missing answers as incorrect.

[WAITING Click here to refresh the page] Questionnaire II

[WAITING Click here to refresh the page] Part 1

[WAITING Click here to refresh the page] Part 2

[WAITING Click here to refresh the page] Part $\mathbf{3}$

[WAITING Click here to refresh the page] Final questionnaire

Select 
Maastricht University

\section{9 \\ MINUTES SECONDS}

\section{QUESTIONNAIRE 1}

(1) What is the average (arithmetic mean) of all the multiples of ten from 10 to 190 inclusive?

$\begin{array}{lllll}\bigcirc_{90} & \bigcirc_{95} & \bigcirc_{100} & \bigcirc_{105} & \bigcirc_{110}\end{array}$

2 If the product of 6 integers is negative, at most how many of the integers can be negative?

$\mathrm{O}_{2} \mathrm{O}_{3} \mathrm{O}_{4} \quad \mathrm{O}_{5} \mathrm{O}_{6}$

3 In a sports club with 30 members, 17 play badminton and 19 play tennis and 2 do not play either. How many members play both badminton and tennis?
$\begin{array}{lllll}\bigcirc_{7} & \bigcirc_{8} & \bigcirc_{9} & \bigcirc_{10} & \bigcirc_{11}\end{array}$

(4) A certain animal in the zoo has consumed 39 kilos of food in six days. If it continues to eat at the same rate, in how many more days will its total consumption be 91 kilos?

$\begin{array}{lllll}\mathrm{O}_{12} & \bigcirc_{11} & \bigcirc_{10} & \bigcirc_{9} & \bigcirc_{8}\end{array}$

(5) In a class of 78 students 41 are taking French, 22 are taking German. Of the students taking French or German, 9 are taking both courses. How many students are not enrolled in either course?

$\begin{array}{lllll}\bigcirc_{6} & \bigcirc_{15} & \bigcirc_{24} & \bigcirc_{33} & \bigcirc_{54}\end{array}$

$63 x+y=19$ and $x+3 y=1$

Find the value of $2 x+2 y$

$\bigcirc_{20} \bigcirc_{18} \bigcirc_{11} \bigcirc_{10} \bigcirc_{5}$

7 Andy solves problems 74 to 125 inclusive in a Math exercise.

How many problems does he solve?

$\bigcirc_{53} \bigcirc_{52} \bigcirc_{51} \bigcirc_{50} \bigcirc_{49}$

86 people meet for a business lunch.

Each person shakes hands once with each other person present

How many handshakes take place?

$\bigcirc_{30} \bigcirc_{21} \bigcirc_{18} \bigcirc_{15} \bigcirc_{10}$

9 A triangle has a perimeter equal to 13

The two shorter sides have integer lengths equal to $x$ and $x+1$ Which of the following could be the length of the other side?

$\mathrm{O}_{4} \mathrm{O}_{6} \quad \mathrm{O}_{8}$

10 Sheila works 8 hours per day on Monday, Wednesday and Friday, and 6 hours per day on Tuesday and Thursday. She does not work on Saturday and Sunday. She earns $\$ 324$ per week.

How much does she earn in dollars per hour?

$\begin{array}{lllll}\bigcirc_{11} & \bigcirc_{10} & \bigcirc_{8} & \bigcirc_{7} & O_{7}\end{array}$ 


\section{Maastricht University}

Thank you.

Questionnaire I is now over. Clicking "Back" you will get back to the menu page and select Questionnaire II.

\section{$\mathbf{U}$ Maastricht University

Welcome to today's experiment.

All instructions for this experiment will appear on your screen. Please, read them carefully. Remember that you are not allowed to use the back button on your web-browser, if you do so you will exit the game. A part of the experiment requires you to wait that other participants have input their answers. Please, be patient and often refresh the page using the link prodided. Moreover, please remember that at any point in time during this game, you are not allowed to use pen and and paper. You are also absolutely forbidden to surf the web.

DO NOT CLICK ON QUESTIONNAIRE I UNTILL THE EXPERIMENTER SAYS SO!

[COMPLETED] Questionnaire I

You have 12 minutes to complete this questionnaire. There is only one correct answer for each question. After the 12 minutes the computer will automatically register your missing answers as incorrect.

\section{OQuestionnaire II}

[WAITING Click here to refresh the page] Part 1

[WAITING Click here to refresh the page] Part 2

[WAITING Click here to refresh the page] Part 3

[WAITING Click here to refresh the page] Final questionnaire

Select

\section{() 2015 United Nations University UNU-MERIT \& Maastricht University}




\section{Dast Maastricht University}

Introduction to questionnaire II

You are soon to begin Questionnare II. Please, read carefully all the statements that will appear on the next page and select the answer that best describes you. When you have finished reading these instructions and are ready to begin, click "Start" below. 


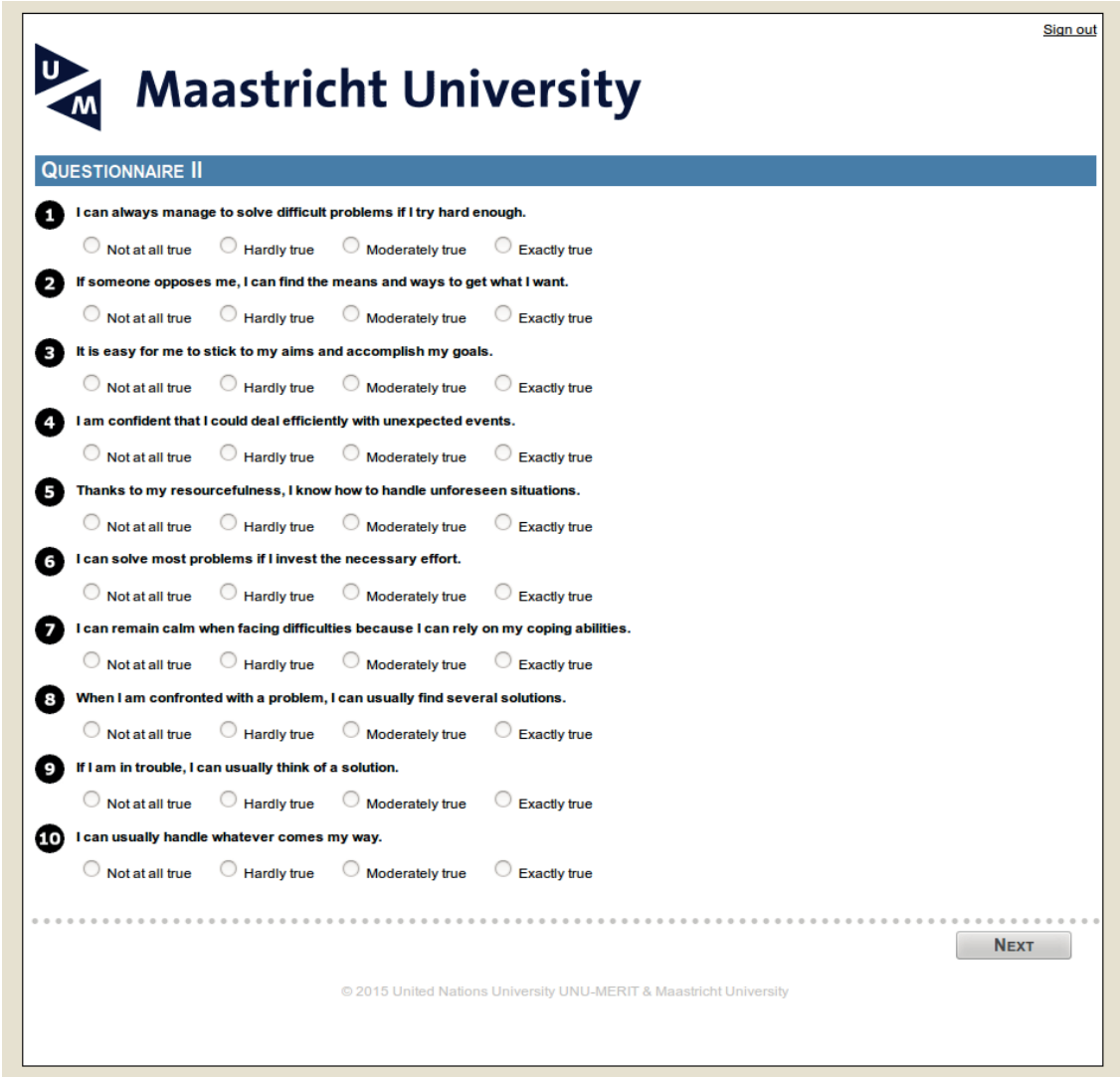




\section{Dastricht University}

Thank you.

Questionnaire II is now completed. You will now get back to the menu page and select Part 1.

\section{Masticht University Maastricht University}

\section{Welcome to today's experiment.}

All instructions for this experiment will appear on your screen. Please, read them carefully. Remember that you are not allowed to use the back button on your web-browser, if you do so you will exit the game. A part of the experiment requires you to wait that other participants have input their answers. Please, be patient and often refresh the page using the link prodided. Moreover, please remember that at any point in time during this game, you are not allowed to use pen and and paper. You are also absolutely forbidden to surf the web.

DO NOT CLICK ON QUESTIONNAIRE I UNTILL THE EXPERIMENTER SAYS SO!

[COMPLETED] Questionnaire I

You have 12 minutes to complete this questionnaire. There is only one correct answer for each question. After the 12 minutes the computer will automatically register your missing answers as incorrect.

[COMPLETED] Questionnaire II

\section{Part 1}

[WAITING Click here to refresh the page] Part 2

[WAITING Click here to refresh the page] Part 3

[WAITING Click here to refresh the page] Final questionnaire

Select 


\section{Maastricht University}

\section{Welcome to the first part of this experiment}

In all 3 sub-settings of this part, you will choose between two colours. Each sub-setting contains 20 rounds and has some peculiar features. This implies that the two colours give different outcomes in each sub-setting. In any single sub-setting, one of the two colours is on average better than the other. Possible scores range from 1 to 18 and may vary from round-to-round and from person-to-person. After every choice you make, you will be informed about the score from that choice in tokens. Every decision affects how much money you will earn today.

After the 20th round of the third sub-setting, this part of the experiment will be over, and you will progress to part 2.

When you have finished reading these instructions and are ready to begin part 1, click "Continue" below. You will automatically start with the first sub-setting of part 1.

\section{Maastricht University}

\section{Game's General instruction}

You are soon to begin to play. This game consists of 3 main parts. Each part contains three consecutive sub-settings of 20 rounds each.

Your task is to choose between two alternative colours.

Once your choice is made, we will inform you about the score from that choice in tokens. 120 tokens will correspond to 1 euro.

At the end of the experiment, the enumerator will pay you for your total reward obtained in all parts. 


\section{Maastricht University}

First Sub-SETting

(1) Black or white?

Black White

Next

Maastricht University

\section{FIRST SUB-SETTING}

In the previous round, you chose "White" and obtained 13 tokens.

2 Black or White?

- Black White 


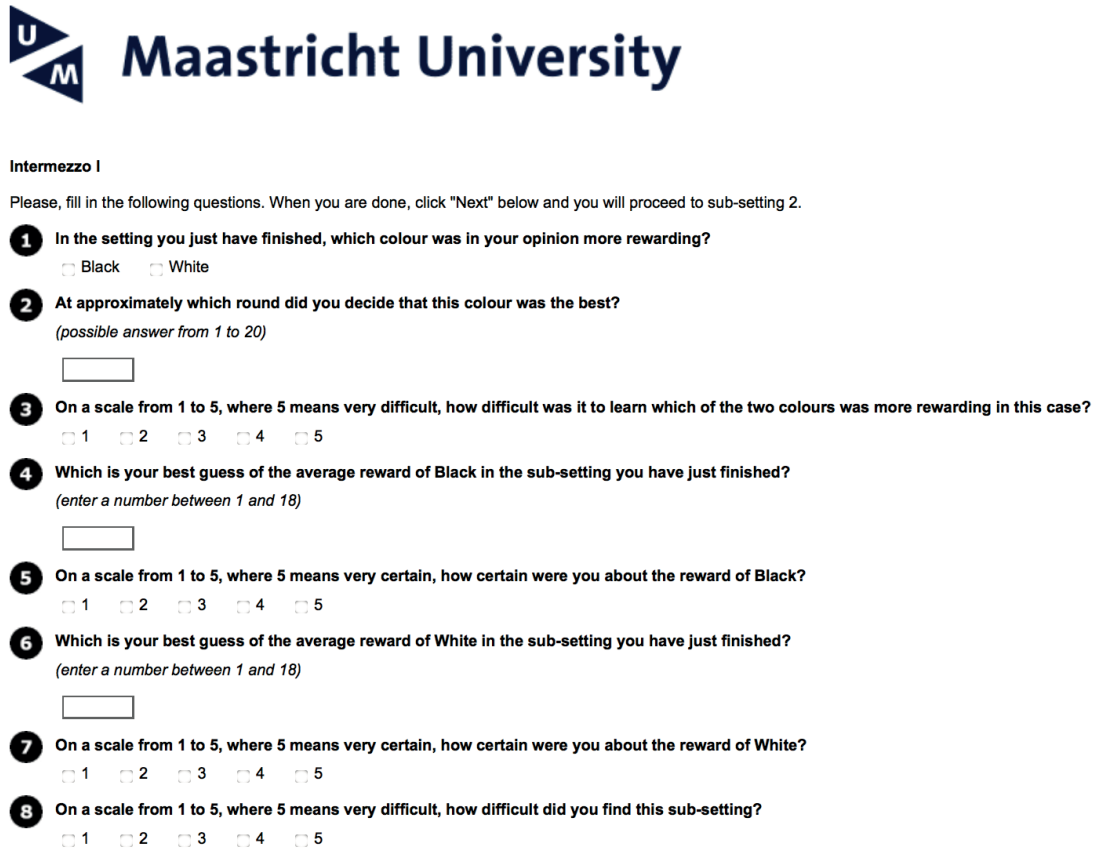

5 On a scale from 1 to 5 , where 5 means very certain, how certain were you about the reward of Black?

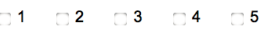

6 Which is your best guess of the average reward of White in the sub-setting you have just finished? (enter a number between 1 and 18)

7 On a scale from 1 to 5 , where 5 means very certain, how certain were you about the reward of White? $\begin{array}{lllll}1 & 2 & 3 & 4\end{array}$

8 On a scale from 1 to 5 , where 5 means very difficult, how difficult did you find this sub-setting? $\begin{array}{lllll}1 & 2 & 3 & 4 & 5\end{array}$

\section{Maastricht University}

Welcome to part one sub-setting two.

You are now facing two new colours. 


\section{Maastricht University}

\section{Second Sub-setting}

(1) Red or Yellow?

Red Yellow

\section{Maastricht University}

\section{Second Sub-setting}

In the previous round, you chose "Yellow" and obtained 13 tokens.

2 Red or Yellow?

- Red $\bigcirc$ Yellow 


\section{Mastricht University}

Intermezzo II

Please, fill in the following questions. When you are done, click "Next" below and you will proceed to sub-setting 3

(1) In the sub-setting you just have finished, which colour was in your opinion more rewarding? Red Yellow

2 At approximately which round did you decide that this colour was the best? (possible answer from 1 to 20 )

3 On a scale from 1 to 5 , where 5 means very difficult, how difficult was it to learn which of the two colours was more rewarding in this case? $\begin{array}{llllll}1 & 2 & 3 & 4\end{array}$

4 Which is your best guess of the average reward of Red in the sub-setting you have just finished? (enter a number between 1 and 18)

5 On a scale from 1 to 5 , where 5 means very certain, how certain were you about the reward of Red? $\begin{array}{lllll}1 & 2 & 3 & 4 & 5\end{array}$

6 Which is your best guess of the average reward of Yellow in the sub-setting you have just finished? (enter a number between 1 and 18)

7 On a scale from 1 to 5 , where 5 means very certain, how certain were you about the reward of Yellow? $\begin{array}{lllll}1 & 2 & 3 & 5\end{array}$

8 On a scale from 1 to 5 , where 5 means very difficult, how difficult did you find this sub-setting?

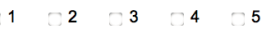

\section{Maastricht University}

Welcome to part one sub-setting three.

You are now facing two new colours. 


\section{Mastricht University}

\section{THIRd Sub-setting}

(1) Green or Blue?

Green Blue

\section{Maastricht University}

\section{THIRd Sub-setTINg}

In the previous round, you chose "Green" and obtained 17 tokens.

2 Green or Blue?

Green Blue 


\section{Maastricht University}

\section{Intermezzo III}

Please, fill in the following questions. When you are done, click "Next" below and you will be informed about the total score you obtained in this part.

1. In the setting you just have finished, which colour was in your opinion more rewarding? Green Blue

2 At approximately which round did you decide that this colour was the best? (possible answer from 1 to 20)

3 On a scale from 1 to 5 , where 5 means very difficult, how difficult was it to learn which of the two colours was more rewarding in this case? $\begin{array}{llllll}1 & 2 & 3 & 4 & 5\end{array}$

4 Which is your best guess of the average reward of Green in the setting you have just finished? (enter a number between 1 and 18)

5 On a scale from 1 to 5 , where 5 means very certain, how certain were you about the reward of Green? $\begin{array}{lllllll}1 & 2 & 3 & 4 & 5\end{array}$

6 Which is your best guess of the average reward of Blue in the setting you have just finished? (enter a number between 1 and 18)

7 On a scale from 1 to 5 , where 5 means very certain, how certain were you about the reward of Blue? $\begin{array}{llllll}1 & 2 & 3 & 4\end{array}$

8 On a scale from 1 to 5 , where 5 means very difficult, how difficult did you find this setting? $\begin{array}{lllllll}1 & 2 & 3 & 4 & 5\end{array}$

\section{Dastricht University}

Your total score for this part was: 110 tokens

Thank you .

Part 1 is over.

Clicking "Back" you will now get back to the menu page and select part 2. 


\section{$\rightarrow$ Mastichtuniversity Maastricht University}

\section{Welcome to today's experiment.}

All instructions for this experiment will appear on your screen. Please, read them carefully. Remember that you are not allowed to use the back button on your web-browser, if you do so you will exit the game. A part of the experiment requires you to wait that other participants have input their answers. Please, be patient and often refresh the page using the link prodided. Moreover, please remember that at any point in time during this game, you are not allowed to use pen and and paper. You are also absolutely forbidden to surf the web.

DO NOT CLICK ON QUESTIONNAIRE I UNTILL THE EXPERIMENTER SAYS SO!

[COMPLETED] Questionnaire I

You have 12 minutes to complete this questionnaire. There is only one correct answer for each question. After the 12 minutes the computer will automatically register your missing answers as incorrect.

[COMPLETED] Questionnaire II

[COMPLETED] Part 1

- Part 2

[WAITING Click here to refresh the page] Part 3

[WAITING Click here to refresh the page] Final questionnaire 


\section{Maastricht University}

\section{General instructions}

\section{Welcome to the second part of this experiment}

As in part 1, in all 3 sub-settings of this part, you will choose between two colours. Each sub-setting contains 20 rounds and has some peculiar features. This implies that the two colours give different outcomes in each sub-setting. In any single sub-setting, one of the two colours is on average better than the other. Possible scores range from 1 to 18 and may vary from round-to-round and from person-to-person. After every choice you make, you will be informed about the score from that choice in tokens. Every decision affects how much money you will earn today.

Differently from part 1, every person in this experiment is now a member of a group of four or five people randomly selected from all current participants. However, none of you will know who the members of your group are. Everyone belonging to the same group will make his or her decisions under exactly the same conditions, so the same colour is best for each person in the group.

Each group will have a leader who will operate under exactly the same conditions as all other group members. The leader has been randomly selected. You will not be able to tell who your leader is. However, you will, in each round of each sub-setting of this part, observe what your group leader has just chosen and the reward he or she obtained from his or her choice.

After the 20th round of the third sub-setting, this part of the experiment will be over, and you will progress to part 3 .

When you have finished reading these instructions and are ready to begin part 2, click "Continue" below. 


\section{Maastricht University}

\section{Welcome to part two sub-setting one.}

As in part one, your task is to choose between two colours. You are now part of a group which is led by a leader. You will not be able to know his or her identity but in each round of this part, you will be able observe what your group leader has just chosen and the reward he or she obtained from his or her choice. Moreover, after every choice you make, you will be informed about the score from that choice in tokens.

If you have been selected as the leader, this will be made explicit to you in the next page.

When you have finished reading these instructions and are ready to begin with sub-setting one, click "Continue" below. 
Leaders' instructions and game

\section{Maastricht University}

\section{First Sub-SETting}

\section{You are the leader of a group of 4 or 5 people.}

You will be the leader for the next 3 sub-settings. You will play before everyone else and you will not be able to observe anyone else's choices. Your group members, will observe your choices and your scores. In any single sub-setting, one of the two colours is on average better than the other. In all sub-settings after every choice you make, you will be informed about the score from that choice in tokens. You will now begin choosing which one of the two colours to select.

1 Orange or Purple?

Orange Purple

Next

\section{Maastricht University}

\section{FIRST SUb-SETTINe}

In the previous round, you chose "Orange" and obtained 12 tokens.

2 Orange or Purple?

Orange Purple 


\section{Dastricht University}

Welcome to part two sub-setting two.

You are now going to choose between two new colours. You are still the leader of your group.

\section{Maastricht University}

Second Sub-Setting

1 Grey or Pink?

Grey Pink 


\section{Maastricht University}

\section{Second Sub-SETting}

In the previous round, you chose "Pink" and obtained 10 tokens.

2 Grey or Pink?

- Grey Pink

\section{Maastricht University}

Welcome to part two sub-setting three.

You are now going to choose between two new colours. You are still the leader of your group. 
Maastricht University

THIRD SUB-SETTING

(1) Brown or Turquoise?

Brown Turquoise

NeXT

Maastricht University

\section{THIRD SUB-SETTING}

In the previous round, you chose "Brown" and obtained 17 tokens.

(2) Brown or Turquoise?

Brown Turquoise 
2 Self-efficacy beliefs and imitation: a two-armed bandit experiment

Non-leaders' instructions and game

Maastricht University

FIRST SUB-SETTING

Your group leader has just chosen "Orange" and he or she obtained 10 tokens.

1 Orange or Purple?

Orange Purple

NEXT

\section{Maastricht University}

\section{FIRST SUB-SETTING}

In the previous round, you chose "Orange" and obtained 10 tokens.

Your group leader has just chosen "Orange" and he or she obtained 12 tokens.

2

Orange or Purple?

Orange Purple 


\section{Maastricht University}

\section{SECOND SUB-SETTING}

Your group leader has just chosen "Grey" and he or she obtained 13 tokens.

(1) Grey or Pink?

Grey Pink

\section{Maastricht University}

\section{SECOND Sub-SETTING}

In the previous round, you chose "Pink" and obtained 10 tokens.

Your group leader has just chosen "Grey" and he or she obtained 13 tokens.

2 Grey or Pink?

$\bigcirc$ Grey Pink 


\section{Maastricht University}

\section{THIRd Sub-SETTING}

Your group leader has just chosen "Turquoise" and he or she obtained 16 tokens.

(1) Brown or Turquoise?

- Brown $\bigcirc$ Turquoise

\section{Maastricht University}

\section{THIRD SUB-SETTING}

In the previous round, you chose "Brown" and obtained 17 tokens.

Your group leader has just chosen "Brown" and he or she obtained 17 tokens.

2 Brown or Turquoise?

Brown Turquoise 
Intermezzo questionnaire for leaders and non-leaders

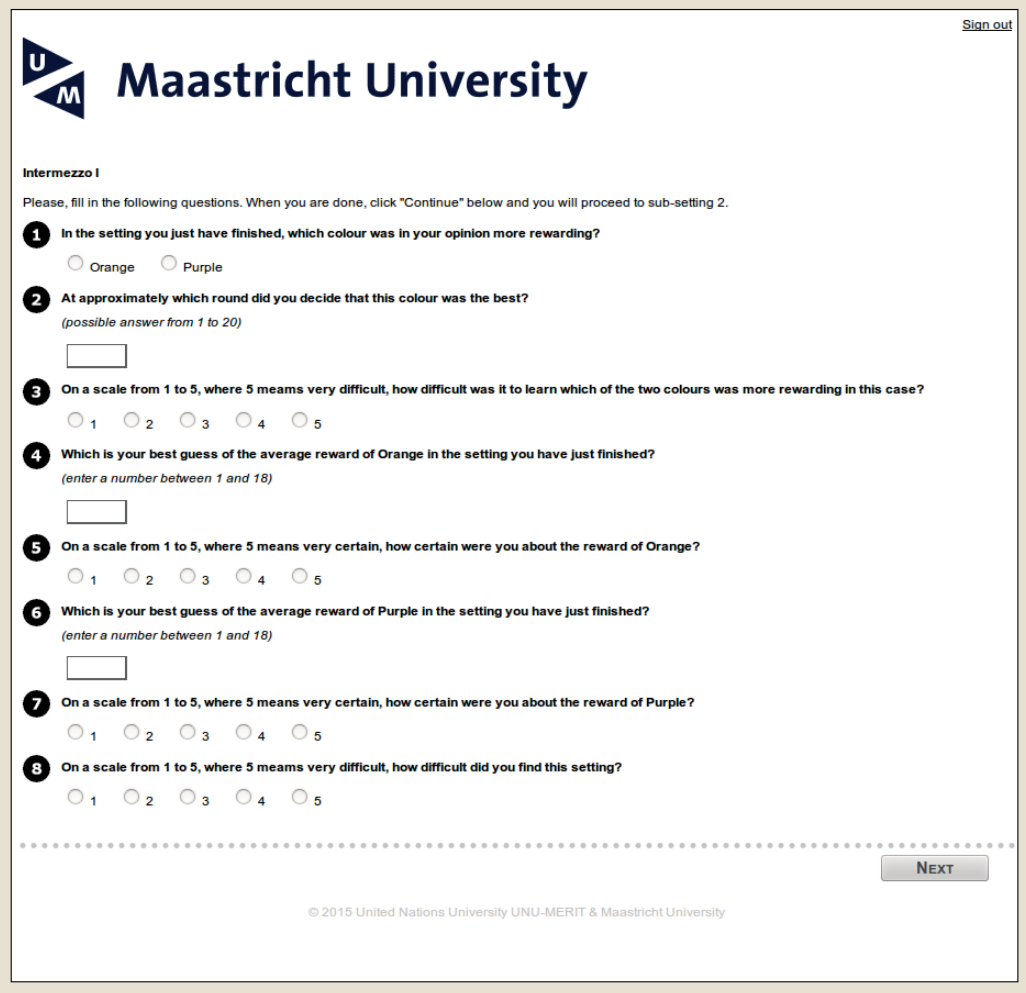




\section{Maastricht University}

Intermezzo II

Please, fill in the following questions. When you are done, click "Continue" below and you will proceed to sub-setting 3 .

(1) In the setting you just have finished, which colour was in your opinion more rewarding?

Grey Pink

2 At approximately which round did you decide that this colour was the best? (possible answer from 1 to 20 )

3 On a scale from 1 to 5 , where 5 means very difficult, how difficult was it to learn which of the two colours was more rewarding in this case? $\begin{array}{lllll}1 & 2 & 3 & 5\end{array}$

4 Which is your best guess of the average reward of Grey in the setting you have just finished? (enter a number between 1 and 18)

5 On a scale from 1 to 5 , where 5 means very certain, how certain were you about the reward of Grey?

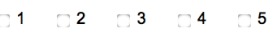

6 Which is your best guess of the average reward of Pink in the setting you have just finished? (enter a number between 1 and 18)

7 On a scale from 1 to 5 , where 5 means very certain, how certain were you about the reward of Pink? $1 \quad 2 \quad 3 \quad 4 \quad 5$

8 On a scale from 1 to 5 , where 5 means very difficult, how difficult did you find this setting? $\begin{array}{lllll}1 & 2 & 3 & 4\end{array}$ 


\section{Maastricht University}

Intermezzo III

Please, fill in the following questions.

When you are done, click "Next" below and you will be informed about the total score you obtained in this part.

(1) In the setting you just have finished, which colour was in your opinion more rewarding? - Brown Turquoise

2 At approximately which round did you decide that this colour was the best? (possible answer from 1 to 20)

3 On a scale from 1 to 5 , where 5 means very difficult, how difficult was it to learn which of the two colours was more rewarding in this case? $\begin{array}{lllll}0 & 2 & 3 & 4 & 5\end{array}$

4 Which is your best guess of the average reward of Brown in the setting you have just finished? (enter a number between 1 and 18)<smiles>C1=CCCC=C1</smiles>

5 On a scale from 1 to 5 , where 5 means very certain, how certain were you about the reward of Brown? $\begin{array}{lllll}0 & 1 & 2 & 3 & 4\end{array}$

6 Which is your best guess of the average reward of Turquoise in the setting you have just finished? (enter a number between 1 and 18)<smiles>C1=CCC=C1</smiles>

7 On a scale from 1 to 5 , where 5 means very certain, how certain were you about the reward of Turquoise? $\begin{array}{lllll}0 & 1 & 2 & 3 & 4\end{array}$

8 On a scale from 1 to 5 , where 5 means very difficult, how difficult did you find this setting? $\begin{array}{llllr}0 & 1 & 2 & 3 & 4\end{array}$

\section{Maastricht University}

Your total score for this part was: 114 tokens

Thank you .

Part 2 is over.

Clicking "Back" you will now get back to the menu page and select part 3. 


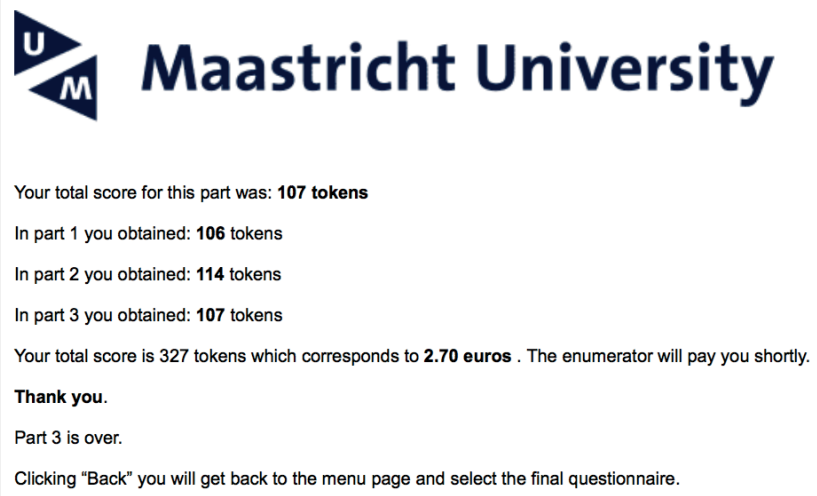




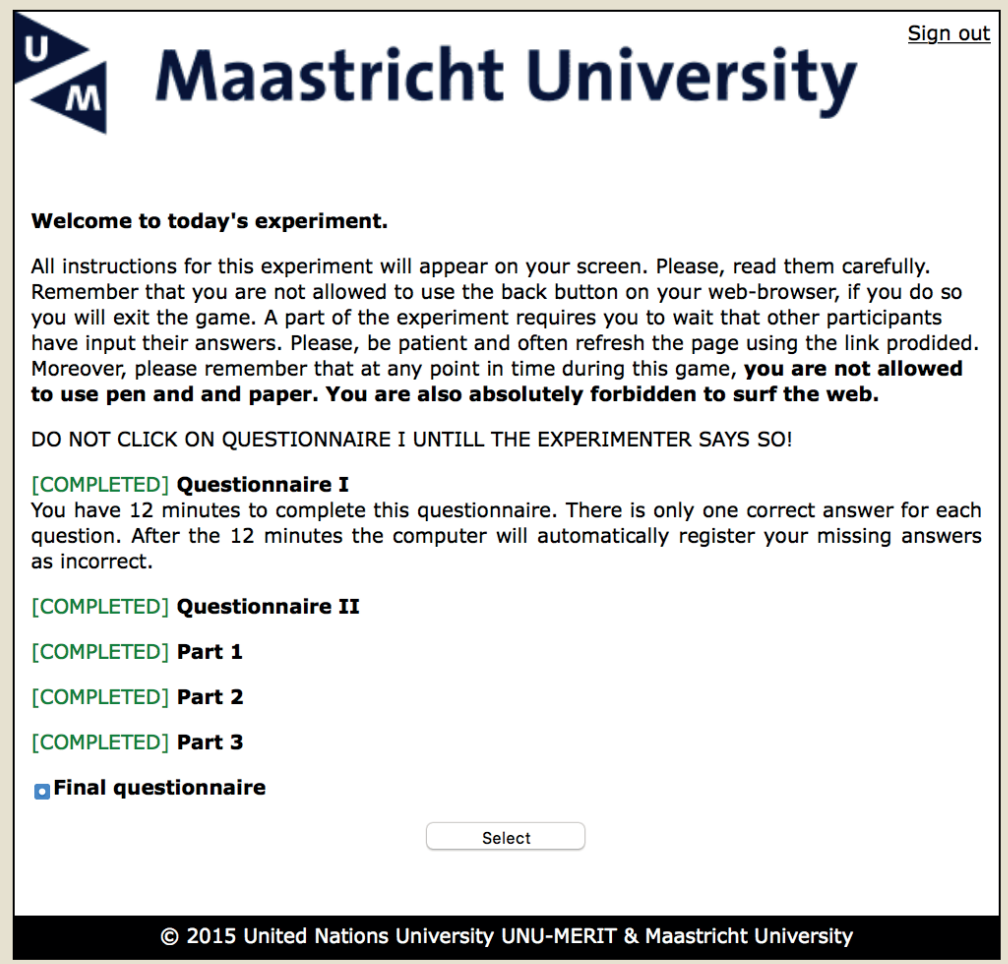


Final questionnaire for leaders

\section{Dastricht University}

Final questionnaire

Today's experiment is nearly over. Please, answer the last questions.

\section{Maastricht University}

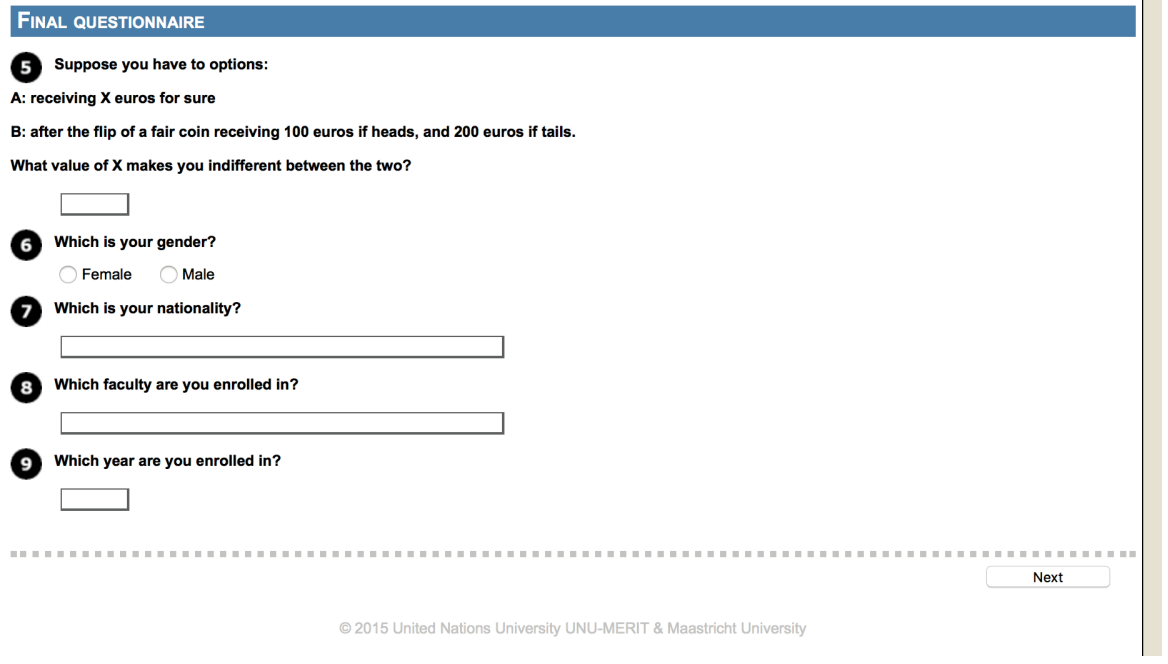


Final questionnaire for non-leaders

\section{In Maastricht University}

Final questionnaire

Today's experiment is nearly over. Please, answer the last questions.

\section{Maastricht University}

\section{FINAL QUeSTIONNAire}

(1) On a scale from 1 to 5 , where 5 means very helpful, how helpful were your leader's decisions to you?

2 On a scale from 1 to 5 , where 5 means very often, how often did you choose what your leader had most recently chosen?

$\begin{array}{lllll}1 & 2 & 3 & 4 & 5\end{array}$

3 On a scale from 1 to 5 , where 5 means very helpful, how helpful were your team members' decisions to you?

4 On a scale from 1 to 5 , where 5 means very often, how often did you choose what the majority of your team members had most recently chosen?

$\begin{array}{llllll}1 & 2 & 3 & 4 & 5\end{array}$

(5) Suppose you have to options:

A: receiving $X$ euros for sure

B: after the flip of a fair coin receiving 100 euros if heads, and 200 euros if tails.

What value of $\mathrm{X}$ makes you indifferent between the two?

6 Which is your gender?

(7) Which is your nationality?

(8) Which faculty are you enrolled in?

9 Which year are you enrolled in? 
2 Self-efficacy beliefs and imitation: a two-armed bandit experiment

\section{Maastricht University}

Thank you

Thanks for participating to this experiment today.

Please, kindly wait in front of your computer untill the enumerator comes and pays you 2.70 euros. 


\title{
CHAPTER 3
}

\section{How do institutions change? Self-efficacy Beliefs and Social Interactions ${ }^{1}$}

\begin{abstract}
Institutional resistance to change is usually considered the result of increasing returns to conformity. I posit that institutional inertia emerges also because most members of the population do not believe in their abilities to change the status quo and thus unquestionably follow the standing behavioural rule. I propose a simple discrete choice model with social interactions and self-reinforcing dynamics. Agents, under the influence of social interactions, asynchronously choose between two alternatives. Over time they learn about their abilities to reach higher individual welfare. Successful individuals are more likely to revise their choice. I first show that, in line with previous literature, high pressure for conformity triggers multiple equilibria. Moreover I show that, with low returns to conformity, when only few pro-active individuals defy the status quo, social innovation is less likely to occur.
\end{abstract}

${ }^{1}$ The author is grateful to Robin Cowan, Gian Italo Bischi, François Lafond, Daniel Opolot, Marco Pangallo and William Zame for their valuable comments and suggestions. 


\subsection{Introduction}

Institutions coordinate people's behaviour. Money facilitates economic transactions. Languages help communication. Property rights define the limits of private property. These are a few among many others institutions which help us coordinate our actions. They emerge as a consequence of individual experimentation and mistakes [Young, 2001, 2015]. Nevertheless, sometimes, institutions support behaviours that are inferior [Belloc and Bowles, 2013]. Thus, in order to allow people to live better lives and develop their potential, institutions should change. Yet, sometimes this does not easily happen endogenously.

What accounts for the persistence of inferior institutions? Or differently, what are the obstacles for institutional innovation to occur endogenously, even if this is socially desirable?

The most widely held explanation in the literature is that institutional inertia is the result of increasing returns to institutional conformity or strategic complementarities. In fact very often a rule of behaviour appears more attractive the higher the number of its adopters [Young, 1998b]. The positive externalities in adoption decisions emerge from advantages of shared behavioural expectations and thus a convergence of beliefs. These factors add to the intrinsic utility one derives from adopting a certain behaviour and are often referred to as "network externalities" or "pressures for conformity".

Using an evolutionary computational framework, I propose a complementary explanation. I posit that institutional inertia emerges not only as a consequence of increasing returns to conformity but also because most members of the population do not believe in their abilities to change the status quo and thus unquestionably follow the standing behavioural rule.

Over the course of their lives, individuals develop beliefs concerning their own abilities to organise their effort and achieve their desired goals. In the psychology literature, such beliefs are usually referred to as self-efficacy [Bandura, 2001], whereas within the economics literature they are generally known as self-confidence. ${ }^{2}$ Self-efficacy increases, if previously undertaken actions result in the outcomes one expects. If instead actions

\footnotetext{
${ }^{2}$ According to Bandura [1997] confidence and self-efficacy are not necessarily the same thing. While selfefficacy refers to the judgement about one's own ability to follow a desired course of action and achieve a specific goal, confidence refers to a one's trust on a wider variety of personal strengths. Self-efficacy responds to the question "Can I achieve what I decide to accomplish?", whereas confidence responds to the question "Do I feel confident about myself?". Nonetheless, the two terms are colloquially often used interchangeably as they both manifest themselves in pro-active behaviours. Economists have mostly focused their attention on confidence and overconfidence more specifically. Overconfidence is defined as the overestimation of one's ability, performance and likelihood of success. Overconfidence has been found to be the explanation for high and persistent entrepreneurial engagement, and high rates of corporate merger and acquisition, despite the high frequency of failures [Camerer and Lovallo, 1999, Malmendier and Tate, 2005]. Barber and Odean [1999] found that overconfidence generates excessive
} 


\subsection{Introduction}

generate unwanted consequences, self-efficacy decreases. As a result, experience-based learning leads to heterogeneous confidence levels across the population members. Not all members of the population are equally likely to decide to defy the current institutional rule and try out a new behaviour. Only those individuals who think they are able to master the institutional choice and increase their individual welfare, try to do so. These individuals are called trendsetters [Bicchieri, 2017]. By contrast, the rest of the population sticks with the standing practice and conforms to the behaviour it prescribes.

This paper shows that institutional inertia is the emergent outcome of individual motivated beliefs and social interactions. Its contribution is threefold. First, I contribute to the growing literature on motivated beliefs [Bénabou, 2015, Bénabou and Tirole, 2016]. Motivated beliefs are self-views. They are those beliefs that individuals hold concerning their own abilities, mental processes and future fate [Bénabou, 2015, Bénabou and Tirole, 2016]. I suggest that people's decision to adhere to or deviate from an established behavioural rule depends on their estimation of their abilities, i.e. self-efficacy. Second, combining network externalities and subjective beliefs allows me to contribute to the literature on endogenous institutional change. Denzau and North [1994] affirmed that the evolution of institutions is tightly intertwined with the dynamics of individual beliefs and expectations. Nonetheless, few scholars have studied whether, together, individual confidence levels and increasing returns to conformity increase the likelihood of institutional persistence. Third, this paper contributes to the literature concerned with social interaction models introducing a methodological innovation. I build upon the well-established framework of Brock and Durlauf [2001a]. I propose a simple discrete choice model with social interactions and self-reinforcing dynamics.

I model a population of $N$ agents each connected to other agents. I frame the institutional change as a competition between an old and a new behavioural rule. Agents can choose between option $\mathrm{B}$, the status quo behaviour, and option $\mathrm{A}$, the innovative one. The utility each agent obtains from the options depends on the intrinsic value of each alternative as well as on the choices made by others. In each period one agent is given the opportunity to revise his choice, and he does so based on a standard discrete choice model. The probability he will best respond depends on a stochastic exogenous parameter. The probability that the agent revises his decision depends positively on how much faith he has in his abilities, i.e. on his self-confidence or self-efficacy perception. Self-efficacy is endogenous, and changes depending on the success of one's past revision attempts. The reinforcement in self-efficacy and its effect on the probability that agents revise their state, represents the main methodological innovation of this paper.

stock market trading. In this paper, I will mostly rely on the concept of self-efficacy. Nonetheless, with some degree of approximation, I will use in some occasions confidence as synonym for self-efficacy. 
The analysis compares the case in which all population members have equal chances to enact the change to the case in which only few individuals seize the available opportunities and assesses whether the former affects the diffusion of the institutional innovation. In line with previous literature I confirm that high pressure for conformity triggers multiple equilibria: societies possibly end up either adopting the new institution or get trapped into the status quo. But in case only more self-efficacious agents try to enact change, such bifurcation occurs when the probability of making mistakes is lower. Additionally I find that, for low returns to conformity, when highly confident individuals seize the opportunity to switch between alternatives, there exists higher resistance to change and lower collective welfare. The majority of the population sticks to the status quo behaviour and the few trendsetters are not able to accomplish a complete behavioural shift.

The rest of the paper is organised as follows. First, in section 3.2, I review the existing literature identifying the interactions amongst the concepts at stake. Second, in section 3.3, I describe the model. Third, I discuss the results in section 3.4. Lastly, section 3.5 concludes outlining possible future extentions of the model.

\subsection{Related literature}

Two elements are key in the process of institutional formation and change: social structures and agency.

Institutions emerge from the interactions amongst agents. They can be thought of as a kind of 'grammar' for social interactions as they define what is acceptable or not within a society or a group [Axelrod, 1986, Bicchieri, 2005]. Analogously to a grammar, institutions are not necessarily the result of human planning. They mostly emerge from individual and collective experimentation and adaption [Young, 2015]. They also exhibit a self-reinforcing dynamics. People, in fact, adhere to certain norms of behaviour if these norms make them better off, or meet their needs, but also if they expect others to adhere as well. Therefore, institutions emerge as a result of social interactions.

At the same time, the evolution of institutions is constrained by the set of ongoing relations among individuals [Hodgson, 2007], i.e. social structures. Social structures, in fact, determine the degree of behavioural interdependence. The (dis-)utility of (not) conforming to a certain rule of behaviour depends on the decisions made by members of one's reference network. Reference networks are defined as the set of people whose judgements and expectations matter when making a particular decision [Bicchieri, 2017].

In order for endogenous institutional change to occur, the old rule of behaviour has to be disputed, and a new rule has to be tried out. This is difficult because individuals often expect to be punished for the abandonment or deviation from the norm. Nonetheless, some 
individuals are more prone than others to experiment and initiate the process of change. These individuals are often referred to as trendsetters i.e. individuals who challenge the convention, try out the new behaviour and possibly catalyse the change. Although the identity of the trendsetters depends on the norm under scrutiny, it is acknowledged that they are endowed with high levels of agency and more specifically high self-efficacy [Bicchieri, 2017].

Agency is defined in sociology as an agent's ability to make decisions and enact them in the world. Along the same lines, social cognitive theory claims that human agency enables individuals to act with the precise goal of exerting control over the quality and nature of their lives [Bandura, 2001]. Agency allows for reaction and adaptation to evolving situations, but it also leads to the creation of more conducive environments for development. The most central and pervasive mechanism affecting human agency is self-efficacy [Bandura, 1989, 2001].

Self-efficacy has been defined as the "people's judgments of their capabilities to organise and execute courses of action required to attain designated types of performances" [Bandura, 1986, p.391]. It is the belief that one can attain the desired results and forestall detrimental ones by means of one's actions [Bandura, 2001]. Differently said, self-efficacy identifies the level of confidence one has in one's own abilities. Self-efficacy generally stems from the continuous integration of the results of previously undertaken actions. Repeatedly successful behaviours might trigger strong efficacy, contrarily reduced self-efficacy perceptions can be the result of failures. Trendsetters, to be willing to deviate from an established institutionalised behaviour and deal with counter-normative actions should have high self-efficacy [Bicchieri, 2017].

The economics literature has mostly focused its attention on self-confidence. Only recently [Bénabou and Tirole, 2016] have introduced self-efficacy - together with other self-views such as self-control, willpower [Bénabou and Tirole, 2004], self-disappointment [Bénabou and Tirole, 2002] - in the economics domain. They consider self-efficacy as a key self-evaluation able to affect motivation and increase the intention to perform certain behaviours. Although, [Bénabou and Tirole, 2016] underline the importance of the goaldirected nature of individual behaviour which depends specifically on self-efficacy levels, their discussion makes strong use of the concept of self-confidence.

To summarise, a closely intertwined relation between social structures, human agency, self-efficacy and institutions exists. And, in order to study endogenous institutional change such relations have to be taken into account. ${ }^{3}$ I use a model of stochastic learning

\footnotetext{
${ }^{3}$ I indeed acknowledge that the institutional dynamics can be manipulated by lawmakers. For instance, in September 1967, traffic in Sweden switched from driving on the left-hand side of the road to the right in order to conform with the neighbouring countries. Swedish citizens were nonetheless against this. In 1955, in a national referendum 80 percent of the Swedish population opposed the driving
} 
dynamics with social interactions. I specifically build upon the Brock and Durlauf's model [2001a] connecting discrete choices with interaction dynamics and self-reinforcement. This framework has been exploited by a number of authors including among others Blume [1995], Brock and Hommes [1997], Durlauf [1993]. It has been used to explain several social phenomena as for instance theory choices in science [Brock and Durlauf, 1999], job searches [Ioannides and Datcher Loury, 2004], tax evasion [Fortin et al., 2007] and technology choices [Zeppini, 2015]. ${ }^{4}$ Yet, it has not been used to model institutional change or the lack thereof. I maintain that this framework fits the purpose. It allows to model institutions while simultaneously considering the role of human agency and social interactions.

Methodologically, the main innovation of my model consists in introducing a selfreinforcing mechanism able to discriminate, on the basis of self-efficacy, which agents have the opportunity to revise their behaviour. To do so, I assume that the revision of one's choice occurs in an asynchronous fashion. This modelling choice makes my model different from Zeppini's [2015] and Brock and Hommes's [1997] as well as from Bénabou's [2015] and Bénabou and Tirole's [2016]. Zeppini [2015] and Brock and Hommes [1997] model synchronous updating. Nonetheless, this might not be a good representation of how institutional change occurs. In fact, according to Streeck and Thelen [2005], institutional change is incremental. It results from the accumulation, over a long period of time, of subtle individual changes. In evolutionary theories of change, institutional mutations are the results of an individual and decentralised selection process. As a consequence, some successful rules of behaviour diffuse within the population, while unsuccessful ones die out [Kingston and Caballero, 2009]. This identifies a gradual transformation which is better represented by an asynchronous updating rule rather than a synchronous one. Bénabou [2015] and Bénabou and Tirole [2016] do not use a binary choice framework and assume instead that self-efficacy directly enters the utility function. I consider self-efficacy as external to the payoff function and only determining the probability an agent updates his choice. This is in line with Bandura's [2001] theory in which self-efficacy is key in determining whether or not individuals engage in a certain behaviour, rather than affecting the payoff one retrieves from it. "People take action when they hold efficacy beliefs [...] that make the effort seem worthwhile. They expect given actions to produce desired outcomes and believe that they can perform those actions" [Bandura, 1997, p. 24].

change. The institutional change eventually occurred as a consequence of a top-down decision of the Swedish parliament. These types of situations are not uncommon but they are not the focus of this paper.

${ }^{4}$ Zeppini's [2015] model follows the Brock and Durlauf [2001a] model but partially departs from it in that Zeppini does not model the expectations of the dynamic variables. Agents do not choose among different predictors but rather between two options which have a different intrinsic profitability. Individuals decides on the basis of their past experience, namely given their prior knowledge concerning the diffusion of the two technologies. 
The next section explains the model in detail.

\subsection{The model}

Consider a population of $N$ agents, members of a full network. The set of actions available to each player is $X, X=\{A, B\}$. Each agent can choose between $\mathrm{A}$ and $\mathrm{B}$. Option B is the status quo behaviour whereas A is the innovative behaviour. Time periods are discrete and denoted by $t=1,2,3, \ldots, T$. The system starts with a share $x$ of the population choosing $\mathrm{B}$ and the rest adopting $\mathrm{A}$. The payoff agent $i$ receives in any given period $t$ from option $A$ or $B$ is conditional on the choices made by all other members of the population and on the intrinsic utility that one derives from the two available options. Therefore the payoffs to $i$ are:

$$
\begin{gathered}
u_{t}(B)=\lambda^{B}+\rho x_{t-1} \\
u_{t}(A)=\lambda^{A}+\rho\left(1-x_{t-1}\right)
\end{gathered}
$$

where $\lambda^{A}$ and $\lambda^{B}$ represent the inherent values of adopting institution $\mathrm{A}$ and $\mathrm{B}$ respectively. I assume that $\lambda^{A}$ and $\lambda^{B}$ are identical across players and constant over time, i.e. there is not institutional progress. But I also assume that one institution is always intrinsically more profitable than the other (i.e. $\lambda=\lambda^{A}-\lambda^{B} \neq 0$ ).

Moreover, the payoffs depend on the share of the population that chooses $A$ or $B$. This is a perfectly observable information. $x_{t-1}$ denotes the share of individuals who chose $\mathrm{B}$ at $t-1$. By the same token, $1-x_{t-1}$ is the proportion of population members who chose option A.

The parameter $\rho$ represents the intensity of positive externalities in agent's decision or differently a measure of the disutility of non-conformance [Blume and Durlauf, 2003]. ${ }^{5}$ It is greater than 0 and is assumed to be same for both options. The quantity $\rho x_{t-1}$ ( or $\left.\rho\left(1-x_{t-1}\right)\right)$ represents thus the self-reinforcing effect of decision externalities.

I assume that individuals, when deciding whether to switch or stick to the status quo behaviour, use a specific perturbed best response model, i.e the log-linear response model [Blume and Durlauf, 2003, 2001, Blume, 1995, Brock and Durlauf, 2001a, Young, 1998b, 2011]. ${ }^{6}$ This means that, when given the chance to revise, agent $i$ will choose option $\mathrm{B}$ with probability

\footnotetext{
${ }^{5}$ Externalities do not have to be positive. In some instances, social interactions could generate negative feedback, as in the case of conspicuous consumption aimed at an increase in status [Zeppini, 2015]. Nonetheless, these cases do not represent the focus of this paper.

${ }^{6}$ This model assumes that each individual experiences a random utility $\tilde{u}_{i, t}=u_{i, t}+\epsilon_{i, t}$ where $\epsilon_{i, t}$, the noise, is iid across agents and known. It has been proved that if $N$ is large and $\epsilon_{i, t}$ follows a double exponential distribution, the probability of choosing the more rewarding option converges to the one
} 


$$
\operatorname{Pr}\left(x_{i, t}=B\right)=\frac{e^{\beta u_{t}(B)}}{e^{\beta u_{t}(A)}+e^{\beta u_{t}(B)}}=\frac{1}{1+e^{\beta \Delta u_{t}}}
$$

The log probability of choosing B minus the log probability of choosing A corresponds to $\beta$ times the payoff difference between the two options (i.e. $\Delta u_{t}=u_{t}(A)-u_{t}(B)$ ). Or differently, according to this model the probability of deviating from the best response declines when the loss in utility increases. In the limit, if $\beta$ is 0 , the probability associated with each choice is 0.5 . When $\beta$ tends to infinity, agents select the best available option with probability close to 1 . This captures the degree of randomness with which the population of agents responds to the benefits associated with the two options. The parameter $\beta$ is generically defined as intensity of choice parameter [Brock and Hommes, 1997] but has also been interpreted as a measure of irrationality [Belloc and Bowles, 2013], inattention [Matêjka and McKay, 2015] or implementation costs [Fudenberg et al., 2015]. We consider $\beta$ as exogenously given, time invariant and homogeneous across population's members. ${ }^{7}$

In this model knowing the share of the population opting for $\mathrm{B}, x_{B} \equiv x$, is enough to know the state of the system in a given period. As one institution is always intrinsically better than the other, the difference in utility between the two alternatives, using Equations 3.1 and 3.2, is:

$$
\Delta u_{t}=\lambda^{A}+\rho\left(1-x_{t-1}\right)-\lambda^{B}-\rho x_{t-1}=\lambda+\rho\left(1-2 x_{t-1}\right)
$$

As mentioned above, in line with the institutional economics literature, I treat change as the result of individual deviations. Thus, I assume that players update their choices asynchronously. Every time period, an agent has the chance to revise his strategy. Which individual has the chance to adjust his behaviour is determined probabilistically. The probability that an agent updates this period is proportional to his self-efficacy level $\left(\operatorname{Pr}(i\right.$, chosen $\left.)=\frac{s_{i, t}}{\sum_{j=1}^{N} s_{j, t}}\right)$. This implies that agents who have higher self-efficacy relative to the entire population are more likely to re-consider their state. This follows Bandura's [p. 512] [1997] argument according to which highly self-efficacious people "refuse to have their lives dictated by detrimental institutional practices". When confronted with a powerless collective, they mobilise their effort to overcome any obstacle and achieve the change they seek. Self-efficacious people self-select to enact change. Moreover, one of the well-established features of self-confidence is the "better-than-average" effect: when estimating their abilities to perform a task, people judge, and possibly overstate, their

obtained by a multinomial logit. In fact, $\beta$ is inversely related to the variance of the noise distribution [Brock and Durlauf, 2001b].

${ }^{7}$ Bao [2012] analysing experimental data finds little heterogeneity in the estimated intensity of choice parameter. And, there is no clear evidence that experience affects $\beta$. 
acumen with the respect to the group average [Alicke et al., 1995, Svenson, 1981]. Both arguments point to the fact that individuals tend to evaluate their abilities with respect to others. Self-efficacious individuals are engaged, pro-active, independent and they stand out from the crowd.

Once the decision on whether to switch to the alternative option or stick to the previously adopted one is made, the agent's self-efficacy is revised. As postulated by Bandura [2001], I consider that self-efficacy never takes negative values. Moreover, self-efficacy varies endogenously as a result of an experience-based learning process. It increases, if previously undertaken actions result into the outcomes one expects. If instead actions generate unwanted consequences, self-efficacy decreases. Thus, self-efficacy levels change in line with the changes in individual utility. To formalise these conditions, I chose the following functional form to model self-efficacy dynamics

$$
\begin{aligned}
\tilde{s}_{i, t} & =s_{i, t-1} \cdot e^{\alpha\left(u_{i, t}-u_{i, t-1}\right)} \\
s_{i, t} & =\frac{\tilde{s}_{i, t}}{\sum_{j=1 \neq i}^{N} s_{j, t}+\tilde{s}_{i, t}}
\end{aligned}
$$

The parameter $\alpha$ is the key parameter of this model. It indicates the intensity of the revision process. In case $\alpha=0$, past successes or failures are not taken into account and self-efficacy does not vary over time, thus everybody is equally likely to revise his or her state. This is the baseline scenario commonly used in the literature. Throughout the analysis, I will compare this simple case with the case in which $\alpha>0$. When $\alpha>$ 0 , previous successes and failures become relevant and self-efficacy changes accordingly. Some agents are thus more likely than others to update their choices.

Section 3.4 reports the results obtained from my analysis.

\subsection{Results}

The analysis unfolds as follows. First, I focus on the case in which everybody is equally likely to revise one's state, i.e when $\alpha=0$. I compare the synchronous and asynchronous updating rules showing analytically that the two rules lead to the same set of steady states. As a second step, I concentrate on the case in which more self-efficacious agents are more likely to update their choices (i.e. when $\alpha>0$ ) and the updating occurs asynchronously. I derive the new map and corresponding steady states. Subsequently, by means of simulations, I study the system's long-run dynamics. I compare the simulations' results and theoretical predictions and evaluate the diffusion of the new behavioural rule. Lastly, I look at the collective welfare level reached at the end-of-time $T$. 


\subsubsection{Synchronous updating versus asynchronous updating with $\alpha=0$ : a comparison}

As mentioned, usually in the literature it is assumed that all agents simultaneously update their choices [Zeppini, 2015]. Nonetheless, in the attempt to model an institutional choice, asynchronous updating seems more appropriate. Generally, in the case of asynchronous updating, all individuals are considered to have the same chance to change their state (i.e. $\alpha=0$ ). In this section we compare this case with the synchronous updating rule. We study whether the model exhibits the same behaviour under the two distinct updating rules. This is not a priori obvious, because it is known that under specific conditions, asynchronous updating can lead to chaotic dynamics [Brock and Hommes, 1997, Zeppini, 2015].

In case of synchronous updating, the share of the population opting for $\mathrm{B}$, at $t$ is

$$
x_{t}=\frac{1}{1+e^{\beta\left[\lambda+\rho\left(1-2 x_{t-1}\right)\right]}}=f\left(x_{t-1}\right)
$$

Brock and Durlauf [2001a] and Zeppini [2015] given this map, have shown that when $\beta \rightarrow 0$, there is a unique and stable equilibrium. When instead $\beta \rightarrow \infty$, three cases can occur. If $\lambda<-\rho, x=1$ is the unique and stable steady state. If $\lambda>\rho, x=0$ is the unique and stable steady state, whereas $-\rho<\lambda<\rho$ implies that $x=\frac{\lambda+\rho}{2 \rho}$ is unstable, while $x=0$ and $x=1$ are stable. The intuition behind these results is the following. In case the intensity of choice parameter is high $(\beta \rightarrow \infty)$ and the utility differential between the choices is greater (lower) than the returns to conformity, the entire population possibly abandons (sticks to) the status quo. Contrarily, if $-\rho<\lambda<\rho$ multiple equilibria are possible. It was found that the bifurcation occurs for values of $\beta$ approximately equal to 3.5 [Zeppini, 2015].

In case the updating is occurring asynchronously, the probability of choosing institution $\mathrm{B}$, and the share of the population opting for $\mathrm{B}$, at $t$ is

$$
x_{t}=(1-\gamma) x_{t-1}+\gamma \frac{1}{1+e^{\beta\left[\lambda+\rho\left(1-2 x_{t-1}\right)\right]}}=(1-\gamma) x_{t-1}+\gamma f\left(x_{t-1}\right)
$$

where $\gamma$ identifies the proportion of agents who are randomly given the chance to possibly adjust their strategy on the basis of the discrete choice mechanism described above, whereas $1-\gamma$ is the share of people who are not entitled to revise their strategy and thus stick to the previously adopted one. $\gamma=m / N$, and $1-\gamma=\frac{N-m}{N}$, where $m$ corresponds to the number of individuals simultaneously revising their strategy, at each time period. As mentioned above, $m=1$ meaning that only one agent at the time revises his strategy.

In equilibrium, $x_{t}=x_{t-1}=x$ thus Equation 3.7 simplifies to 


$$
\begin{aligned}
& x=(1-\gamma) x+\gamma f(x) \\
& x=f(x)
\end{aligned}
$$

This shows that the synchronous (Equation 3.6) and asynchronous map (Equation 3.7) have the same stable steady states.

Figure 3.1 helps us to visualise the results. I compare the two updating rules, for two $\beta$ values and asymmetric profitability across the two behavioural options $(\lambda=0.2)$. The $\gamma$ parameter takes different values.

Figure 3.1: Comparison between synchronous and asynchronous map with random selection for different values of $\gamma$ and $\beta(\rho=1)$ and unequally profitable behaviours.
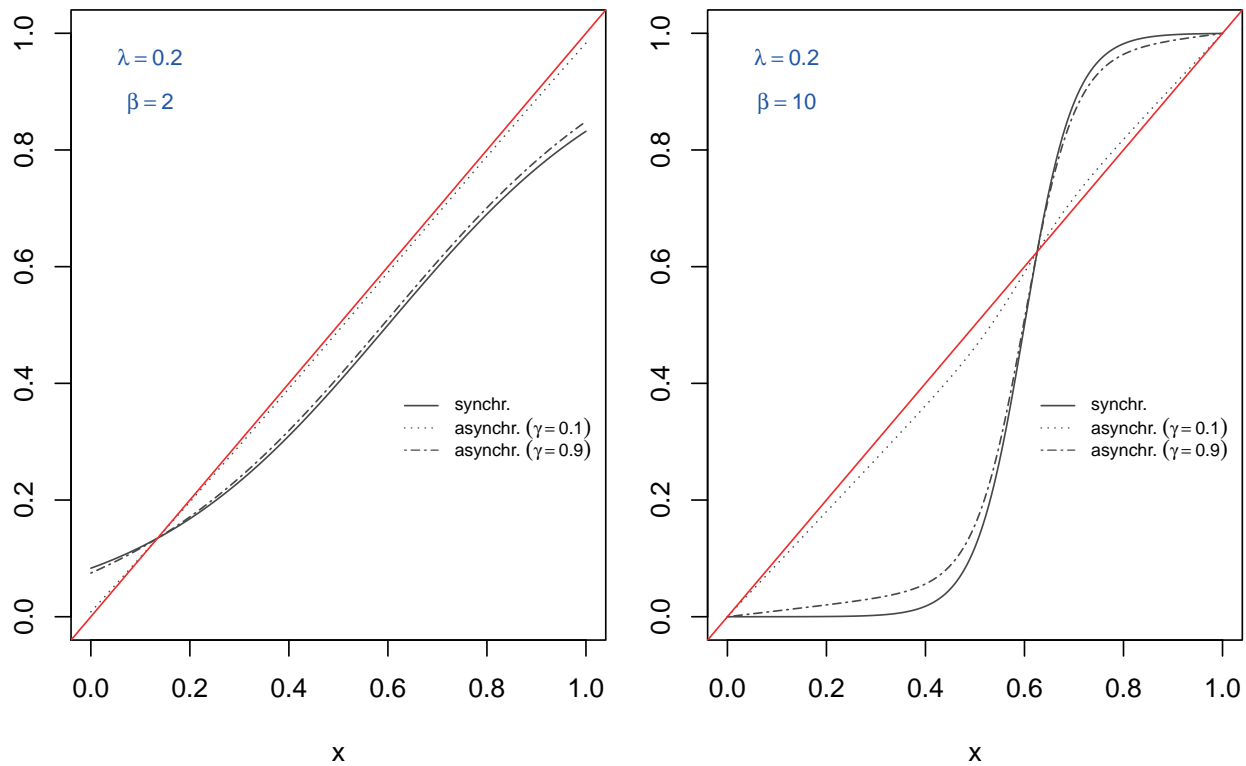

This figure shows that the $\gamma$ parameter changes the map but does not alter the fixed points and thus the steady states.

On the left hand side, for $\beta=2$, it can be seen that the old rule of behaviour is never completely replaced by the new one because some individuals still make mistakes. This means that unless individual agents see clearly the benefits associated with the new behaviour, the old one will not disappear. This holds independently from the updating rule used. For $\beta=10$, i.e. in case agents respond to the benefits being associated with the two options, $x \rightarrow 0$ and $x \rightarrow 1$ are stable states for both synchronous and asynchronous updating when $\alpha=0$. The system tends to settle on two steady state wherein one of the two options is prevalent. 
So summarising, the synchronous and asynchronous updating rules with $\alpha=0$ lead to the same set of steady states. Nonetheless, when $\alpha \neq 0$ and thus agents are selectively given the opportunity to revise their choices, this result might not hold. In the following section, I derive the new map for this case. Subsequently I analyse the long-run dynamics.

\subsubsection{Self-efficacy and asynchronous updating: the new rate equation}

As mentioned above if $\alpha=0$, agents are equally likely to revise their choice. In this section, I rather focus on the case in which one agent at a time can adjust and who this agent is determined probabilistically by his endogenously varying self-efficacy level (i.e. when $\alpha>0$ ). The goal is here to understand whether, in the long-run, the system settles on the innovative behaviour or rather sticks to the old one. For this purpose, I will first derive the new map for the case in which people are given the chance to possibly adjust their strategy depending on their self-efficacy. Then, I evaluate $x_{T}$, i.e. how many individuals within the population, at the end-of-time $T$, have not adopted the innovative behaviour. I will present a comparison between the numerical results and the theoretical predictions in section 3.4.3.

Every period $t$ one agent is given the opportunity to revise. The agent could be in one of four possible situations.

I define the probability that the selected agent $i$ is state in $\mathrm{B}$ as $w\left(P\left(X_{i, t-1}=B\right) \equiv\right.$ $\left.w\left(x_{t-1}\right)\right)$. When choosing his new strategy, the selected agent sticks to choice to $\mathrm{B}$ with probability $f\left(x_{t-1}\right),\left(P\left(X_{i, t}=B\right) \equiv f\left(x_{t-1}\right)\right)$ as defined in Equation 3.6. Otherwise he switches to option A with probability $1-f\left(x_{t-1}\right),\left(P\left(X_{i, t}=A\right) \equiv 1-f\left(x_{t-1}\right)\right)$. Alternatively, the chosen agent is in state $\mathrm{A}$ with probability equal to $1-w\left(x_{t-1}\right)$, (i.e. $\left.P\left(X_{i, t-1}=A\right) \equiv 1-w\left(x_{t-1}\right)\right)$. The probability that $i$ 's choice continues being $\mathrm{A}$ in the subsequent period is $1-f\left(x_{t-1}\right),\left(P\left(X_{i, t}=A\right) \equiv 1-f\left(x_{t-1}\right)\right)$. Conversely, the probability he switches to $\mathrm{B}$ is $f\left(x_{t-1}\right),\left(P\left(X_{i, t}=B\right) \equiv f\left(x_{t-1}\right)\right){ }^{8}$

Putting together the transition probabilities and the individual states' probabilities, it is possible to identify the following population level rate equation

$$
\begin{aligned}
N x_{t} & =N x_{t-1} \cdot w\left(x_{t-1}\right) \cdot f\left(x_{t-1}\right) \\
& +\left(N x_{t-1}-1\right) \cdot w\left(x_{t-1}\right) \cdot\left(1-f\left(x_{t-1}\right)\right) \\
& +\left(N x_{t-1}+1\right) \cdot\left(1-w\left(x_{t-1}\right)\right) \cdot f\left(x_{t-1}\right) \\
& +N x_{t-1} \cdot\left(1-w\left(x_{t-1}\right)\right) \cdot\left(1-f\left(x_{t-1}\right)\right)
\end{aligned}
$$

\footnotetext{
${ }^{8}$ For a clearer visual representation of the choice-tree please refer to the Appendix of the chapter.
} 
Equation 3.9 simplifies to

$$
x_{t}=\frac{\left(f\left(x_{t-1}\right)-w\left(x_{t-1}\right)\right)}{N}+x_{t-1}=g\left(x_{t-1}\right)
$$

To clarify how I derived this rate equation, it is worth explaining in detail all four cases. As the mechanism relies on agents self-efficacy, $w$ can be written as the ratio of self-efficacy of the individual in state B over the total self-efficacy, i.e.

$$
w=\frac{\sum s_{i, t} \mathbb{1}}{\sum_{i=1}^{N} s_{i, t}}
$$

where $\mathbb{1}$ is a dummy variable that takes value 1 if $x_{i, t}=B$.

If we define $N(B)$ and $N(A)$ as the total of the population members in $\mathrm{B}$ and $\mathrm{A}$ respectively and $\mu(B)$ and $\mu(A)$ as the average self-efficacy of these two groups $\left(\mu_{B}=\right.$ $\left.\frac{\sum s_{i} \mathbb{1}}{N(B)}\right)$, we can rewrite $w$ as

$$
w=\frac{N(B) \mu(B)}{N(B) \mu(B)+N(A) \mu(A)}=\frac{1}{1+\frac{N(A)}{N(B)} \frac{\mu(A)}{\mu(B)}}
$$

As $x_{t}=\frac{N(B)}{N(B)+N(A)}$, we can write

$$
w\left(x_{t-1}\right)=\frac{1}{1+\frac{1-x_{t-1}}{x_{t-1}} \frac{\mu(A)}{\mu(B)}}
$$

There is one factor in Equation 3.13 which remains to be explained, namely the ratio between $\frac{\mu(A)}{\mu(B)}$. In order to gain further insights on how $\mu(A)$ and $\mu(B)$ can be related, one can consider the specific case in which agent $i$ changes his state. Suppose that agent $i$ switches from B to A. Then, according to Equation 3.5 and omitting the normalisation factor, the new self-efficacy level $i$ obtains, given that he is now in state $\mathrm{A}$, is equal to the product between the self-efficacy he obtained from being in state $\mathrm{B}$ times $e^{\alpha(\Delta u)}$ that is $s_{i, t}(A)=s_{i, t-1}(B) * e^{\alpha\left[\lambda+\rho\left(1-2 x_{t-1}\right)\right]}$. We can say that

$$
\mu(A)_{t}=\mu(B)_{t-1} e^{\alpha\left[\lambda+\rho\left(1-2 x_{t-1}\right)\right]}
$$

Using Equation 3.13 and 3.14, we can write

$$
w(x)=\frac{1}{1+\frac{1-x}{x} \cdot e^{\alpha[\lambda+\rho(1-2 x)]}}
$$


Here we assume a stationary state and we will check with simulations whether this is true. In section 3.4.3 I will provide numerical evidence that this holds and $w(x)$ is time invariant and that Equation 3.15 well predicts the results.

Agent $i$, once chosen, can decide to stick to his previous choice, or switch to the alternative one. This choice is independent from the state in which he was before. The probability that he chooses option B is $f\left(x_{t-1}\right)$ as defined in equation 3.6. The probability that $i$ 's choice is instead A at $t$ is $\left(1-f\left(x_{t-1}\right)\right)$. Now that we have analysed in detail the four cases that characterise the process and have derived the new rate equation, we can, in section 3.4.3, derive the theoretical steady states and compare them with the simulation results.

\subsubsection{Steady States}

Assuming it exists, in the steady state $x_{t}=x_{t-1}=x$, thus we can re-write Equation 3.10 as follows

$$
\begin{aligned}
N x & =f(x)-w(x)+N x \\
f(x) & =w(x)
\end{aligned}
$$

Substituting into Equation 3.16, Equations 3.15 and 3.6 and re-arranging to gain more insights we find that

$$
x=\frac{1}{1+e^{(\beta-\alpha)[\lambda+\rho(1-2 x)]}}
$$

Equation 3.17 shows that in equilibrium, $x$ depends on all the parameters and more interestingly it is the difference between $\beta$ and $\alpha$ that differentiate my rate equation from Zeppini's [2015] and Brock and Durlauf [2001a].

As a next step, I will use the rate equation to derive the theoretical predictions. In Figure 3.2, I present the long-term evolution of the share of individuals choosing the less rewarding option when the returns to conformity are high $(\rho=1)$. The upper panels of Figure 3.2 show the time series for $\alpha=0$. Differently, the lower panels report the case in which the reinforcement in self-efficacy is high $(\alpha=3)$. In both cases I consider two values of $\beta$. The red dashed lines identify the theoretical steady states. 


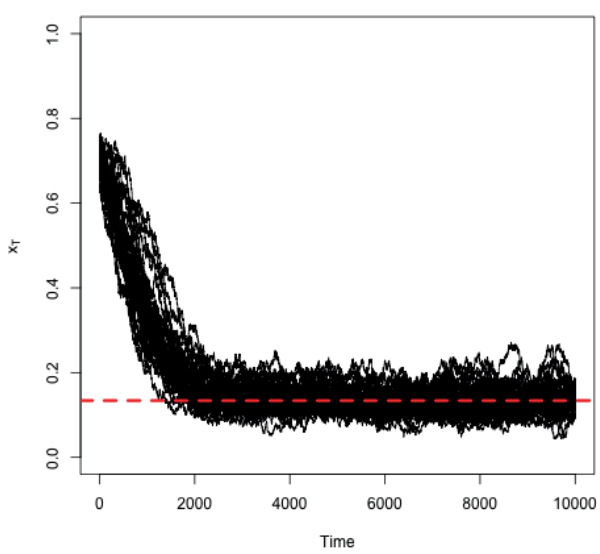

(a) $\beta=2, \alpha=0$

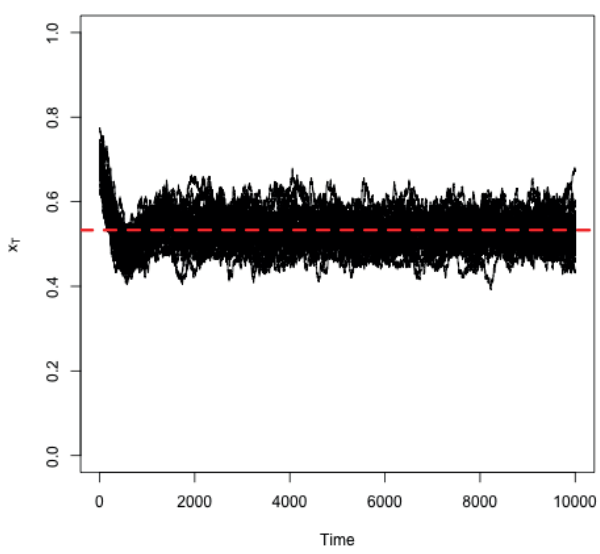

(c) $\beta=2, \alpha=3$

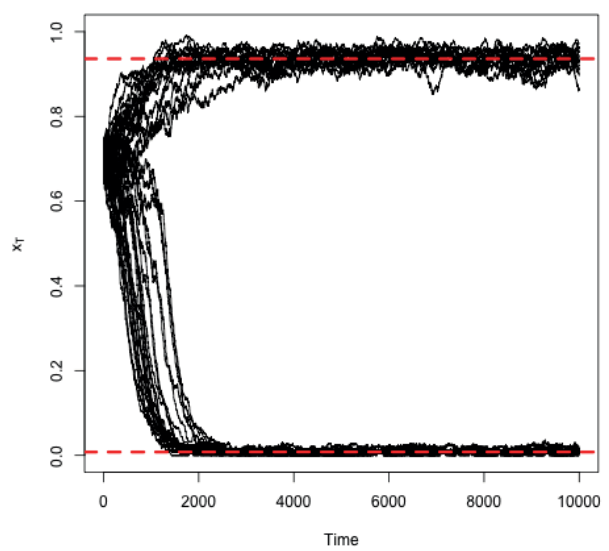

(b) $\beta=4, \alpha=0$

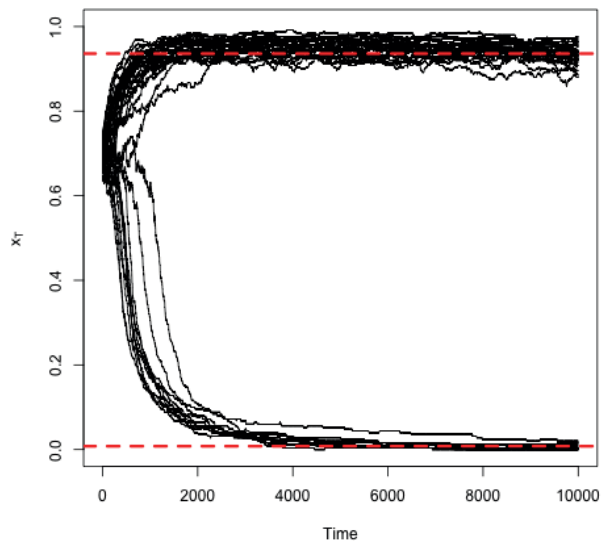

(d) $\beta=7, \alpha=3$

Figure 3.2: Time series of the proportion people sticking to the old behavioural rule $\left(x_{t}\right)$ for 2 different values of $\beta$ and $\alpha, \rho$ is 1.40 iterations.

These time series show that for $\beta \rightarrow 0$ both options coexist regardless of the way in which people are given the chance to update their state. This confirms and expands on Zeppini's [2015] result. For higher values of $\beta$, independently from whether people are equally likely to revise their choice $(\alpha=0)$ or only few take the chance to do so $(\alpha \neq 0)$, a bifurcation occurs. Some of the simulations converge to a low proportion of less rewarding options, some others instead result in a high share of individuals opting for 
the old behavioural rule. ${ }^{9}$ Whether some simulations converge towards $x_{T}=1$, or $x_{T}=0$ depends on the initial conditions. ${ }^{10}$

Figure 3.3 instead shows the outcome of the comparison between the theory and simulations. It reports on the y-axis the end-of-time proportion of individuals sticking to the old option whereas on the $\mathrm{x}$-axis different $\beta$ values are reported. In each panel, I use different levels of returns to conformity $(\rho)$ and I compare the results under different self-reinforcement regimes, i.e. $\alpha$ values, as reported in the legend. $\lambda$ is equal to 0.2 .

Figure 3.3: End-of-time proportion of individuals choosing the old behavioural rule (averages across 200 iterations) for different values of $\beta$ and different self-efficacy self-reinforcement regimes $(\alpha)$
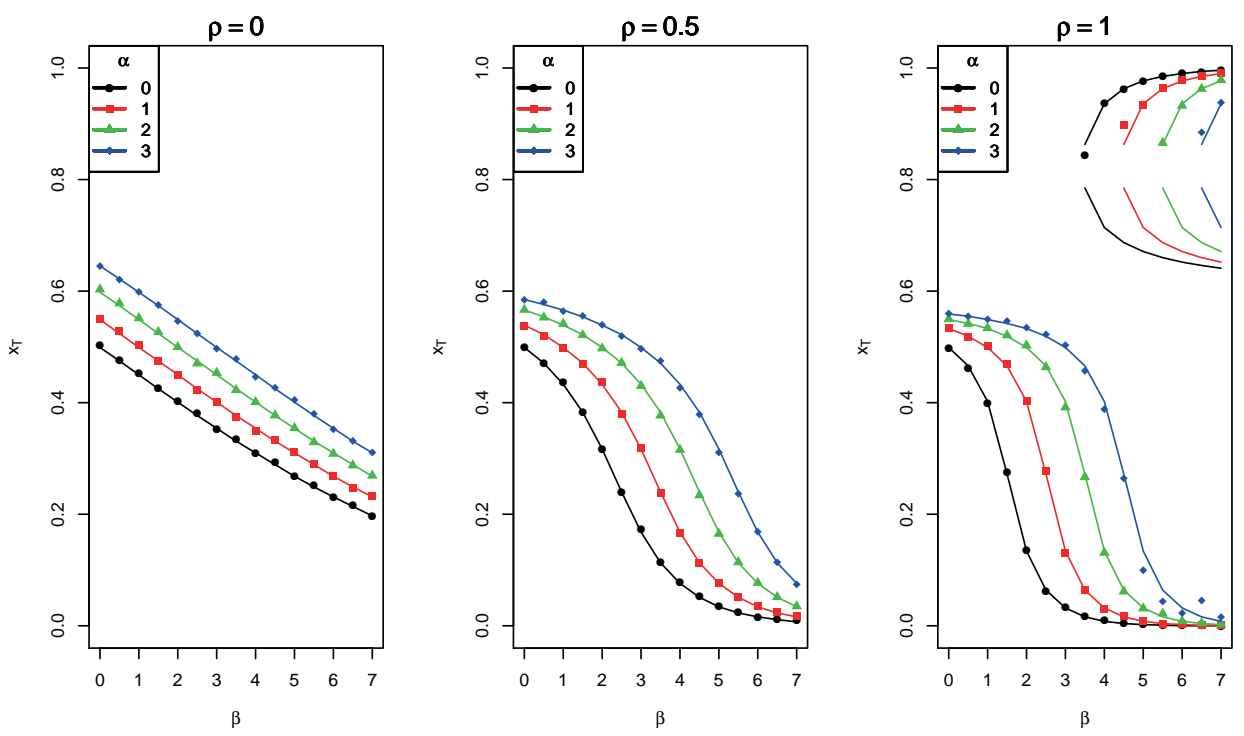

In each panel, the solid lines represent the theoretical equilibrium predictions that one derives using the rate equation (Equation 3.10). The dots identify the simulation results.

\footnotetext{
${ }^{9}$ Given the presence of these bifurcations, it is worth briefly discussing how I calculated the average $x_{T}$ across simulations in case $\rho=1$ in order to proceed with the graphical representation in the right panel of Figure 3.3. As some of the simulations go up towards $x_{T}=1$ whereas some others go down towards $x_{T}=0$, I use the repulsive equilibrium to discriminate amongst all numerical realisations. I average all the end-of-time share of the population adopting the new rule of behaviour depending on whether they fall below or above the repulsive equilibrium.

${ }^{10}$ Initial conditions are always drawn at random from a uniform distribution. In this Figure, purely for visualisation purposes and to show the existence of the bifurcation, I bias the initial conditions towards a majority of individuals starting off in state A.
} 
These results were obtained averaging 200 iterations for 250 agents ${ }^{11}$, 25000 periods, initial homogeneous self-efficacy of 0.5 , and several values of $\beta, \rho$ and $\alpha .{ }^{12}$

Looking at Figure 3.3 it is evident that the simulation results seem to perfectly match the theoretical predictions. Moreover, in case of low returns to conformity $(\rho=0$ or $\rho=0.5)$, the share of wrong choices declines with the increase in the intensity of choice parameter. This is trivial. It implies that as the probability of making mistakes declines (i.e. $\beta$ increases), agents are more likely to switch to the new state. Thus, the innovative behaviour becomes the most diffused.

But there are two main results that Figure 3.3 is able to show.

First, as expected in case of high returns to conformity $(\rho=1)$, as it can be seen on the right panel, for $\beta$ values above 3.5, independently from the value of $\alpha$, a bifurcation occurs. The system can equivalently end up in either of two possible equilibria: we can observe a high diffusion of the innovative behaviour or high resistance to change. This means that in case returns to conformity are high, an increase in the intensity of choice parameter (higher $\beta$ ), is not sufficient to guarantee a complete diffusion of the new social innovation. Moreover, it can be noticed that, when agents revise their choices depending on their self-efficacy the bifurcation is shifted to the right. This means that for $\alpha>0$, the bifurcation occurs for values of $\beta$ equal to approximately $3.5+\alpha$. The exact level of the intensity of choice depends on the intensity of the self-reinforcement in self-efficacy: the higher the self-reinforcement, the lower the probability of mistakes. As shown in the right panel of Figure 3.3, when $\alpha=0$ the bifurcation occurs for $\beta=3.5$, whereas for $\alpha=3$, the bifurcation takes place at $\beta=6.5$. Thus, the exact value of $\beta$ for which the bifurcation occurs is different depending on the intensity of the self-reinforcement in self-efficacy (i.e. $\alpha)$.

Second, for low pressure for conformity, when only the most self-efficacious individuals can actively make the choice, a higher proportion of individuals ends up choosing the less rewarding behaviour. This is a somehow counterintuitive and very interesting result which can be explained as follows. When $\alpha>0$, agents with higher self-efficacy have higher chances to revise their choice and thus switch from the old to the innovative behaviour. If the switching occurs, it means that agents gain in welfare and self-efficacy and the probability of enacting the change in the future increases again. All other individuals instead stick to their previously adopted strategy, which might have been wrong. ${ }^{13}$ For

\footnotetext{
${ }^{11}$ I want to clearly state that $N$ must be large. This is especially true for $\alpha>\beta$ because otherwise in this case the map is non-monotonic.

${ }^{12}$ I limit my exploration to certain values of the parameter space. Specifically, I limit the value of the self-reinforcement parameter to 3 and $\beta$ equal to 7 . I nonetheless explored also instances in which $\alpha \geq 4$ and in which both $\alpha$ and $\beta$ are large. My results seem to hold in these cases, nonetheless convergence time visibly increases.

${ }^{13}$ Each individual is randomly assigned a state at time zero. Given the mechanics of the model, some individuals might be given the wrong state and possibly stick to it because they are hardly ever
} 
this reason, we observe that even when the probability of making mistakes is low (high $\beta$ ), the share of wrong choices is higher than in case $\alpha=0$ and all population members have equal chances to enact change and revise their strategy. Self-efficacy increases people's ability to engage in action by allowing them to choose between certain behavioural rules. This creates heterogeneity in the opportunities people seize. While some individuals might be constantly willing to revise their strategy, a portion of the population, possibly large, does not change its choice nor improve its self-efficacy. This is because an increase in agent's $i$ self-efficacy, reduces the probability that other people revise their choices. This is the key mechanism driving my result: individual willingness to engage in institutional change does not only depend on personal past success, but also the past successes/failures of others.

These statements are supported by the results shown in Figure 3.4. In panel (A) I plot, for different time periods, identified by the thickness of the lines, the density function of self-efficacy for the two groups of individuals: those choosing the innovative behaviour shown by the dashed green lines, and those sticking to the old rule in solid red. I use a kernel density estimator to draw this plot. In panel (B) I plot the theoretical values of $w\left(x_{t}\right)$ (black solid line) against the numerical realisations (red line). Panel (C) shows the evolution of the mean self-efficacy of the group of individuals choosing option A (green line) and B, the least rewarding option.

update their strategy. By the same logic, some individuals might, by chance, be assigned the correct behaviour and subsequently have not the chance to revise. As a consequence, "luck" at birth, or the lack thereof, is in this model path-dependent. This feature is not unrealistic and it recalls one of the building blocks of a recent model elaborated by Ahuja et al. [2017]. In their model, individuals are born as high or low quality workers. At death, they leave an inheritance to their offsprings. And high quality individuals are more likely to leave a larger bequest than low quality individuals. 
Figure 3.4: Density function of self-efficacy for those individuals choosing the innovative behaviour shown by the dashed green lines, and those sticking to the old rule in solid red (panel a). Evolution of theoretical (black line) and numerical (red line) $w$. Horizontal blue dashed line represents $w$ as a function of the equilibrium value of $x$ (panel b). Time series of mean self-efficacy of the individuals choosing option A (i.e. $\mu(A)$ ), here represented by the green line, and $\mu(B)$ dashed red line (panel c). One iteration, $\beta=2, \rho=1, \alpha=3$ and $N=250$.
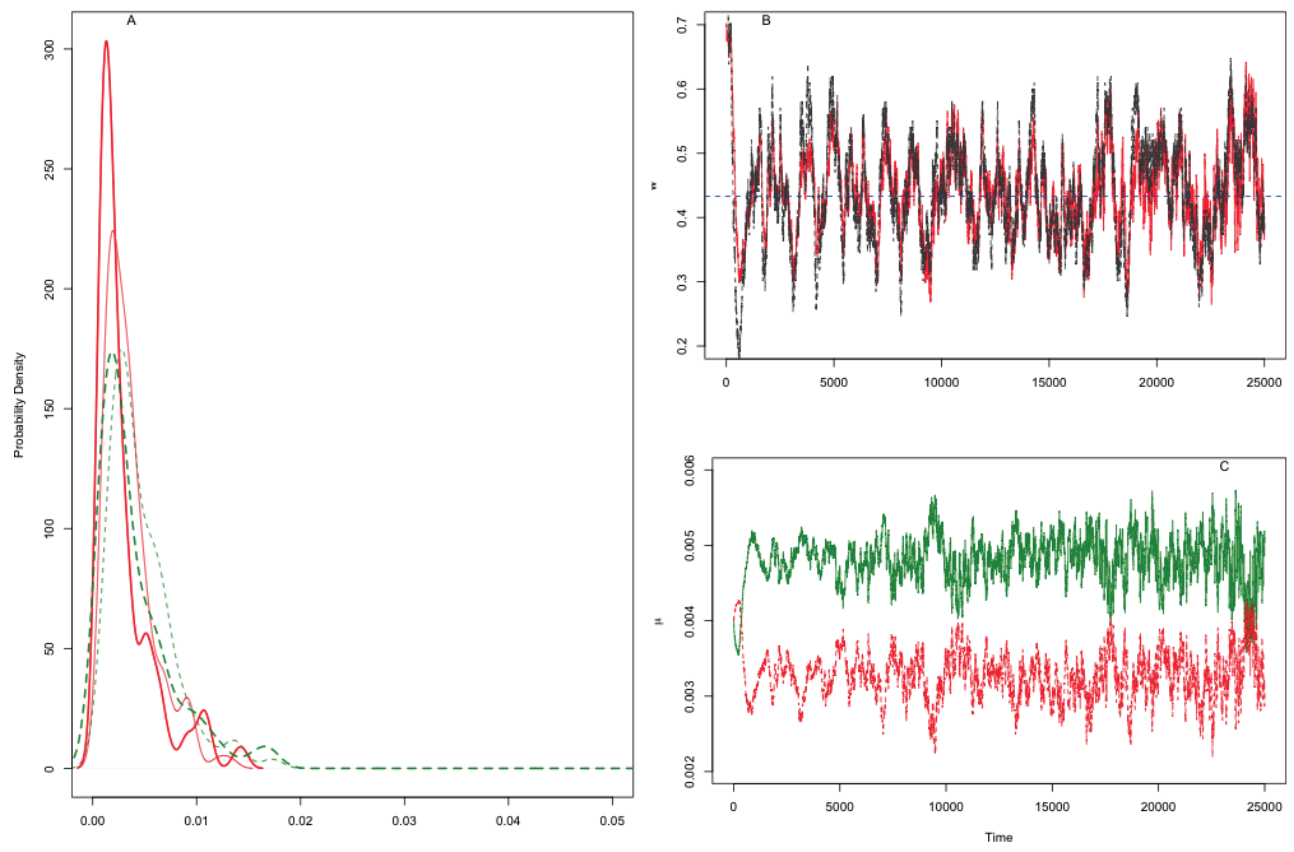

It can be seen in panel (A) that those individuals choosing the more rewarding option are more likely to have higher self-efficacy than those choosing the old behavioural rule. Over time the probability mass shifts. Panel (B) shows the time series for $w$. The red line plots the numerical realisations of Equation 3.11, the black line instead plots Equation 3.15 evaluated at $t$. The horizontal blue dashed line plots the value of $w$ for the equilibrium value of $x$ (i.e. $w\left(x^{*}\right)$ ). In panel (C) I plot the time evolution the mean self-efficacy of those individuals choosing the wrong option (red line) and of those instead opting for the more rewarding one (green time series). Both groups experience a constant average selfefficacy, but as expected the group choosing option A maintains over time higher average self-efficacy levels. The close dynamics of the numerical and theoretical values of $w$ shown in panel (B), together with the constant ratio between $\mu(A)$ and $\mu(B)$ shown in $(\mathrm{C})$, offer support for the validity of Equation 3.15 and, consequently, for Equation 3.14. 


\subsubsection{Collective welfare}

In order to corroborate the results shown in Figure 3.3, I also analyse collective welfare. Figure 3.5 shows the end-of-time collective welfare level for different $\beta$ values and levels of network externalities. I plot the results for the highest self-reinforcement in self-efficacy $(\alpha=3)$ and for case in which self-efficacy does not change and all members of the population have the same chances to revise their state $(\alpha=0) \cdot{ }^{14}$ The solid lines identify the theoretical predictions, the dots are the simulation results.

Figure 3.5: End-of-time collective welfare (averages across 200 simulations) for different values of $\beta$ and two different self-efficacy self-reinforcement $(\alpha) . \lambda$ is set equal to 0.2 .

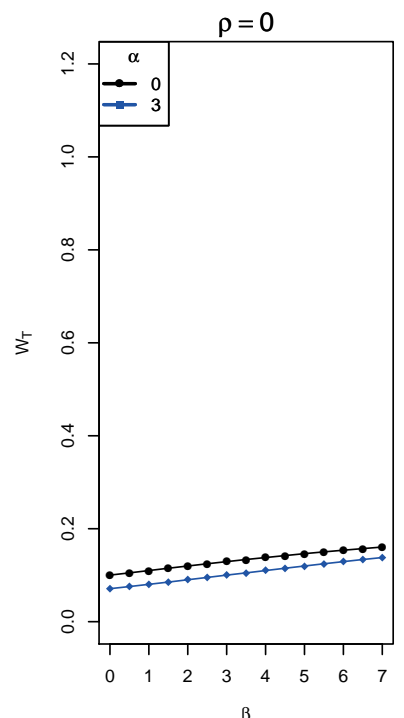

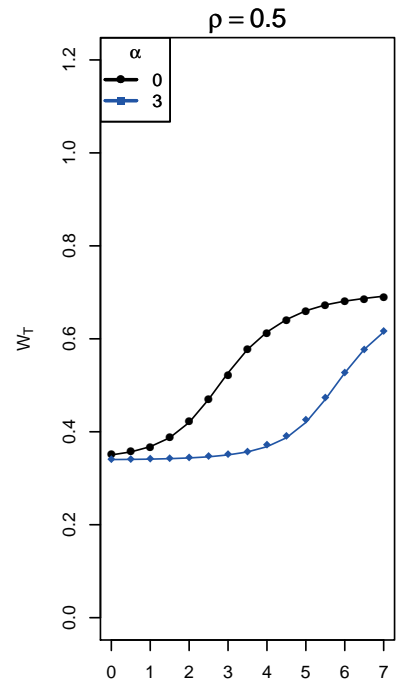

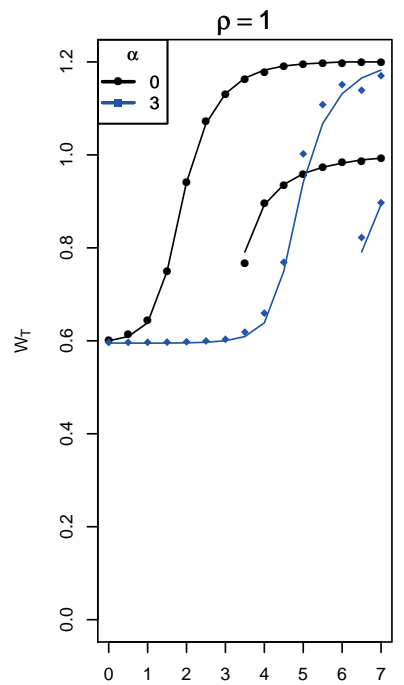

As expected from the results shown in Figure 3.3, welfare increases with an increase of in the intensity of choice, in case of low or null returns to conformity ( $\rho=0$ and $\rho=0.5)$. Under these circumstances, high variance in self-efficacy is surely detrimental for welfare. Notably, higher self-reinforcement in self-efficacy leads to lower collective welfare levels. Again, given the mechanics of the model, most individuals "freeze" and rarely modify their possibly inefficient choice. Therefore, the system settles on lower collective welfare levels.

${ }^{14}$ The cases of $\alpha=1$ and $\alpha=2$ are omitted for readability. The corresponding welfare levels reached at the end-of-time lie right in between the curves displayed in here. 
The most interesting finding is displayed in the right panel of Figure 3.5 wherein returns to conformity are high $(\rho=1)$. From above, we know that for certain values of $\beta$ a bifurcation occurs. Thus, following the procedure used above, in these circumstances I calculate welfare using the repulsive theoretical equilibrium and averaging all those simulations that at the end-of-time stand above or below this point.

We can observe that when everybody has the same opportunity to enact change collective welfare is higher, but not if $\beta$ is greater than 5 . In this region of the parameter space, due to the bifurcation, some of the simulations might converge towards the highest absorbing steady state, some others instead might descend towards the lowest one. In this case, when $\alpha=3$ the average welfare reached by the simulations converging towards $x_{T}=1$, is higher than the welfare obtained with $\alpha=0$ if the simulations tend towards $x_{T}=0$. In fact, the blue line lies above the lowest of the two black lines.

\subsection{Conclusions and possible extensions}

This paper offers a formal account of the (non-)evolution of institutions. Specifically, using psychological insights on the evolution of individual beliefs concerning personal abilities to enact change, I describe how the "rules of the game" might (fail to) change. I rely on a well-established social interaction framework and compare the case in which all population members have equal chances to enact the change to the case in which only few individuals have this opportunity.

By means of simulations I show that, when not all agents have the same confidence in being able to revise their choices, institutional resistance is higher. When only few individuals take the opportunity to change their state, whereas most population members stick to the status quo, a complete behavioural shift is unlikely to occur. Low self-efficacious individuals can prevent trendsetters to achieve the desirable institutional change. This implies that the social innovation diffuses to a lesser extent and collective welfare is lower. Additionally, in line with previous literature I confirm that high pressure for conformity and lower probability of mistakes triggers multiple equilibria: societies possibly end up either adopting the new institution or get trapped into the status quo. The extent to which the social innovation diffuses depends on the difference between the probability of making mistakes and the reinforcement in self-efficacy.

It is worth acknowledging that the rule used to update individuals' confidence levels might not apply to all circumstances but still represents a good option. Aside from the evident modelling advantages, there exists robust evidence of the fact that people often judge their abilities to accomplish something and thus their confidence relative to others. 
Self-efficacious individuals are more likely to self-select to enact change. This is why such dynamics has been chosen.

I have elaborated the simplest possible version of this model, but it could surely be further extended in multiple ways. My model totally disregarded spillovers. Self-efficacy generally stems from the continuous integration of information from different sources. One of these sources is social learning. Observing close individuals failing or succeeding affects people's judgements on one's capabilities to achieve certain goals. Friends' performance represents a benchmark to judge the likelihood of one's success or failure, i.e. to assess personal capabilities of attaining the same goal. By the same token, watching someone failing undermines one's self-efficacy. Thus, taking this into account would provide a more exhaustive picture of how human agency affects institutional innovation processes. I believe that an extension along these lines would be very valuable and would naturally pave the way towards the integration of specific network structures into the model. In fact, my model considers a full network topology. This is the simplest structure to carry this exercise, moreover some exploratory analysis conducted with a scale-free and small world topology seems to suggest that there is not qualitative change in the results. Nonetheless, when considering self-efficacy spillovers the network structure becomes more important and a more rigorous exploration of multiple topologies would be required.

The second important change to be possibly made to this model is to endogenise also the intensity of choice parameter of the logit model. Here, I considered it to be the same for every member of the population and to be static. This is a rough approximation of reality. Each individual might make mistakes with different probabilities and might learn at a different rate. Moreover, in this paper I remained agnostic concerning the interpretation given to $\beta$, but one possibility, given this framework, is to interpret it as individual judgments of the likely consequences the changing from one behaviour to another will produce [Bandura, 1997]. Low values of $\beta$ would indicate that people believe that their action does not carry any weight onto reality, high values of $\beta$ instead would be synonym of high confidence in the possibility of making a real difference. This is what Bandura [1997] defines as outcome expectancies.

Another possible modification to this model relates to the number of agents entitled to revise their strategy in every period. As mentioned above, there are theoretical reasons for choosing the asynchronous updating rule to describe institutional dynamics. Nonetheless, one could imagine a model in which, instead of only one single individual revising his strategy, an endogenous number of agents do so each period. Each agent would decide probabilistically whether to make the effort to reconsider, and the probability of reconsidering depends on the agents self-efficacy. In this case there would be a feedback both at the individual level, from experience to self-efficacy and thus to the probability of re- 
considering strategy, but also at the aggregate level, in which agents' experiences, affecting their self-efficacy and thus the probability of re-considering, have a further, system-level, impact on how many agents are active each period. It is known in the literature that, given a non-monotonic map, asynchronous updating might lead to chaotic dynamics (see Figure 7 in Zeppini [2015], Hommes [1994] and Diks and Van Der Weide [2005]). Whether or not chaotic dynamics do emerge depends in part on the proportion of agents who revise strategies each period. In the example reported in Zeppini [2015], when the proportion of people revising their strategy lies between 0.5 and 0.75 , chaos arises. In the variant described just above, this proportion can change from period to period, and is indeed endogenous. A priori it is unclear what the dynamics of such a system would look like, but the introduction of a second effect of the dynamic self-efficacy makes the system more complex, possibly leading to richer dynamics, and would thus be an interesting domain to explore.

Despite the simplicity of the model, the results I obtained are very interesting and relevant. In general, the model shows that the presence of low self-efficacious individuals can represent an obstacle for institutional change. In communities wherein people give little weight to others' actions, and only few pro-active individuals try to enact change, a complete behavioural shift is unlikely to occur. The majority of the population sticks to the standing behavioural rule. This hinders social innovation as few trendsetters are unable to achieve the desired change. As a consequence, collective welfare stagnates. When the pressure for conformity is low, a plausible way to increase societal welfare consists reducing the probability of making mistakes. Alternatively, a widespread and equitable increase in individuals' perceived capabilities to enact change would be helpful to reach higher collective welfare levels. If people give little weight to the choices made by others, increasing people's aspirations and confidence in their capacities to seize the opportunity to defy the status quo would lead to welfare gains.

My analysis also confirms that when the pressure for conformity is high, a lower probability of mistakes, is not a sufficient condition to guarantee the diffusion of the social innovation. In these circumstances society can either get trapped into the status quo or fully adopt the newly available behaviour. Differently from what was already known in the literature, my model shows that the occurrence of these events depends on the intensity of the self-reinforcement in self-efficacy. This concretely implies that when, within a close-knit community, people's self-efficacy deteriorates at a fast pace as a result of past failures and only very few trendsetters withstand the current behavioural rule, multiple equilibria arise even if the likelihood of making mistakes is very low. This is interesting because it shows that, in close-knit societies, trendsetters, despite possibly making few mistakes, are unable to prevent institutional lock-in. 
3 How do institutions change? Self-efficacy Beliefs and Social Interactions

\subsection{Appendix 1: The decision tree}

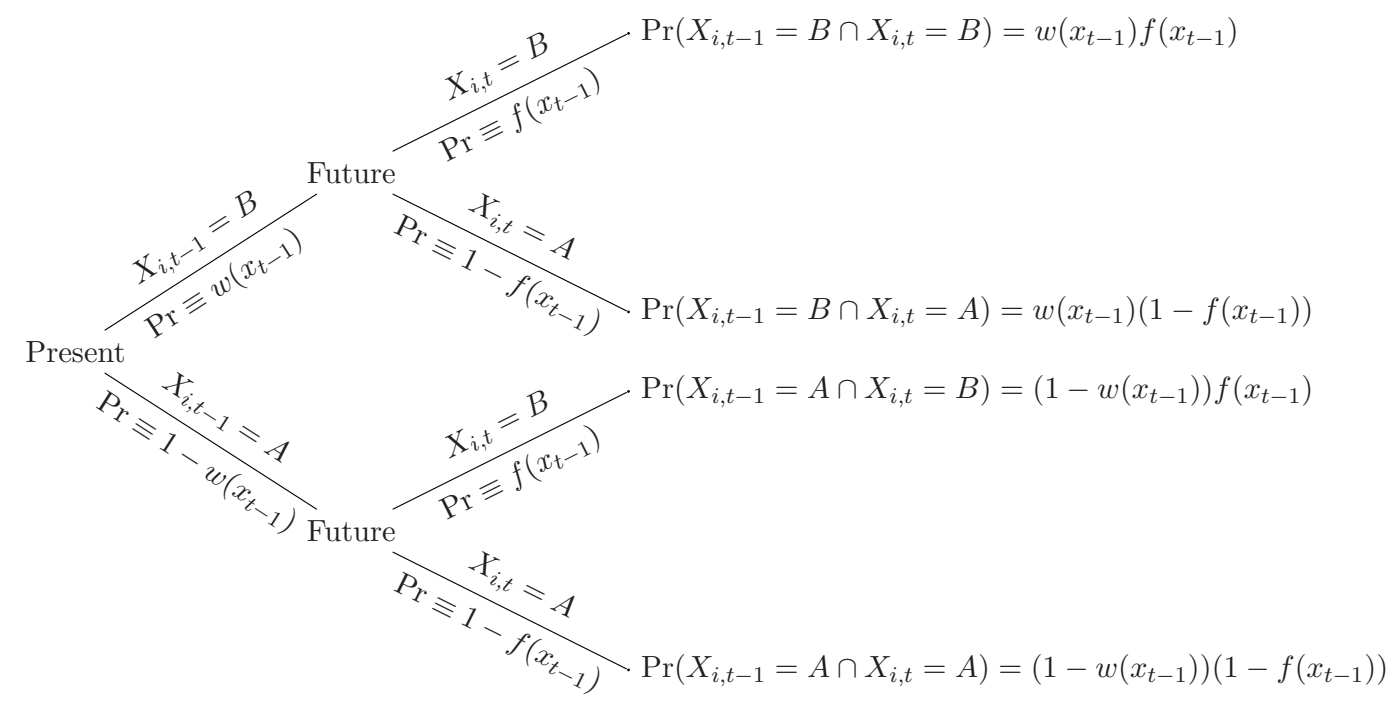




\title{
CHAPTER 4
}

\section{Mental models and Institutions ${ }^{1}$}

\begin{abstract}
The importance of institutions on the economic trajectories countries might undertake has been widely acknowledged. This chapter explores the nexus between mental models and institutions. Exploiting countries' differences in perceived behavioural control as a proxy for mental models degree of malleability, I show that mental models and institutions co-evolve. I find that a ten percentage points increase in a country's freedom of choice will improve its rule of law by 0.32 , allowing the country to move from the rule of Tunisia to the rule of law of Italy in 2015. When controlling for country specific characteristics, this effect reduces by one order of magnitude. In the second part of this study, I investigate whether mental models and institution have an independent effect on economic development. Using a pooling model, I find that an increase in ten percentage points in perceived freedom, generates a 5 per cent increase in per capita income, when controlling for institutional quality. Instrumental variable regressions provide weak evidence for this effect.
\end{abstract}

${ }^{1}$ I would like to thank Nicolò Bellanca, Robin Cowan, François Lafond and Eleonora Nillesen for their comments and suggestions. The author also thanks Nuffield College for providing access to the Gallup Analytics dataset. 


\subsection{Introduction}

Differences in average income levels across nations are still at the centre of an animated political and academic debate. Geography, trade integration and institutions are usually considered as the determinants of economic growth and arbitrate which society will innovate, accumulate and thus develop and which will not. A seminal paper by Rodrik et al. [2004] elaborated a layered explanation of economic development and aimed at discovering which one of these three elements matters the most. It was found that institutions trump trade and geography in determining countries' development paths. More recently, Acemoglu et al. [2014] argue that democratic institutions have a robust and positive effect on income. It is beyond the scope of this study to re-establish the importance of these findings. In this chapter, I rather explore the role that agents' mental models ${ }^{2}$ play in stimulating institutional innovation and possibly explain long-term growth.

The questions this study wishes to answer are the following: Can mental models, through their effects individual introspective beliefs of behavioural control, condition institutional quality? And do mental models affect economic development?

In the first part of the chapter, I focus on the mental models and institutions nexus. The idea that mental models is a key ingredient in the institutional evolution can be traced back to Denzau and North [1994] and Mantzavinos et al. [2004]. According to North [1994, p.363] "the relation between mental models and institutions is an intimate one". While mental models are the internal representations that individual cognitive systems elaborate to portray the world's functioning, institutions are the external mechanisms individuals build to order and systematise the environment. Therefore, according to Denzau and North [1994] institutional change reflects the evolution of mental models. But, if mental models are rigid, strongly prescriptive and hard-to-eradicate then institutional innovation might be less likely to occur. Yet, few scholars have empirically investigated the effect of mental models' stickiness on the institutional evolution. In fact, it is difficult to measure mental models' malleability.

Drawing on the psychology literature, I proxy mental models' stickiness with individual perceived behavioural control levels. In fact, mental models determine which behaviour is socially plausible and acceptable in a given situation. Specifically, they condition how much freedom of choice individuals have and how much control they feel over the outcome of their actions given the environment wherein they act. Psychologists have defined

\footnotetext{
${ }^{2}$ When using the term mental models I follow the definition provided by Denzau and North [1994]. Mental models represent the concepts and categories that individual use to make sense of the world. These are not individually elaborated but rather reflect the shared understandings of the community individuals belong to. As they are shared by everyone around, individual tend not to question these views. Similar concepts which are usually referred to as schemas or cognitive frames [DiMaggio, 1997, Markus, 1977] have been used also in psychology, sociology, anthropology and political science.
} 
these beliefs as perceived behavioural control. Perceived behavioural control refers to whether individuals consider the performance of a behaviour to be possible and under their voluntary control [Ajzen, 2002]. Low perceived behavioural control triggers conformism and discourages dissent. Conversely, high perceived behavioural control corresponds to personal freedom and promotes personal accomplishments.

As a consequence, one can expect that in countries wherein, on average, people experience a low sense of control over the possibility to defy the mental models' prescription, the institutional evolution occurs at a slower pace.

I bring this argument to the data and test whether mental model malleability affects institutional quality. I use two different measures of perceived behavioural control as a proxy for mental models' stickiness and use panel data techniques to estimate their effects on institutional quality. I find that a ten percentage points increase in a country's "freedom of choice" will improve its rule of law by 0.32 , allowing the country to move from the rule of Tunisia to the rule of law of Italy (in 2015). When controlling for country specific characteristics, this effect reduces by one order of magnitude.

In the second part of the chapter, I test whether an independent effect of mental models and institutions on growth exists. Using a pooling model, I show that mental models seem to considerably affect growth. When considering country and time fixed effects a ten percentage point increase in mental models' malleability leads to a 5 percent increase in income per capita. Given the scarce variation over time and the possible endogeneity problems, I use instrumental variables regressions. A sound and foolproof instrument for mental models is hard to find. Similarly to Gorodnichenko and Roland [2017], I construct an ad hoc measure of genetic distance, based on frequencies of blood types, between the population in a given country and the population in Norway, the country wherein on average people feel the highest level of perceived behavioural control in my sample. I use the conventional settler mortality measure as an instrument for institutional quality. Given that both instruments are time-invariant, I rely on crosssectional regressions and provide some weak evidence which suggests in some cases mental models condition economic growth.

This chapter contributes to several streams of literature, namely institutional economics, development economics and the new literature on cultural economics. First, I empirically test the theoretical framework used by Denzau and North [1994] and, Mantzavinos et al. [2004]. I use psychological concepts which have recently attracted the attention of economists [Fehr and Hoff, 2011, Hoff and Pandey, 2014, Hoff and Stiglitz, 2010] as a proxy of mental models. I also contribute to the growing literature on culture economics. This literature claims that high attention has been given to institutional structures, but too few scholars have been focusing on both institutions and mental models [Alesina and 
Giuliano, 2015]. For this reason in the second part of the chapter, I test whether mental models and institutions exert an independent effect in determining the path of growth that countries undertake. The chapter proposes a richer view of the development process, it acknowledges the role of institutions and underlines the importance of individual perception of freedom.

The remainder of this chapter is organised as follows. Section 4.2, building on relevant empirical and theoretical literature from psychology and economics, synthesises the theoretical framework. Section 4.3 provides some details on the data and elaborates on the main variables of interest. Section 4.4 presents the main results: mental models and institutional quality co-evolve. Section 4.5 sheds some light on the relation between the degree of malleability of mental models and economic development. Section 4.6 concludes and underlines the importance of this empirical exercise, acknowledges all limitations and discusses possible future research along these lines.

\subsection{Related literature}

In this section, I synthesise how mental models can affect institutions and development. I formulate the argument focusing on general themes documented by previous studies in psychology and economics.

Institutions are considered an important factor in explaining economic phenomena and variations in economic growth. Although long-term development is generally considered a complex phenomenon, it is widely acknowledged that institutions represent the primary factor which affects the wealth of nations [Acemoglu et al., 2014, Acemoglu and Robinson, 2012, Rodrik et al., 2004]. Most scholars have used the growth regressions framework to establish this point. Acemoglu et al. [2001] argue that developing extractive institutions has long-lasting negative effects as elites are able to consolidate their power and thus inequality. Conversely, developing inclusive institutions is more likely to promote equality and therefore sustainable development in the long-run. Rodrik et al. [2004] elaborated a layered explanation of economic development and showed that, on a cross-sectional basis, institutions trump geography and trade integration. Easterly and Levine [2003] estimate regression models of income levels on some measures of endowments, institutions, and "policies". They find that institutions have an effect on growth, whereas endowments affect development only through their effect on institutions. More recently, Acemoglu et al. [2014], using panel data, highlight the role of democratisation processes on longterm growth. Several scholars also have investigated the role of specific cultural traits relevant for development [Fernández, 2008, 2011, Guiso et al., 2006]. Nonetheless, "those who write about institutions do not seem to worry much about whether institutions are 
well identified and isolated from cultural influences, which may be problematic" to assess the role of institutions on the wealth of nations [Alesina and Giuliano, 2015, p.899]. ${ }^{3}$ More specifically, little attention has been devoted to the role played by the co-evolution of mental models and institutions on growth.

In order to interpret reality, individuals use mental models. They represent concepts, categories, causal narratives, ideologies, or world views. They can be thought as the spectacles or windows though which we observe the world and consequently act upon it. They are the prediction that the mind elaborates, or the expectation that the mind has concerning external environments. Mental models emerge and reproduce themselves through learning [Mantzavinos et al., 2004]. Evidence suggests that mental models are passed down over generations [Alesina and Giuliano, 2015, Greif and Tabellini, 2010, Guiso et al., 2006].

In the economics literature, mental models are thought to determine how individuals construe a situation and which actions seem plausible and desirable in that situation [Hoff and Pandey, 2014]. Mental models capture the beliefs concerning the world's functioning. In a recent experimental paper, Bernard et al. [2011] report that disadvantaged individuals in Ethiopia feel that their future is pre-determined, their freedom of choice and control over the outcome of their actions is scarce. They claim they "have neither a dream nor an imagination". These limit their ability to seize available opportunities, to invest in productive assets to change their status and to achieve a better future. Another set of recent experiments confirms that mental models can affect individual sense of control, selfevaluation and thus, the ability and motivation to perform [Hoff and Pandey, 2006, 2014]. Individuals, belonging to a mixed caste groups, were asked to solve mazes. In a control treatment, where caste identities were not revealed, low-caste boys solved the mazes just as well as high-caste ones. Publicly revealing castes instead reduced the performance of the low-caste boys. And, once they were asked about the reasons for this performance drop, the low-caste boys answered that felt they could not or did not dare to excel and lessen the actions of the high-caste boys [Hoff and Pandey, 2014].

Mental models have been thoroughly studied by social psychologists who posit that they contextualise individuals' self-image, self-definition and self-evaluation [Markus and

\footnotetext{
${ }^{3}$ Alesina and Giuliano [2015] focus primarily on culture. They review a wide variety of definitions and posit that most empirical papers define culture as the set of values and beliefs that are passed down fairly unchanged over generations. Theoretical papers instead treat beliefs and values differently. Some scholars have defined culture as the beliefs about the consequences of one's action, others instead see culture as embodied in values and preferences. In this study, I rely on the notion of mental models. Mental models are the "templates" that individuals use to interpret events and they are transmitted from one generation to the next. Mental models affect the expectations of the results of one's action. As a consequence, this definition reconciles the empirical and theoretical definitions of culture but the focus is on the maps people use to interpret the world, rather than on (or in addition to) values, i.e. the ends of actions.
} 
Kitayama, 1991]. They shape people's perceptions on how things might or should work and be [Baumeister and Bushman, 2010]. Ajzen [1991] elaborates further and states that mental models affect people's perceived freedom to choose a behaviour and their sense of control over its outcome. Generally, one might explain human behaviour on the basis of individual volition. Nonetheless, some behaviours cannot be uniquely explained by volition. In some occasions, people carry out certain behaviours because they feel they are supposed to, and they have no choice of doing otherwise. Their volition is insufficient to defy mental models' prescriptions which are conceived as stringent and hard-to-eradicate. This consequently leads to low individual perceived behavioural control which Ajzen [2002] has defined as an individual belief that the performance of a behaviour is possible and under one's voluntary control. Perceived behavioural control affects the intentions and decisions to carry out certain actions. All else equal, high levels of perceived behavioural control intensify the intention to exert some degree of volitional control over performance and enhance effort and perseverance to defy the status quo [Ajzen, 2002]. ${ }^{4}$ Conversely, when people feel that their choices are dictated by mental models, their perceived behavioural control is low. This suffocates intentions and triggers apathy and conformism.

The extreme consequences of low perceived behavioural control have been analysed in an experimental fashion by Overmier and Seligman [1967] who, in their original experiment, observed that dogs repeatedly exposed to unavoidable electric shocks eventually ceased the effort to escape even when this was made possible. Dogs learned to be helpless as the shock was perceived as independent from their action, and this hindered their escape responses. Because of past experiences and beliefs concerning world functioning the dogs felt they did not have any control over their actions and thus, submissively accepted the impossibility to avoid the shocks. Numerous experiments confirmed that humans face learned helplessness as well [Hiroto and Seligman, 1975, Klein et al., 1976]. According to Rabow et al. [1983] in humans, learned helplessness is generated by the necessity to adapt to cultural and structural barriers to achievement, i.e. by stringent mental models which generate a sense of disempowerment, and low perceived behavioural control. Prolonged disempowerment convinces people that their choices as well as the

\footnotetext{
${ }^{4}$ Ajzen [2002] clarifies the difference between perceived behavioural control and self-efficacy. The author claims that despite the fact that the concept of perceived behavioural control owes its greatest debt to Bandura's self-efficacy, the two concepts are distinct. Self-efficacy is defined as the beliefs in one's capability to organise or carry out a certain action [Bandura, 1997]. This concept focuses on the control over the behaviour. Perceived behavioural control instead denotes the degree of control one is subjectively able to exercise over the performance of a behaviour. The focus here is on the control over the outcomes or events and not on the behaviour itself. Perceived behavioural control has a higher affinity with Bandura's definition of outcome expectancies. Bandura in fact distinguishes between selfefficacy, i.e. the perceived ability to perform an action, and outcome expectations, i.e. the perceived likelihood that carrying out a certain action will generate a given outcome [Bandura, 1997]. Perceived behavioural control is thus more similar to outcome expectancies rather than to self-efficacy.
} 
occurrence of possible negative outcomes escapes from their control. As a consequence, individuals will experience: (i) low motivation to change the status quo; (ii) frustration and resignation; (iii) lower ability to learn in similar situations [Seligman et al., 1979]; and (iv) lower well-being [Martinko and Gardner, 1982].

This literature shows that when people feel that their performance is dictated by hardto-eradicate mental models and their volition too weak to surpass mental models' prescriptions, choices can become path-dependent. Due to sense of scarce decisional power and control over the outcomes, people comply with what the mental model prescribes. Over time this spirals into higher reluctance in changing the status quo. In fact, the ability to recognise the possibility for improvement depends on individuals' perceived behavioural control and self-evaluations. If these are low, then any outcome is submissively accepted. As a consequence, individuals live in "equilibrium fictions" [Hoff and Stiglitz, 2010] wherein mental models are not challenged and thus reproduce.

According to Mantzavinos et al. [2004], the unquestionable reproduction of mental models affects the functioning and evolution of institutions. Institutional change reflects the evolution of mental models [Denzau and North, 1994]. In fact, according to North [1994, p.363] "the relation between mental models and institutions is an intimate one". While mental models are the internal representations that individual cognitive systems elaborate to portray the world's functioning, institutions are the external mechanisms individuals build to order and systematise the environment. Mental models lead to resolving shared problems, i.e. creating institutions, in a common way. And as institutions exhibit dynamic increasing returns, once solutions to problems are found, agents apply them each time similar problems arise. Thus, institutions evolve in a path-dependent manner.[Arthur, 1994, David, 1985]

Institutional path-dependence may ultimately affect the economic game leading to suboptimal results. Although institutions generally lead to low transaction costs, better ability to capture gains from trade and economic growth, this can fail to occur. The lack of improved institutional frameworks can hamper growth. In fact, while economic growth can occur in the short-run independently from the institutional structure in place [North, 2003], long-run and sustainable development requires, among other things, the development of rule of law and the expansion and protection of civil and political freedoms [Sen, 2001]. Social and economic arrangements, as well as political and civil rights, represent important determinants of countries' economic performance. But, cognitive and institutional path-dependence can ultimately induce economic path-dependence [Mantzavinos et al., 2004].

Summarising, economic performance is a consequence of, among other things, the institutional frameworks in place. These are in turn determined by the mental models 


\section{Mental models and Institutions}

individuals inter-subjectively share. Path-dependence in mental models leads to institutional path-dependence and at last to economic path-dependence.

This chapter sheds light on this chain of relations. In the first part of this chapter, I focus on the nexus between mental models and institutions. Subsequently I study the joint effect of mental models and institutions on economic growth. It is beyond the scope of this chapter to re-establish the role of institutions in the development process. A seminal paper by Rodrik et al. [2004] showed the primacy of institutions in determining countries' development paths, recently Acemoglu et al. [2014] show the key importance of democratisation. In this chapter I rather explore whether also mental models play a role.

\subsection{Data}

A key issue to carry out an empirical analysis to possibly establish a nexus between mental models, institutions and ultimately growth is how to measure mental models. It is very hard to measure mental models' malleability, but following the literature reviewed above, it is reasonable to believe that the one of the best ways to do so is to use a measure of perceived behavioural control as a proxy. In fact, as mentioned in section 4.2, mental models condition individual perceptions on whether actions are possible and under one's voluntary control.

Two different sources provide a good measure of perceived behavioural control. The first source is the European and World Value Surveys. These surveys question individuals on social and personal values and individual attributes. One of the available questions, namely question A173, reads as follows:

Some people feel they have completely free choice and control over their lives, while other people feel that what they do has no real effect on what happens to them. Please use this scale (1-10) where 1 means "no choice at all" and 10 means "a great deal of choice" to indicate how much freedom of choice and control you feel you have over the way your life turns out.

This question perfectly captures the hallmark components of perceived behavioural control, namely freedom of choice and control beliefs. The World and European value surveys (WVS-EVS) have been carried out since 1981. Data for question A173 is available for 103 countries. Not all countries went through all 6 available waves of the surveys. Table 4.8 in the Appendix reports the oldest and most recent available year, as well as the total number of available surveys waves for each country. ${ }^{5}$ As ours is a country level analysis,

${ }^{5}$ Data for the first wave were collected between 1981 and 1984, for the second wave between 1989 and 1993 . The third wave was conducted between 1994 and 1998, while the fourth between 1999 and 
we computed an unconditional average of the individual answers for each available wave. This provides an indication of the "nationally perceived sense of control".

The second alternative measure can be found in the Gallup Analytics World Poll survey data. These surveys question people's will on a wide variety of topics. Within the citizen engagement part of the survey, one of the questions reads:

In this country, are you satisfied or dissatisfied with your freedom to choose what you do with your life?

The data provide the percentage of respondents who are satisfied or unsatisfied with their freedom of choice. Again, this question well depicts the level of behavioural control people feel in their countries. This data has been collected from 2005/2006 to 2016 mostly by means of face-to-face or landline phone interviews in 163 countries. Table 4.8 in the Appendix reports the available year for each country. On average, 1083 respondents were surveyed in each country. Not all countries were surveyed every year within this timeperiod. ${ }^{6}$ Differently from the WVS, Gallup data analytics already provides an aggregate measure which is the share of the sample population which is satisfied with the freedom of choice it experiences. We use this as a country level measure for perceived behavioural control.

Both datasets present advantages and disadvantages. The World and European Value surveys question captures better than the Gallup World Poll question the hallmark components of perceived behavioural control. In fact, while the former focuses on both freedom of choice and control over outcomes, the latter limits its attention to freedom of choice. Conversely, Gallup World Polls surveys are more complete. The number of countries covered is higher but also the length of the time series per country is on average higher than the one available in the World and European Value surveys.

Despite their peculiarities, there is a positive correlation between the two available measures of perceived behavioural control. ${ }^{7}$ Figure 4.1 presents a scatter plot of the two measures. Countries appear multiple times if both measure are jointly available for multiple years.

It can be observed that countries with high freedom of choice according to the Gallup World Poll surveys are also those in which people feel free to choose and control the outcome of their actions according to the World Value Surveys. Nonetheless, I will carry

2004. The fifth and sixth wave took place respectively between 2005 and 2009, and between 2010 and 2014. Out of 103 countries, 21 were surveyed only once, 20 only twice, 19 only 3 times and 15 only 4 times. Only 28 countries underwent 5 or 6 waves.

${ }^{6}$ Out of 163 countries, 6 were surveyed only once, 4 only twice, 6 only 3 times, 11 only 4 times. Five countries were surveyed 5 times, and 6 were surveyed 6 times, 5 were surveyed 7 times. Ten countries underwent 8 waves, 24 underwent 9 surveys, 23 underwent 10 and 63 were surveyed for a total of 10 years.

${ }^{7}$ The correlation between the Gallup measure and the WVS one stands at 0.41 


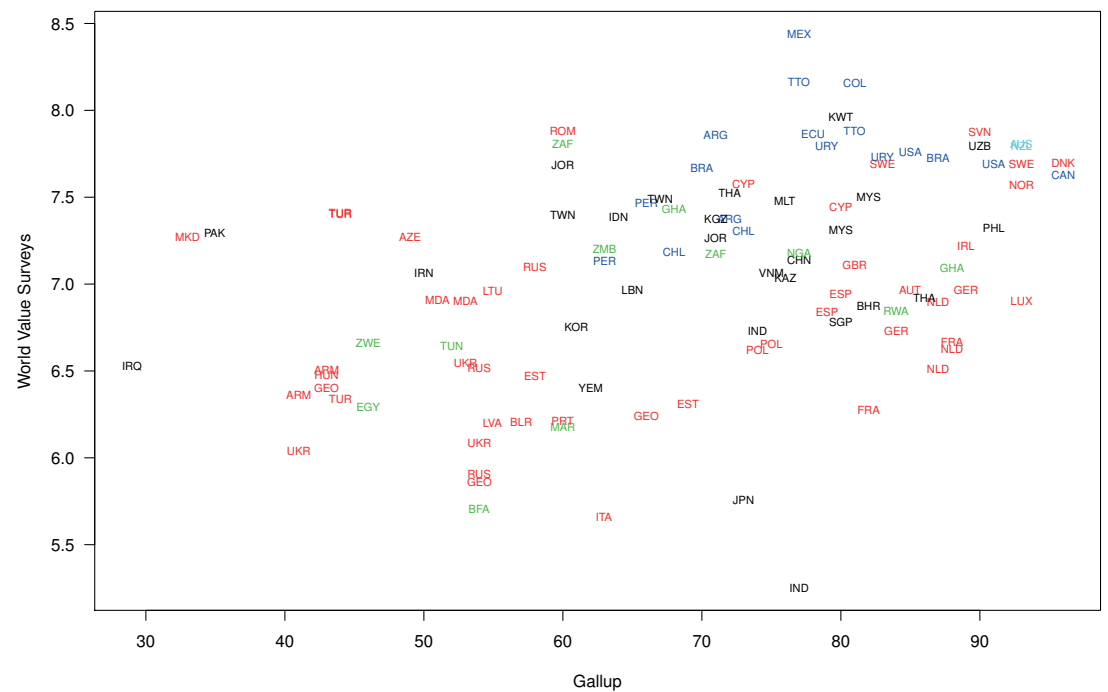

Figure 4.1: Gallup vs WVS

out the analysis considering both and I will identify them by the name Gallup and WVS respectively in the regression tables.

As a measure of institutions, I use one of the most common indicators in the literature, that is rule of law as elaborated by Kaufmann and Kraay [2002]. This indicator ranges between -2.5 and 2.5 and considers the level of property rights protection, contract enforcement, the functioning and independence of the judiciary, including the police, and the probability of crime and violence in a country. This data was retrieved from the World Bank World Governance Indicators (WGI), for the years 1996, 1998, 2000 and from 2002 until 2015. As an alternative measure, I also use the ICRG Quality of Government Indicator. This a well known indicator in the literature and is the mean value of the ICRG variables "Corruption" "Law and Order" and "Bureaucracy Quality". It ranges from 0 to 1 and is available from 1984 to 2015. These measures are also highly correlated, but I will present the main results of this chapter using both measures which will be identified by the acronym WGI and ICRG respectively in all subsequent figures and regression tables.

The main measure for economic performance is GDP per capita on a PPP basis. It was retrieved from the Penn World Tables mark 9.0. This data is available for a long time-span (1950-2014) and the specific year used in the empirical estimation is reported in the text or footnotes to each table. 


\subsection{Mental models and institutions}

After having argued that mental model malleability is well proxied by perceived behavioural control and having described the best available measures for it, in this section I describe the relation between mental models and institutions. I will first evaluate a pooling model, second I will control for country and time fixed effects.

\subsubsection{Pooling the data}

As a first step in this analysis, I plot over time the Gallup measure for perceived behavioural control and rule of law for (WGI) all countries for which both measures are available. It appears to exist relation between the 2 variables. Countries wherein people feel they have a high freedom of choice tend also to have higher rule of law. Nonetheless, some continent heterogeneity exists.

Thus, In order to properly assess the relation between mental model and rule of law, I run the following pooling model

$$
R o L_{i t}=\beta_{0}+\beta_{1} M M_{i t}+\epsilon_{i t}
$$

where $R o L_{i t}$ stands for the rule of law of country $i$ at a specific point in time and $M M_{i t}$ identifies degree of mental models malleability proxied by perceived behavioural control. This model allows me to evaluate whether a change in mental models corresponds to a change in the institutional structures in place. The results of this specification are reported in Table 4.1. In column (1-5) I use the WGI as a dependent variable to measure institutions, while in column (6-10) I rely on the ICRG data. As for mental models, I alternatively rely on both available measures which are here indicated by Gallup and WVS. 


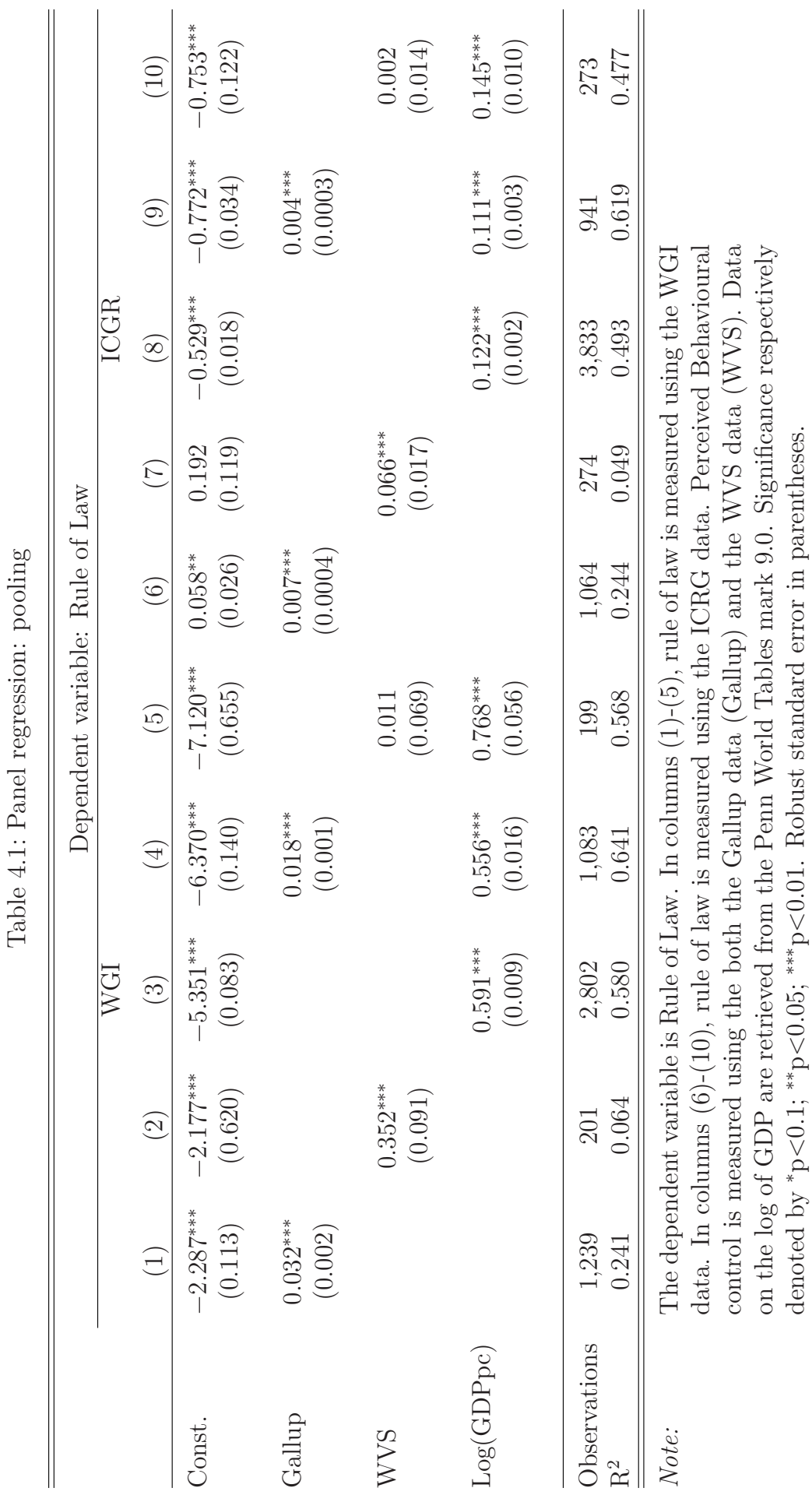


When using the WGI indicator for rule of law and the Gallup measure for perceived behavioural control, it can be seen that a ten percentage points increase in a country's freedom of choice will improve its rule of law by 0.32 , allowing the country to move from the rule of law of India or Tunisia to the rule of law of Italy in 2015. Given that the WGI measure for rule of law ranges between -2.5 to +2.5 , this is a considerable effect. When controlling for economic outcomes, the coefficient of behavioural control nearly halves but stays significant. This means that when controlling for per capita income, a ten percentage points increase in perceived behavioural control generates a increase of 0.18 in rule of law.

These results are also confirmed when using the ICGR dataset to measure institutions (see columns 6-10). In this case, a ten percentage points increase in freedom of choice improves rule of law by 0.07 or 0.04 when controlling for per capita income. As the country risk index is bounded between 0 and 1 this is not a negligible effect.

If we use the WVS data for perceived behavioural control and the WGI data for rule of law, the effect remains positive. In this case a one point increase in the perceived behavioural control, meaning shifting from most people in a country feeling not at all free and in control of their life to barely so, leads to an increase of rule of law of 0.3. This means that a one point increase in perceived behavioural control would lead a country from the rule of law of Chile which stood at 1.33 in 2015, to the rule of law of Iceland which was 1.67 in the same year. This effect remains significant even when using the ICRG data as a dependent variable, but its size reduces noticeably. In this case a one point increase in the WVS measure, leads to a 0.06 points increase in the ICRG rule of law. Conversely, controlling for income makes the coefficient non-significant (see column 10) but the coefficient's size remains stable. It is worth acknowledging that when using WVS data, the sample size drops significantly, thus one should be careful with the interpretation of these coefficients.

Controlling for geographical country characteristics, namely the country absolute latitude and longitude and whether or not the country is landlocked, does not alter the coefficients of mental models. Independently from the measure of institutions and perceived behavioural control used, the effect of mental models remains positive and significant for all model specifications. The detailed results are reported in Table 4.9 the Appendix.

Table 4.1 has shown that mental models and rule of law co-evolve on a cross-sectional basis. Nonetheless, it is worth exploring country differences and see whether in each country perceived behavioural control and rule of law consistently changed in the same direction. 


\subsubsection{Country and time fixed effect models}

An approximate idea of whether mental models affected countries' institutional structures, can be gathered plotting perceived behavioural control and rule of law over time for some specific countries. This is depicted in Figure 4.2 where the data for selected countries is shown. Uniquely for illustrative purpose, to draw this figure I selected some countries primarily looking at the annual rate of growth of the two variable of interest. I specifically selected those countries whose annual change in rule of law and perceived behavioural control was the highest. Venezuela and the Syrian Arab Republic are the two countries in which both rule of law and perceived behavioural control deteriorated at the fastest pace. Venezuela saw its rule of law declining of 1.11 points and peoples' satisfaction declined by 39 percentage points. In Syria, rule of law declined by 1.027 points and its citizens perceived behavioural control by 24 percentage points. In Liberia instead, people's perceived freedom of choice decreased by 4 percentage points, while rule of law improved by 1.35 points. New Zealand and Italy were chosen mostly to have an equal representation of all continents.

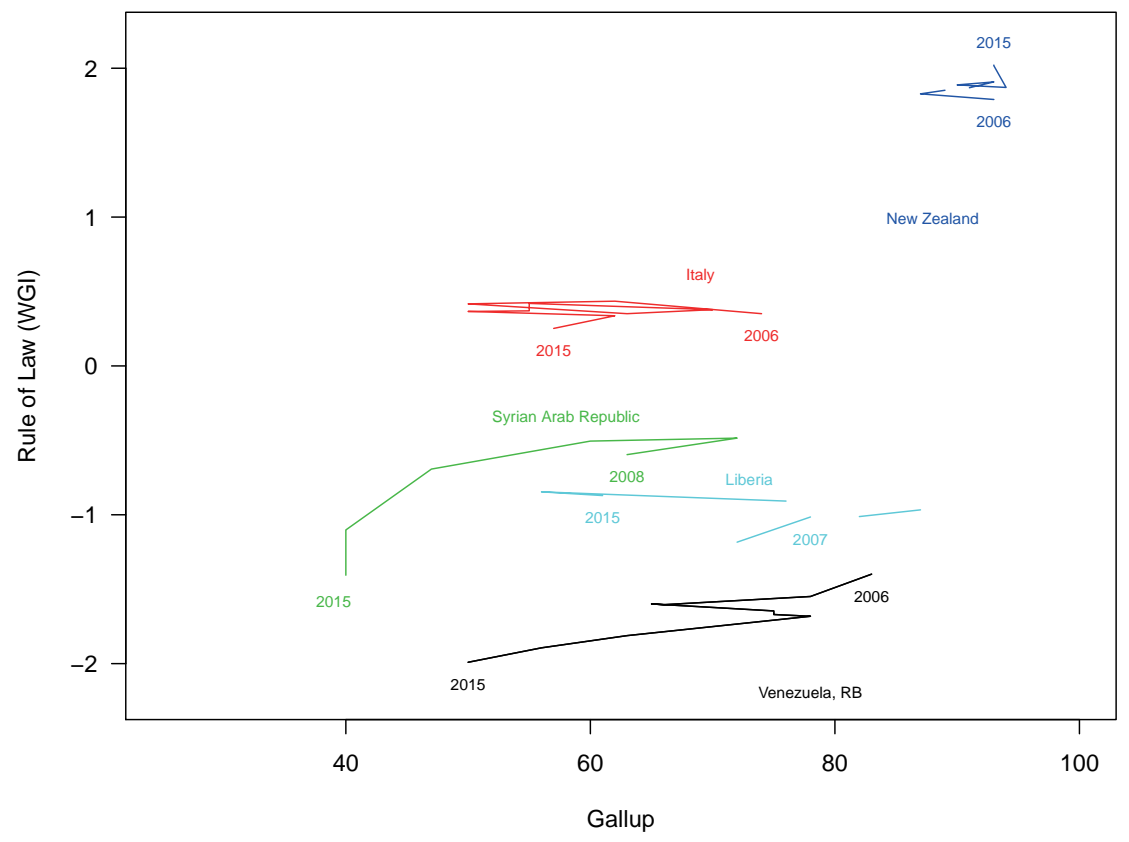

Figure 4.2: Rule of Law vs Gallup: selected countries

This plot clearly shows that, at country level, the relation between mental models and institutions is much weaker. Despite a possible increase (decrease) in freedom of choice, 
in some countries, there seems to be little change in rule of law. In order to quantify this effect, for all countries in my sample, I run the following model

$$
\operatorname{RoL}_{i t}=\beta_{0, i}+\beta_{1} M M_{i t}+\epsilon_{i t}
$$

where I include country fixed effects to remove the impact on rule of law of fixed country characteristics potentially correlated with mental models. Again, $R o L_{i t}$ is my dependent variable for rule of law and $M M_{i t}$ is the main regressor for mental models. The results of this model specification are reported in table 4.2. As in Table 1, in columns (1-5) I rely on the WGI data to measure institutions, while in columns (6-10) I use the ICRG data. 


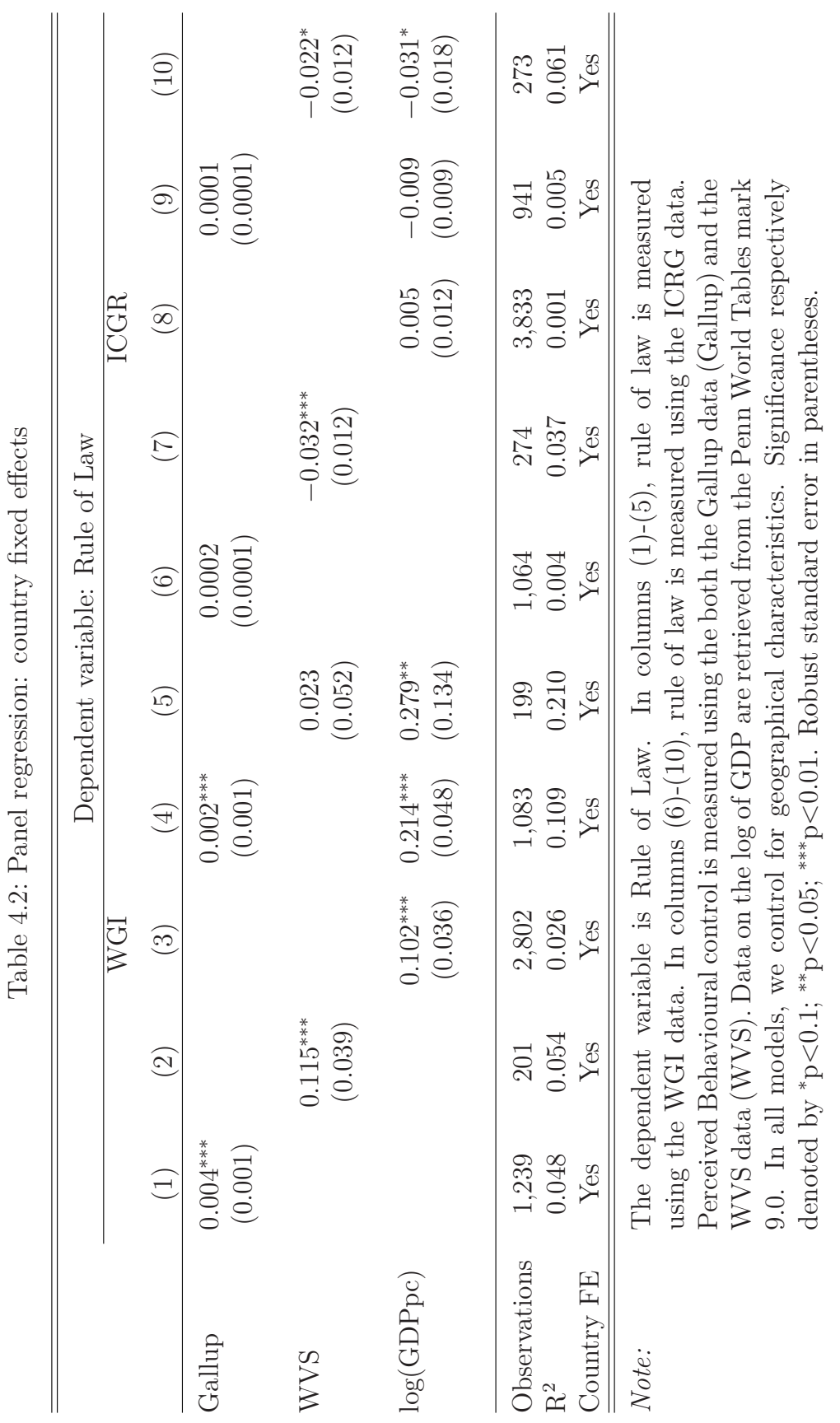


In general, it can be noticed that when controlling for country heterogeneity, the relation between mental models and institutions weakens by one order of magnitude with respect to the pooling regression presented in Table 4.1. In case perceived behavioural control is measured using the Gallup data and institutions are measured by means of the WGI data, a ten percentage points increase in people's perceived freedom of choice leads to a 0.04 increase in rule of law. This means that this increase in freedom of choice would allow the country to move from the rule of law of Botswana in 2015 to that of Uruguay in the same year. The coefficient halves when controlling for per capita income. If we use the ICRG measure for institutions, the effect of mental models becomes smaller and not significant. When instead mental models are proxied by the WVS data, and institutions are measure by the WGI rule of law measure, shifting from people feeling not at all to barely free or in control generates a 0.11 increase in rule of law. This effect is not significant when controlling for income, and even becomes negative when instead institutions are measured using the ICRG data. The sample size may play a important role in this.

I also carried out an estimation allowing for both country and time fixed effects. In this case the model estimated is

$$
R o L_{i t}=\beta_{0, i}+\beta_{1} M M_{i t}+\delta_{t}+\epsilon_{i t}
$$

The results are presented in Table 4.3. 


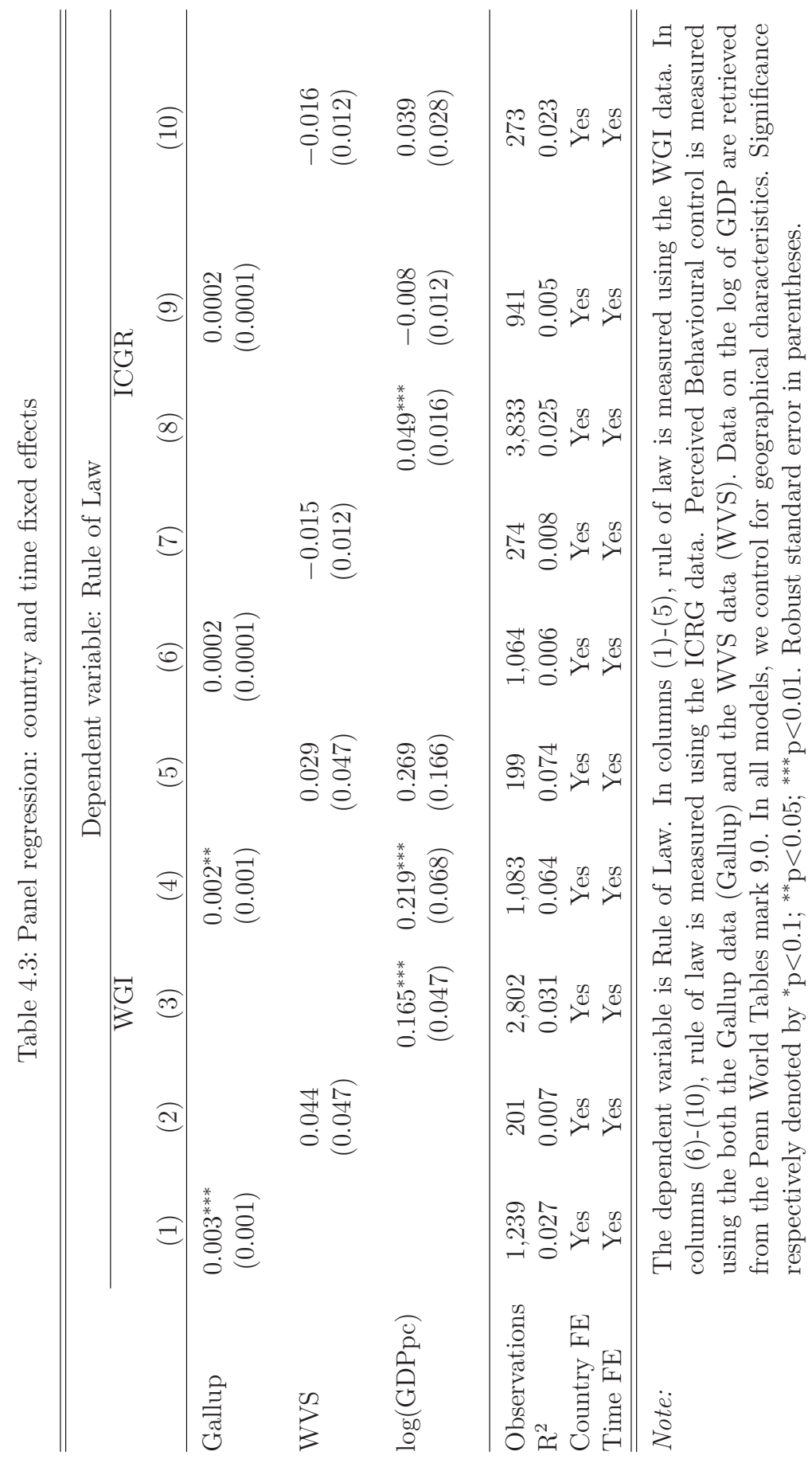


This table clearly shows that the coefficient for mental models proxied by the Gallup data, in spite of being small, is stable and significant when institutions are measured using the WGI indicator for rule of law. The effect disappears when we use the ICRG measure for institutions. If we use the WVS data, the effect of mental models on institutions disappears, no matter which of the two measure of institutions we refer to. Table 4.10 in the Appendix, show the first difference estimates. In this case, the coefficient for mental model is very small and not significant.

Summarising, available data suggest that mental models and institutions co-evolve. Most of the variation is nonetheless cross-sectional, not surprisingly. This relation weakens but stays significant when controlling for country fixed effects and measuring institutions via the WGI data.

\subsection{Mental models and growth}

According to the arguments exposed in section 4.2, countries wherein people perceive high freedom of choices and control should have better institutions and be wealthier. The importance of institutions on growth has been widely acknowledged. Conversely, very few study have been testing whether mental models play an independent role with respect to institutions on the path of growth of countries. Alesina and Giuliano [2015] advocate increased attention to both institutional and mental models. They claim that "we need to do more to fully understand their complementarities and how they jointly affect development" [Alesina and Giuliano, 2015, p.938]. In this second part of the chapter, I start from these premises and proceed using two different estimation techniques. First, I use panel data and estimate the effect of mental models and institutions on growth using a pooling model and a model which takes country and time fixed effects into account. Subsequently, I use cross-sectional instrumental variables regressions. There are in fact multiple reasons to move from a panel to a cross-sectional analysis. First, most of the effect that institutions and/ or mental models exert on growth is cross-sectional. This is a well known result in the literature [Acemoglu et al., 2014]. Moreover, several reverse causalities could be at work, as I argue at length in the following pages and this calls for the use of instrumental variable regressions. Ideally, one would like to have a time variant instrument to fully assess the chain of causal relations. Nonetheless, these types of instruments are hard to find. Recently, Acemoglu et al. [2014] rely on regional waves of democratisation as a exogenous source of variation in democracy levels. I, conversely, rely on time invariant instruments. Thus, a cross-sectional IV strategy is to be considered a first step in the attempt to uncover a possible independent effect of mental models and institutions on growth rates. 


\subsubsection{Pooling, country and time fixed effects models}

In order to provide an approximate idea on whether countries with highly malleable mental models are more affluent, I plot in Figure 4.3 my main variable for income and the Gallup measure of freedom of choice. In can be seen that a positive correlation between

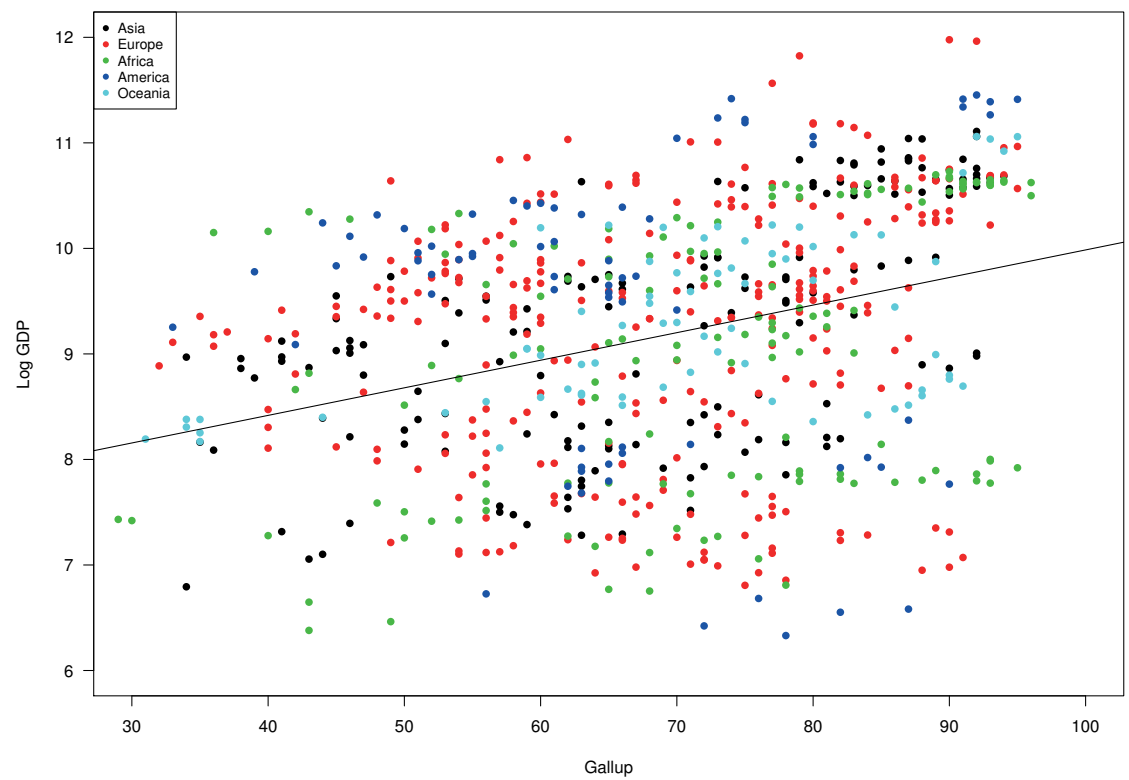

Figure 4.3: Log of Income per capita vs Gallup

the two variables exists, but, to properly assess the validity of this correlation I estimate the following pooling model

$$
y_{i t}=\beta_{0}+\beta_{1} M M_{i t}+\beta_{2} I N S_{i t}+\beta_{3} G E O_{i}+\varepsilon_{i}
$$

where $i$ indexes the countries analysed, $y_{i}$ is a measure of economic outcome at time $t$ such as $\log$ income per capita, $M M_{i t}$ represents my measure of mental model malleability at time $t, I N S_{i t}$ is a measure for institutions and $G E O_{i}$ identifies a common vector of time-independent geographical controls (latitude, longitude and a dummy variable for landlocked countries).

It is also worth exploring whether time and country fixed effects are relevant. For this purpose, I estimate the following model

$$
y_{i t}=\beta_{0, i}+\beta_{1} M M_{i t}+\beta_{2} I N S_{i t}+\delta_{t}+\varepsilon_{i t}
$$


wherein differently from above, $\beta_{0, i}$ denotes the full set of country fixed effects and $\delta_{t}$ indicates year fixed effects. The estimates of model 4.4 are presented in table 4.4 columns (1-3), whereas estimates for model 4.5 are presented in columns (4-6). In this table I use only the Gallup measure for mental models. 
Table 4.4: Panel regression: pooling and individual fixed effects

\begin{tabular}{|c|c|c|c|c|c|c|}
\hline & \multicolumn{6}{|c|}{ Dependent variable: Log(GDP per capita) } \\
\hline & \multicolumn{3}{|c|}{ Pooling } & \multicolumn{3}{|c|}{ Time and country fixed effext } \\
\hline & $(1)$ & $(2)$ & $(3)$ & $(4)$ & $(5)$ & (6) \\
\hline Const. & $\begin{array}{c}6.473^{* * *} \\
(0.124)\end{array}$ & $\begin{array}{c}8.243^{* * *} \\
(0.149)\end{array}$ & $\begin{array}{c}6.720^{* * *} \\
(0.128)\end{array}$ & & & \\
\hline Gallup & $\begin{array}{c}0.024^{* * *} \\
(0.002)\end{array}$ & $\begin{array}{c}0.005^{* * *} \\
(0.002)\end{array}$ & $\begin{array}{c}0.008^{* * *} \\
(0.002)\end{array}$ & $\begin{array}{c}0.002^{* * *} \\
(0.001)\end{array}$ & $\begin{array}{l}0.001^{* *} \\
(0.001)\end{array}$ & $\begin{array}{c}0.002^{* * *} \\
(0.001)\end{array}$ \\
\hline WGI & & $\begin{array}{c}0.597^{* * *} \\
(0.034)\end{array}$ & & & $\begin{array}{c}0.193^{* * *} \\
(0.055)\end{array}$ & \\
\hline Icrg & & & $\begin{array}{c}2.492^{* * *} \\
(0.183)\end{array}$ & & & $\begin{array}{c}-0.199 \\
(0.300)\end{array}$ \\
\hline Landlocked & $\begin{array}{c}-0.731^{* * *} \\
(0.063)\end{array}$ & $\begin{array}{c}-0.521^{* * *} \\
(0.058)\end{array}$ & $\begin{array}{c}-0.424^{* * *} \\
(0.065)\end{array}$ & & & \\
\hline Long. & $\begin{array}{c}-0.001 \\
(0.001)\end{array}$ & $\begin{array}{c}0.001^{*} \\
(0.001)\end{array}$ & $\begin{array}{c}0.001 \\
(0.001)\end{array}$ & & & \\
\hline Lat. & $\begin{array}{c}0.044^{* * *} \\
(0.001)\end{array}$ & $\begin{array}{c}0.024^{* * *} \\
(0.002)\end{array}$ & $\begin{array}{c}0.025^{* * *} \\
(0.002)\end{array}$ & & & \\
\hline Observations & 1,035 & 1,035 & 913 & 1,035 & 1,035 & 913 \\
\hline $\mathrm{R}^{2}$ (pooling) & 0.550 & 0.656 & 0.629 & & & \\
\hline $\mathrm{R}^{2}$ (within) & & & & 0.018 & 0.059 & 0.022 \\
\hline Country FE & & & & Yes & Yes & Yes \\
\hline Time FE & & & & Yes & Yes & Yes \\
\hline
\end{tabular}

Note: $\quad$ The dependent variable is $\log$ of GDP (at purchasing power parity) per capita from the Penn World Tables. In columns (1)-(3), we run a pooling model. In columns (4)-(6), I consider country and time fixed effects. Perceived Behavioural control is measured using the Gallup data. Institutions are measured using both the WGI and the ICRG data alternatively. In columns (1)-(3), we control for geographical characteristics. Significance respectively denoted by ${ }^{*} \mathrm{p}<0.1 ;{ }^{* *} \mathrm{p}<0.05 ;{ }^{* * *} \mathrm{p}<0.01$. Robust standard error in parentheses. 
Table 4.4 shows that mental models have an effect on per capita income growth. Most of the effect is cross-sectional as can be seen in columns (1-3). A ten percentage points increase in freedom of choice generates a 24 percent increase in income per capita. The coefficient decreases when controlling for institutional quality. In this case increasing perceived freedom by 10 percentage points leads to an income per capita increase of 5 (see column 2) or 8 log points (see column 3) depending how institutions are measured. This means that if in Venezuela in 2014 the share of individuals feeling in control of their life had been ten points higher, meaning $66 \%$ rather than $56 \%$, per capita income would have been 700 US dollars higher, reaching 14707 US dollars per year. When we allow for country and time fixed effects, the coefficient is smaller (see columns (4-6)). Specifically, if we do not control for institutional quality, a ten percentage points increase in freedom of choice generates a 2 percent increase in income per capita. Conversely, if institutional quality is accounted for, increasing freedom by 10 percentage points increases income per capita by 1 or 2 percent depending on the data used for institutions (see column 5 and 6 respectively). I estimated these models also using the WVS data as a proxy for mental models. The results are presented in Table 4.11 in the Appendix. Also in this case, when controlling for country and time fixed effects the effect of mental models on growth weakens and even becomes not significant mostly because of the reduced sample size.

The above reported relationships show that mental models have a positive effect on growth. But one should be careful to interpret these relations as they might suffer from endogeneity problems. In fact, there are several reasons to believe in the presence of an estimation bias.

Firstly, reverse causalities linking mental models, institutions and income could be in place. While geography is considered an exogenous variable that affects income per capita directly affecting agricultural productivity and morbidity, mental models and institutions are instead endogenous variables and reverse causalities are at work. Mental models affect income directly through possibly labor productivity for instance, but also indirectly through institutions. At the same time, higher mental models' malleability might be the result of increased domestic productivity and/or improved institutional structures rather than the cause thereof. Even in case of institutions reverse causality should be acknowledged. Although improvements in rule of law can directly affect income levels, it is also possible that higher quality institutions emerge as a consequence of higher income. At the same time, higher quality institutions might be the result of mental models' malleability. Secondly, there could be many omitted variables that affect economic performance and that might be naturally correlated with institutions and mental models. Moreover, ideally, in order to establish causality between mental models and growth, one would like to have a reliable measure of mental model's malleability from centuries 
ago to see how differences in past perceived behavioural control levels affected long-run development. Unfortunately, the best available measures of mental models malleability are recent and this poses some concerns of endogeneity.

To address these issues, in the next section, I use the instrumental variable framework.

\subsubsection{IV regressions}

In this section I run instrumental variable regressions to mitigate the reverse causalities mentioned above. Following previous literature, I have to rely on time independent instruments and this calls for the use of cross-sectional data.

More specifically, I employ the following basic econometric specification

$$
y_{i}=\beta_{0}+\beta_{1} M M_{i}+\beta_{2} I N S_{i}+\beta_{3} G E O_{i}+\varepsilon_{i}
$$

where $i$ indexes the countries analysed, $y_{i}$ is a measure of economic outcome namely log of per capita income in 2010, $M M_{i}$ represents my measure of mental model malleability, $I N S_{i}$ measure institutional quality and $G E O_{i}$ identifies a common vector of geographical controls. Differently from above, $M M_{i}$ and $I N S_{i}$ are considered endogenous variables and will be instrumented in the first stage as follows

$$
\begin{aligned}
& M M_{i}=\beta_{0}+\beta_{1} D I S T_{i}+\beta_{2} G E O_{i}+\varepsilon_{M M, i} \\
& I N S_{i}=\beta_{0}+\beta_{1} S E T T_{i}+\beta_{2} G E O_{i}+\varepsilon_{I N S, i}
\end{aligned}
$$

To measure institutions, I use the an average of the WGI rule of law over the period 1996-2009 as well as the average of protection against expropriation risk component from the ICRG dataset for the period 1985-2009. The latter is the same measure used by Gorodnichenko and Roland [2017] and Acemoglu et al. [2001] although, in their case, the average is calculated over a shorter time period (i.e.1985-2001). As for mental models I rely on the oldest available datapoint in both the Gallup and WVS data. In the text below, I will describe thoroughly only the case in which mental models are proxied using the Gallup data. Tables 4.14 and 4.16 in the Appendix present the results in case mental models are measured using the WVS data.

As an instrument for institutions, I will use the well-established settler mortality data [Acemoglu et al., 2001]. For what concerns mental models, a foolproof instrument is hard to find. I decided to rely on the geometric distance between the frequency of blood types in country $i$ and the frequency of blood types in a specific benchmark country as an instrument for mental models. This measure has been recently used by Gorodnichenko and Roland [2017]. In their paper, the geometric distance between the frequency of blood 
types in the country and the frequencies of blood types in the UK was used to instrument a measure of individualism and it proved to be a fairly strong instrument. They chose the UK as reference country because the United Kingdom is the most individualistic nation in their sample. Along the same reasoning, I use Norway as a benchmark country and construct the geometric distance between the frequency of blood types accordingly. Norway is, in my sample the country which over the period 2005-2016 has on average the highest level of perceived behavioural control, as measured by the Gallup data Poll. ${ }^{8}$ I used the data on frequency of blood types elaborated by Gorodnichenko and Roland [2017] and calculated the geometric genetic distance measure as follows

$$
\operatorname{Dist}_{i, N O R}=\left[\left(\bar{f}_{\text {blood } A, N O R}-\bar{f}_{\text {blood } A, i}\right)^{2}+\left(\bar{f}_{\text {blood }, N O R}-\bar{f}_{\text {blood }, i}\right)^{2}\right]^{1 / 2}
$$

where $\bar{f}_{\text {bloodA }}$ and $\bar{f}_{\text {bloodB }}$ denote the frequency of blood type $\mathrm{A}$ and $\mathrm{B}$ respectively, $i$ indicates the country, and NOR stands for the index for Norway the country from which the Euclidean distance is calculated. ${ }^{9}$

There are several reasons to believe that this could be a plausible instrument. First, both mental models and genes are vertically transmitted over generations of individuals. Parents pass down their genes as well as their world views. Thus, measures of genetic distance can be considered as a proxy for differences in mental models. To be clear, when using genetic distance as an instrument, I do not call for a causal relation between genes and mental models. I simply exploit the correlation between genetic distance and mental model differences across countries because both genes and world-views are transmitted from parents to children. Additionally, blood types are considered a neutral genetic marker. There is no evidence that the distribution of blood types affect countries' wealth [Gorodnichenko and Roland, 2017]. Moreover, it is also unlikely that economic development affects genetic pools in a short time-span. Thus, this measure is very likely to satisfy the exclusion restriction.

The use of genetic distance is not new in this literature. As mentioned above, my instrument, and its elaboration, is clearly inspired by Gorodnichenko and Roland's work [2017]. They interpret the genetic distance as a proxy for a specific cultural trait that is the degree of individualism within societies, which in their view is a source of a potential

\footnotetext{
${ }^{8}$ As a robustness check (not shown), I also calculated the genetic distance of all countries using Mexico as a benchmark. Mexico was chosen because this is the country which has the highest average perceived behavioural control measured using the WVS. Results do not seem to change.

${ }^{9}$ Refer to Gorodnichenko and Roland [2017] for a detailed discussion on the measure and the various sources. Although, Gorodnichenko and Roland [2017] do not mention this, I believe that the genetic distance measure is calculated using blood types $\mathrm{A}$ and be due to data availability. In fact, group 0 and $\mathrm{AB}$ are rare. In the Appendix I show that the results are stable even when we calculate the distance between the frequency of blood types in country $i$ with respect to the frequency of blood types in the UK as Gorodnichenko and Roland [2017] did.
} 
bias distorting people's propensity to strive for social status, personal achievement and innovation. Recalling Max Weber's view on the protestant ethic of Calvinism, they claim that the pursuit of one's profession and status in society is a powerful force behind development. They use the well-established indicator elaborated by Hofstede [2003] to measure individualism. Theirs is mainly a cross-sectional analysis. My study instead focuses on mental models and I rather use genetic distance as a proxy for mental models' malleability and claim that the perceived capacity to freely choose and control life's outcomes is likely to trigger motivation for economic effort. Genetic measures have been also used by Guiso et al. [2009] and Spolaore and Wacziarg [2009]. Guiso et al. [2009] use genetic distance as to proxy cultural and genetic dissimilarity which affects trust levels and engagement in trade activities in societies. Spolaore and Wacziarg [2009] consider genetic distance as an obstacle to technology diffusion because distance impedes communication and thus populations that are far from one another will have more difficulty communicating and thus benefit less from innovation.

Table 4.5 presents the OLS and IV estimates. The dependent variable is log income per capita in 2010, whereas mental models are proxied using the oldest available data point in the Gallup dataset and institutions are measured by an average of protection against expropriation risk component from the ICRG dataset. All variables have been standardised because metric-free coefficients can be easily compared, they are often used in these types of growth regressions [Rodrik et al., 2004], and last they seem to give better results.

Columns (1-3) present the basic OLS estimates for the full sample. As can be seen in column (1) the coefficient for mental models measured by means of the oldest available Gallup data is positive and significant. Specifically, increasing individuals freedom of choice by 13.54 points, i.e. one standard deviation, leads to an increase in log income of 0.55 (i.e. $0.47^{*} 1.176$ which is $\beta$ times the standard deviation of income). This effect disappears when, in column (3), institutions and geographical variables are added. In column (4) and (5) I instrument the Gallup data on mental models by the genetic distance from Norway. In column (5), I also control for institutional quality using the ICRG data on expropriation risks and property rights protection. When instrumented, the coefficient for mental models becomes twice as large as the one obtained with the OLS and stays significant. In these cases, one standard deviation increase in freedom of choice generates approximately one log point increase in income per capita. Once mental models are instrumented and institutions and geography are accounted for (see columns(5)), the coefficient of mental models declines but stays positive and significant. Thanks to this model specification we can explain 47 percent of the variation of per capita income. The geometric distance between the frequency of blood types in country $i$ and the frequency of 
blood types in Norway proves to be a strong instrument as shown by the high first stage F-statistics (refer to Table 4.12 in the Appendix for details on the first stage regressions). In column (6), both mental models and institutions are instrumented. In this case, none of the regressors is significant but their size is relatively stable. It is worth mentioning that this model exhibits a very poor fit to the data as shown by the negative $R^{2}$ obtained.

Table 4.5: Relative effects of institutions (Icrg) and mental models (Gallup) on economic development

\begin{tabular}{|c|c|c|c|c|c|c|}
\hline & \multicolumn{6}{|c|}{ Dependent variable: log income 2010} \\
\hline & \multicolumn{3}{|c|}{ OLS } & \multicolumn{2}{|c|}{ IV+controlling inst } & \multirow{2}{*}{$\begin{array}{l}\text { IV } \\
(6)\end{array}$} \\
\hline & (1) & $(2)$ & (3) & (4) & (5) & \\
\hline Const. & $\begin{array}{l}-0.000 \\
(0.080)\end{array}$ & $\begin{array}{l}-0.000 \\
(0.057)\end{array}$ & $\begin{array}{c}0.046 \\
(0.054)\end{array}$ & $\begin{array}{c}-0.389^{* * *} \\
(0.108)\end{array}$ & $\begin{array}{l}-0.158 \\
(0.105)\end{array}$ & $\begin{array}{l}-0.130 \\
(0.157)\end{array}$ \\
\hline Gallup & $\begin{array}{c}0.471^{* * *} \\
(0.070)\end{array}$ & & $\begin{array}{c}0.098 \\
(0.062)\end{array}$ & $\begin{array}{c}1.074^{* * *} \\
(0.203)\end{array}$ & $\begin{array}{c}0.780^{* * *} \\
(0.204)\end{array}$ & $\begin{array}{c}0.520 \\
(0.398)\end{array}$ \\
\hline Icrg & & $\begin{array}{c}0.779^{* * *} \\
(0.057)\end{array}$ & $\begin{array}{c}0.544^{* * *} \\
(0.070)\end{array}$ & & $\begin{array}{l}0.318^{* *} \\
(0.158)\end{array}$ & $\begin{array}{c}1.505 \\
(1.380)\end{array}$ \\
\hline Lat. & & & $\begin{array}{c}0.375^{* * *} \\
(0.052)\end{array}$ & & $\begin{array}{l}0.128 \\
(0.131)\end{array}$ & $\begin{array}{l}-0.348 \\
(0.594)\end{array}$ \\
\hline Long. & & & $\begin{array}{c}-0.002 \\
(0.042)\end{array}$ & & $\begin{array}{l}-0.018 \\
(0.070)\end{array}$ & $\begin{array}{l}-0.052 \\
(0.111)\end{array}$ \\
\hline Landlocked & & & $\begin{array}{c}-0.249^{*} \\
(0.133)\end{array}$ & & $\begin{array}{l}-0.500 \\
(0.335)\end{array}$ & $\begin{array}{l}-0.398 \\
(0.349)\end{array}$ \\
\hline $\begin{array}{l}\text { 1st stage F-Stat. (Gallup) } \\
\text { 1st stage F-Stat. (Icrg) }\end{array}$ & & & & 23.92 & 36.03 & $\begin{array}{c}16.79 \\
1.68\end{array}$ \\
\hline Observations & 120 & 120 & 120 & 54 & 54 & 54 \\
\hline $\mathrm{R}^{2}$ & 0.222 & 0.607 & 0.723 & 0.158 & 0.478 & -0.081 \\
\hline Residual Std. Error & 0.886 & 0.630 & 0.538 & 0.812 & 0.665 & 0.958 \\
\hline
\end{tabular}

Note:

The dependent variable in the second stage is log income (at purchasing power parity) per capita in 2010 from the Penn World Tables. Perceived Behavioural control is measured using the Gallup data. Institutions are measure by the ICRG data. In columns (4) and (6), Perceived Behavioural control is instrumented using the geometric distance of frequency of blood types A and B in a given country relative to the frequency of blood types A and B in Norway. In column (6), institutions are instrumented using settler mortality data. Latitude, longitude and a dummy variable for landlocked countries is used as controls in columns (3), (5), (6). All coefficients are standardised. Significance respectively denoted by ${ }^{*} \mathrm{p}<0.1 ;{ }^{* *} \mathrm{p}<0.05 ;{ }^{* * *} \mathrm{p}<0.01$. Robust standard error in parentheses. 
These results are confirmed when the same model specifications are estimated using the WGI data for institutional quality. These estimates are presented in Table 4.6. Surprisingly, even when using the WGI rule of law measure, a one standard deviation increase in perceived freedom, increases income per capita by 0.5 log points. The effect of mental models on growth increases in case I use genetic distance as and instrument and control for institutional quality (see column(5)). The results presented in column (6), when both mental models and institutions are instrumented, are inconclusive. In Table 4.14 and table 4.16 in the Appendix, I estimate the same models but using the WVS data for mental models. Also, in the Appendix I present the details of the first stage estimations. There is no remarkable difference between these results and those presented above. Nonetheless, it is worth mentioning that the genetic distance between the frequency of blood types is a very poor instrument for the WVS data. This has most probably to do with the fact that the sample size is much smaller. There are only 33 or 34 countries for which both the WVS and the settler mortality data are available. 
Table 4.6: Relative effects of institutions (WGI) and mental models (Gallup) on economic development

\begin{tabular}{|c|c|c|c|c|c|c|}
\hline & \multicolumn{6}{|c|}{ Dependent variable: log income 2010} \\
\hline & \multicolumn{3}{|c|}{ OLS } & \multicolumn{2}{|c|}{ IV+controlling inst } & \multirow{2}{*}{$\begin{array}{l}\text { IV } \\
(6) \\
\end{array}$} \\
\hline & $(1)$ & $(2)$ & $(3)$ & $(4)$ & $(5)$ & \\
\hline Const. & $\begin{array}{c}0.000 \\
(0.074)\end{array}$ & $\begin{array}{c}0.000 \\
(0.054)\end{array}$ & $\begin{array}{l}0.093^{*} \\
(0.054)\end{array}$ & $\begin{array}{c}-0.368^{* * *} \\
(0.102)\end{array}$ & $\begin{array}{l}-0.107 \\
(0.104)\end{array}$ & $\begin{array}{l}-0.118 \\
(0.138)\end{array}$ \\
\hline Gallup & $\begin{array}{c}0.467^{* * *} \\
(0.070)\end{array}$ & & $\begin{array}{c}0.066 \\
(0.067)\end{array}$ & $\begin{array}{c}1.110^{* * *} \\
(0.199)\end{array}$ & $\begin{array}{l}0.881^{* *} \\
(0.375)\end{array}$ & $\begin{array}{c}0.267 \\
(0.438)\end{array}$ \\
\hline WGI & & $\begin{array}{c}0.763^{* * * *} \\
(0.045)\end{array}$ & $\begin{array}{c}0.514^{* * * *} \\
(0.083)\end{array}$ & & $\begin{array}{c}0.003 \\
(0.311)\end{array}$ & $\begin{array}{c}0.895 \\
(0.569)\end{array}$ \\
\hline lat. & & & $\begin{array}{c}0.337^{* * *} \\
(0.061)\end{array}$ & & $\begin{array}{c}0.276 \\
(0.192)\end{array}$ & $\begin{array}{l}-0.170 \\
(0.320)\end{array}$ \\
\hline long. & & & $\begin{array}{c}0.043 \\
(0.042)\end{array}$ & & $\begin{array}{l}-0.007 \\
(0.082)\end{array}$ & $\begin{array}{l}-0.003 \\
(0.080)\end{array}$ \\
\hline landlocked & & & $\begin{array}{c}-0.381^{* * *} \\
(0.119)\end{array}$ & & $\begin{array}{l}-0.475 \\
(0.379)\end{array}$ & $\begin{array}{c}-0.689^{* *} \\
(0.331)\end{array}$ \\
\hline $\begin{array}{l}\text { 1st stage F-Stat. (Gallup) } \\
\text { 1st stage F-Stat. (WGI) }\end{array}$ & & & & 27.2 & 14.78 & $\begin{array}{c}18.28 \\
7.89\end{array}$ \\
\hline $\begin{array}{l}\text { Ist stage F-stat. (WGI) } \\
\text { Observations }\end{array}$ & 143 & 143 & 143 & 56 & 56 & $\begin{array}{l}7.89 \\
56\end{array}$ \\
\hline $\mathrm{R}^{2}$ & 0.218 & 0.582 & 0.672 & 0.233 & 0.430 & 0.411 \\
\hline Residual Std. Error & 0.887 & 0.649 & 0.583 & 0.771 & 0.691 & 0.702 \\
\hline
\end{tabular}

Note:

The dependent variable in the second stage is log income (at purchasing power parity) per capita in 2010 from the Penn World Tables. Perceived Behavioural control is measured using the Gallup data. Institutions are measured by the WGI data. In columns (4) and (6), Perceived Behavioural control is instrumented using the geometric distance of frequency of blood types A and B in a given country relative to the frequency of blood types A and B in Norway. In column (6), institutions are instrumented using settler mortality data. Latitude, longitude and a dummy variable for landlocked countries are used as controls in columns (3), (5), (6). All coefficients are standardised. Significance respectively denoted by ${ }^{*} \mathrm{p}<0.1 ;{ }^{* *} \mathrm{p}<0.05 ;{ }^{* * *} \mathrm{p}<0.01$. Robust standard error in parentheses.

I also run the same model specifications using the log of income per worker in 2000 as a dependent variable. This is the main dependent variable used by Gorodnichenko and Roland [2017] and Rodrik et al. [2004]. The main results are confirmed (see Tables 4.18, 4.19, 4.20 and 4.21 in the Appendix). According to the OLS specification, mental models have a positive and significant effect on growth. But, when mental models are instrumented by the genetic distance between blood types using Norway as the reference country and at the same time the institutional measure is instrumented by settler mortality, mental models exert no significant influence on growth. As pointed above, the evidence suggests that genetic distance is not a good instrument for the World Value Survey data. 


\section{Mental models and Institutions}

As an additional robustness check, I also used the geometric distance between the frequency of blood types using the UK rather than Norway as the benchmark country. This is the exact same instrument used by Gorodnichenko and Roland [2017]. The results are qualitatively the same as those presented above. They are presented in Tables 4.22 to 4.25 in the Appendix.

To conclude, in this section, I tried to mitigate the reverse causalities linking mental models, institutions and income growth. Due to the lack of well-suited time variant instruments, I used cross-sectional regressions. I provided weak evidence that suggests that mental models' malleability has a positive effect on growth. Some caveats in fact apply. Firstly, the instrument used for mental models proves quite strong when we use the Gallup data, but not when the WVS measure is used. This might have mainly to do with imperfect overlap between coverage of settler mortality and WVS data. Second, the effect of mental models is rather sensitive to including institutional quality measures in the OLS regression. And, when both my main regressors for institutions and mental models are instrumented, the effect of mental models on growth looses significance in most specifications but it keeps being positive and of consistent magnitude. Institutions seem to encompass most of the effect that mental models exert on growth. This is not entirely surprisingly given that institutions and mental models co-evolve as shown in the first part of this study using panel data. These coefficients should, nonetheless, be interpreted with care given the small sample size. Future research should re-assess these results when more data become available.

\subsection{Conclusions}

In this study, I first assess the co-evolution of mental models and institutions. This is the first study which aims to test empirically within this framework whether, as claimed by North [1994], Denzau and North [1994] and Mantzavinos et al. [2004], a "intimate" relation between mental models' stickiness and institutions exists. I test this hypothesis using cross-country and micro-level data. The evidence shows a positive relationship between mental models and institutions. Nonetheless, most of the variation is crosssectional. When controlling for country fixed effects I show that a ten percentage points increase in perceived freedom of control leads to a 0.04 points increase in rule of law, as measured in the WGI. This would allow a country like Botswana to reach the rule of law of Uruguay, for instance.

In the second part of the chapter, following Alesina and Giuliano's (2015) suggestion, I instead explore whether mental models can affect economic growth independently from institutions. A pooling model shows that mental models matter for growth and their 
effect is independent from institutions. This effect declines when allowing for country and time fixed effects. In order to tackle possible endogeneity issues, I use cross-sectional IV models and present some weak evidence on the fact that mental models independently affect growth. The estimates obtained are to be interpreted with care due to the imperfect overlap between the coverage of the main regressors and their instruments.

This study is not exempt from possible pitfalls. The first issue relates to the scarcity of the data available. The findings of this chapter should be re-assessed when more data become available. Secondly, I operationalised the measurement of mental models using a specific introspective belief which is perceived behavioural control. It is nevertheless worth acknowledging that mental models also act upon and mold other self-evaluation concepts. A wide range of possible replication is possible along these lines. Third, in this study I relied on time invariant instruments. But it should be acknowledged that time variant instruments and thus panel IV strategies would be ideal to properly assess the presence of a causal relation between mental models and growth.

Nonetheless, I believe this study is amongst the firsts to stress the role that mental models, through their influence on introspective beliefs, play on evolution of institutions and countries' growth. And, it paves the way towards a richer view of institutional change and ultimately economic development. It is well known that Sen's capability theory stresses that an increase in economic and political freedoms benefits everyone. Development is thus considered as a path towards the expansion of the economic and political freedoms individuals can enjoy [Sen, 2001]. Social psychologists, additionally, argue that in order to be able to enjoy those freedoms, individuals should not only be given the appropriate means but also have specific expectations and perceptions concerning the outcome of their choices. This chapter combines these ideas and shows the importance of mental models in defining the salience of certain beliefs and thus individuals' sense of control and freedom of choice. I show some evidence that mental models and the 'rules of the game' co-evolve. If people perceive little freedom in their lives, they will continue to think about the world and their role in it in static fashion [Fehr and Hoff, 2011]. They will not change their behaviour and institutions will not ameliorate. Conversely, increasing people's perceived freedom is likely to prompt a change in the quality of institutions. Ultimately, mental models might condition economic development. This study provides some evidence which suggests that, for a country to be wealthier, an increase in rule of law is important but at the same time an expansion in its citizens perception of control and freedom of choice should be promoted. 


\subsection{Appendix: Additional information and robustness checks}

Table 4.7: Variable and related sources

\begin{tabular}{|c|c|}
\hline Variable & Source \\
\hline Mental model WVS & World Value Surveys \\
\hline Mental models Gallup & Gallup Analytics \\
\hline Rule of Law WGI & Gorld Governance Indicators \\
\hline Rule of Law ICRG & International Country Risk Guide (ICRG) \\
\hline $\begin{array}{c}\text { Protection against expropriation } \\
\text { risk (ICRG) }\end{array}$ & Penn World Tables (PWT) mark 9.0 \\
\hline GDP per capita & Gorodnichenko and Roland 2017 \\
\hline $\begin{array}{c}\text { Frequencies of blood types } \\
\text { between each country and Norway) }\end{array}$ & \\
\hline
\end{tabular}


Table 4.8: Data for perceived behavioural control: available years

GALLUP

wVs

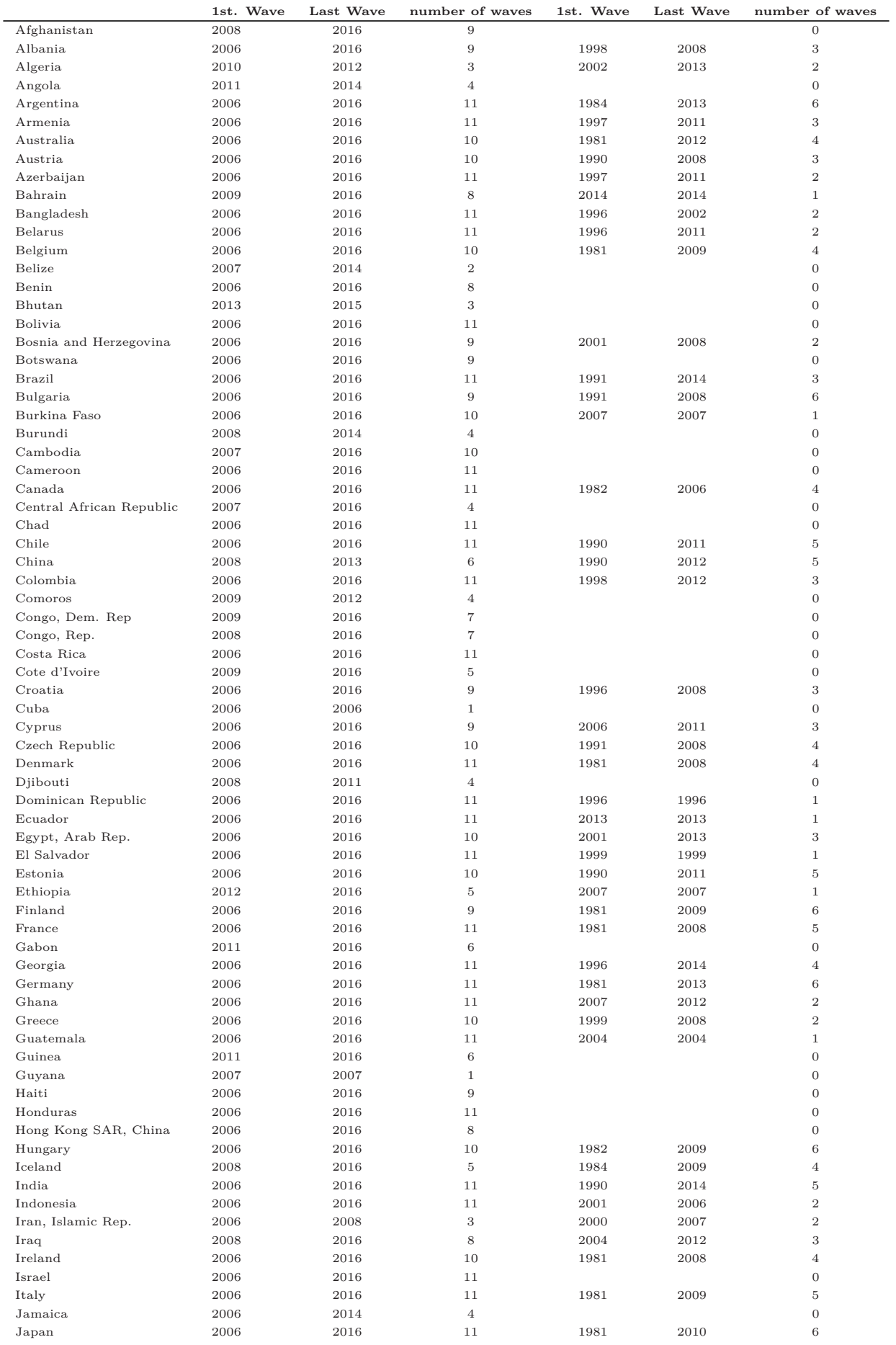




\begin{tabular}{|c|c|c|c|c|c|c|}
\hline Jordan & 2007 & 2016 & 9 & 2001 & 2014 & 3 \\
\hline Kazakhstan & 2006 & 2016 & 11 & 2011 & 2011 & 1 \\
\hline Kenya & 2006 & 2016 & 11 & & & 0 \\
\hline Korea, Rep. & 2006 & 2016 & 11 & 1982 & 2010 & 5 \\
\hline Kosovo & 2006 & 2016 & 9 & & & 0 \\
\hline Kuwait & 2006 & 2016 & 9 & 2014 & 2014 & 1 \\
\hline Kyrgyz Republic & 2006 & 2016 & 11 & 2003 & 2011 & 2 \\
\hline Lao PDR & 2006 & 2011 & 4 & & & 0 \\
\hline Latvia & 2006 & 2016 & 10 & 1990 & 2008 & 4 \\
\hline Lebanon & 2006 & 2016 & 11 & 2013 & 2013 & 1 \\
\hline Lesotho & 2011 & 2016 & 2 & & & 0 \\
\hline Liberia & 2007 & 2016 & 8 & & & 0 \\
\hline Libya & 2012 & 2016 & 3 & 2014 & 2014 & 1 \\
\hline Lithuania & 2006 & 2016 & 11 & 1990 & 2008 & 4 \\
\hline Luxembourg & 2008 & 2016 & 8 & 1999 & 2008 & 2 \\
\hline Macedonia, FYR & 2006 & 2016 & 10 & 1998 & 2008 & 3 \\
\hline Malawi & 2006 & 2016 & 9 & & & 0 \\
\hline Malaysia & 2006 & 2015 & 10 & 2006 & 2012 & 2 \\
\hline Mali & 2006 & 2016 & 10 & 2007 & 2007 & 1 \\
\hline Malta & 2008 & 2016 & 8 & 1983 & 2008 & 4 \\
\hline Mauritania & 2007 & 2016 & 10 & & & 0 \\
\hline Mauritius & 2011 & 2016 & 3 & & & 0 \\
\hline Mexico & 2006 & 2016 & 11 & 1981 & 2012 & 6 \\
\hline Moldova & 2006 & 2016 & 11 & 1996 & 2008 & 4 \\
\hline Mongolia & 2007 & 2016 & 9 & & & 0 \\
\hline Montenegro & 2006 & 2016 & 9 & 1996 & 2008 & 3 \\
\hline Morocco & 2010 & 2016 & 7 & 2001 & 2011 & 3 \\
\hline Mozambique & 2006 & 2015 & 5 & & & 0 \\
\hline Myanmar & 2012 & 2016 & 4 & & & 0 \\
\hline Namibia & 2007 & 2014 & 2 & & & 0 \\
\hline Nepal & 2006 & 2016 & 11 & & & 0 \\
\hline Netherlands & 2006 & 2016 & 10 & 1981 & 2012 & 6 \\
\hline New Zealand & 2006 & 2016 & 10 & 1998 & 2011 & 3 \\
\hline Nicaragua & 2006 & 2016 & 11 & & & 0 \\
\hline Niger & 2006 & 2016 & 11 & & & 0 \\
\hline Nigeria & 2006 & 2016 & 11 & 1990 & 2011 & 4 \\
\hline Norway & 2006 & 2016 & 6 & 1982 & 2008 & 5 \\
\hline Oman & 2011 & 2011 & 1 & & & 0 \\
\hline Pakistan & 2006 & 2016 & 11 & 2001 & 2012 & 2 \\
\hline Panama & 2006 & 2016 & 11 & & & 0 \\
\hline Paraguay & 2006 & 2016 & 11 & & & 0 \\
\hline Peru & 2006 & 2016 & 11 & 1996 & 2012 & 4 \\
\hline Philippines & 2006 & 2016 & 11 & 1996 & 2012 & 3 \\
\hline Poland & 2006 & 2016 & 11 & 1989 & 2012 & 6 \\
\hline Portugal & 2006 & 2016 & 10 & 1990 & 2008 & 3 \\
\hline Puerto Rico & 2006 & 2014 & 2 & & & 0 \\
\hline Qatar & 2008 & 2012 & 4 & 2010 & 2010 & 1 \\
\hline Romania & 2006 & 2016 & 10 & 1993 & 2012 & 6 \\
\hline Russian Federation & 2006 & 2016 & 11 & 1990 & 2011 & 6 \\
\hline Rwanda & 2006 & 2016 & 9 & 2007 & 2012 & 2 \\
\hline Saudi Arabia & 2007 & 2016 & 9 & 2003 & 2003 & 1 \\
\hline Senegal & 2006 & 2016 & 11 & & & 0 \\
\hline Serbia & 2006 & 2016 & 9 & 1996 & 2008 & 3 \\
\hline Sierra Leone & 2006 & 2016 & 9 & & & 0 \\
\hline Singapore & 2006 & 2016 & 10 & 2002 & 2012 & 2 \\
\hline Slovak Republic & 2006 & 2016 & 8 & 1990 & 2008 & 5 \\
\hline Slovenia & 2006 & 2016 & 9 & 1992 & 2011 & 6 \\
\hline Somalia & 2014 & 2016 & 3 & & & 0 \\
\hline South Africa & 2006 & 2016 & 11 & 1982 & 2013 & 6 \\
\hline Spain & 2006 & 2016 & 11 & 1981 & 2011 & 6 \\
\hline Sri Lanka & 2006 & 2015 & 10 & & & 0 \\
\hline Sudan & 2009 & 2016 & 7 & & & 0 \\
\hline Suriname & 2012 & 2012 & 1 & & & 0 \\
\hline Swaziland & 2011 & 2011 & 1 & & & 0 \\
\hline Sweden & 2006 & 2016 & 11 & 1982 & 2011 & 6 \\
\hline Switzerland & 2006 & 2016 & 6 & 1989 & 2008 & 4 \\
\hline Syrian Arab Republic & 2008 & 2015 & 7 & & & 0 \\
\hline Taiwan, China & 2006 & 2016 & 9 & 1994 & 2012 & 3 \\
\hline Tajikistan & 2006 & 2016 & 11 & & & 0 \\
\hline Tanzania & 2006 & 2016 & 11 & 2001 & 2001 & 1 \\
\hline Thailand & 2006 & 2016 & 11 & 2007 & 2013 & 2 \\
\hline Togo & 2006 & 2016 & 6 & & & 0 \\
\hline Trinidad and Tobago & 2006 & 2013 & 4 & 2006 & 2011 & 2 \\
\hline Tunisia & 2009 & 2016 & 8 & 2013 & 2013 & 1 \\
\hline Turkey & 2006 & 2016 & 11 & 1996 & 2011 & 5 \\
\hline Turkmenistan & 2012 & 2016 & 5 & & & \\
\hline
\end{tabular}


4.7 Appendix: Additional information and robustness checks

\begin{tabular}{|c|c|c|c|c|c|c|}
\hline Uganda & 2006 & 2016 & 11 & 2001 & 2001 & 1 \\
\hline Ukraine & 2006 & 2016 & 11 & 1996 & 2011 & 5 \\
\hline United Kingdom & 2006 & 2016 & 11 & 1981 & 2009 & 5 \\
\hline Uruguay & 2006 & 2016 & 11 & 1996 & 2011 & 3 \\
\hline Uzbekistan & 2006 & 2016 & 8 & 2011 & 2011 & 1 \\
\hline Venezuela, RB & 2006 & 2016 & 11 & 1996 & 2000 & 2 \\
\hline Zambia & 2006 & 2016 & 10 & 2007 & 2007 & 1 \\
\hline Zimbabwe & 2006 & 2016 & 11 & 2001 & 2012 & 2 \\
\hline State of Palestine & 2006 & 2016 & 11 & & & 0 \\
\hline Somaliland region & 2009 & 2012 & 4 & & & 0 \\
\hline Nagorno-Karabakh Region & 2013 & 2013 & 1 & & & 0 \\
\hline
\end{tabular}




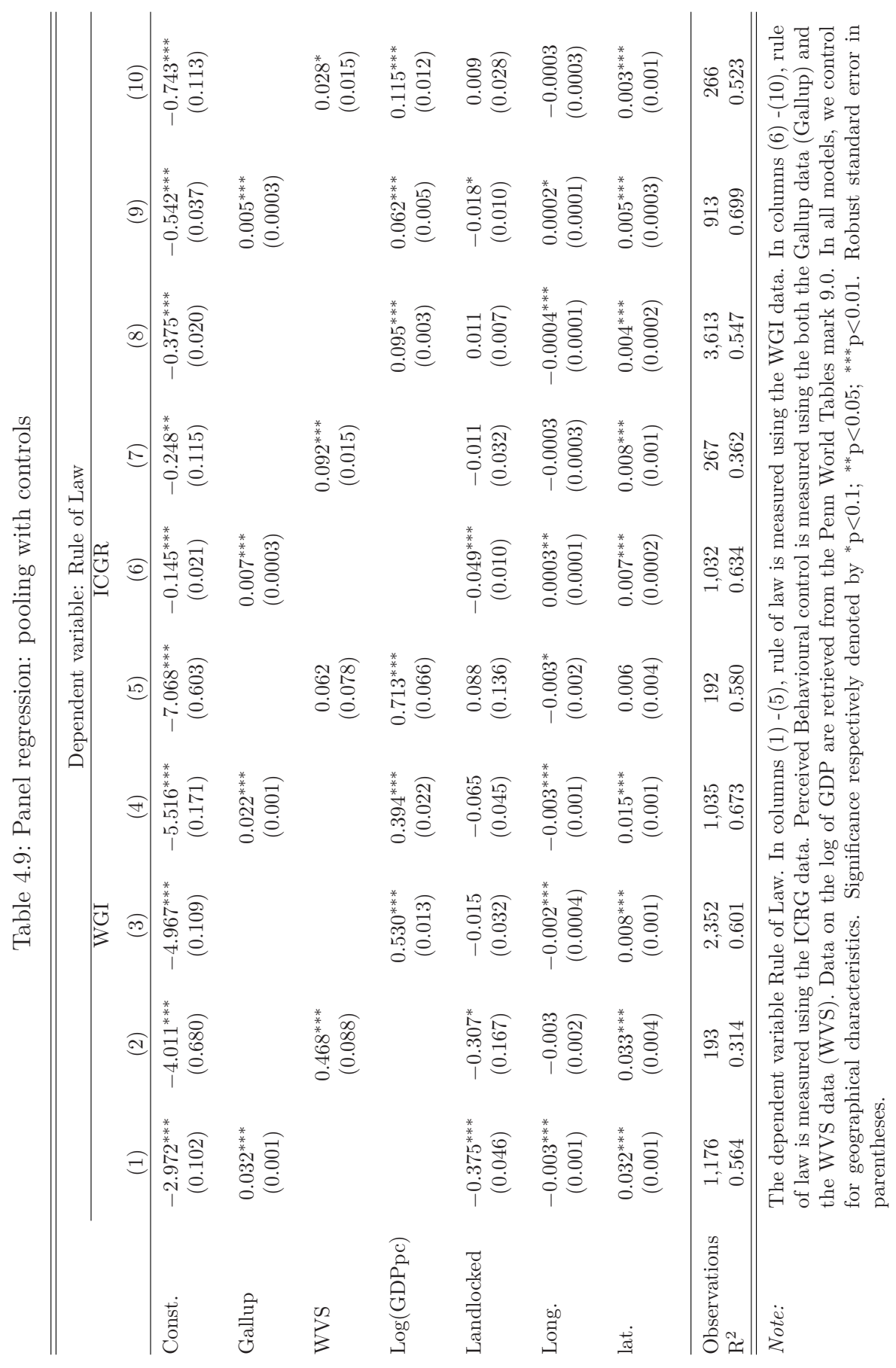




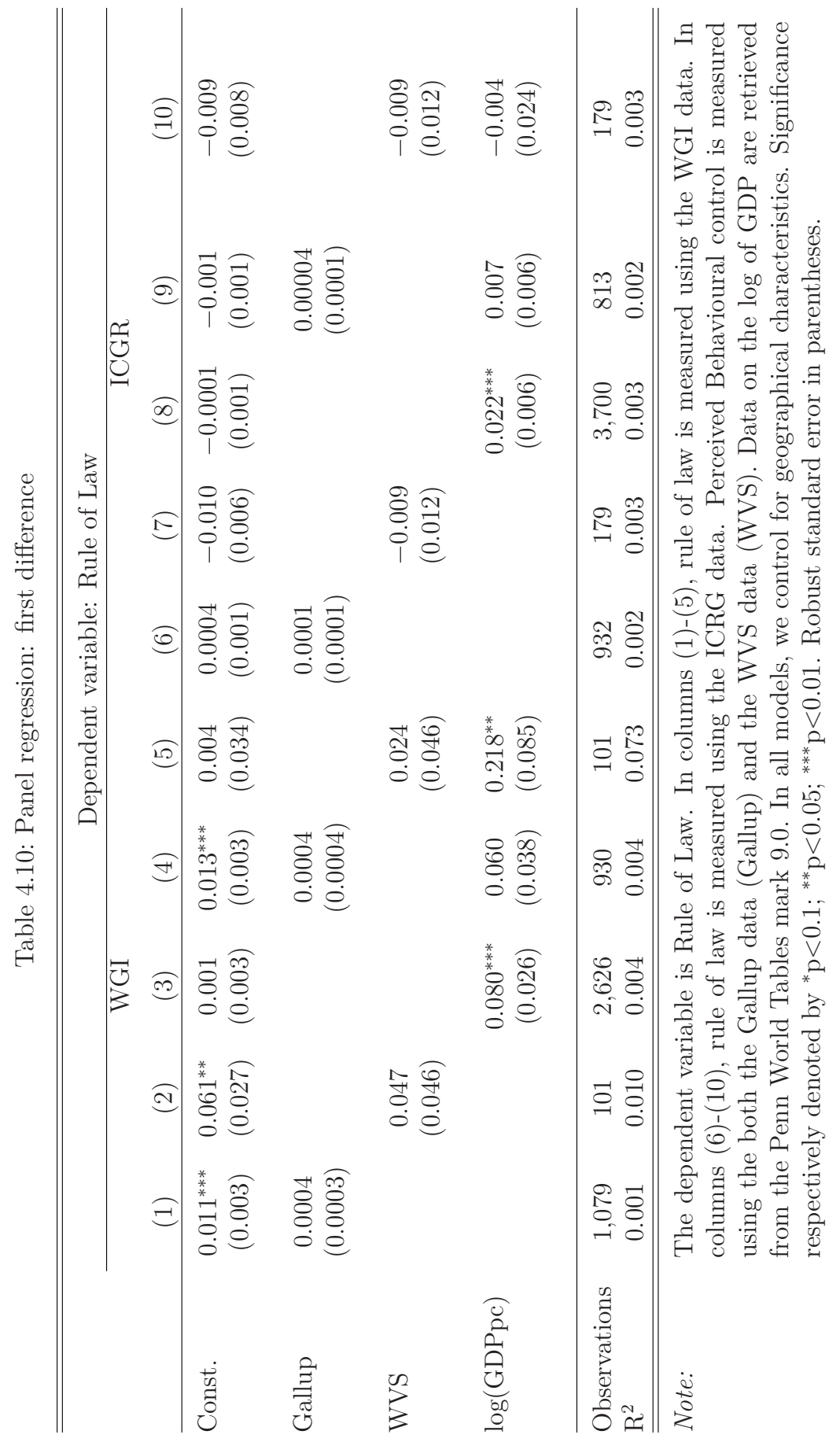


Table 4.11: Panel regression: pooling and individual fixed effects

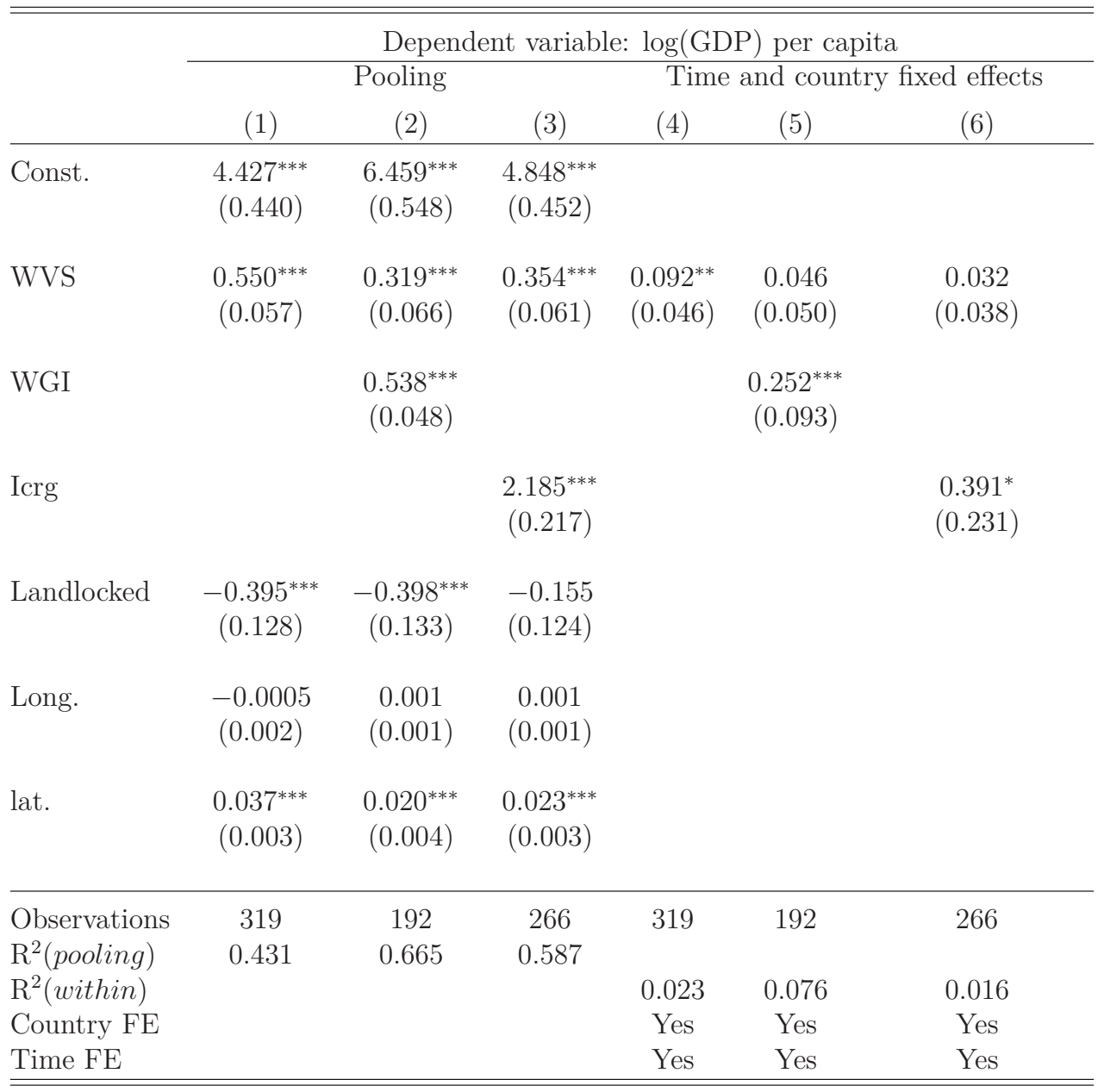

Note: $\quad$ Log GDP (at purchasing power parity) per capita from the Penn World Tables. In columns (1) -(3), we run a pooling model. In columns (4) -(6), I consider country and time fixed effects. Perceived Behavioural control is measured using the WVS data. Institutions are measured using both the WGI and the ICRG data alternatively. In columns (1)(3), we control for geographical characteristics. Significance respectively denoted by ${ }^{*} \mathrm{p}<0.1 ;{ }^{* *} \mathrm{p}<0.05 ;{ }^{* *} \mathrm{p}<0.01$. Robust standard error in parentheses. 
Table 4.12: First stage results of IV regression: using Gallup as a measure for Mental Models, and Icrg for insttitutions

\begin{tabular}{|c|c|c|c|c|}
\hline & MM only i & strumented & MM and Inst & instrumented \\
\hline & Dep. var.: $M M$ & Dep. var.: $M M$ & Dep. var.: $M M$ & Dep. var.: Inst. \\
\hline & $(1)$ & $(2)$ & $(3)$ & $(4)$ \\
\hline Const. & $\begin{array}{c}0.177 \\
(0.110)\end{array}$ & $\begin{array}{c}0.191^{*} \\
(0.113)\end{array}$ & $\begin{array}{c}0.160 \\
(0.143)\end{array}$ & $\begin{array}{r}-0.040 \\
(0.126)\end{array}$ \\
\hline Dist. Norway & $\begin{array}{c}-0.554^{* * *} \\
(0.113)\end{array}$ & $\begin{array}{c}-0.609^{* * *} \\
(0.101)\end{array}$ & $\begin{array}{c}-0.636^{* * *} \\
(0.120)\end{array}$ & $\begin{array}{r}-0.099 \\
(0.124)\end{array}$ \\
\hline Icrg & & $\begin{array}{l}0.393^{* * *} \\
(0.122)\end{array}$ & & \\
\hline Sett. & & & $\begin{array}{r}-0.072 \\
(0.113)\end{array}$ & $\begin{array}{r}-0.111 \\
(0.128)\end{array}$ \\
\hline Lat. & & $\begin{array}{r}-0.247^{*} \\
(0.144)\end{array}$ & $\begin{array}{r}-0.125 \\
(0.153)\end{array}$ & $\begin{array}{c}0.338^{* *} \\
(0.142)\end{array}$ \\
\hline Long. & & $\begin{array}{c}0.160 \\
(0.101)\end{array}$ & $\begin{array}{c}0.172^{*} \\
(0.101)\end{array}$ & $\begin{array}{c}0.045 \\
(0.109)\end{array}$ \\
\hline Landlocked & & $\begin{array}{r}-0.043 \\
(0.282)\end{array}$ & $\begin{array}{r}-0.059 \\
(0.273)\end{array}$ & $\begin{array}{r}-0.066 \\
(0.184) \\
\end{array}$ \\
\hline F-Stat. (Gallup) & 23.92 & 36.03 & 16.79 & \\
\hline F-Stat. (Icrg) & & & & 1.68 \\
\hline Observations & 54 & 54 & 54 & 54 \\
\hline $\mathrm{R}^{2}$ & 0.293 & 0.433 & 0.340 & 0.201 \\
\hline Residual Std. Error & 0.711 & 0.663 & 0.715 & 0.695 \\
\hline
\end{tabular}

Note:

Columns (1-2) dependent variable is Mental models measure using the Gallup data. In these two columns, only mental model is instrumented using the genetic distance from Norway. Lat, Long and landlocked represent the usual geographical variables. Institutions are instead measured using Icrg data. In columns (3-4) both Mental models and Institutions are instrumented using respectively the genetic distance from Norway (Dist. Norway) and Settler mortality data (Sett). Specifically column 3 presents the first stage for mental models and column (4) the one for institutions. Note that the"rule of thumb" according to which the first stage F-statistics should be above 10 as suggested by Staiger et al. [1997] is strictly applicable to the case in which there is a unique endogenous regressor. Thus, this does not apply in column (3-4) when both Institutions and Mental models are instrumented. Significance respectively denoted by ${ }^{*} \mathrm{p}<0.1 ;{ }^{* *} \mathrm{p}<0.05 ;{ }^{* *} \mathrm{p}<0.01$. Robust standard error in parentheses. 
Table 4.13: First stage results of IV regression: using Gallup as a measure for Mental Models, and WGI for institutions

\begin{tabular}{lcccc}
\hline \hline & \multicolumn{2}{c}{ MM only instrumented } & \multicolumn{2}{c}{ MM and Inst. instrumented } \\
& $(1)$ & $(2)$ & $(3)$ & $(4)$ \\
\cline { 2 - 5 } & $0.219^{* *}$ & 0.144 & 0.215 & 0.072 \\
Const. & $(0.110)$ & $(0.113)$ & $(0.143)$ & $(0.126)$ \\
Dist. Norway & $-0.532^{* * *}$ & $-0.379^{* * *}$ & $-0.604^{* * *}$ & $-0.349^{* * *}$ \\
WGI & $(0.113)$ & $(0.101)$ & $(0.120)$ & $(0.124)$ \\
Sett. & & 0.583 & & \\
Lat. & & & & \\
& & & -0.072 & $-0.215^{*}$ \\
Long. & & $-0.332^{* *}$ & -0.096 & $(0.128)$ \\
& & $(0.144)$ & $(0.153)$ & $0.366^{* * *}$ \\
Landlocked & & 0.109 & 0.161 & $0.142)$ \\
& & $(0.101)$ & $(0.101)$ & $(0.109)$ \\
\hline F-Stat. (Gallup) & & -0.166 & -0.021 & 0.278 \\
F-Stat. (WGI) & 27.2 & $(0.282)$ & $(0.273)$ & $(0.184)$ \\
Observations & & 14.78 & 18.28 & \\
R & & & & 7.89 \\
Residual Std. Error & 56 & 56 & 56 & 56 \\
\hline \hline
\end{tabular}

Note:

Columns (1-2) dependent variable is Mental models measure using the Gallup data. In these two columns, only mental models are instrumented using the genetic distance from Norway. Lat, Long and landlocked represent the usual geographical variables. Institutions are instead measured using WGI data. In columns (3-4) both Mental models and Institutions are instrumented using respectively the genetic distance from Norway (Dist. Norway) and Settler mortality data (Sett). Specifically column 3 presents the first stage for mental models and column (4) the one for institutions. Note that the"rule of thumb" according to which the first stage F-statistics should be above 10 as suggested by Staiger et al. [1997] is strictly applicable to the case in which there is a unique endogenous regressor. Thus, this does not apply in column (3-4) when both Institutions and Mental models are instrumented. Significance respectively denoted by ${ }^{*} \mathrm{p}<0.1 ;{ }^{* *} \mathrm{p}<0.05 ;{ }^{* * *} \mathrm{p}<0.01$. Robust standard error in parentheses. 
Table 4.14: Relative effects of institutions (Icrg) and mental models (WVS): on economic development

\begin{tabular}{|c|c|c|c|c|c|c|}
\hline & \multicolumn{6}{|c|}{ Dependent variable: log income 2010} \\
\hline & \multicolumn{3}{|c|}{ OLS } & \multicolumn{2}{|c|}{ IV+controlling inst } & \multirow{2}{*}{$\begin{array}{l}\text { IV } \\
(6) \\
\end{array}$} \\
\hline & $(1)$ & $(2)$ & $(3)$ & $(4)$ & $(5)$ & \\
\hline Const. & $\begin{array}{c}0.000 \\
(0.111)\end{array}$ & $\begin{array}{c}0.000 \\
(0.070)\end{array}$ & $\begin{array}{c}0.049 \\
(0.060)\end{array}$ & $\begin{array}{c}-0.842^{* * *} \\
(0.308)\end{array}$ & $\begin{array}{l}-0.128 \\
(0.154)\end{array}$ & $\begin{array}{l}-0.107 \\
(0.145)\end{array}$ \\
\hline WVS & $\begin{array}{c}0.273^{* *} \\
(0.109)\end{array}$ & & $\begin{array}{l}0.171^{* *} \\
(0.071)\end{array}$ & $\begin{array}{l}1.625^{* *} \\
(0.673)\end{array}$ & $\begin{array}{c}0.739^{* *} \\
(0.322)\end{array}$ & $\begin{array}{c}0.665^{* *} \\
(0.298)\end{array}$ \\
\hline Icrg & & $\begin{array}{c}0.795^{* * *} \\
(0.077)\end{array}$ & $\begin{array}{c}0.532^{* * *} \\
(0.082)\end{array}$ & & $\begin{array}{c}0.450^{* *} \\
(0.220)\end{array}$ & $\begin{array}{c}0.678^{* * *} \\
(0.219)\end{array}$ \\
\hline Lat. & & & $\begin{array}{c}0.394^{* * *} \\
(0.079)\end{array}$ & & $\begin{array}{c}0.492^{* * *} \\
(0.188)\end{array}$ & $\begin{array}{c}0.380^{* *} \\
(0.186)\end{array}$ \\
\hline Long. & & & $\begin{array}{l}-0.073 \\
(0.060)\end{array}$ & & $\begin{array}{l}-0.093 \\
(0.080)\end{array}$ & $\begin{array}{c}-0.117^{*} \\
(0.067)\end{array}$ \\
\hline Landlocked & & & $\begin{array}{c}-0.360^{* *} \\
(0.178)\end{array}$ & & $\begin{array}{l}-0.443 \\
(0.357)\end{array}$ & $\begin{array}{l}-0.429 \\
(0.363)\end{array}$ \\
\hline 1st stage F-Stat. (WVS) & & & & 4.84 & 4.9 & 3.88 \\
\hline 1st stage F-Stat. (Icrg) & & & & & & 7.91 \\
\hline Observations & 74 & 74 & 74 & 33 & 33 & 33 \\
\hline $\mathrm{R}^{2}$ & 0.075 & 0.632 & 0.765 & -0.984 & 0.703 & 0.734 \\
\hline Residual Std. Error & 0.969 & 0.611 & 0.503 & 1.391 & 0.577 & 0.546 \\
\hline
\end{tabular}

Note:

The dependent variable in the second stage is log income (at purchasing power parity) per capita in 2010 from the Penn World Tables. Perceived Behavioural control is measured using the WVS data. Institutions are measure by the ICRG data. In columns (4) and (6), Perceived Behavioural control is instrumented using the geometric distance of frequency of blood types $\mathrm{A}$ and $\mathrm{B}$ in a given country relative to the frequency of blood types A and B in Norway. In column (6), institutions are instrumented using settler mortality data. Latitude, longitude and a dummy variable for landlocked countries is used as controls in columns (3), (5), (6). All coefficients are standardised. Significance respectively denoted by ${ }^{*} \mathrm{p}<0.1 ;{ }^{* *} \mathrm{p}<0.05 ;{ }^{* * *} \mathrm{p}<0.01$. Robust standard error in parentheses. 
Table 4.15: First stage results of IV regression: using WVS as a measure for Mental Models, and Icrg for institutions

\begin{tabular}{|c|c|c|c|c|}
\hline & MM only ir & strumented & MM and Inst. & instrumented \\
\hline & Dep. var.: $M M$ & Dep. var.: $M M$ & Dep. var.: $M M$ & Dep. var.: Inst. \\
\hline & $(1)$ & $(2)$ & $(3)$ & $(4)$ \\
\hline Const. & $\begin{array}{c}0.369^{* *} \\
(0.147)\end{array}$ & $\begin{array}{c}0.140 \\
(0.195)\end{array}$ & $\begin{array}{c}0.042 \\
(0.222)\end{array}$ & $\begin{array}{c}-0.265^{* *} \\
(0.124)\end{array}$ \\
\hline Dist & $\begin{array}{c}-0.406^{* *} \\
(0.185)\end{array}$ & $\begin{array}{c}-0.457^{* *} \\
(0.206)\end{array}$ & $\begin{array}{c}-0.529^{* *} \\
(0.243)\end{array}$ & $\begin{array}{r}-0.062 \\
(0.126)\end{array}$ \\
\hline Icrg & & $\begin{array}{l}0.593^{* * *} \\
(0.229)\end{array}$ & & \\
\hline Sett. & & & $\begin{array}{r}-0.118 \\
(0.148)\end{array}$ & $\begin{array}{c}-0.390^{* * *} \\
(0.099)\end{array}$ \\
\hline Lat. & & $\begin{array}{c}-0.789^{* * *} \\
(0.247)\end{array}$ & $\begin{array}{c}-0.665^{* *} \\
(0.290)\end{array}$ & $\begin{array}{c}0.132 \\
(0.143)\end{array}$ \\
\hline Long. & & $\begin{array}{r}-0.023 \\
(0.150)\end{array}$ & $\begin{array}{c}0.032 \\
(0.157)\end{array}$ & $\begin{array}{c}0.063 \\
(0.114)\end{array}$ \\
\hline Landlocked & & $\begin{array}{r}-0.718 \\
(0.454) \\
\end{array}$ & $\begin{array}{c}-0.844^{* * *} \\
(0.323)\end{array}$ & $\begin{array}{r}-0.065 \\
(0.311) \\
\end{array}$ \\
\hline F-Stat. (WVS) & 4.84 & 4.9 & 3.88 & \\
\hline F-Stat. (Icrg) & & & & 7.91 \\
\hline Observations & 33 & 33 & 33 & 33 \\
\hline $\mathrm{R}^{2}$ & 0.112 & 0.409 & 0.305 & 0.481 \\
\hline Residual Std. Error & 0.996 & 0.871 & 0.945 & 0.540 \\
\hline
\end{tabular}

Note:

Columns (1-2) dependent variable is Mental models which are measured using the WVS data. In these two columns, only mental models are instrumented using the genetic distance from Norway. Lat, Long and landlocked represent the usual geographical variables. Institutions are instead measured using Icrg data. In columns (3-4) both Mental models and Institutions are instrumented using respectively the genetic distance from Norway (Dist. Norway) and Settler mortality data (Sett). Specifically column 3 presents the first stage for mental models and column (4) the one for institutions. Note that the"rule of thumb" according to which the first stage F-statistics should be above 10 as suggested by Staiger et al. [1997] is strictly applicable to the case in which there is a unique endogenous regressor. Thus, this does not apply in column (3-4) when both Institutions and Mental models are instrumented. Significance respectively denoted by ${ }^{*} \mathrm{p}<0.1 ;{ }^{* *} \mathrm{p}<0.05 ;{ }^{* * *} \mathrm{p}<0.01$. Robust standard error in parentheses. 
Table 4.16: Relative effects of institutions (WGI) and mental models (WVS) on economic development

\begin{tabular}{|c|c|c|c|c|c|c|}
\hline & \multicolumn{6}{|c|}{ Dependent variable: log income 2010} \\
\hline & \multicolumn{3}{|c|}{ OLS } & \multicolumn{2}{|c|}{ IV+controlling inst } & \multirow{2}{*}{$\begin{array}{l}\text { IV } \\
(6) \\
\end{array}$} \\
\hline & $(1)$ & $(2)$ & $(3)$ & $(4)$ & $(5)$ & \\
\hline Const. & $\begin{array}{c}-0.000 \\
(0.110)\end{array}$ & $\begin{array}{l}-0.000 \\
(0.073)\end{array}$ & $\begin{array}{c}0.086 \\
(0.064)\end{array}$ & $\begin{array}{c}-0.790^{* * *} \\
(0.285)\end{array}$ & $\begin{array}{l}-0.119 \\
(0.128)\end{array}$ & $\begin{array}{c}-0.074 \\
(0.129)\end{array}$ \\
\hline WVS & $\begin{array}{c}0.269^{* *} \\
(0.106)\end{array}$ & & $\begin{array}{c}0.238^{* * *} \\
(0.067)\end{array}$ & $\begin{array}{l}1.506^{* *} \\
(0.598)\end{array}$ & $\begin{array}{c}0.634^{* *} \\
(0.291)\end{array}$ & $\begin{array}{c}0.354 \\
(0.329)\end{array}$ \\
\hline WGI & & $\begin{array}{c}0.765^{* * *} \\
(0.069)\end{array}$ & $\begin{array}{c}0.473^{* * *} \\
(0.074)\end{array}$ & & $\begin{array}{c}0.348^{* *} \\
(0.138)\end{array}$ & $\begin{array}{c}0.746^{* *} \\
(0.332)\end{array}$ \\
\hline Lat. & & & $\begin{array}{c}0.381^{* * *} \\
(0.092)\end{array}$ & & $\begin{array}{c}0.441^{* *} \\
(0.202)\end{array}$ & $\begin{array}{c}0.132 \\
(0.281)\end{array}$ \\
\hline Long. & & & $\begin{array}{c}0.044 \\
(0.057)\end{array}$ & & $\begin{array}{l}-0.053 \\
(0.085)\end{array}$ & $\begin{array}{l}-0.062 \\
(0.093)\end{array}$ \\
\hline Landlocked & & & $\begin{array}{c}-0.597^{* * *} \\
(0.166)\end{array}$ & & $\begin{array}{c}-0.736^{*} \\
(0.428)\end{array}$ & $\begin{array}{r}-1.062^{* *} \\
(0.435)\end{array}$ \\
\hline 1st stage F-Stat. (WVS) & & & & 5.3 & 4.6 & 4.01 \\
\hline 1st stage F-Stat. (WGI) & & & & & & 6.8 \\
\hline Observations & 76 & 76 & 76 & 34 & 34 & 34 \\
\hline $\mathrm{R}^{2}$ & 0.072 & 0.585 & 0.735 & -0.818 & 0.727 & 0.706 \\
\hline Residual Std. Error & 0.970 & 0.649 & 0.533 & 1.286 & 0.532 & 0.553 \\
\hline
\end{tabular}

Note:

The dependent variable in the second stage is log income (at purchasing power parity) per capita in 2010 from the Penn World Tables. Perceived Behavioural control is measured using the WVS data. Institutions are measure by the WGI data. In columns (4) and (6), Perceived Behavioural control is instrumented using the geometric distance of frequency of blood types A and B in a given country relative to the frequency of blood types A and B in Norway. In column (6), institutions are instrumented using settler mortality data. Latitude, longitude and a dummy variable for landlocked countries is used as controls in columns (3), (5), (6). All coefficients are standardised. Significance respectively denoted by ${ }^{*} \mathrm{p}<0.1 ;{ }^{* *} \mathrm{p}<0.05 ;{ }^{* * *} \mathrm{p}<0.01$. Robust standard error in parentheses. 
Table 4.17: First stage results of IV regression: using WVS as a measure for Mental Models, and WGI for institutions

\begin{tabular}{|c|c|c|c|c|}
\hline & MM only ir & strumented & MM and Inst. & instrumented \\
\hline & Dep. var.: $M M$ & Dep. var.: $M M$ & Dep. var.: $M M$ & Dep. var.: Inst. \\
\hline & $(1)$ & $(2)$ & $(3)$ & $(4)$ \\
\hline Const. & $\begin{array}{c}0.375^{* * *} \\
(0.142)\end{array}$ & $\begin{array}{c}0.122 \\
(0.190)\end{array}$ & $\begin{array}{c}0.050 \\
(0.218)\end{array}$ & $\begin{array}{r}-0.232 \\
(0.179)\end{array}$ \\
\hline Dist & $\begin{array}{c}-0.418^{* *} \\
(0.181)\end{array}$ & $\begin{array}{c}-0.466^{* *} \\
(0.217)\end{array}$ & $\begin{array}{c}-0.508^{* *} \\
(0.229)\end{array}$ & $\begin{array}{c}-0.273^{* *} \\
(0.138)\end{array}$ \\
\hline WGI & & $\begin{array}{c}0.203 \\
(0.172)\end{array}$ & & \\
\hline Sett. & & & $\begin{array}{r}-0.126 \\
(0.147)\end{array}$ & $\begin{array}{c}-0.387^{* * *} \\
(0.111)\end{array}$ \\
\hline Lat. & & $\begin{array}{c}-0.675^{* *} \\
(0.281)\end{array}$ & $\begin{array}{c}-0.662^{* *} \\
(0.280)\end{array}$ & $\begin{array}{c}0.173 \\
(0.174)\end{array}$ \\
\hline Long. & & $\begin{array}{c}0.039 \\
(0.150)\end{array}$ & $\begin{array}{c}0.031 \\
(0.157)\end{array}$ & $\begin{array}{r}-0.001 \\
(0.114)\end{array}$ \\
\hline Landlocked & & $\begin{array}{c}-0.963^{* * *} \\
(0.355)\end{array}$ & $\begin{array}{c}-0.833^{* *} \\
(0.326)\end{array}$ & $\begin{array}{r}0.460^{*} \\
(0.237) \\
\end{array}$ \\
\hline F-Stat. (WVS) & 5.3 & 4.6 & 4.01 & \\
\hline F-Stat. (WGI) & & & & 6.8 \\
\hline Observations & 34 & 34 & 34 & 34 \\
\hline $\mathrm{R}^{2}$ & 0.121 & 0.319 & 0.310 & 0.441 \\
\hline Residual Std. Error & 0.989 & 0.931 & 0.937 & 0.673 \\
\hline
\end{tabular}

Note:

Columns (1-2) dependent variable is Mental models which are measured using the WVS data. In these two columns, only mental models are instrumented using the genetic distance from Norway. Lat., Long and landlocked represent the usual geographical variables. Institutions are instead measured using WGI data. In columns (3-4) both Mental models and Institutions are instrumented using respectively the genetic distance from Norway (Dist. Norway) and Settler mortality data (Sett). Specifically column 3 presents the first stage for mental models and column (4) the one for institutions. Note that the"rule of thumb" according to which the first stage F-statistics should be above 10 as suggested by Staiger et al. [1997] is strictly applicable to the case in which there is a unique endogenous regressor. Thus, this does not apply in column (3-4) when both Institutions and Mental models are instrumented. Significance respectively denoted by ${ }^{*} \mathrm{p}<0.1 ;{ }^{* *} \mathrm{p}<0.05 ;{ }^{* * *} \mathrm{p}<0.01$. Robust standard error in parentheses. 
Table 4.18: Relative effects of institutions (Icrg) and mental models (Gallup) on economic development-Instrument Norway

\begin{tabular}{|c|c|c|c|c|c|c|}
\hline & \multicolumn{6}{|c|}{ Dependent variable: log income per worker 2000} \\
\hline & \multicolumn{3}{|c|}{ OLS } & \multicolumn{2}{|c|}{ IV + controlling inst } & \multirow{2}{*}{$\begin{array}{l}\text { IV } \\
(6)\end{array}$} \\
\hline & $(1)$ & $(2)$ & $(3)$ & $(4)$ & $(5)$ & \\
\hline Const. & $\begin{array}{c}9.475^{* * *} \\
(0.086)\end{array}$ & $\begin{array}{c}9.492^{* * *} \\
(0.061)\end{array}$ & $\begin{array}{c}9.544^{* * *} \\
(0.057)\end{array}$ & $\begin{array}{c}9.141^{* * *} \\
(0.131)\end{array}$ & $\begin{array}{c}9.457^{* * *} \\
(0.121)\end{array}$ & $\begin{array}{c}9.499^{* * *} \\
(0.222)\end{array}$ \\
\hline Gallup & $\begin{array}{c}0.588^{* * *} \\
(0.072)\end{array}$ & & $\begin{array}{c}0.151^{* *} \\
(0.071)\end{array}$ & $\begin{array}{c}1.280^{* * *} \\
(0.245)\end{array}$ & $\begin{array}{c}0.862^{* * *} \\
(0.223)\end{array}$ & $\begin{array}{c}0.467 \\
(0.606)\end{array}$ \\
\hline Icrg & & $\begin{array}{c}0.884^{* * *} \\
(0.071)\end{array}$ & $\begin{array}{c}0.640^{* * *} \\
(0.085)\end{array}$ & & $\begin{array}{c}0.434^{* *} \\
(0.187)\end{array}$ & $\begin{array}{c}2.234 \\
(2.083)\end{array}$ \\
\hline Lat. & & & $\begin{array}{c}0.317^{* * *} \\
(0.056)\end{array}$ & & $\begin{array}{c}0.234 \\
(0.155)\end{array}$ & $\begin{array}{l}-0.487 \\
(0.881)\end{array}$ \\
\hline Long. & & & $\begin{array}{c}-0.089^{*} \\
(0.046)\end{array}$ & & $\begin{array}{c}-0.065 \\
(0.081)\end{array}$ & $\begin{array}{c}-0.116 \\
(0.169)\end{array}$ \\
\hline Landlocked & & & $\begin{array}{c}-0.321^{* *} \\
(0.159)\end{array}$ & & $\begin{array}{c}-0.379 \\
(0.379)\end{array}$ & $\begin{array}{c}-0.224 \\
(0.427)\end{array}$ \\
\hline $\begin{array}{l}\text { 1st stage F-Stat. (Gallup) } \\
\text { 1st stage F-Stat. (Icrg) }\end{array}$ & & & & 23.92 & 36.03 & 16.79 \\
\hline $\begin{array}{l}\text { 1st stage F-Stat. (1crg) } \\
\text { Observations }\end{array}$ & 119 & 119 & 119 & 54 & 54 & $\begin{array}{c}1.68 \\
54\end{array}$ \\
\hline $\mathrm{R}^{2}$ & 0.283 & 0.628 & 0.717 & 0.086 & 0.492 & -0.519 \\
\hline Residual Std. Error & 0.942 & 0.678 & 0.603 & 0.982 & 0.762 & 1.318 \\
\hline
\end{tabular}

\section{Note:}

The dependent variable in the second stage is log income (at purchasing power parity) per worker in 2000 from the Penn World Tables. Perceived Behavioural control is measured using the Gallup data. Institutions are measure by the ICRG data. In columns (4) and (6), Perceived Behavioural control is instrumented using the geometric distance of frequency of blood types A and B in a given country relative to the frequency of blood types A and B in Norway. In column (6), institutions are instrumented using settler mortality data. Latitude, longitude and a dummy variable for landlocked countries are used as controls in columns (3), (5), (6). All coefficients are standardised. Significance respectively denoted by ${ }^{*} \mathrm{p}<0.1 ;{ }^{* *} \mathrm{p}<0.05 ;{ }^{* *} \mathrm{p}<0.01$. Robust standard error in parentheses. 
Table 4.19: Relative effects of institutions (WGI) and mental models (Gallup) on economic development-Instrument Norway

\begin{tabular}{|c|c|c|c|c|c|c|}
\hline & \multicolumn{6}{|c|}{ Dependent variable: log income per worker 2000} \\
\hline & \multicolumn{3}{|c|}{ OLS } & \multicolumn{2}{|c|}{ IV + controlling inst } & \multirow{2}{*}{$\begin{array}{l}\text { IV } \\
(6)\end{array}$} \\
\hline & $(1)$ & $(2)$ & $(3)$ & $(4)$ & $(5)$ & \\
\hline Const. & $\begin{array}{c}9.308^{* * *} \\
(0.084)\end{array}$ & $\begin{array}{c}9.311^{* * *} \\
(0.063)\end{array}$ & $\begin{array}{c}9.408^{* * *} \\
(0.063)\end{array}$ & $\begin{array}{c}8.964^{* * *} \\
(0.127)\end{array}$ & $\begin{array}{c}9.324^{* * *} \\
(0.123)\end{array}$ & $\begin{array}{r}9.308^{* * *} \\
(0.193)\end{array}$ \\
\hline Gallup & $\begin{array}{c}0.585^{* * *} \\
(0.075)\end{array}$ & & $\begin{array}{l}0.131^{*} \\
(0.078)\end{array}$ & $\begin{array}{c}1.370^{* * *} \\
(0.248)\end{array}$ & $\begin{array}{l}1.047^{* *} \\
(0.447)\end{array}$ & $\begin{array}{c}0.116 \\
(0.510)\end{array}$ \\
\hline WGI & & $\begin{array}{c}0.883^{* * *} \\
(0.050)\end{array}$ & $\begin{array}{c}0.587^{* * *} \\
(0.097)\end{array}$ & & $\begin{array}{l}-0.018 \\
(0.383)\end{array}$ & $\begin{array}{l}1.335^{* *} \\
(0.652)\end{array}$ \\
\hline Lat. & & & $\begin{array}{c}0.322^{* * *} \\
(0.077)\end{array}$ & & $\begin{array}{l}0.445^{*} \\
(0.239)\end{array}$ & $\begin{array}{l}-0.231 \\
(0.385)\end{array}$ \\
\hline Long. & & & $\begin{array}{l}-0.067 \\
(0.062)\end{array}$ & & $\begin{array}{l}-0.052 \\
(0.104)\end{array}$ & $\begin{array}{l}-0.045 \\
(0.109)\end{array}$ \\
\hline Landlocked & & & $\begin{aligned}-0.402^{* * *} \\
(0.155)\end{aligned}$ & & $\begin{array}{l}-0.378 \\
(0.466)\end{array}$ & $\begin{array}{l}-0.702 \\
(0.430)\end{array}$ \\
\hline $\begin{array}{l}\text { 1st stage F-Stat. (Gallup) } \\
\text { 1st stage F-Stat. (WGI) }\end{array}$ & & & & 27.2 & 14.78 & $\begin{array}{c}18.28 \\
7.89\end{array}$ \\
\hline Observations & 141 & 141 & 141 & 56 & 56 & 56 \\
\hline $\mathrm{R}^{2}$ & 0.255 & 0.579 & 0.642 & 0.169 & 0.411 & 0.302 \\
\hline Residual Std. Error & 1.006 & 0.756 & 0.708 & 0.963 & 0.842 & 0.917 \\
\hline
\end{tabular}

Note:

The dependent variable in the second stage is log income (at purchasing power parity) per worker in 2000 from the Penn World Tables. Perceived Behavioural control is measured using the Gallup data. Institutions are measured by the WGI data. In columns (4) and (6), Perceived Behavioural control is instrumented using the geometric distance of frequency of blood types A and B in a given country relative to the frequency of blood types A and B in Norway. In column (6), institutions are instrumented using settler mortality data. Latitude, longitude and a dummy variable for landlocked countries are used as controls in columns (3), (5), (6). All coefficients are standardised. Significance respectively denoted by ${ }^{*} \mathrm{p}<0.1 ;{ }^{* *} \mathrm{p}<0.05 ;{ }^{* *} \mathrm{p}<0.01$. Robust standard error in parentheses. 
Table 4.20: Relative effects of institutions (Icrg) and mental models (WVS) on economic development-Instrument Norway

\begin{tabular}{|c|c|c|c|c|c|c|}
\hline & \multicolumn{6}{|c|}{ Dependent variable: log income per worker 2000} \\
\hline & \multicolumn{3}{|c|}{ OLS } & \multicolumn{2}{|c|}{ IV + controlling inst } & \multirow{2}{*}{$\begin{array}{l}\text { IV } \\
(6) \\
\end{array}$} \\
\hline & $(1)$ & $(2)$ & $(3)$ & $(4)$ & $(5)$ & \\
\hline Const. & $\begin{array}{c}9.791^{* * *} \\
(0.120)\end{array}$ & $\begin{array}{c}9.805^{* * *} \\
(0.075)\end{array}$ & $\begin{array}{c}9.837^{* * *} \\
(0.063)\end{array}$ & $\begin{array}{c}8.966^{* * *} \\
(0.359)\end{array}$ & $\begin{array}{c}9.851^{* * *} \\
(0.163)\end{array}$ & $\begin{array}{c}9.902^{* * *} \\
(0.157)\end{array}$ \\
\hline WVS & $\begin{array}{c}0.284^{* * *} \\
(0.105)\end{array}$ & & $\begin{array}{c}0.162^{* *} \\
(0.070)\end{array}$ & $\begin{array}{l}1.816^{* *} \\
(0.788)\end{array}$ & $\begin{array}{c}0.812^{* *} \\
(0.354)\end{array}$ & $\begin{array}{c}0.637^{*} \\
(0.357)\end{array}$ \\
\hline Icrg & & $\begin{array}{c}0.854^{* * *} \\
(0.094)\end{array}$ & $\begin{array}{c}0.595^{* * *} \\
(0.094)\end{array}$ & & $\begin{array}{c}0.482^{* *} \\
(0.209)\end{array}$ & $\begin{array}{c}1.019^{* * *} \\
(0.305)\end{array}$ \\
\hline Lat. & & & $\begin{array}{c}0.378^{* * *} \\
(0.086)\end{array}$ & & $\begin{array}{c}0.703^{* * *} \\
(0.207)\end{array}$ & $\begin{array}{c}0.439^{*} \\
(0.244)\end{array}$ \\
\hline Long. & & & $\begin{array}{c}-0.119^{*} \\
(0.070)\end{array}$ & & $\begin{array}{c}-0.058 \\
(0.082)\end{array}$ & $\begin{array}{l}-0.115 \\
(0.080)\end{array}$ \\
\hline Landlocked & & & $\begin{array}{c}-0.302 \\
(0.236)\end{array}$ & & $\begin{array}{c}-0.278 \\
(0.403)\end{array}$ & $\begin{array}{l}-0.245 \\
(0.439)\end{array}$ \\
\hline 1st stage F-Stat. (WVS) & & & & 4.84 & 4.9 & 3.88 \\
\hline 1st stage F-Stat. (Icrg) & & & & & & 7.91 \\
\hline Observations & 73 & 73 & 73 & 33 & 33 & 33 \\
\hline $\mathrm{R}^{2}$ & 0.070 & 0.623 & 0.739 & -1.155 & 0.728 & 0.751 \\
\hline Residual Std. Error & 1.041 & 0.663 & 0.567 & 1.601 & 0.610 & 0.583 \\
\hline
\end{tabular}

Note:

The dependent variable in the second stage is log income (at purchasing power parity) per worker in 2000 from the Penn World Tables. Perceived Behavioural control is measured using the WVS data. Institutions are measured by the ICRG data. In columns (4) and (6), Perceived Behavioural control is instrumented using the geometric distance of frequency of blood types A and B in a given country relative to the frequency of blood types A and B in Norway. In column (6), institutions are instrumented using settler mortality data. Latitude, longitude and a dummy variable for landlocked countries are used as controls in columns (3), (5), (6). All coefficients are standardised. Significance respectively denoted by ${ }^{*} \mathrm{p}<0.1 ;{ }^{* *} \mathrm{p}<0.05 ;{ }^{* * *} \mathrm{p}<0.01$. Robust standard error in parentheses. 
Table 4.21: Relative effects of institutions (WGI) and mental models (WVS) on economic development-Instrument Norway

\begin{tabular}{|c|c|c|c|c|c|c|}
\hline & \multicolumn{6}{|c|}{ Dependent variable: log income per worker 2000} \\
\hline & \multicolumn{3}{|c|}{ OLS } & \multicolumn{2}{|c|}{ IV + controlling inst } & \multirow{2}{*}{$\begin{array}{l}\text { IV } \\
(6)\end{array}$} \\
\hline & $(1)$ & $(2)$ & $(3)$ & $(4)$ & $(5)$ & \\
\hline Const. & $\begin{array}{c}9.759^{* * * *} \\
(0.120)\end{array}$ & $\begin{array}{c}9.761^{* * * *} \\
(0.080)\end{array}$ & $\begin{array}{c}9.844^{* * *} \\
(0.071)\end{array}$ & $\begin{array}{c}8.969^{* * *} \\
(0.339)\end{array}$ & $\begin{array}{c}9.809^{* * *} \\
(0.149)\end{array}$ & $\begin{array}{c}9.898^{* * *} \\
(0.183)\end{array}$ \\
\hline WVS & $\begin{array}{c}0.285^{* * *} \\
(0.104)\end{array}$ & & $\begin{array}{c}0.237^{* * *} \\
(0.075)\end{array}$ & $\begin{array}{c}1.719^{* *} \\
(0.716)\end{array}$ & $\begin{array}{c}0.765^{* *} \\
(0.330)\end{array}$ & $\begin{array}{c}0.208 \\
(0.455)\end{array}$ \\
\hline WGI & & $\begin{array}{c}0.834^{* * *} \\
(0.072)\end{array}$ & $\begin{array}{c}0.546^{* * *} \\
(0.088)\end{array}$ & & $\begin{array}{c}0.337^{* *} \\
(0.146)\end{array}$ & $\begin{array}{l}1.130^{* *} \\
(0.457)\end{array}$ \\
\hline Lat. & & & $\begin{array}{c}0.365^{* * *} \\
(0.112)\end{array}$ & & $\begin{array}{c}0.694^{* * *} \\
(0.227)\end{array}$ & $\begin{array}{c}0.079 \\
(0.421)\end{array}$ \\
\hline Long. & & & $\begin{array}{c}0.005 \\
(0.065)\end{array}$ & & $\begin{array}{c}-0.016 \\
(0.090)\end{array}$ & $\begin{array}{l}-0.035 \\
(0.125)\end{array}$ \\
\hline Landlocked & & & $\begin{array}{c}-0.577^{* *} \\
(0.229)\end{array}$ & & $\begin{array}{c}-0.560 \\
(0.505)\end{array}$ & $\begin{array}{c}-1.208^{* *} \\
(0.600)\end{array}$ \\
\hline 1st stage F-Stat. (WVS) & & & & 5.3 & 4.6 & 4.01 \\
\hline 1st stage F-Stat. (WGI) & & & & & & 6.8 \\
\hline Observations & 75 & 75 & 75 & 34 & 34 & 34 \\
\hline $\mathrm{R}^{2}$ & 0.069 & 0.591 & 0.711 & -0.979 & 0.720 & 0.536 \\
\hline Residual Std. Error & 1.058 & 0.701 & 0.607 & 1.512 & 0.608 & 0.783 \\
\hline
\end{tabular}

Note:

The dependent variable in the second stage is log income (at purchasing power parity) per worker in 2000 from the Penn World Tables. Perceived Behavioural control is measured using the WVS data. Institutions are measure by the WGI data. In columns (4) and (6), Perceived Behavioural control is instrumented using the geometric distance of frequency of blood types A and B in a given country relative to the frequency of blood types A and B in Norway. In column (6), institutions are instrumented using settler mortality data. Latitude, longitude and a dummy variable for landlocked countries is used as controls in columns (3), (5), (6). All coefficients are standardised. Significance respectively denoted by ${ }^{*} \mathrm{p}<0.1 ;{ }^{* *} \mathrm{p}<0.05 ;{ }^{* * *} \mathrm{p}<0.01$. Robust standard error in parentheses. 
Table 4.22: Relative effects of institutions (Icrg) and mental models (Gallup) on economic development-Instrument UK

\begin{tabular}{|c|c|c|c|c|c|c|}
\hline & \multicolumn{6}{|c|}{ Dependent variable: log income 2010} \\
\hline & \multicolumn{3}{|c|}{ OLS } & \multicolumn{2}{|c|}{ IV+controlling inst } & \multirow{2}{*}{$\begin{array}{l}\text { IV } \\
(6)\end{array}$} \\
\hline & $(1)$ & $(2)$ & $(3)$ & $(4)$ & $(5)$ & \\
\hline Const. & $\begin{array}{l}-0.000 \\
(0.080)\end{array}$ & $\begin{array}{l}-0.000 \\
(0.057)\end{array}$ & $\begin{array}{c}0.046 \\
(0.054)\end{array}$ & $\begin{array}{c}-0.385^{* * *} \\
(0.111)\end{array}$ & $\begin{array}{l}-0.166 \\
(0.111)\end{array}$ & $\begin{array}{l}-0.134 \\
(0.157)\end{array}$ \\
\hline Gallup & $\begin{array}{c}0.471^{* * *} \\
(0.070)\end{array}$ & & $\begin{array}{c}0.098 \\
(0.062)\end{array}$ & $\begin{array}{c}1.115^{* * *} \\
(0.210)\end{array}$ & $\begin{array}{c}0.846^{* * *} \\
(0.217)\end{array}$ & $\begin{array}{c}0.557 \\
(0.442)\end{array}$ \\
\hline Icrg & & $\begin{array}{c}0.779^{* * *} \\
(0.057)\end{array}$ & $\begin{array}{c}0.544^{* * *} \\
(0.070)\end{array}$ & & $\begin{array}{l}0.286^{*} \\
(0.163)\end{array}$ & $\begin{array}{c}1.445 \\
(1.436)\end{array}$ \\
\hline Lat. & & & $\begin{array}{c}0.375^{* * *} \\
(0.052)\end{array}$ & & $\begin{array}{c}0.127 \\
(0.136)\end{array}$ & $\begin{array}{l}-0.329 \\
(0.601)\end{array}$ \\
\hline Long. & & & $\begin{array}{l}-0.002 \\
(0.042)\end{array}$ & & $\begin{array}{l}-0.015 \\
(0.073)\end{array}$ & $\begin{array}{l}-0.049 \\
(0.105)\end{array}$ \\
\hline Landlocked & & & $\begin{array}{c}-0.249^{*} \\
(0.133)\end{array}$ & & $\begin{array}{l}-0.481 \\
(0.346)\end{array}$ & $\begin{array}{r}-0.394 \\
(0.343)\end{array}$ \\
\hline $\begin{array}{l}\text { 1st stage F-Stat. (Gallup) } \\
\text { 1st stage F-Stat. (Icrg) }\end{array}$ & & & & 21.08 & 29.23 & $\begin{array}{c}14.45 \\
1.95\end{array}$ \\
\hline Observations & 120 & 120 & 120 & 54 & 54 & 54 \\
\hline $\mathrm{R}^{2}$ & 0.222 & 0.607 & 0.723 & 0.122 & 0.435 & -0.038 \\
\hline Residual Std. Error & 0.886 & 0.630 & 0.538 & 0.829 & 0.692 & 0.939 \\
\hline
\end{tabular}

Note:

The dependent variable in the second stage is log income (at purchasing power parity) per capita in 2010 from the Penn World Tables. Perceived Behavioural control is measured using the Gallup data. Institutions are measured by the ICRG data. In columns (4) and (6), Perceived Behavioural control is instrumented using the geometric distance of frequency of blood types A and B in a given country relative to the frequency of blood types A and B in the UK. In column (6), institutions are instrumented using settler mortality data. Latitude, longitude and a dummy variable for landlocked countries are used as controls in columns (3), (5), (6). All coefficients are standardised. Significance respectively denoted by ${ }^{*} \mathrm{p}<0.1 ;{ }^{* *} \mathrm{p}<0.05 ;{ }^{* *} \mathrm{p}<0.01$. Robust standard error in parentheses. 
Table 4.23: Relative effects of institutions (WGI) and mental models (Gallup) on economic development-Instrument UK

\begin{tabular}{|c|c|c|c|c|c|c|}
\hline & \multicolumn{6}{|c|}{ Dependent variable: log income 2010} \\
\hline & \multicolumn{3}{|c|}{ OLS } & \multicolumn{2}{|c|}{ IV+controlling inst } & \multirow{2}{*}{$\begin{array}{l}\text { IV } \\
(6)\end{array}$} \\
\hline & $(1)$ & $(2)$ & $(3)$ & $(4)$ & $(5)$ & \\
\hline Const. & $\begin{array}{c}0.000 \\
(0.074)\end{array}$ & $\begin{array}{c}0.000 \\
(0.054)\end{array}$ & $\begin{array}{c}0.093^{*} \\
(0.054)\end{array}$ & $\begin{array}{c}-0.369^{* * *} \\
(0.104)\end{array}$ & $\begin{array}{l}-0.121 \\
(0.112)\end{array}$ & $\begin{array}{l}-0.131 \\
(0.137)\end{array}$ \\
\hline Gallup & $\begin{array}{c}0.467^{* * *} \\
(0.070)\end{array}$ & & $\begin{array}{c}0.066 \\
(0.067)\end{array}$ & $\begin{array}{c}1.149^{* * *} \\
(0.204)\end{array}$ & $\begin{array}{l}1.015^{* *} \\
(0.400)\end{array}$ & $\begin{array}{c}0.389 \\
(0.429)\end{array}$ \\
\hline WGI & & $\begin{array}{c}0.763^{* * *} \\
(0.045)\end{array}$ & $\begin{array}{c}0.514^{* * *} \\
(0.083)\end{array}$ & & $\begin{array}{c}-0.094 \\
(0.330)\end{array}$ & $\begin{array}{c}0.809 \\
(0.563)\end{array}$ \\
\hline Lat. & & & $\begin{array}{c}0.337^{* * *} \\
(0.061)\end{array}$ & & $\begin{array}{c}0.310 \\
(0.198)\end{array}$ & $\begin{array}{l}-0.141 \\
(0.315)\end{array}$ \\
\hline Long. & & & $\begin{array}{c}0.043 \\
(0.042)\end{array}$ & & $\begin{array}{l}-0.004 \\
(0.086)\end{array}$ & $\begin{array}{c}0.001 \\
(0.080)\end{array}$ \\
\hline Landlocked & & & $\begin{array}{c}-0.381^{* * *} \\
(0.119)\end{array}$ & & $\begin{array}{l}-0.431 \\
(0.397)\end{array}$ & $\begin{array}{r}-0.648^{*} \\
(0.336)\end{array}$ \\
\hline 1st stage F-Stat. (Gallup) & & & & 24.26 & 13.23 & 16.05 \\
\hline 1st stage F-Stat. (WGI) & & & & & & 7.49 \\
\hline Observations & 143 & 143 & 143 & 56 & 56 & 56 \\
\hline $\mathrm{R}^{2}$ & 0.218 & 0.582 & 0.672 & 0.206 & 0.365 & 0.409 \\
\hline Residual Std. Error & 0.887 & 0.649 & 0.583 & 0.785 & 0.729 & 0.703 \\
\hline
\end{tabular}

Note:

The dependent variable in the second stage is log income (at purchasing power parity) per capita in 2010 from the Penn World Tables. Perceived Behavioural control is measured using the Gallup data. Institutions are measured by the WGI data. In columns (4) and (6), Perceived Behavioural control is instrumented using the geometric distance of frequency of blood types $\mathrm{A}$ and $\mathrm{B}$ in a given country relative to the frequency of blood types A and B in the UK. In column (6), institutions are instrumented using settler mortality data. Latitude, longitude and a dummy variable for landlocked countries are used as controls in columns (3), (5), (6). All coefficients are standardised. Significance respectively denoted by ${ }^{*} \mathrm{p}<0.1 ;{ }^{* *} \mathrm{p}<0.05 ;{ }^{* * *} \mathrm{p}<0.01$. Robust standard error in parentheses. 
Table 4.24: Relative effects of institutions (Icrg) and mental models (WVS): on economic development-Instrument UK

\begin{tabular}{|c|c|c|c|c|c|c|}
\hline & \multicolumn{6}{|c|}{ Dependent variable: log income 2010} \\
\hline & \multicolumn{3}{|c|}{ OLS } & \multicolumn{2}{|c|}{ IV+controlling inst } & \multirow{2}{*}{$\begin{array}{l}\text { IV } \\
(6)\end{array}$} \\
\hline & $(1)$ & $(2)$ & $(3)$ & $(4)$ & $(5)$ & \\
\hline Const. & $\begin{array}{c}0.000 \\
(0.111)\end{array}$ & $\begin{array}{c}0.000 \\
(0.070)\end{array}$ & $\begin{array}{c}0.049 \\
(0.060)\end{array}$ & $\begin{array}{c}-0.792^{* * *} \\
(0.250)\end{array}$ & $\begin{array}{c}-0.117 \\
(0.125)\end{array}$ & $\begin{array}{c}-0.098 \\
(0.121)\end{array}$ \\
\hline WVS & $\begin{array}{c}0.273^{* *} \\
(0.109)\end{array}$ & & $\begin{array}{l}0.171^{* *} \\
(0.071)\end{array}$ & $\begin{array}{c}1.349^{* * *} \\
(0.508)\end{array}$ & $\begin{array}{l}0.584^{* *} \\
(0.237)\end{array}$ & $\begin{array}{l}0.523^{* *} \\
(0.218)\end{array}$ \\
\hline Icrg & & $\begin{array}{c}0.795^{* * *} \\
(0.077)\end{array}$ & $\begin{array}{c}0.532^{* * *} \\
(0.082)\end{array}$ & & $\begin{array}{c}0.558^{* * *} \\
(0.189)\end{array}$ & $\begin{array}{c}0.755^{* * *} \\
(0.192)\end{array}$ \\
\hline Lat. & & & $\begin{array}{c}0.394^{* * *} \\
(0.079)\end{array}$ & & $\begin{array}{c}0.393^{* * *} \\
(0.150)\end{array}$ & $\begin{array}{l}0.297^{*} \\
(0.154)\end{array}$ \\
\hline Long. & & & $\begin{array}{c}-0.073 \\
(0.060)\end{array}$ & & $\begin{array}{c}-0.129^{*} \\
(0.070)\end{array}$ & $\begin{array}{c}-0.149^{* *} \\
(0.059)\end{array}$ \\
\hline Landlocked & & & $\begin{array}{c}-0.360^{* *} \\
(0.178)\end{array}$ & & $\begin{array}{c}-0.595^{* *} \\
(0.284)\end{array}$ & $\begin{array}{c}-0.577^{*} \\
(0.310)\end{array}$ \\
\hline 1st stage F-Stat. (WVS) & & & & 6.9 & 7.21 & 5.95 \\
\hline 1st stage F-Stat. (Icrg) & & & & & & 7.73 \\
\hline Observations & 74 & 74 & 74 & 33 & 33 & 33 \\
\hline $\mathrm{R}^{2}$ & 0.075 & 0.632 & 0.765 & -0.390 & 0.783 & 0.795 \\
\hline Residual Std. Error & 0.969 & 0.611 & 0.503 & 1.165 & 0.493 & 0.479 \\
\hline
\end{tabular}

Note:

The dependent variable in the second stage is log income (at purchasing power parity) per capita in 2010 from the Penn World Tables. Perceived Behavioural control is measured using the WVS data. Institutions are measured by the ICRG data. In columns (4) and (6), Perceived Behavioural control is instrumented using the geometric distance of frequency of blood types $\mathrm{A}$ and $\mathrm{B}$ in a given country relative to the frequency of blood types A and B in the UK. In column (6), institutions are instrumented using settler mortality data. Latitude, longitude and a dummy variable for landlocked countries are used as controls in columns (3), (5), (6). All coefficients are standardised. Significance respectively denoted by ${ }^{*} \mathrm{p}<0.1 ;{ }^{* *} \mathrm{p}<0.05 ;{ }^{* * *} \mathrm{p}<0.01$. Robust standard error in parentheses. 
Table 4.25: Relative effects of institutions (WGI) and mental models (WVS) on economic development-Instrument UK

\begin{tabular}{|c|c|c|c|c|c|c|}
\hline & \multicolumn{6}{|c|}{ Dependent variable: log income 2010} \\
\hline & \multicolumn{3}{|c|}{ OLS } & \multicolumn{2}{|c|}{ IV + controlling inst } & \multirow{2}{*}{$\begin{array}{l}\text { IV } \\
(6)\end{array}$} \\
\hline & $(1)$ & $(2)$ & $(3)$ & $(4)$ & $(5)$ & \\
\hline Const. & $\begin{array}{l}-0.000 \\
(0.110)\end{array}$ & $\begin{array}{c}-0.000 \\
(0.073)\end{array}$ & $\begin{array}{c}0.086 \\
(0.064)\end{array}$ & $\begin{array}{c}-0.742^{\text {*** }} \\
(0.234)\end{array}$ & $\begin{array}{l}-0.113 \\
(0.110)\end{array}$ & $\begin{array}{l}-0.070 \\
(0.125)\end{array}$ \\
\hline WVS & $\begin{array}{c}0.269^{* *} \\
(0.106)\end{array}$ & & $\begin{array}{c}0.238^{* * *} \\
(0.067)\end{array}$ & $\begin{array}{c}1.263^{* * *} \\
(0.461)\end{array}$ & $\begin{array}{l}0.523^{* *} \\
(0.229)\end{array}$ & $\begin{array}{c}0.297 \\
(0.254)\end{array}$ \\
\hline WGI & & $\begin{array}{c}0.765^{* * *} \\
(0.069)\end{array}$ & $\begin{array}{c}0.473^{* * *} \\
(0.074)\end{array}$ & & $\begin{array}{c}0.387^{* * *} \\
(0.128)\end{array}$ & $\begin{array}{c}0.775^{* *} \\
(0.316)\end{array}$ \\
\hline Lat. & & & $\begin{array}{c}0.381^{* * *} \\
(0.092)\end{array}$ & & $\begin{array}{c}0.378^{* *} \\
(0.182)\end{array}$ & $\begin{array}{c}0.096 \\
(0.252)\end{array}$ \\
\hline Long. & & & $\begin{array}{c}0.044 \\
(0.057)\end{array}$ & & $\begin{array}{l}-0.071 \\
(0.081)\end{array}$ & $\begin{array}{c}-0.070 \\
(0.093)\end{array}$ \\
\hline Landlocked & & & $\begin{array}{c}-0.597^{* * *} \\
(0.166)\end{array}$ & & $\begin{array}{c}-0.880^{* *} \\
(0.349)\end{array}$ & $\begin{array}{c}-1.135^{\text {*** }} \\
(0.347)\end{array}$ \\
\hline 1st stage F-Stat. (WVS) & & & & 7.4 & 6.72 & 6 \\
\hline 1st stage F-Stat. (WGI) & & & & & & 7.03 \\
\hline Observations & 76 & 76 & 76 & 34 & 34 & 34 \\
\hline $\mathrm{R}^{2}$ & 0.072 & 0.585 & 0.735 & -0.312 & 0.759 & 0.692 \\
\hline Residual Std. Error & 0.970 & 0.649 & 0.533 & 1.092 & 0.501 & 0.566 \\
\hline
\end{tabular}

Note:

The dependent variable in the second stage is log income (at purchasing power parity) per capita in 2010 from the Penn World Tables. Perceived Behavioural control is measured using the WVS data. Institutions are measured by the WGI data. In columns (4) and (6), Perceived Behavioural control is instrumented using the geometric distance of frequency of blood types A and B in a given country relative to the frequency of blood types A and B in the UK. In column (6), institutions are instrumented using settler mortality data. Latitude, longitude and a dummy variable for landlocked countries are used as controls in columns (3), (5), (6). All coefficients are standardised. Significance respectively denoted by ${ }^{*} \mathrm{p}<0.1 ;{ }^{* *} \mathrm{p}<0.05 ;{ }^{* * *} \mathrm{p}<0.01$. Robust standard error in parentheses. 


\section{CHAPTER 5}

\section{Conclusions}

This thesis proposed a micro-founded explanation of institutional inertia.

Institutions can be considered the foundations of social life. They ensure consistency and stability in societies. Nonetheless, some institutions persist over time, in spite of being no longer in line with - or conducive to - economic development. The lack of institutional change is usually explained within the path-dependence framework according to which increasing returns to adoption lock societies into possibly inefficient pathways of development. The path-dependence model relies on an utilitarian mechanism. It assumes that individuals are utility maximisers. The framework gives little consideration their limited cognitive capacities. But, as a matter of fact, social psychologists posit that individuals are boundedly rational and that self-perceptions affect human decisions-making processes and the desirability of rule changes.

As a result, institutional persistence might not be the result of simple rational calculations. Institutions might persist because of some basic human cognitive limitations. In fact, independently from how we define them, institutions are generalised procedures for action which emerge from multiple individual decision-making processes. Thus, human cognition and bounded rationality are likely to affect the evolution of institutions. Therefore, this thesis followed an individualist methodological approach and explored whether individuals' self-perceptions and beliefs can be considered possible drivers of or impediments to institutional change.

In order to achieve its objective, this thesis relied on a theoretical framework based on elements from cognitive science and institutional economics and claimed that this can can be useful to understand the general processes involved in shaping institutional change (or absence thereof) and generating the variety of institutions that we observe in the world today.

In the preceding chapters, I used three different methodologies to capture the essence of the complex phenomenon under scrutiny and I have analysed alternative candidate 


\section{Conclusions}

hypotheses able to explain why institutions stabilise and possibly persist. Three main themes emerged and appear as pertinent to further our understanding of the evolution of institutions and their role in economic development:

1. The rate of change of institutions is related to individual self-views and the way in which people learn.

2. The dynamics of self-efficacy generates an heterogeneous distribution in the abilities to enact institutional change. This increases the likelihood of institutional persistence.

3. The rigidity of agent's mental models affects how institutional rules evolve, and thereby economic development.

In Chapter 2, I showed that people's propensity to imitate peers depends on individual self-efficacy beliefs, i.e. those self-views people hold concerning their capacities to achieve designated outcomes. In case people see themselves as incompetent, or unable to make sense of the decisional task, they tend to imitate the actions taken by others to a greater extent and "mindlessly" follow their behaviour. This result, despite being interesting on its own in the context of the learning literature, is particularly relevant in the institutional economics domain as it poses concerns in terms of institutional change. In fact, in order for institutional change to occur, individuals should perceive that change is possible and they could achieve better outcomes under a set of alternative rules of the game. But this alone is not enough. People should also believe that they can effectively bring about the change they seek. If people have little faith in their capacities to achieve any designated goal, they will not see nor pursue any opportunity to better their conditions. This in turn implies that they will rather follow the behavioural path set by somebody else, quite possibly this being the status quo. Imitating somebody else's decision might delay or hinder the perceived need for a change in behaviour, thus institutions might not wither away but rather stabilise. This is clearly in line with [Patterson, 2010] idea according to which imitation and intergenerational learning favours the institutional reproduction.

Chapter 2 establishes that a clear relation between self-efficacy beliefs, learning and possibly institutional inertia exists. This represents a first step but clearly does not fully uncover whether the low self-efficacy beliefs can be considered a clear obstacle to the institutional evolution. For this reason, in Chapter 3 I elaborated a simple model which takes into account the dynamics of self-efficacy and analyses the long-term institutional evolution and collective welfare. I showed that the endogenous dynamics of self-efficacy beliefs generate an unequal distribution of capabilities to enact change. This, ultimately increase the likelihood of institutional persistence and reduces collective welfare. Few 
'trendsetters' alone are unable to bring about a complete behavioural shift and lead to the adoption of a new, more advantageous institutional rule. This model proves particularly useful in highlighting how the feedback on self-efficacy can generate specific institutional outcomes. This would not be necessarily evident using an empirical approach given the limited data availability and time-span possibly analysed.

Despite the interesting results obtained, it worth acknowledging that Chapter 3 treats institutional change in a highly abstract manner. Thus, in order to assess whether selfviews affect the functioning and evolution of institutions having great long-run economic consequences, Chapter 4 takes an empirical approach. In this chapter I posit that highly prescriptive and immutable mental models are likely to reduce people's perceived freedom of choice and ability to control the outcome of their actions. I use two different measures to proxy mental models' stickiness and, independently from the measure considered, it appears that countries wherein people feel they have high freedom of choice and control tend also to have higher rule of law. Most of the variation is, not surprisingly, crosssectional. I also test whether mental models and institutions exert an independent effect on economic performance. A pooling model shows that mental models matter for growth and their effect is independent from institutions. In order to tackle possible endogeneity issues, I use cross-sectional IV regressions and present some evidence that mental models condition growth. This study promotes a richer view of economic development: economic prosperity depends on institutional quality but also possibly on the perceived degree of freedom of choice and control that the individuals feel.

This thesis shows that psychological factors, specifically people's expectations and beliefs about their capabilities to enact change, affect the choice on whether to change or mindlessly follow the standing institutional rules. In order to understand institutional evolution, it is of fundamental importance to consider the way in which humans make decisions and cope with individual self-views and mental models' prescriptions. I used a wide range of methods to stress the role that boundedly rational individuals play in determining institutional change (or absence thereof). Multiple methodological approaches, despite their intrinsic limitations, allowed me to propose a micro-founded view of the institutional evolution.

It is worth acknowledging that when it comes to empirically explaining specific cases of institutional change or persistence, it will take more than a set of hypotheses and an intellectually plausible framework. Further research will surely benefit from the increased data availability. In order to test the framework validity, it would be of primary importance to obtain long time series on wide variety of self-views. The advent of big-data or large scale surveys could be indeed useful to achieve this goal, and should be considered a priority. Additionally, including a wide the set of individual beliefs in this framework 


\section{Conclusions}

is of great importance. This thesis has considered self-efficacy beliefs and individually perceived abilities to carry out certain actions as the grassroots element. Indeed, there are many other self-views that concur in affecting people's interpretations of the "rules of the game" and thus their intention to change them. Self-esteem, self-control, willpower, self-disappointment and confidence are known to determine how individuals construe their self-image and identity as well as how they portray the environment wherein they act. As a consequence, these individual beliefs might as well affect the likelihood of people's engagement into trying to change institutions. Detecting their potential role in the evolution of the institutions would be a interesting avenue for future research. Moreover, including power structures and dynamics into the framework would also be extremely beneficial. In fact, it is well-known that various groups compete to protect their interests. The divergence between the goals of the groups as well as that between the group and its individual members is to be considered a crucial obstacle to institutional evolution. Therefore, analysing how groups dynamics, power distribution and sources interact with personal individuals' self-views is indeed a valuable research objective.

As a whole, this thesis provides an insightful way to analyse institutional evolution or the absence thereof. First, my work highlights how psychology, and cognitive science can, in general, provide useful tools to give a better foundations to models of economic behaviour. The importance of this is by now well established. Adam Smith noticed that economic behaviour, like any other behaviour, emerges from rational considerations as well as from individual sentiments and emotions. In 2002, Daniel Kahneman a well-known psychologists whose research goal is to uncover the 'logic of the irrational' was awarded a Nobel Prize in economics. In October 2017, Richard Thaler received the Nobel Prize for his work on, among other things, confidence, information processing and decision-making. All these are clear signs that the relation between economics and psychology is becoming stronger. Nevertheless, a lot remains to be done to fully uncover the implications of individual beliefs and self-views for economic decision-making. This thesis moves in this direction and possibly represents a concrete example of how solid psychology findings could contribute to our understanding to economic phenomena.

Additionally this thesis might deepen our understanding of economic development processes. For most economists, good institutions are the key factor able to explain the current wealth of nations. Most of this literature, though, focuses its attention to macro aspects. My thesis provides a micro-founded framework to analyse current institutional arrangements. In fact, all preceding chapters show that self-efficacy beliefs affect current and future actions as well as the desirability of change. Thus, self-efficacy could be responsible for productive investment decisions which prove important to promote development. Micro-level evidence shows that, in the development context, people often fail 
to invest despite the likelihood of obtaining positive returns. Two main complementary explanations have been advanced. Investments might fail to occur because people believe that returns from investments are not easily appropriable, and/or because information is fragmented. These explanations identify external constraints as the key determinant of investment decisions. My thesis rather proposes a richer view according to which, in an evolutionary manner, external constraints blend with people's self-perceptions and choices: the decision to invest and ameliorate one's wellbeing depends on the extent to which agents feel in control of their life and destiny as a consequence of their past experiences. Agents might abstain from making investments that would increase their wellbeing simply because they reckon, in a boundedly rational manner, that these investments are either not feasible or would not significantly change their fate. As a consequence, policy interventions should be targeted to increase poor people's understanding of the opportunities they have. Tanguy et al. [2014] found that displaying short documentaries in which agents from similar economic backgrounds tell their stories about how they managed in ameliorating their conditions improved people's aspirations as well as their score in psychological measures such as locus of control. Thus, "edutainment" interventions represent viable options to increase people's forward-looking behaviour and possibly engagement in institutional change. 



\section{Bibliography}

Acemoglu, D., S. Johson, and A. Robinson (2001). The colonial origins of comparative development: An empirical investigation. American Economic Review 91(5), 13691401.

Acemoglu, D., S. Naidu, P. Restrepo, and J. A. Robinson (2014). Democracy does cause growth. Technical report, National Bureau of Economic Research.

Acemoglu, D. and J. A. Robinson (2006). Economic origins of dictatorship and democracy. Cambridge University Press.

Acemoglu, D. and J. A. Robinson (2008). The persistence and change of institutions in the americas. Southern Economic Journal 75(2), 282-299.

Acemoglu, D. and R. Robinson (2012). Why nations fail. The Origins of Power, Prosperity and Poverty. Crown Business, New York.

Ahuja, K., M. van der Schaar, and W. Zame (2017). A theory of individualism, collectivism and economic outcomes. Mimeo.

Ajzen, I. (1991). The theory of planned behavior. Organizational Behavior and Human Decision Processes 50(2), 179-211.

Ajzen, I. (2002). Perceived behavioral control, self-efficacy, locus of control, and the theory of planned behavior. Journal of Applied Social Psychology 32(4), 665-683.

Alesina, A. and P. Giuliano (2015). Culture and institutions. Journal of Economic Literature 53(4), 898-944.

Alesina, A., P. Giuliano, and N. Nunn (2013). On the origins of gender roles: Women and the plough. The Quarterly Journal of Economics 128(2), 469-530.

Alicke, M. D., M. L. Klotz, D. L. Breitenbecher, T. J. Yurak, and D. S. Vredenburg (1995). Personal contact, individuation, and the better-than-average effect. Journal of Personality and Social Psychology 68(5), 804-825. 
Alós-Ferrer, C. (2004). Cournot versus walras in dynamic oligopolies with memory. International Journal of Industrial Organization 22(2), 193-217.

Alós-Ferrer, C. and K. H. Schlag (2009). Imitation and learning. In P. Anand, P. K. Pattanaik, and C. Puppe (Eds.), The handbook of rational and social choice : an overview of new foundations and applications, pp. 13-43. Oxford: Oxford University Press.

Amable, B. (2000). Institutional complementarity and diversity of social systems of innovation and production. Review of International Political Economy 7(4), 645-687.

Anderson, L. R. and C. A. Holt (1997). Information cascades in the laboratory. American Economic Review 87(5), 847-862.

Aoki, M. (2001). Toward a comparative institutional analysis. Cambridge (Massachusetts):MIT press.

Aoki, M. (2007). Endogenizing institutions and institutional changes. Journal of Institutional Economics 3(01), 1-31.

Apesteguia, J., S. Huck, and J. Oechssler (2007). Imitation-theory and experimental evidence. Journal of Economic Theory 136(1), 217 - 235.

Arthur, W. B. (1989). Competing technologies, increasing returns, and lock-in by historical events. The Economic Journal $99(394)$, 116-131.

Arthur, W. B. (1991). Designing economic agents that act like human agents: A behavioral approach to bounded rationality. American Economic Review 81 (2), 353-359.

Arthur, W. B. (1993). On designing economic agents that behave like human agents. Journal of Evolutionary Economics 3(1), 1-22.

Arthur, W. B. (1994). Increrasing returns and path dependence in the economy. Ann Arbor: University of Michigan Press.

Axelrod, R. (1986). An evolutionary approach to norms. American Political Science Review 80(04), 1095-1111.

Bandura, A. (1986). Social foundations of thought and action. New York: Prentice-Hall.

Bandura, A. (1989). Human agency in social cognitive theory. American psychologist $44(9), 1175-1184$.

Bandura, A. (1997). Self-efficacy: The exercise of control. New York : W.H. Freeman. 
Bandura, A. (2001). Social cognitive theory: An agentic perspective. Annual Review of Psychology 52(1), 1-26.

Banerjee, A. V. (1992). A simple model of herd behavior. The Quarterly Journal of Economics 107(3), 797-817.

Banks, J., M. Olson, and D. Porter (1997). An experimental analysis of the bandit problem. Economic Theory 10(1), 55-77.

Bao, T. (2012). Experiments on heterogeneous expectations and switching behavior. $\mathrm{Ph}$. D. thesis, Amsterdam School of Economics Research Institute.

Barber, B. and T. Odean (1999). Do investors trade too much? American Economic Review 89(5), 1279-1298.

Baumeister, R. F. and B. Bushman (2010). Social psychology and human nature. Cengage Learning.

Bechara, A., H. Damasio, D. Tranel, and A. R. Damasio (1997). Deciding advantageously before knowing the advantageous strategy. Science 275 (5304), 1293-1295.

Belloc, M. and S. Bowles (2013). The persistence of inferior cultural-institutional conventions. American Economic Review 103(3), 93-98.

Bénabou, R. (2015). The economics of motivated beliefs. Revue d'Économie Politique 125(5), 665-685.

Bénabou, R. and J. Tirole (2002). Self-confidence and personal motivation. The Quarterly Journal of Economics 117(3), 871-915.

Bénabou, R. and J. Tirole (2004). Willpower and personal rules. Journal of Political Economy 112(4), 848-886.

Bénabou, R. and J. Tirole (2016). Mindful economics: The production, consumption, and value of beliefs. The Journal of Economic Perspectives 30(3), 141-164.

Bernard, T., S. Dercon, A. S. Taffesse, et al. (2011). Beyond fatalism: an empirical exploration of self-efficacy and aspirations failure in ethiopia. Centre for Study of African Economies Working Paper 3, 1-24.

Bernheim, B. D. (1994). A theory of conformity. Journal of Political Economy 102(5), $841-877$. 
Bicchieri, C. (2005). The grammar of society: The nature and dynamics of social norms. New York, NY, US: Cambridge University Press.

Bicchieri, C. (2017). Norms in the Wild: How to Diagnose, Measure, and Change Social Norms. New York: Oxford University Press.

Bikhchandani, S., D. Hirshleifer, and I. Welch (1992). A theory of fads, fashion, custom, and cultural change as informational cascades. Journal of Political Economy 100(5), 992-1026.

Blume, L. and S. Durlauf (2003). Equilibrium concepts for social interaction models. International Game Theory Review 5(03), 193-209.

Blume, L. and S. N. Durlauf (2001). The interactions-based approach to socioeconomic behavior. Social Dynamics, 15-44.

Blume, L. E. (1995). The statistical mechanics of best-response strategy revision. Games and Economic Behavior 11(2), 111-145.

Brock, W. and C. Hommes (1997). A rational route to randomness. Econometrica 65(5), 1059-1096.

Brock, W. A. and S. N. Durlauf (1999). A formal model of theory choice in science. Economic Theory 14(1), 113-130.

Brock, W. A. and S. N. Durlauf (2001a). Discrete choice with social interactions. The Review of Economic Studies 68(2), 235-260.

Brock, W. A. and S. N. Durlauf (2001b). Interactions-based models. In J. J. Heckman and E. Leamer (Eds.), Handbook of Econometrics, Volume 5, pp. 3297-3380. Elsevier.

Burke, C. J., P. N. Tobler, M. Baddeley, and W. Schultz (2010). Neural mechanisms of observational learning. Proceedings of the National Academy of Sciences 107(32), 14431-14436.

Burnham, K. P. and D. R. Anderson (1998). Model selection and multimodel inference: a practical information-theoretic approach. Springer Science \& Business Media.

Bush, R. R. and F. Mosteller (1951). A mathematical model for simple learning. Psychological Review 58(5), 313-323.

Bush, R. R. and F. Mosteller (1953). A stochastic model with applications to learning. The Annals of Mathematical Statistics 24(4), 559-585. 
Calvert, R. (1995). Rational actors, equilibrium, and social institutions. In J. Night and I. Sened (Eds.), Explaining social institutions, pp. 57-95. Ann Arbor: University of Michigan Press.

Camerer, C. (2003). Behavioral game theory : experiments in strategic interaction. Princeton, N.J.: Princeton University Press.

Camerer, C. and D. Lovallo (1999). Overconfidence and excess entry: An experimental approach. American Economic Review 89(1), 306-318.

Cross, J. G. (1973). A stochastic learning model of economic behavior. The Quarterly Journal of Economics 87(2), 239-266.

David, P. A. (1985). Clio and the economics of qwerty. American Economic Review 75 (2), 332-337.

David, P. A. (1994). Why are institutions the carriers of history ĂŹ?: Path dependence and the evolution of conventions, organizations and institutions. Structural Change and Economic Dynamics 5(2), 205-220.

Denzau, A. T. and D. C. North (1994). Shared mental models: ideologies and institutions. Kyklos $47(1), 3-31$.

Diks, C. and R. Van Der Weide (2005). Herding, a-synchronous updating and heterogeneity in memory in a cbs. Journal of Economic Dynamics and Control 29(4), 741-763.

DiMaggio, P. (1997). Culture and cognition. Annual Review of Sociology 23(1), 263-287.

Durlauf, S. N. (1993). Nonergodic economic growth. The Review of Economic Studies $60(2), 349-366$.

Easterly, W. and R. Levine (2003). Tropics, germs, and crops: how endowments influence economic development. Journal of Monetary Economics 50(1), 3-39.

Efferson, C., P. J. Richerson, R. McElreath, M. Lubell, E. Edsten, T. M. Waring, B. Paciotti, and W. Baum (2006). Learning, productivity, and noise: an experimental study of cultural transmission on the bolivian altiplano. Evolution and Human Behavior 28(1), 11-17.

Ellison, G. and D. Fudenberg (1993). Rules of thumb for social learning. Journal of Political Economy 101(4), 612-643. 
Erev, I. and A. E. Roth (1998). Predicting how people play games: Reinforcement learning in experimental games with unique, mixed strategy equilibria. American Economic Review 88(4), 848-881.

Erev, I. and A. E. Roth (2007). Multi-agent learning and the descriptive value of simple models. Artificial Intelligence 171(7), $423-428$.

Fehr, E. and K. Hoff (2011). Introduction: Tastes, castes and culture: the influence of society on preferences*. The Economic Journal 121(556), 396-412.

Fernández, R. (2008). Culture and economics. The New Palgrave Dictionary of Economics 2, 333-340.

Fernández, R. (2011). Does culture matter? In J. Benhabib, A. Bisin, and M. O. Jackson (Eds.), Handbook of Social Economics. Elsevier.

Fortin, B., G. Lacroix, and M.-C. Villeval (2007). Tax evasion and social interactions. Journal of Public Economics 91(11), 2089-2112.

Fudenberg, D., R. Iijima, and T. Strzalecki (2015). Stochastic choice and revealed perturbed utility. Econometrica 83(6), 2371-2409.

Gagliardi, F. (2008). Institutions and economic change: A critical survey of the new institutional approaches and empirical evidence. The Journal of Socio-Economics 37(1), 416-443.

Gans, N., G. Knox, and R. Croson (2007). Simple models of discrete choice and their performance in bandit experiments. Manufacturing \& Service Operations Management 9(4), 383-408.

Gigerenzer, G. (2004). Mindless statistics. The Journal of Socio-Economics 33(5), 587606.

Gigerenzer, G., S. Krauss, and O. Vitouch (2004). The null ritual. In D.Kaplan (Ed.), The Sage Handbook of Quantitative Methodology for the Social Sciences, pp. 391-408. Thousand Oaks, CA: Sage.

Gittins, J. C. (1979). Bandit processes and dynamic allocation indices. Journal of the Royal Statistical Society. Series B (Methodological) 41(2), 148-177.

Gorodnichenko, Y. and G. Roland (2017). Culture, institutions and the wealth of nations. Review of Economics and Statistics 99(3), 402-416. 
Greif, A. (1993). Contract enforceability and economic institutions in early trade: The maghribi traders' coalition. American Economic Review 83(3), 525-548.

Greif, A. (2006). Institutions and the path to the modern economy: Lessons from medieval trade. Cambridge (England): Cambridge University Press.

Greif, A. and C. Kingston (2011). Institutions: Rules or equilibria? In N. Schofield and G. Caballero (Eds.), Political economy of institutions, democracy and voting, pp. 13-43. Berlin: Springer.

Greif, A. and G. Tabellini (2010). Cultural and institutional bifurcation: China and europe compared. American Economic Review 100(2), 135-140.

Guala, F. (2016). Understanding Institutions: The Science and Philosophy of Living Together. Princeton University Press.

Guiso, L., P. Sapienza, and L. Zingales (2006). Does culture affect economic outcomes? The Journal of Economic Perspectives 20(2), 23-48.

Guiso, L., P. Sapienza, and L. Zingales (2009). Cultural biases in economic exchange? The Quarterly Journal of Economics 124(3), 1095-1131.

Hayek, F. (1973). Law, legislation and liberty: a new statement of the liberal principles of justice and political economy. London: Routledge.

Herrnstein, R. J. (1961). Relative and absolute strength of response as a function of frequency of reinforcement. Journal of the Experimental Analysis of Behavior 4, 267272.

Hiroto, D. S. and M. E. Seligman (1975). Generality of learned helplessness in man. Journal of Personality and Social Psychology 31(2), 311-327.

Hodgson, G. M. (2007). Institutions and individuals: interaction and evolution. Organization Studies 28(1), 95-116.

Hoff, K. and P. Pandey (2006). Discrimination, social identity, and durable inequalities. American Economic Review 96(2), 206-211.

Hoff, K. and P. Pandey (2014). Making up people: ĂŤthe effect of identity on performance in a modernizing society. Journal of Development Economics 106, 118-131.

Hoff, K. and J. E. Stiglitz (2010). Equilibrium fictions: A cognitive approach to societal rigidity. American Economic Review 100(2), 141-146. 
Hofstede, G. (2003). Culture's consequences: Comparing values, behaviors, institutions and organizations across nations. Sage publications.

Hommes, C. H. (1994). Dynamics of the cobweb model with adaptive expectations and nonlinear supply and demand. Journal of Economic Behavior $\&$ Organization 24(3), 315-335.

Huck, S., H.-T. Normann, and J. Oechssler (1999). Learning in cournot oligopoly-an experiment. The Economic Journal 109(454), 80-95.

Hurley, S. L. and N. Chater (2005). Perspectives on Imitation: from neuroscience to social science. Cambridge, Mass. ; London : MIT.

Hwang, S. H., S. Naidu, and S. Bowles (2013). Institutional persistence and change: An evolutionary approach. Unpublished.

Ioannides, Y. M. and L. Datcher Loury (2004). Job information networks, neighborhood effects, and inequality. Journal of Economic Literature 42(4), 1056-1093.

Judge, T. A. and J. E. Bono (2001). Relationship of core self-evaluations traits - selfesteem, generalized self-efficacy, locus of control, and emotional stability - ǍTwith job satisfaction and job performance: A meta-analysis. Journal of Applied Psychology $86(1), 80-92$.

Kahneman, D. (2003). Maps of bounded rationality: Psychology for behavioral economics. American Economic Review 93(5), 1449-1475.

Kahneman, D. (2011). Thinking, fast and slow. London: Macmillan.

Kaufmann, D. and A. Kraay (2002). Growth without governance. World Bank Policy Research Working Paper (2928).

Kingston, C. and G. Caballero (2009). Comparing theories of institutional change. Journal of Institutional Economics 5(2), 151-180.

Klein, D. C., E. Fencil-Morse, and M. E. Seligman (1976). Learned helplessness, depression, and the attribution of failure. Journal of Personality and Social Psychology 33(5), $508-516$.

Libecap, G. D. (1989). Contracting for property rights. Cambridge (England): Cambridge University Press.

Luce, R. (1959). Individual Choice Behavior a Theoretical Analysis. New York: Wiley. 
Mahoney, J. (2000). Path dependence in historical sociology. Theory and society 29(4), $507-548$.

Malmendier, U. and G. Tate (2005). Ceo overconfidence and corporate investment. The Journal of Finance 60(6), 2661-2700.

Mantzavinos, C., D. C. North, and S. Shariq (2004). Learning, institutions, and economic performance. Perspectives on Politics 2(01), 75-84.

Markus, H. (1977). Self-schemata and processing information about the self. Journal of personality and social psychology 35(2), 63-78.

Markus, H. R. and S. Kitayama (1991). Culture and the self: Implications for cognition, emotion, and motivation. Psychological review 98(2), 224.

Martin, R. (2010). Rethinking regional path-dependence: beyond lock-in to evolution. Economic Geography 86(1), 1-27.

Martinko, M. J. and W. L. Gardner (1982). Learned helplessness: An alternative explanation for performance deficits. Academy of Management Review 7(2), 195-204.

Matêjka, F. and A. McKay (2015). Rational inattention to discrete choices: A new foundation for the multinomial logit model. American Economic Review 105(1), 27298.

McElreath, R., A. V. Bell, C. Efferson, M. Lubell, P. J. Richerson, and T. Waring (2008). Beyond existence and aiming outside the laboratory: estimating frequency-dependent and pay-off-biased social learning strategies. Philosophical Transactions of the Royal Society of London B: Biological Sciences 363(1509), 3515-3528.

McElreath, R., M. Lubell, P. J. Richerson, T. M. Waring, W. Baum, E. Edsten, C. Efferson, and B. Paciotti (2005). Applying evolutionary models to the laboratory study of social learning. Evolution and Human Behavior 26 (6), 483-508.

Merlo, A. and A. Schotter (2003). Learning by not doing: an experimental investigation of observational learning. Games and Economic Behavior 42(1), 116-136.

Miller, N. E. and J. Dollard (1941). Social learning and imitation. New Haven, CT: Yale University Press.

Nax, H. H., M. N. Burton-Chellew, S. A. West, and H. P. Young (2016). Learning in a black box. Journal of Economic Behavior \&3 Organization 127, 1-15. 
Nelson, R. and S. Winter (1982). An evolutionary theory of economic change. Cambridge, MA: Harvard Business School Press.

Nicolle, A., M. Symmonds, and R. Dolan (2011). Optimistic biases in observational learning of value. Cognition 119(3), 394-402.

North, D. C. (1990). Institutions, institutional change and economic performance. Cambridge (England):Cambridge university press.

North, D. C. (1994). Economic performance through time. American Economic Review 84 (3), 359-368.

North, D. C. (2003). The new institutional economics and third world development. In J. Harriss, J. Hunter, and C. Lewis (Eds.), The new institutional economics and third world development, pp. 17-27. London: Routledge.

Offerman, T., J. Potters, and J. Sonnemans (2002). Imitation and belief learning in an oligopoly experiment. The Review of Economic Studies 69(4), 973-997.

Offerman, T. and A. Schotter (2009). Imitation and luck: An experimental study on social sampling. Games and Economic Behavior 65(2), 461 - 502.

Offerman, T. and J. Sonnemans (1998). Learning by experience and learning by imitating successful others. Journal of Economic Behavior \& Organization 34(4), 559-575.

Olson, M. (1965). The logic of collective action. Public goods and the theory of groups. Cambridge (Massachusetts):Harvard University Press.

Ostrom, E. (2005). Understanding institutional diversity. Princeton: Princeton university press.

Overmier, J. B. and M. E. Seligman (1967). Effects of inescapable shock upon subsequent escape and avoidance responding. Journal of Comparative and Physiological Psychology $63(1), 28-33$.

Patterson, O. (2010). The mechanism of cultural reproduction: explaining the puzzle of persistence. In J. R. Hall, L. Grindstaff, and M.-C. Lo (Eds.), Handbook of Cultural Sociology, pp. 139-151. Oxford: Routledge.

Pingle, M. and R. H. Day (1996). Modes of economizing behavior: Experimental evidence. Journal of Economic Behavior \& Organization 29(2), 191-209.

Rabow, J., S. L. Berkman, and R. Kessler (1983). The culture of poverty and learned helplessness: A social psychological perspective. Sociological Inquiry 53(4), 419-434. 
Rendell, L., R. Boyd, D. Cownden, M. Enquist, K. Eriksson, M. W. Feldman, L. Fogarty, S. Ghirlanda, T. Lillicrap, and K. N. Laland (2010). Why copy others? insights from the social learning strategies tournament. Science 328(5975), 208-213.

Rodrik, D., A. Subramanian, and F. Trebbi (2004). Institutions rule: the primacy of institutions over geography and integration in economic development. Journal of Economic Growth 9(2), 131-165.

Roland, G. (2008). Fast-moving and slow-moving institutions. In Institutional Change and Economic Behaviour, pp. 134-159. Springer.

Roth, A. E. and I. Erev (1995). Learning in extensive-form games: Experimental data and simple dynamic models in the intermediate term. Games and Economic Behavior 8(1), $164-212$.

Schlag, K. H. (1998). Why imitate, and if so, how? Journal of Economic Theory 78(1), $130-156$.

Schotter, A. (1981). The economic theory of social institutions. Cambridge (England): Cambridge University Press.

Schwarzer, R., J. Bäßler, P. Kwiatek, K. Schröder, and J. X. Zhang (1997). The assessment of optimistic self-beliefs: comparison of the german, spanish, and chinese versions of the general self-efficacy scale. Applied Psychology 46(1), 69-88.

Schwarzer, R. and M. Jerusalem (1995). Generalized self-efficacy scale. In J. Weinman, S. Wright, and M. Johnston (Eds.), Measures in health psychology: A users portfolio. Causal and control beliefs, pp. 35-37. Windsor, UK: NFER-NELSON.

Seligman, M. E., L. Y. Abramson, A. Semmel, and C. Von Baeyer (1979). Depressive attributional style. Journal of Abnormal Psychology 88(3), 242-247.

Selten, R. and A. Ostmann (2000). Imitation equilibrium. Technical report, Bonn Econ Discussion Papers.

Sen, A. (2001). Development as Freedom. Oxford: Oxford Paperbacks.

Simon, H. A. (1955). A behavioral model of rational choice. The Quarterly Journal of Economics 69(1), 99-118.

Simon, H. A. (1959). Theories of decision-making in economics and behavioral science. American Economic Review 49(3), 253-283. 
Spolaore, E. and R. Wacziarg (2009). The diffusion of development. The Quarterly Journal of Economics 124(2), 469-529.

Staiger, D., J. H. Stock, et al. (1997). Instrumental variables regression with weak instruments. Econometrica 65(3), 557-586.

Streeck, W. and K. A. Thelen (2005). Institutional change in advanced political economies. In W. Streeck and K. A. Thelen (Eds.), Beyond continuity, pp. 1-39. Oxford University Press.

Svenson, O. (1981). Are we all less risky and more skillful than our fellow drivers? Acta Psychologica 47(2), 143-148.

Szirmai, A. (2005). The dynamics of socio-economic development: an introduction. Cambridge (England): Cambridge University Press.

Tanguy, B., S. Dercon, K. Orkin, and A. S. Taffesse (2014). The future in mind: Aspirations and forward-looking behaviour in rural ethiopia. CEPR Discussion Papers.

Thorndike, E. (1898). Some experiments on animal intelligence. Science 7(181), 818-824.

Tomasello, M., M. Davis-Dasilva, L. Camak, and K. Bard (1987). Observational learning of tool-use by young chimpanzees. Human Evolution 2(2), 175-183.

Unicef (2016). Female genital mutilation/cutting: a global concern. Technical report, New York: UNICEF.

Vega-Redondo, F. (1997). The evolution of walrasian behavior. Econometrica 65(2), 375-384.

Vostroknutov, A., L. Polonio, and G. Coricelli (2017). Observational learning and intelligence. Technical Report Research Paper No. 17-05, USC-INET.

Williamson, O. E. (2000). The new institutional economics: taking stock, looking ahead. Journal of Economic Literature 38(3), 595-613.

Young, H. (1998a). Individual strategy and social institutions: an evolutionary theory of institutions. Princeton: Princeton University Press.

Young, H. P. (1998b). Conventional contracts. The Review of Economic Studies 65(4), $773-792$.

Young, H. P. (2001). Individual strategy and social structure: An evolutionary theory of institutions. Princeton University Press. 
Young, H. P. (2011). The dynamics of social innovation. Proceedings of the National Academy of Sciences 108(Supplement 4), 21285-21291.

Young, H. P. (2015). The evolution of social norms. Annual Review of Economics \%, $359-387$.

Young, H. P. and M. A. Burke (2001). Competition and custom in economic contracts: a case study of illinois agriculture. American Economic Review 91(3), 559-573.

Zeppini, P. (2015). A discrete choice model of transitions to sustainable technologies. Journal of Economic Behavior $\&$ Organization 112, 187-203. 



\section{About the Author}

Stefania earned a Bachelor in Development Economics (summa cum laude) and a Master in Economics (summa cum laude) from the University of Florence in Italy. From 2009 to 2010, she worked as a research assistant at the European University Institute and at the University of Florence. She next moved to Geneva to work for the UN International Labour Office and then to Rome as a consultant at the Italian Ministry of Economics and Finance. She joined UNU-MERIT and the School of Business and Economics of Maastricht University in September 2012. In the course of her Ph.D studies, Stefania was a tutor of a Master level course on "Intellectual Property Rights in Network Societies". She lectured on behavioural economics and experimental methods in the Master's programme in Public Policy and Human Development and she also supervised eleven bachelor thesis students on a wide variety of topics. From October 2016 to August 2017 she was a Junior Visiting Scholar at Nuffield College at the University of Oxford. Currently, Stefania holds a stipendiary lecturer position in economics at St. Peter's College in Oxford and a Research Associate position in behaviour, finance and social statistics at the Smith School of Enterprise and the Environment at the University of Oxford. 



\section{Valorization Addendum}

In line with Article 23 of the regulation governing the attainment of the doctoral degree at Maastricht University, this section highlights the valorisation opportunities of this doctoral thesis. The goal of this section is to describe how the knowledge resulting from this research project can be valuable for society and particularly for policy makers and academics.

Over the last 50 years, academics and policy makers have focused a great deal of their attention to how to promote sustainable development and more specifically alleviate poverty and improve financial productive decisions. Building upon the growing empirical evidence provided by the academic literature, policy makers escaped the lure of topdown approaches and came to realise that local institutions play a crucial role in this. They represent one of the key predictors of development. In fact, whereas positive and effective institutions lead to societal wealth and prosperity, ineffective and negative ones have proven to curtail growth and development. As a consequence, a consensus has been reached: the bottom-up creation of a strong and conducive institutional environment is to be considered a priority for developing nations.

In 2016, such stance has been explicitly translated into the formulation of one of the UN Sustainable Development Goal (SDG). More specifically, the UN-SDG number 16 highlights that countries should develop effective, accountable and transparent institutions able to protect fundamental freedoms and build capacity at all levels.

This thesis embraces such priority. It studies the importance of institutions and specifically claims that understanding under which conditions institutions persist or may be amenable is imperative to effective development policy. The dissertation shows that institutional change is connected to individual behaviour, and an important aspect of this connection runs through peoples' self-efficacy, i.e. the belief that they can improve their situations through their own actions. Self-views represent the missing link between the institutional change and ultimately development outcomes.

When taking decisions including those concerning institutional change, individuals rely on a set of beliefs that they have regarding themselves and the environment they live in. These beliefs evolve through learning and experience and they affect one's motivation and perseverance. If an individual is convinced that he has little, if any, ability to improve his 
current wellbeing, i.e. he holds low self-efficacy beliefs, then he will have little incentive to gather information and evaluate the available options to ameliorate his condition. Additionally, the agent will have no reason to actively revise his self-view. As a result, his belief about his inability to bring about positive change in his life crystallises and self-perpetuates. This concretely implies that the agent will not explore the available opportunities as he is convinced that his action will not make a difference.

Due to low self-efficacy individuals may mindlessly follow the action taken by others, this in turn may lower the likelihood of exploring new and possibly valuable avenues for institutional change. Additionally, an unequal distribution of self-efficacy might lock societies in to sub-optimal institutional frameworks and consequently social change fails to diffuse. Moreover, the vertical transmission of "sticky" mental models might hold up the quest for high rule of law.

This is particularly important in poor contexts wherein people might to construct their set of beliefs prioritising some features of the environment rather than others. As a consequence, highly biased self-views might emerge and these might perpetuate poverty. Thus, given the above described dynamics, in order to meet the targets set by the UN-SDG 16, promote the endogenous creation of effective, strong and inclusive institutions and ultimately reduce poverty and foster growth, policy makers should acknowledge the importance of beliefs particularly about one's own capacity to enact change. Accordingly, for the sake of devising and implementing development policies a richer diagnosis of people's self-efficacy should be carried out.

This approach to institutional change has high potential. Its application might enhance the effectiveness of development policies and interventions. Gaining insights into people's self-efficacy is to be considered the initial step prior to the implementation of development programmes. A sound diagnosis should then be followed by actions meant to raise self-efficacy and reduce aspiration failures in ways that then allow individuals to take advantages of the available opportunities as well as to create new ones. This might increase the effectiveness of interventions which are, for instance, meant to help households to get credit or insurance, to engage in productive activities, to adopt new technological artefacts or to increase educational attainment. Improved self-efficacy might ultimately break the vicious cycle stemming from low aspiration.

The findings presented in this thesis do not apply to poor contexts only. They might also be relevant to analyse contemporary consumer decision-making in developed societies. Consider, for instance, the case in which individuals choose amongst mobile phone tariffs or pension savings schemes. In each of these cases, there exist many potential options. Some of these alternatives might not be easy to discover or even understand. This is due to the fact that at times their costs and benefits are uncertain and hard to compare. 
In these situations, consumers might be led to believe that, given the complexity of the decisional environment, they have no ability to make the right choice and fulfil their needs. As a consequence individuals' sense of efficacy reduces and likelihood of simply imitating the choices made by others or of sticking to previously made decisions increases. This can result in significant economic detriment to consumers.

Additionally, the concepts and results presented in the thesis relate to voting behaviour. In political science, political efficacy identifies citizens' faith and trust in their governments and their belief that they can understand and influence political affairs. A low sense of political efficacy entails that citizens do not believe that they can affect the actions taken by their leaders. Conversely, high political efficacy implies that citizens feel they can express their opinions and effectively shape the government course of action and possibly its re-election. The results presented in this thesis, and especially those concerned with the relation between self-efficacy and imitation as well as those related to the fact that an unequal distribution of self-efficacy might lock society into sub-optimal equilibria, could be used to explain elections' outcomes. According to these findings, low political efficacy might trigger imitation, possibly scarce deviance from the majority voting and low political turnover. Additionally, when the majority of the population has low political self-efficacy, citizens might reduce their political participation and as a consequence few vocal individuals or groups endowed with higher political efficacy might be unable to take the lead towards a shift of political preferences.

Beyond the above mentioned societal relevance, in terms of innovativeness the thesis provides a novel perspective into the academic debate on institutions. As discussed in the introduction to the book, despite a 60-years long academic debate, scarce attention had been devoted to self-views as possible impediments to change. This thesis tried to fill the gap. Concretely, this was achieved combining insights from psychology and economics. By now, the relation between economics and psychology is quite strong as testified by the work of Kahneman and Thaler who were both awarded a Nobel Prize in 2002 and 2017 respectively. However, a lot remains to be done to fully uncover the implications of individual beliefs and self-views for economic decision-making. This thesis moves in this direction. It in fact shows that the fruitful integration of solid psychology findings with robust economic models not only leads to a more comprehensive understanding of economic phenomena such as institutional change or lack thereof, but also provides scope for exploring exciting new research questions at the intersection of both disciplines.

Finally, the dissemination of the outcome of this research project is ongoing. The results have been presented to international audiences in seminars and conferences. The three main chapters of this thesis will be submitted to international peer-reviewed journals soon. 


\section{UNU-MERIT/MGSoG Dissertation Series}

2018

\section{Stefania Innocenti}

On Institutional Persistence

UNU-MERIT/MGSoG Dissertation

Series № 199

\section{Hassen Abda Wako}

Esconomic Globalization, Institutions and Development: Essays on Aid, Foreign Direct Investment and Trade UNU-MERIT/MGSoG Dissertation Series № 198

2017

\section{Hans-Erik Edsand}

Winds of Change

UNU-MERIT/MGSoG Dissertation Series № 197

\section{Ana Patricia Silva Vara}

Redressing the Gender Gap

UNU-MERIT/MGSoG Dissertation Series № 196

\section{Andrés Iván Mideros Mora}

Essays on the Economic Effects of Noncontributory Social Protection UNU-MERIT/MGSoG Dissertation Series № 195

\section{Tobias Broich}

New Actors in the Global Economy UNU-MERIT/MGSoG Dissertation Series № 194

\section{Bernard Nikaj}

From No-government to E-government UNU-MERIT/MGSoG Dissertation Series № 193

\section{Ali Safarnejad}

Prioritizing the HIV Response

UNU-MERIT/MGSoG Dissertation

Series № 192

\section{Clovis Freire}

Diversification and Structural

Economic Dynamics

UNU-MERIT/MGSoG Dissertation Series № 191

\section{Michael Verba}

Innovation and Knowledge Dynamics: Essays on the Knowledge Economy UNU-MERIT/MGSoG Dissertation Series № 190

\section{Pui Hang Wong}

The Hearts and Minds in Conflict and Peace: The Economics of

Counterinsurgency and the Psychology of Reconstruction

UNU-MERIT/MGSoG Dissertation Series № 189

\section{Brenda Yamba}

Schooling Despite All Odds: Evidence from Lesotho on Female Child Carers who Stayed in School UNU-MERIT/MGSoG Dissertation Series № 188 


\section{Sheng Zhong}

Moving towards An Energy Efficient

Future: Essays on Energy Efficiency,

Technology and Development

UNU-MERIT/MGSoG Dissertation

Series № 187

\section{Julieta Marotta}

Access to Justice and Legal

Empowerment of Victims of Domestic

Violence through Legal Organizations

in the City of Buenos Aires: A

Qualitative Empirical Legal Study

UNU-MERIT/MGSoG Dissertation

Series, № 186

\section{Andrea Franco-Correa}

On the Measurement of

Multidimensional Poverty as a Policy Tool: Empirical Applications to Chile,

Colombia, Ecuador and Peru

UNU-MERIT/MGSoG Dissertation

Series, № 185

\section{6}

\section{YesufAwel}

Insurance for Growth: Empirical

Essays on Insurance Demand and

Impacts in Africa

UNU-MERIT Dissertation Series, № 108

Tigist Mekonnen Melesse

Grow More Food using Fewer

Resources: Agricultural Technology

Adoption and Innovation Practices for

Inclusive and Sustainable

Development

UNU-MERIT Dissertation Series, № 107

\section{Eleni Yitbarek}

Getting Ahead or left Behind? Essays

on Poverty Dynamics and Social

Mobility in Africa

UNU-MERIT Dissertation Series, № 106

\section{Thuy Dieu Nguyen}

Firm-Level Theory and Evidence of

Corruption

UNU-MERIT Dissertation Series, № 105

\section{Raquel Tsukada Lehman}

Essays on Household Production with

Labor-Saving Technology

UNU-MERIT Dissertation Series,

№ 104

\section{Eva Barteková}

Multi-Problem Challenges for a

Renewable Future: Empirical Studies on Competitive Disadvantages from

Electricity Price Differentials and

Mineral Supply Risk in an Open

Economy

UNU-MERIT Dissertation Series,

№ 103

\section{Jocelyn Olivari}

Entrepreneurial Traits and Innovation:

Evidence from Chile

UNU-MERIT Dissertation Series, № 102 


\section{Muhammad Shafique}

Essays on the role of knowledge, RED, and Technology-based Firms in the

Evolution of Socio-techno-economic System

UNU-MERIT Dissertation Series, № 101

\section{Serdar Türkeli}

Governance of Innovation Policy:

Empirical Studies on Applied Political Economy by Multi-Methods Analysis UNU-MERIT Dissertation Series, № 100

\section{Ayokunu Adedokun}

Pathways to Sustainable Peace building in Divided Societies: Lessons and Experiences from Mozambique MGSoG Dissertation Series, № 75

\section{Luiz RothierBautzer}

Organizing Concurrent Engineering through ICT Platforms

Blueprinting Product Lifecycle

Management Platforms across

Disciplinary Agencies

MGSoG Dissertation Series, № 74

\section{Natalia Popova}

Migration in the Periphery of the

European Union:

Determinants of Successful and

Sustainable Labour Market Integration of Return Migrants in Albania, Egypt, Moldova and Tunisia

MGSoG Dissertations Series, № 73

\section{Richard A. Martina}

Uncertainty and Resource Constraint in the Small Island Developing States: Essays in Entrepreneurial Cognition MGSoG Dissertations Series, № 72

\section{Cécile Cherrier}

The Expansion of Basic Social Protection in Low-income Countries: An Analysis of Foreign Aid Actors' Role in the Emergence of Social Transfers in Sub-Saharan Africa MGSoG Dissertations series, № 71

\section{Paul Caldron}

The Tacit Bargain in Short-Term Medical Missions: Why U.S. physicians go and what it costs MGSoG Dissertation Series, № 70

\section{Mahmut Kobal}

Customs \& Excellence: A Comparative Approach on Administrative and Regulatory Compliance Perspectives of the EU-Turkey Customs Union MGSoG Dissertation Series, № 69

\section{Craig Loschmann}

Essays on Conflict-related Migration and Development in the Case of Afghanistan MGSoG Dissertations Series, № 68

\author{
Andrea Milan \\ Rural Livelihoods, Location and \\ Vulnerable Environments: Approaches \\ to Migration in Mountain areas of \\ Latin America \\ MGSoG Dissertation Series, № 67
}




\section{Farida Lada}

On Guarding the Welfare of Clinical

Trial Subjects While Promoting Novel

Drug Innovation

A Game Theoretical Approach

MGSoG Dissertation Series, № 66

2015

\section{Hibret Belete Maemir}

Dissecting Aggregate Productivity:

International Integration and Growth with Heterogeneous Firms

UNU-MERIT Dissertation Series, № 96

\section{Giorgio Triulzi}

Looking for the Right Path: Technology

Dynamics, Inventive Strategies and

Catching-up in the Semiconductor

Industry

UNU-MERIT Dissertation Series, № 95

\section{Abdul Baseer Qazi \\ Knowledge flows and networks in the \\ ICT sector: The case of Pakistan \\ UNU-MERIT Dissertation Series, № 94}

\section{Ajay Thutupalli}

Technology Paradigm Shifts in

Agriculture: Drivers of Sustainability

and Catch up

UNU-MERIT Dissertation Series, № 93

\section{Eduardo Urias}

Improving access to HIVIAIDS

treatment in Brazil: When are

Compulsory Licenses effective in Price Negotiations?

UNU-MERIT Dissertation Series, № 92

\section{Francesca Guadagno}

Why have so few Countries

Industrialised?

UNU-MERIT Dissertation Series, № 91

\section{Daniel Opolot}

The Evolution of Beliefs and Strategic Behaviour

UNU-MERIT Dissertation Series, № 90

\author{
Alejandro Lavopa \\ Structural Transformation and \\ Economic Development: Can \\ Development Traps be Avoided \\ UNU-MERIT Dissertation Series, \\ № 89
}

\section{Jinjin Zhao}

Urban water management reform: The Case of China

UNU-MERIT Dissertation Series, № 88

\section{Simona Vezzoli}

Borders, Independence and Postcolonial Ties: the Role of the State in Caribbean Migration MGSoG Dissertation Series, № 65 


\section{Silvia Consuelo Gómez Soler}

Civil Conflict and Education: How

Does Exposure to Civil Conflict Affect

Human Capital Accumulation?

Evidence from Standardized Exit

Exams in Colombia

MGSoG Dissertation Series, № 64

\section{Paula Nagler}

Occupational Choice in the Developing

World

MGSoG Dissertation Series, № 63

\section{Jasmin Kientzel}

Determinants of Professional

Commitment to Environmental

Sustainability

MGSoG Dissertation Series, № 62

\section{Mehmet GüneyCelbiş}

Regional Policies: Convergence, Trade, and the Allocation of Public Capital MGSoG Dissertation Series, № 61

\section{Florian Henning}

Living Up to Standard:

Interoperability Governance and

Standards Adoption in Government

Information Networks

MGSoG Dissertation Series, № 60

Niels P. Groen

The Never-Ending Project

Understanding E-Government Project

Escalation

MGSoG Dissertation Series, № 59

\section{Derek Copp}

Teacher-Based Reactivity to Provincial

Large-scale Assessment in Canada

MGSoG Dissertation Series, № 58

\section{Michaella Vanore}

Family-Member Migration and the

Psychosocial Health Outcomes of

Children in Moldova and Georgia

MGSoG Dissertation Series, № 57

\section{Sonja Fransen}

The Economic and Social Effects of

Remittances and Return Migration in

Conflict-Affected Areas: The Case of

Burundi

MGSoG Dissertation Series, № 56

\section{Ibrahim Khalil Conteh}

The Impact of Floods on Primary

School Education in Zambia

MGSoG Dissertation Series, № 55

\section{Richard Bluhm}

Growth Dynamics and Development Essays in Applied Econometrics and Political Economy

MGSoG Dissertation Series, № 54

\section{Nevena P. Zhelyazkova}

Work-Family Reconciliation and Use of Parental Leave in Luxembourg:

Empirical Analysis of Administrative Records

MGSoG Dissertation Series, № 53

\section{4}

\section{Dirk Crass}

The Impact of Brands on Innovation and Firm Performance: Empirical Evidence from Germany UNU-MERIT Dissertation Series, № 87 


\section{Samyukta Bhupatiraju}

The Geographic Dimensions of Growth and Development

UNU-MERIT Dissertation Series, № 86

\section{François Lafond}

TheEvolution of Knowledge Systems UNU-MERIT Dissertation Series, № 85

\section{Annalisa Primi}

Promoting Innovation in Latin

America: What Countries Have

Learned (and What They Have Not) in Designing and Implementing

Innovation and Intellectual Property

Policies

UNU-MERIT Dissertation Series, № 84

\section{Fatoumata Lamarana Diallo}

Evaluation of Meal and Deworming Programs for Primary Schools in Rural Senegal

UNU-MERIT Dissertation Series, № 83

\section{Sachin Kumar Badkas}

Metachoice and Metadata: Innovating with Environmental Policy Analysis in Europe

MGSoG Dissertation Series, № 52

\section{Irina S. Burlacu}

An Evaluation of Tax-Benefit Systems Impact on the Welfare of Frontier Worker:

The Case of Luxembourg and Belgium MGSoG Dissertation Series, № 51

\section{ÖzgeBilgili}

Simultaneity in Transnational

Migration Research: Links Between

Migrants' Host and Home Country

Orientation

MGSoG Dissertation Series, № 50

\section{Yulia Privalova Krieger}

Reshaping the Big Agenda:

Transnational Politics and Domestic

ResistanceFinancial crisis and social protection reform in Bosnia and

Herzegovina

MGSoG Dissertation Series, № 49

\section{Marieke van Houte}

Moving Back or Moving Forward?

Return migration after Conflict

MGSoG Dissertation Series, № 48

\section{Oxana Slobozhan}

Global Governance in the Management of Natural Resources: The Case of the

Extractive Industries Transparency Initiative (EITI)

MGSoG Dissertation Series, № 47

\section{Luis Bernardo Mejia Guinand}

The Changing Role of the Central

Planning Offices in Latin America: A

Comparative Historical Analysis

Perspective (1950-2013)

MGSoG Dissertation Series, № 46

\section{Cheng Boon Ong}

Ethnic Segregation in Housing, Schools and Neighbourhoods in the Netherlands

MGSoG Dissertation Series, № 45 


\section{Luciana V. Cingolani}

Bureaucracies for Development:

Oxymoron or Reality? Studies on State

Capacity in Challenging Governance

Contexts

MGSoG Dissertation Series, № 44

\section{Carlos Cadena Gaitán}

Green Politics in Latin American

Cities - Sustainable Transport Agendas

MGSoG Dissertation Series, № 43

\section{Katie Kuschminder}

Female Return Migration and

Reintegration Strategies in Ethiopia

MGSoG Dissertation Series, № 42

\section{Metka Hercog}

Highly-Skilled Migration and New

Destination Countries

MGSoG Dissertation Series, № 41

\section{Margaret Agaba Rugadya}

Can Remittances Influence the Tenure and Quality of Housing in Uganda?

MGSoG Dissertation Series, № 40

\section{IlireAgimi}

New Governance Under Limited

Statehood: The Case of Local

Government Reform in Kosovo

MGSoG Dissertation Series, № 39

2013

\section{Anant Kamath}

Information Sharing through Informal Interaction in Low-Tech Clusters UNU-MERIT Dissertation Series, № 82

\section{Flavia Pereira de Carvalho}

What we talk about when we talk about Brazilian Multinationals: An Investigation on Brazilian FDI, Economic Structure, Innovation and the Relationship between them UNU-MERIT Dissertation Series, № 81

\section{Jun Hou}

Complementarity in Innovation and Development: A Cross-country

Comparison

UNU-MERIT Dissertation Series, № 80

\section{Rufin Baghana}

Impacts of Government Incentives to $R \mathcal{E D}$, Innovation and Productivity: A Microeconometric Analysis of the Québec Case

UNU-MERIT Dissertation Series, № 79

\section{Lilia I. Stubrin}

High-Tech Activities in Emerging Countries: A Network perspective on the Argentinean Biotech Activity UNU-MERIT/MGSoG Dissertation Series, № 78

\section{Kristine Farla}

Empirical Studies on Institutions, Policies and Economic Development MGSoG Dissertation Series, № 38 
Marina Petrovic

Social Assistance and Activation in the Pursuit of Happiness: Shedding New

Light on Old Policy Solutions to Social Exclusion

MGSoG Dissertation Series, № 37

\section{Laura Torvinen}

Assessing Governance Assessments:

The Case of Mozambique: Governance

Assessments in the Context of Aid

Effectiveness Discourse

MGSoG Dissertation Series, № 36

\section{Biniam Egu Bedasso}

Institutional Change in the Long

Shadow of Elite: Essays on

Institutions, Human Capital and

Ethnicity in Developing Countries

MGSoG Dissertation Series, № 35

Sepideh Yousefzadeh FaalDeghati

Childhoods Embargoed: Constructing

and Reconstructing Multidimensional

Child Poverty in Iran 1984-2009

MGSoG Dissertation Series, № 34

\section{Robert Bauchmüller}

Investing in Early Childhood Care and

Education: The Impact of Quality on

Inequality

MGSoG Dissertation Series, № 33

\section{Martin Rehm}

Unified Yet Separated: Empirical

Study on the Impact of Hierarchical

Positions within Communities of

Learning

MGSoG Dissertation Series, № 32
2012

\author{
Abdul Waheed \\ Innovation Determinants and \\ Innovation as a Determinant: Evidence \\ from Developing Countries \\ UNU-MERIT Dissertation Series, \\ № 77
}

\section{Bilal Mirza}

Energy Poverty and Rural Energy

Markets in Pakistan

UNU-MERIT Dissertation Series, № 76

\section{Benjamin Engelstätter}

Enterprise Software and Video Games:

An Empirical Analysis

UNU-MERIT Dissertation Series, № 75

\section{Fulvia Farinelli}

Natural Resources, Innovation and Export Growth: The Wine Industry in Chili and Argentina

UNU-MERIT Dissertation Series

\section{Rodolfo Lauterbach}

Innovation in Manufacturing: From

Product Variety and Labor

Productivity Growth to Economic

Development in Chile

UNU-MERIT Dissertation Series

\section{Kirsten Wiebe}

Quantitative Assessment of

Sustainable Development and Growth in Sub-Saharan Africa

UNU-MERIT Dissertation Series, № 74 


\section{Julio Miguel Rosa}

Organizational Strategies, Firms'

Performance and Spatial Spillovers:

The Canadian Case in Research and

Development.

UNU-MERITDissertation Series, №

73

Johannes Wilhelmus Marie Boels

Joseph Schumpeter, Honderd Jaar

Economische Ontwikkeling: Een

Historisch-theoretische Beschouwing.

UNU-MERIT Dissertation Series

\section{Dorcas Mbuvi}

Utility Reforms and Performance of the

Urban Water Sector in Africa

MGSoG Dissertation Series, № 31

\section{Lina Salanauskaite}

Distributional Impacts of Public

Policies: Essays in Ex-Ante and Ex-

Post Evaluation

MGSoG Dissertation Series, № 30

\section{Esther Schüring}

To Condition or not - is that the Question?

An Analysis of the Effectiveness of ExAnte and Ex-Post Conditionality in

Social Cash Transfer Programs

MGSoG Dissertation Series, № 29

\section{Joe Abah}

Strong Organisations in Weak States:

Atypical Public Sector Performance in

Dysfunctional Environments

MGSoG Dissertation Series, № 28

\section{Zina Samih Nimeh}

Social Citizenship Rights: Inequality

and Exclusion

MGSoG Dissertation Series, № 27

2011

\section{Daniel Vertesy}

Interrupted Innovation: Emerging

Economies in the Structure of the

Global Aerospace Industry

UNU-MERIT Dissertation Series, № 72

\section{Tina Saebi}

Successfully Managing Alliance

Portfolios: AnAlliance Capability View UNU-MERIT Dissertation Series, № 71

\section{Nora Engel}

Tuberculosis in India: A Case of

Innovation and Control

UNU-MERIT/MGSoG Dissertation

Series, № 70

\section{Evans Mupela}

Connectivity and growth in Sub-

Saharan Africa: The Role of

Communication Satellites

UNU-MERIT Dissertation Series, № 69

\section{Nantawan Kwanjai}

Cross Cultural Intelligence amid

Intricate Cultural Webs: A Tale of the

UnDutchables in the Land of 1002

Smiles

UNU-MERIT Dissertation Series, № 68 


\section{Lina Sonne}

Innovation in Finance to Finance

Innovation: Supporting Pro-poor

Entrepreneur-based Innovation

UNU-MERIT Dissertation Series, № 67

\section{Lenka Eisenhamerová}

Legitimacy of 'Humanitarian Military

Intervention'

MGSoG Dissertation Series, № 26

\section{Sonila Tomini}

Informal Payments for Health Care

Services in Albania

MGSoG Dissertation Series, № 25

\section{Jinjing Li}

Dynamic Microsimulation in Public

Policy Evaluation

MGSoG Dissertation Series, № 24

\section{Aziz Atamanov}

Rural Nonfarm Employment and

International Migration as

Alternatives to Agricultural

Employment: The Case of Kyrgyzstan

MGSoG Dissertation Series, № 23

\section{Frieda Vandeninden}

Poverty Alleviation: Aid and Social

Pensions

MGSoG Dissertation Series, № 22

\section{Juliana Nyasha Tirivayi}

The Welfare Effects of Integrating

AIDS Treatment with Food Transfers:

Evidence from Zambia

MGSoG Dissertation Series, № 21

\section{AgnieskaEwa Sowa}

Who's Left Behind? Social Dimensions

of Health Transition and Utilization of

Medical Care in Poland

MGSoG Dissertation Series, № 20

\section{Emmanaouil Sfakianakis}

The Role of Private Actors in the

Provision of Public Goods with

Applications to Infrastructure and

Financial Stability

MGSoG Dissertation Series, № 19

\section{Siu Hing Lo}

White Collars Green Sleeves: An Inter-

organizational Comparison of

Determinants of Energy-Related

Behaviors among Office Workers

MGSoG Dissertation Series, № 18

\section{Treena Wu}

Constraints to Human Capital

Investment in Developing Countries:

Using the Asian Financial Crisis in

Indonesia as a Natural Experiment

MGSoG Dissertation Series, № 17

\section{Henry Espinoza Peña}

Impact Evaluation of a Job-Training

Programme for Disadvantaged Youths:

The Case of Projoven

MGSoG Dissertation Series, № 16 


\section{Asel Doranova}

Fernando Santiago

Human Resources Management

Practices and Learning for Innovation

in Developing Countries:

Pharmaceutical Firms in Mexico

UNU-MERIT Dissertation Series,

№ 66

Technology Transfer and Learning

under the Kyoto Regime: Exploring the

Technological Impact of CDM Projects

in Developing Countries

UNU-MERIT Dissertation Series, № 61

\section{Florian Tomini}

Between Family and Friend:

Zakaria Babutsidze

Essays on Economies with

Heterogeneous Interacting Consumers

UNU-MERIT Dissertation Series, № 65

Understanding the Interdependency of Private Transfers

MGSoG Dissertation Series, № 15

\section{Michał Polalowski}

The Institutional Transformation of

\section{Bertha Vallejo}

Learning and Innovation Under

Changing Market Conditions: The

Auto Parts Industry in Mexico

UNU-MERIT Dissertation Series,

№ 64

Social Policy in East Central Europe:

Poland and Hungary in Comparative

and Historical Perspective

MGSoG Dissertation Series, № 14

\section{Maha Ahmed}

Defining, Measuring and Addressing

Vulnerability: The Case of Post

Conflict Environments

MGSoG Dissertation Series, № 13

\section{Pascal Beckers}

Local Space and Economic Success:

The Role of Spatial Segregation of

Migrants in the Netherlands

MGSoG Dissertation Series, № 12

Multinational Subsidiary Evolution:

Corporate Change in New EU Member States

UNU-MERIT Dissertation Series, № 62

\section{Victor Cebotari}

Conflicting Demands in Ethnically

Diverse Societies: Ethno political

Contention and Identity Values in

Europe

MGSoG Dissertation Series, № 11 
Dennis Gyllensporre

Competing and Complementary

Perspectives on the EU as a Crisis

Management Actor:

An Examination of the Common

Security and Defence Policy through

the Lenses of Idealism and Realism

MGSoG Dissertation Series, № 10

Judit Vall Castello

Business Cycle and Policy Effects on

Labour Market Transitions of Older

and Disabled Workers in Spain

MGSoG Dissertation Series, № 9

\section{Keetie Roelen}

False Positives or Hidden Dimensions:

The Definition and Measurement of

Child Poverty

MGSoG Dissertation Series, № 8

Denisa Maria Sologon

Earning Dynamics in Europe

MGSoG Dissertation Series, № 7

\section{Melissa Siegel}

Money and Mobility: Migration and

Remittances

MGSoG Dissertation Series, № 6

Jessica S. Hagen-Zanker

Modest Expectations: Causes and

Effects of Migration on Migrant

Households inSource Countries

MGSoG Dissertation Series, № 5
2009

\begin{abstract}
Alexis Habiyaremye
From Primary Commodity Dependence to Diversification and Growth:

Absorptive Capacity and Technological Catch Up in Botswana and Mauritius.

UNU-MERIT Dissertation Series, № 60
\end{abstract}

\section{Yoseph Getachew}

The Role of Public Capital in Economic Development

UNU-MERIT Dissertation Series, № 59

\section{Sandra Leitner}

Embodied Technological Change and

Patterns of Investment in Austrian

Manufacturing

UNU-MERIT Dissertation Series, № 58

\section{SemihAkçomak}

The Impact of Social Capital on Economic and Social Outcomes UNU-MERIT Dissertation Series, № 57

\section{Abraham Garcia}

The Role of Demand in Technical

Change

UNU-MERIT Dissertation Series, № 56

\section{Saurabh Arora}

Coherence in Socio-technical Systems:

A Network Perspective on the Innovation Process

UNU-MERIT Dissertation Series, № 55 


\section{Mirtha R. Muniz Castillo}

Human Development and Autonomy

in Project Aid: Experiences from four

bilateral projects in Nicaragua and $E l$

Salvador

MGSoG Dissertation Series, № 4

\section{Christiane Arndt}

Governance Indicators

MGSoG Dissertation Series, № 3

\section{Britta Augsburg}

Microfinance: Greater Good or Lesser

Evil?

MGSoG Dissertation Series, № 2

2008

\section{Rutger Daems}

Medicines for the Developing World

UNU-MERIT Dissertation Series, № 54

\section{Johannes Hanel}

Assessing Induced Technology:

Sombart's Understanding of Technical

Change in the History of Economics

UNU-MERIT Dissertation Series,

№ 53

\section{Rifka Weehuizen}

Mental Capital: the Economic

Significance of Mental Health

UNU-MERIT Dissertation Series,

№ 52

\section{Danielle Cloodt}

The Relationship between RED

Partnership Formation, Social

Embeddedness and Innovative

Performance

UNU-MERIT Dissertation Series, № 51

\section{Sabine Fuss}

Sustainable Energy Development under Uncertainty

UNU-MERIT Dissertation Series, № 50

\section{Geranda Notten}

Measuring and Managing Poverty

Risks

MGSoG Dissertation Series, № 1

2007

\section{Tobias Kronenberg}

Reconciling Environmental

Conservation with Economic

Prosperity: The Feasibility of Double

Dividends in the Short and Long Run UNU-MERIT Dissertation Series, № 49

\section{Viktoria Kravtsova}

Assessing the Impact of Foreign Direct Investment in Transition Economies UNU-MERIT Dissertation Series, № 48 


\section{Suhail Sultan}

The Competitive Advantage of Small and Medium Sized Enterprises: The Case of Jordan's Natural Stone Industry

UNU-MERIT Dissertation Series, № 47

2006

\section{Bulat Sanditov}

Essays on Social Learning and Imitation

UNU-MERIT Dissertation Series, № 46

\section{Mamata Parhi}

Dynamics of New Technology Diffusion: A Study of the Indian Automotive Industry

UNU-MERIT Dissertation Series, № 45

\section{Andreas Reinstaller}

Social Structures and the Innovation Process: Their Role in the Demand of Firms and Consumers

UNU-MERIT Dissertation Series, № 44

\section{Rose Kiggundu}

Innovation systems and Development:

The Journey of a Beleaguered Nile

Perch Fishery in Uganda

UNU-MERIT Dissertation Series, № 43

\section{Thomas Pogue}

The Evolution of Research

Collaboration in South African Gold Mining: 1886-1933

UNU-MERIT Dissertation Series, № 42

\section{Geoffrey Gachino}

Foreign Direct Investment, Spillovers and Innovation: The Case of Kenyan Manufacturing Industry

UNU-MERIT Dissertation Series, № 41

\section{ÖnderNomaler}

Technological Change, International Trade and Growth: An Evolutionary, Multi-Agents-Based Modeling

Approach

UNU-MERIT Dissertation Series, № 40

2005

Samia Satti Osman Mohamed-

Nour

Change and Skill Development in the Arab Gulf Countries

UNU-MERIT Dissertation Series, № 39

\section{Elad Harison}

Intellectual Property Rights:

Economics and Policy Analysis

UNU-MERIT Dissertation Series, № 38 


\section{Daniel Dalohoun}

The Relationship between RED

Partnership Formation, Social

Embeddedness and Innovative

Performance: a Multi-level Approach of Social Embeddedness

UNU-MERIT Dissertation Series, № 37

\section{Müge Ozman}

Networks, Organizations and

Knowledge

UNU-MERIT Dissertation Series,

№ 36

\section{Bas Straathof}

Product Variety and Economic

Growth: The Counteracting Effects of

Scale and Idiosyncrasy

UNU-MERIT Dissertation Series,

№ 35

\section{Wilfred Schoenmakers}

Knowledge Flows between

Multinational Companies: A Patent

Data Analysis

UNU-MERIT Dissertation Series, № 34

\section{Myriam Cloodt}

Mergers and Acquisitions ( $M$ and As) in High-Tech Industries: Measuring the Post-M and A Innovative

Performance of Companies

UNU-MERIT Dissertation Series, № 33
2004

\section{Paola Criscuolo}

RED Internationalisation and

Knowledge Transfer: Impact on MNEs and their Home Countries

UNU-MERIT Dissertation Series, № 32

\section{Maarten Verkerk}

Trust and Power on the Shop Floor UNU-MERIT Dissertation Series, № 31

\section{Gottfried Leibbrandt}

Adoption, Harmonization and Succession of Network Technologies across Countries

UNU-MERIT Dissertation Series, № 30

\section{Mark Sanders}

Skill Biased Technical change: Its Origins, the Interaction with the Labour Market and Policy Implications UNU-MERIT Dissertation Series, № 29

2003

Nadine Roijakkers

Inter-firm Cooperation in High-tech Industries: a Study of RED

Partnerships in Pharmaceutical Biotechnology

UNU-MERIT Dissertation Series, № 28 
Viki Sonntag

Speed, Scale and Sustainability

UNU-MERIT Dissertation Series, № 27

\section{Masaru Yarime}

From End-of-Pipe Technology to Clean Technology

UNU-MERIT Dissertation Series, № 26

\section{StéphaneMalo}

The Combinatorial Chemistry Revolution: Sustaining a Superior Performance Position through Technological Learning UNU-MERIT Dissertation Series, № 25

2002

\section{Annelies Hogenbirk}

Determinants of Inward Foreign

Direct Investment: the Case of the

Netherlands

UNU-MERIT Dissertation Series, № 24

\section{Bastiaan Johan terWeel}

The Computerization of the Labour Market

UNU-MERIT Dissertation Series
2001

\section{John Adeoti}

Technology Investment in Pollution Control in Sub-Saharan Africa: The Case of the Nigerian Manufacturing Industry

UNU-MERIT Dissertation Series, № 23

\section{Edward Huizenga}

Innovation Management: How

Frontrunners Stay Ahead: An

Empirical Study on Key Success

Factors in the ICT sector

UNU-MERIT Dissertation Series, № 22

\section{0}

\section{Machiel van Dijk}

Technological Change and the Dynamics of Industries: Theoretical Issues and Empirical evidence from Dutch Manufacturing UNU-MERIT Dissertation Series, № 21

\section{9}

\section{Jan Cobbenhagen}

Managing Innovation at the Company Level: A Study on Non-Sector-Specific Success Factors UNU-MERIT Dissertation Series, № 20 
Marjolein Caniëls

Regional Growth Differentials: The

Impact of Locally Bounded Knowledge

Spillovers

UNU-MERIT Dissertation Series, № 19

1998

\begin{abstract}
Aldo Geuna
Resource Allocation and Knowledge production: Studies in the Economics of University Research UNU-MERIT Dissertation Series, № 18
\end{abstract}

1996

\section{Reinoud Joosten}

Dynamics, Equilibria, and Values UNU-MERIT Dissertation Series, № 17

\section{Hugo Kruiniger}

Investment, $R \mathcal{E} D$, and the Financing

Decisions of the Firm

UNU-MERIT Dissertation Series, № 16

\section{5}

\section{Hans van Meijl}

Endogenous Technological Change:

The Case of Information Technology,

Theoretical Considerations and

Empirical Results

UNU-MERIT Dissertation Series, № 15

\section{René Kemp}

Environmental Policy and Technical

Change: A Comparison of the

Technological Impact of Policy

Instruments

UNU-MERIT Dissertation Series, № 14

\section{Rohini Acharya}

The Impact of New Technologies on

Economic Growth and Trade: A Case

Study of Biotechnology

UNU-MERIT Dissertation Series, № 13

\section{Geert Duysters}

The Evolution of Complex Industrial Systems: The Dynamics of Major IT Sectors

UNU-MERIT Dissertation Series, № 12

\section{MarjanGroen}

Technology, Work and Organisation: A Study of the Nursing Process in Intensive Care Units

UNU-MERIT Dissertation Series, № 11

\section{4}

\section{Huub Meijers}

On the Diffusion of Technologies in a Vintage Framework: Theoretical Considerations and Empirical Results UNU-MERIT Dissertation Series, № 10 
Theon van Dijk

The Limits of Patent Protection: Essays on the Economics of Intellectual

Property Rights

UNU-MERIT Dissertation Series, № 9

\section{Hans Voordijk}

Naar Integrale Logistiek in

Bedrijfsketens: Ontwikkelingen in de Bouw

UNU-MERIT Dissertation Series, № 8

1993

\section{Paul Diederen}

Technological Progress in Enterprises and Diffusion of Innovation:

Theoretical Reflections and Empirical

Evidence

UNU-MERIT Dissertation Series, № 7

\section{Ben Dankbaar}

Economic Crisis and Institutional Change: The Crisis of Fordism from the Perspective of the Automobile Industry UNU-MERIT Dissertation Series, № 6

\section{Hanno Roberts}

Accountability and Responsibility: The Influence of Organisation Design on Management Accounting UNU-MERIT Dissertation Series, № 5
1992

\section{Bart Verspagen}

Uneven Growth between

Interdependent Economies: An

Evolutionary View on Technology

Gaps, Trade and Growth

UNU-MERIT Dissertation Series,

№ 4

\section{Sjoerd Romme}

A Self-organization Perspective on

Strategy Formation

UNU-MERIT Dissertation Series, № 3

\section{9}

John Spangenberg

Economies of Scale, and Atmosphere in Research Organisations

UNU-MERIT Dissertation Series, № 2

1988

\section{John Hagedoorn}

Evolutionary and Heterodox Innovation Analysis: A Study of Industrial and Technological Development in Process Control and Information Technology UNU-MERIT Dissertation Series, № 1 


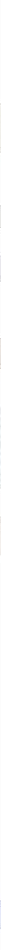

